\title{
Propagation and Morphology of Premixed Flames in Obstructed Channels under Atmospheric and Supercritical Conditions
}

\author{
ABDULAFEEZ AKINOLA ADEBIYI \\ West Virginia University, abadebiyi@mix.wvu.edu
}

Follow this and additional works at: https://researchrepository.wvu.edu/etd

Part of the Energy Systems Commons, and the Heat Transfer, Combustion Commons

\author{
Recommended Citation \\ ADEBIYI, ABDULAFEEZ AKINOLA, "Propagation and Morphology of Premixed Flames in Obstructed \\ Channels under Atmospheric and Supercritical Conditions" (2019). Graduate Theses, Dissertations, and \\ Problem Reports. 3761. \\ https://researchrepository.wvu.edu/etd/3761
}

This Dissertation is protected by copyright and/or related rights. It has been brought to you by the The Research Repository @ WVU with permission from the rights-holder(s). You are free to use this Dissertation in any way that is permitted by the copyright and related rights legislation that applies to your use. For other uses you must obtain permission from the rights-holder(s) directly, unless additional rights are indicated by a Creative Commons license in the record and/ or on the work itself. This Dissertation has been accepted for inclusion in WVU Graduate Theses, Dissertations, and Problem Reports collection by an authorized administrator of The Research Repository @ WVU.

For more information, please contact researchrepository@mail.wvu.edu. 
2019

Propagation and Morphology of Premixed Flames in Obstructed Channels under Atmospheric and Supercritical Conditions

ABDULAFEEZ AKINOLAADEBIYI

Follow this and additional works at: https://researchrepository.wvu.edu/etd

Part of the Energy Systems Commons, and the Heat Transfer, Combustion Commons 


\title{
Propagation and Morphology of Premixed Flames in Obstructed Channels under Atmospheric and Supercritical Conditions
}

\author{
Abdulafeez Akinola Adebiyi \\ Dissertation submitted \\ to the Statler College of Engineering and Mineral Resources \\ at West Virginia University
}

in partial fulfilment of the requirements for the degree of

\author{
Doctor of Philosophy in \\ Mechanical Engineering \\ V'yacheslav Akkerman, Ph.D., Chair \\ Arvind Thiruvengadam Padmavathy, Ph.D. \\ Cosmin Dumitrescu, Ph.D . \\ Terence Musho, Ph.D. \\ Damir Valiev, Ph.D.
}

Department of Mechanical and Aerospace Engineering

Morgantown, West Virginia

2019

Keywords: combustion, premixed flame propagation, premixed flame morphology, deflagration to detonation transition, obstructed channels, computational simulation, Bychkov mechanism, Peng Robinson equation of state, supercritical flames.

Copyright 2019 Abdulafeez Akinola Adebiyi 


\title{
ABSTRACT \\ Propagation and Morphology of Premixed Flames in Obstructed Channels under Atmospheric and Supercritical Conditions
}

\begin{abstract}
Abdulafeez Akinola Adebiyi
The understanding of the morphology and propagation of premixed flames in channels is vital for the design and development of efficient propulsion systems requiring high heat release, such as pulse-detonation engines, and for resolving accidental fire in industrial processes. In this dissertation, extensive computational simulations of premixed flames in channels with rectangular and cylindrical cross sections, with closely packed obstacles are carried out. The goal is to provide insights into flame propagation under various conditions, which would be invaluable to the development of next-generation energy and propulsion systems as well as fire safety of industrial processes.

Factors that may intensify or attenuate premixed flame propagation include the geometry, the thermochemical properties based on the thermal expansion ratio and on the fuel mixture Lewis number (the ratio of the thermal to mass diffusivities), and the operating conditions. In semi-open channels, a flame may accelerate while propagating from the closed end of the channel to the open end. Much stronger flame acceleration occurs when a channel is packed with a tooth-brush-like array of obstacles. This is a so-called Bychkov mechanism, according to which, flame acceleration is devoted to delayed burning in the pockets formed by adjacent obstacles.

The first part of this dissertation is focused on premixed flames under atmospheric conditions. The starting point is to investigate the effect of wall shear stress and the Lewis number on flame acceleration in semi-open obstructed channels. It is found that the effects of wall shear stress play a significant role only when the obstacle spacing exceeds the radius (half-width) of the channel. However, the impact of the Lewis number, Le, is significant. Specifically, flame acceleration weakens for $L e>1$, inherent to fuel-rich hydrogen or fuel-lean propane burning. In contrast, the $L e<1$ flames, corresponding to the fuel-lean hydrogen or fuel-rich propane mixtures, are prone to extra strong folding of the flame front and thereby accelerate faster. The later effect can be devoted to the onset of the diffusional-thermal combustion instability. A strong interplay is observed to
\end{abstract}


exist between the Lewis number and the blockage ratio (the ratio of the obstacle height to the halfwidth of the channel).

While semi-open channels replicate the geometry of many propulsion systems, premixed flames in channels with both ends open may represent the scenario of an accidental fire in a coal mine tunnel or other numerous industrial and laboratory conduits. In such channels with closelyplaced obstacles, flame oscillations, or acceleration, or their sequences have been observed. While the oscillations are generally inherent to relatively narrow channels, in wider ones, the accelerative trend eventually dominates over the oscillations. Both the accelerating and the oscillatory trends depend strongly on the thermochemical properties of the mixtures and geometrical factors.

The next part of this dissertation is focused on supercritical combustion. Since, supercritical fluids are known to have unique thermophysical properties that can potentially be harnessed in developing highly efficient energy systems with near-zero emission levels. For this reason, the key characteristics of supercritical $\mathrm{CO}_{2}$-diluted, oxy-methane premixed flames at 300 bar and $800 \mathrm{~K}$ are investigated. It is observed that an increase in the $\mathrm{CO}_{2}$-dilution rate makes the flame thicker and reduces the unstretched laminar flame speed as compared to the non-diluted cases. Starting with a planar flame, the analysis is subsequently extended to the corrugated flames due to non-slip walls and obstacles, with the dynamics and morphology of such a corrugated flame front identified. While a corrugated flame generally spreads faster than a planar one having the same thermalchemical characteristics, the corrugated flame velocity reduces with the dilution rate. In the limit of high $\mathrm{CO}_{2}$-dilution, the Bychkov mechanism is observed to promote acceleration. Overall, the Bychkov theory predicts well the propagation velocity of a supercritical flame as long as the dilution is moderate. 
To my father and mother for raising me and inspiring me to greatness in life. 


\section{ACKNOWLEDGEMENTS}

All praise be to God Almighty for sparing my life and guiding me through my PhD journey.

My special thanks go to my advisor, Dr. V'yacheslav Akkerman for giving me the wonderful opportunity to work in his research group and for his incredible support through my $\mathrm{PhD}$. His expert guidance equipped me with top-notch leadership skills as well as adept ability to understand and decipher complex research problems.

I am grateful to the NSF for providing funding to conduct the research on flame propagation at atmospheric conditions as well as Dr. Kemenov and the US DOE for providing funding support which aided the supercritical flame propagation research. Special thanks go to one of my advisory committee members, Dr. Valiev, for providing me with the computational platform utilized for my research work. My profound gratitude also goes to the members of my dissertation committee, Dr Thiruvengadam, Dr. Dumitrescu, and Dr. Musho for the constant advice and constructive criticism. I also wish to thank my colleagues, especially Orlando Ugarte, Sinan Demir and Jad Sadek for guiding me through the early stages of my research. And likewise, I am thankful to my research colleagues who have contributed directly to this work, Olatunde Abidakun, Elizabeth Ridgeway, Amanda Cathreno, Gbolahan Idowu and all my colleagues at the analytical and computational combustion group.

I also wish to thank my friends, Dr Tajudeen Iwalewa, Lateef Kareem and Ilias Oyenuga, for their support through this journey. Finally, my most profound gratitude goes to my parents, Mr. and Mrs. Adebiyi for the relentless prayers and support, as well as my siblings, Tope, Shade and Damola Adebiyi, who were constant sources of encouragement at difficult times; I am sure I have made you all proud. 


\section{Table of Contents}

List of Tables ............................................................................................................ vii

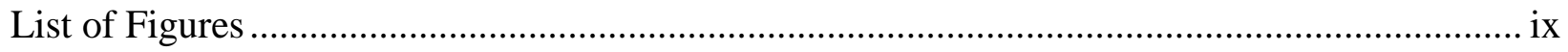

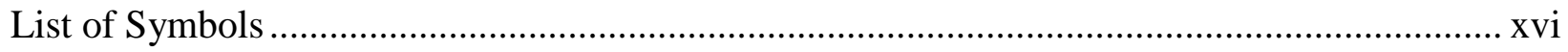

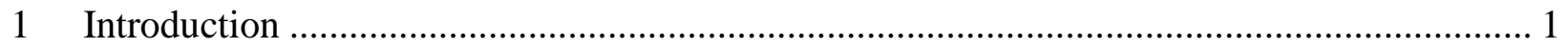

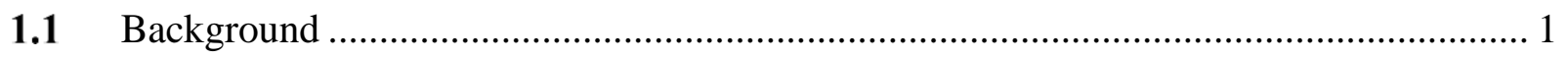

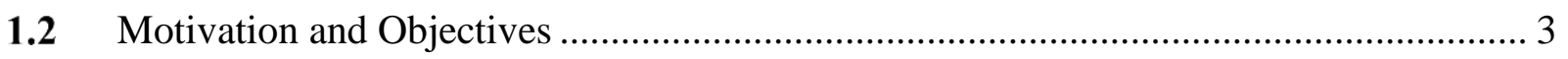

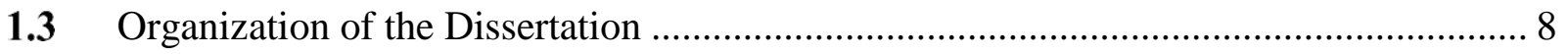

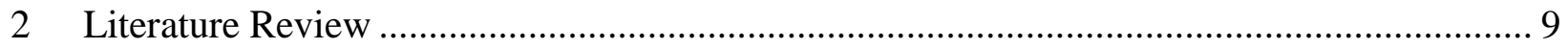

2.1 Premixed Flames in Semi-Open Channels ............................................................. 9

2.2 Premixed Flames in Channels with Both Ends Open ............................................ 15

2.3 Premixed Flames with $\mathrm{CO}_{2}$-dilutions............................................................. 16

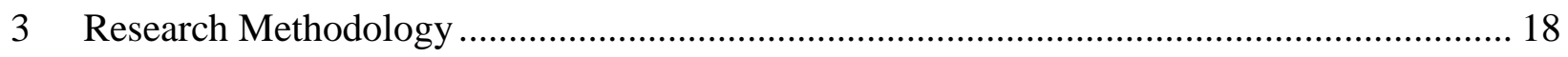

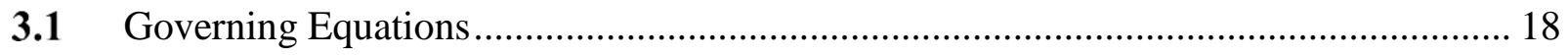

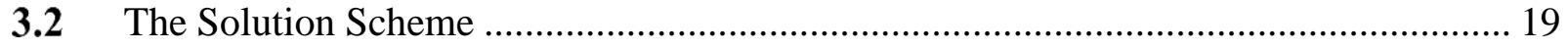

3.2.1 Ideal Gas Model - Original Solver ................................................................. 19

3.2.2 Real Gas Model - Modified Solver..................................................................... 19

3.3 Grid/Mesh Generation ...................................................................................... 22

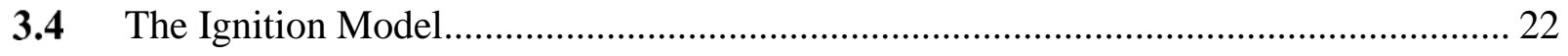

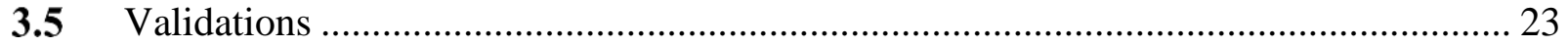

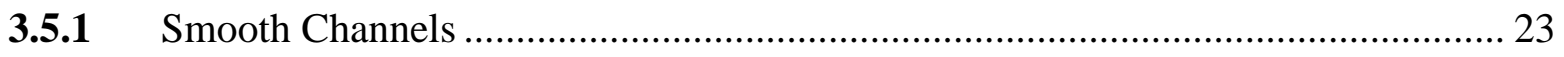

3.5.2 Obstructed Channel..................................................................................... 25

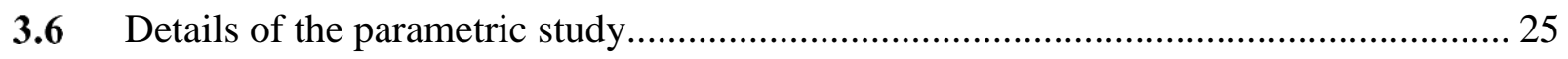

4 Flame Acceleration in Semi-Open Cylindrical Channels............................................... 26

4.1 Effects of the Wall Surface on Flame Acceleration: $R / 4 \leq \Delta Z \leq R / 2 \ldots \ldots \ldots \ldots \ldots \ldots \ldots . . . . .26$

4.2 Effects of the Wall Surface Friction on Flame Acceleration: $R / 2 \leq \Delta Z \leq R \ldots \ldots \ldots \ldots .28$

4.3 Effects of the Wall Surface of Flame Acceleration: $\Delta Z=2 R$............................... 31

5 Flame Acceleration in Semi-open Obstructed Channels ............................................... 34

5.1 Morphology of Premixed Flame in Semi-open Channels ........................................ 34

5.2 Quantitative Effect of the Lewis number on Flame Acceleration.............................. 35

5.3 Effect of Blockage Ratio on Flame Acceleration..................................................... 36 
5.4 Effect of Channel Width on Flame Acceleration .......................................................... 37

6 Flame Oscillation in Obstructed Channels with Both Ends Open......................................... 40

6.1 Morphology of Oscillating Premixed Flame................................................................ 40

6.2 Impacts of Blockage Ratio on an Oscillating Premixed Flame ………………….......... 41

6.3 Impacts of Obstacle Spacing on an Oscillating Premixed Flame ………..................... 42

6.4 Impacts of the Thermal Expansion Ratio on an Oscillating Premixed Flame ................ 43

6.5 Unified Characteristics of an Oscillating Premixed Flame.............................................. 44

7 Flame Acceleration in Obstructed Channels with Both Ends Open....................................... 46

7.1 Morphology of the Accelerating Premixed Flame ........................................................... 46

7.2 Characteristics of the Accelerating Premixed Flame ……………………………......... 47

7.3 Influence of the Geometrical and Thermochemical Factor............................................ 49

7.4 Validation of the Accelerating Flame Simulations by the Bychkov Theory .................. 51

7.5 Analysis of the Saturated Flame Regime …………….................................................... 53

7.6 Classification of the Flame Propagation Regimes ………………………………........... 54

8 Modeling of Supercritical $\mathrm{CO}_{2}$-Diluted Oxy-Methane Flames ............................................. 58

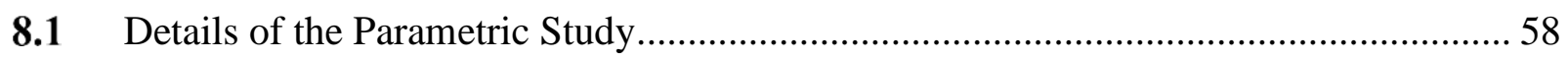

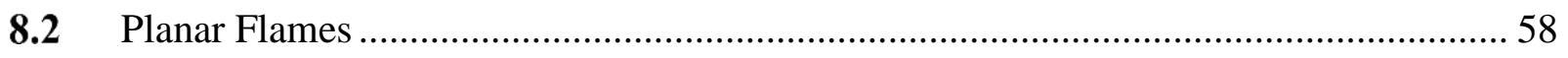

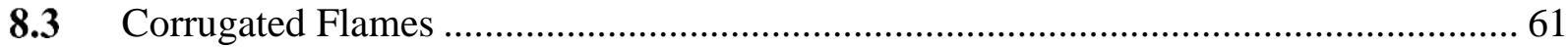

8.4 Estimation of the Markstein Length/Number............................................................. 70

9 Supercritical Flames in a Semi-Open Obstructed Channel ...................................................... 73

9.1 Flame Morphology and Propagation......................................................................... 73

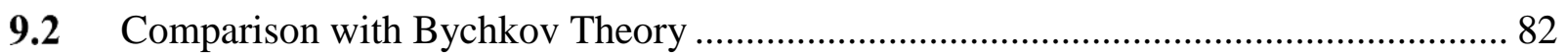

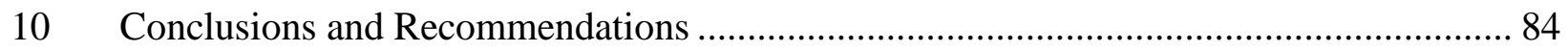

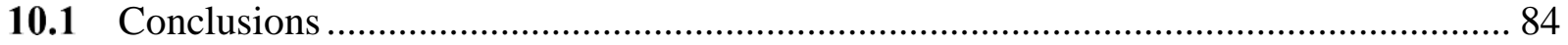

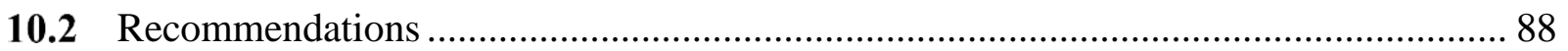

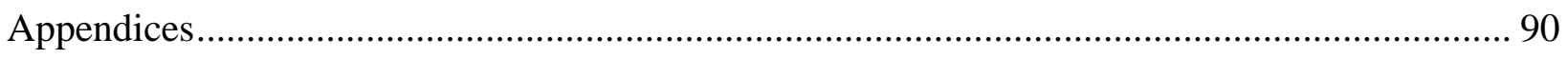

Appendix A: Fortran 77 Code of Peng Robinson Implementation ........................................... 90

Appendix B: Fortran 77 Code of Lucas Viscosity Model.......................................................... 93

Appendix C: C Code of Peng Robinson Implementation ........................................................ 95

Appendix D: C Code of Lucas Viscosity Model ..................................................................... 96

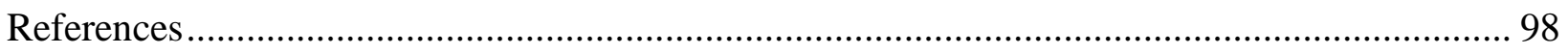




\section{List of Tables}

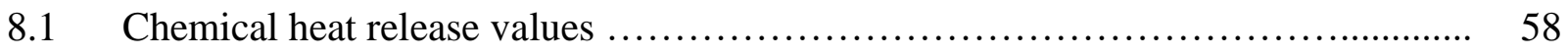

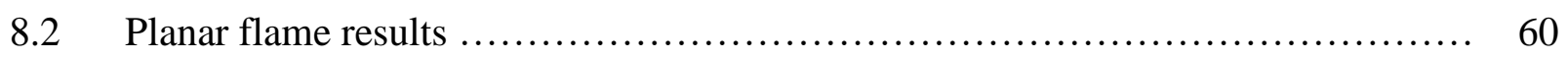




\section{List of Figures}

1.1 (a) Non-premixed flame configuration. (b) Characteristic distribution of the temperature and species fraction inside a planar non-premixed flame front.

1.2 (a) Premixed flame configuration. (b) Characteristic distribution of the temperature and density inside a planar flame front ......................................... 2

1.3 The specific energy densities of various energy systems ..................... 4

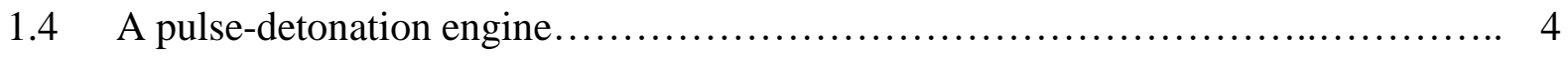

1.5 Schlieren video of flame propagation in channels with large obstacle spacing........... 5

1.6 An illustration of the Bychkov mechanism of flame acceleration in obstructed channels........................................................... 5

1.7 Schlieren video of flame propagation in channels with closely packed obstacles...... 6

1.8 A schematic of an obstructed channel with both ends open...................... 7

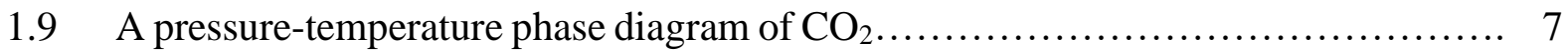

3.1 Viscosity at $T_{f}=800 \mathrm{~K}$ for a) $\beta=0 \%$ b) $P_{f}=300 \mathrm{bar} \ldots \ldots \ldots \ldots \ldots \ldots \ldots \ldots \ldots \ldots \ldots \ldots \ldots \ldots \ldots$

3.2 Schematic of the grid used in the numerical simulations...................... 22

3.3 Solution initialization based on a planar ignition............................ 23

3.4 Solution initialization based on a hemispherical ignition....................... 23

3.5 Experimental set-up of ethylene-oxygen mixture in a capillary tube............... 24

3.6 The flame tip evolution in a tube of radius $0.25 \mathrm{~mm} \ldots \ldots \ldots \ldots \ldots \ldots \ldots \ldots \ldots \ldots . .24$

3.7 Optical access to combustion channel with closely packed obstacles................. 24

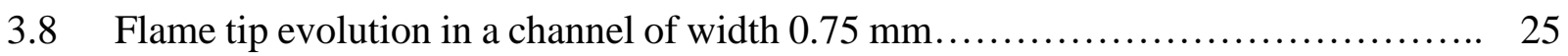

3.9 Resolution test: The scaled flame tip position versus the scaled time for $\alpha=1 / 3$, $L e=0.2$ and various square mesh sizes $\alpha=1 / 3, L e=0.2$ and various square

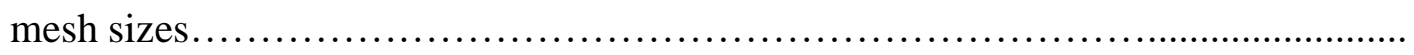

4.1 The scaled flame tip position $Z_{f} / R$ versus the scaled time $\tau=t S_{L} / R$ for $\alpha=2 / 3$, $\Delta Z / R=1 / 4$, and various $R=24 L_{f}$ and $36 L_{f}$ 
4.2 The scaled flame tip position $Z_{f} / R$ versus the scaled time $\tau=t S_{L} / R$ for $R=24 L_{f}$, $\Delta Z / R=1 / 4$, and various $\alpha=1 / 3,1 / 2$, and $2 / 3$

4.3 The scaled flame tip position $Z_{f} / R$ versus the scaled time $\tau=t S_{L} / R$ for $R=24 L_{f}$, $\alpha=2 / 3$ and various $\Delta Z / R=1 / 4$ and $1 / 2$.

4.4 The color temperature snapshots [in K] for burning in an obstructed tube with freeslip (a) and non-slip (b) walls for $\mathrm{R}=24 \mathrm{~L}_{\mathrm{f}}, \alpha=2 / 3, \Delta \mathrm{Z}=\mathrm{R} / 2$, and taken at the same time instant $\tau=\mathrm{tS}_{\mathrm{L}} / \mathrm{R}=0.15$ in both cases.

4.5 The scaled flame tip position $Z_{f} / R$ versus the scaled time $\tau=t S_{L} / R$ for $R=24 L_{f}$, $\alpha=2 / 3$ and various $\Delta Z / R=1 / 4,1 / 2$, and 1

4.6 The scaled flame tip position $Z_{f} / R$ versus the scaled time $\tau=t S_{L} / R$ for $R=12 L_{f}$, $\alpha=1 / 3$ and various $\Delta Z / R=1 / 4,1 / 2$, and 1

4.7 The scaled flame tip position $\mathrm{Z}_{\mathrm{f}} / \mathrm{R}$ vs the scaled time $\tau=\mathrm{tS}_{\mathrm{L}} / \mathrm{R}$ for $R=36 L_{f}$, $\alpha=1 / 3, \Delta Z / R=1$

4.8 The color temperature snapshots [in K] for burning in an obstructed tube with freeslip (a) and non-slip (b) walls for $R=36 L_{f}, \alpha=1 / 3, \Delta Z / R=1$ and taken at the same time instant $\tau=t S_{L} / R=0.225$ in both cases.

4.9 The color temperature snapshots [in K] for burning in an obstructed tube with freeslip (a) and non-slip (b) walls for $R=36 L_{f}, \alpha=1 / 2, \Delta Z / R=1$ and taken at the same time instant $\tau=t S_{L} / R=0.225$ in both cases.

4.10 The color temperature snapshots [in K] for burning in an obstructed tube with freeslip (a) and non-slip (b) walls for $R=36 L_{f}, \alpha=2 / 3, \Delta Z / R=1$ and taken at the same time instant $\tau=t S_{L} / R=0.225$ in both cases.

4.11 The scaled flame tip position $Z_{f} / R$ vs the scaled time $\tau=t S_{L} / R$ for $R=12 L_{f}$, $\alpha=1 / 3, \Delta Z / R=2$.

4.12 The scaled flame tip position $Z_{f} / R$ vs the scaled time $\tau=t S_{L} / R$ for $R=12 L_{f}$, $\alpha=2 / 3, \Delta Z / R=2$.

4.13 The color temperature snapshots [in K] for burning in an obstructed tube with free- 
slip (a) and non-slip (b) walls for $R=12 L_{f}, \alpha=1 / 3, \Delta Z / R=2$ and taken at the same time instant $\tau=t S_{L} / R=0.36$ in both cases.......................... 32

4.14 The color $\theta$-vorticity snapshots [in $\mathrm{sec}^{-1}$ ] for burning in an obstructed tube with slip (a) and non-slip (b) walls for $R=12 L_{f}, \alpha=1 / 3, \Delta Z / R=2 \tau=t S_{L} / R=0.36 \ldots . .33$

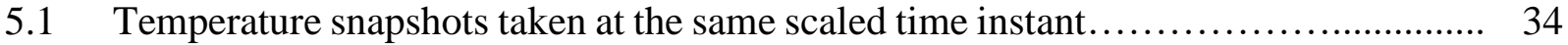

5.2 The scaled flame tip velocity $U_{\text {tip }} / S_{L}$ vs the scaled time $\tau$ for $R / L_{f}=24$ and $\alpha=$ $1 / 3$ (a), $1 / 2$ (b), $2 / 3$ (c). Figure (d) shows the scaled flame tip velocity $U_{\text {tip }} / S_{L}$ at $\tau=0.075$ versus Le for different values of $\alpha=1 / 3,1 / 2,2 / 3 \ldots \ldots \ldots \ldots \ldots \ldots \ldots$

5.3 The scaled flame tip velocity $U_{\text {tip }} / S_{L}$ versus the scaled time $\tau$ for $R / L_{f}=24$ and various $L e=0.2(\mathrm{a}), 0.5(\mathrm{~b}), 1.0(\mathrm{c}), 2.0(\mathrm{~d})$

5.4 The scaled flame tip velocity $U_{\text {tip }} / S_{L}$ versus the scaled time $\tau$ for $\alpha=2 / 3$ and various $L e=0.2(\mathrm{a}), 0.5(\mathrm{~b}), 1.0(\mathrm{c}), 2.0(\mathrm{~d})$

5.5 The exponential acceleration rate $\sigma$ versus the Lewis number $L e$ for $R / L_{f}=24$ (a) 36 (b) 48 (c), with $\alpha=1 / 3,1 / 2$, and $2 / 3$ in each figure.

5.6 The scaled flame tip position $\sigma Z_{f} / \Theta R$ versus the scaled time $\sigma t S_{L} / R$ for each various $L e=0.2$ (square markers), 1.0 (triangle markers) and 2.0 (without marker), with three different $\alpha=1 / 3$ (solid), 1/2 (dashed) and 2/3 (dotted) for given Le.....

6.1 Consecutive color temperature snapshots and the flow streamlines (white) for the propagation of a flame with the thermal expansion ratio $\Theta=8$ in a channel with the obstacle spacing $\Delta z=R / 4$ and various blockage ratios: $\alpha=1 / 3$ (a), $\alpha=1 / 2$ (b), and $\alpha=2 / 3$ (c). (d) and (e) are the zoomed versions of the snapshots (ii) and (iv) for $\alpha=1 / 2$

6.2 The scaled burning rate $U_{w} / S_{L}$ versus the scaled time $S_{L} t / R$ for the thermal expansion ratio $\Theta=8$, obstacle spacing $\Delta z=R / 4$, and various blockage ratios: $\alpha=1 / 3$ (a), $\alpha=1 / 2$ (b), and $\alpha=2 / 3$ (c).

6.3 The scaled burning rate $U_{w} / S_{L}$ versus the scaled time $S_{L} t / R$ for the thermal expansion ratio $\Theta=8$, the blockage ratio $\alpha=1 / 3$ and various obstacle spacing 
$\Delta z=R / 4$ (a), $\Delta z=R / 2$ (b) and $\Delta z=R(\mathrm{c})$

6.4 The scaled burning rate $U_{w} / S_{L}$ versus the scaled time $S_{L} t / R$ for the blockage ratio $\alpha=1 / 3$, obstacle spacing $\Delta z=R / 4$ and various thermal expansion ratios $\Theta=5$

(a), $\Theta=8(\mathrm{~b}), \Theta=10$ (c).

6.5 The scaled burning rate $U_{w} / S_{L}$ versus the scaled time $S_{L} t / R$ for the blockage ratio $\alpha=1 / 2$, obstacle spacing $\Delta z=\mathrm{R} / 4$ and various thermal expansion $\operatorname{ratios} \Theta=5$

(a), $\Theta=8(b), \Theta=10$ (c)

6.6 The scaled burning rate $U_{w} / S_{L}$ versus the scaled time $S_{L} t / R$ for the blockage ratio $\alpha=2 / 3$, obstacle spacing $\Delta z=R / 4$ and various thermal expansion ratios $\Theta=5$

(a), $\Theta=8$ (b), $\Theta=10$ (c).

6.7 The scaled oscillation period $\tau_{p} S_{L} / R$ (a), the average burning rate during an oscillation $\left\langle U_{w}\right\rangle / S_{L}$ (b), and the scaled oscillation amplitude $\Delta U / S_{L}$ (c) versus the thermal expansion ratio $\Theta$ for the obstacles spacing $\Delta z=R / 4$ and various blockage

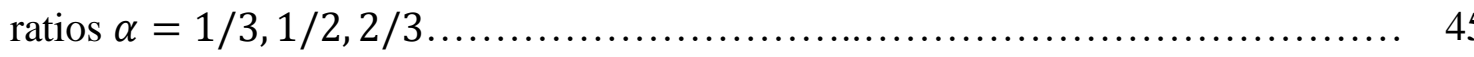

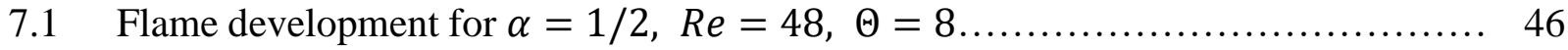

7.2 Various flame propagation trends for $\alpha=1 / 2, \Theta=8$ : a) $Z_{f} / R$ b) $U_{t i p} / S_{L} \ldots \ldots \ldots \ldots . . . \quad 48$

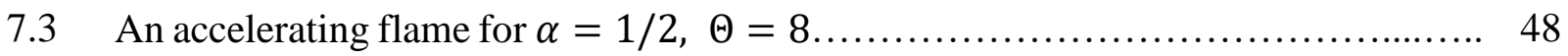

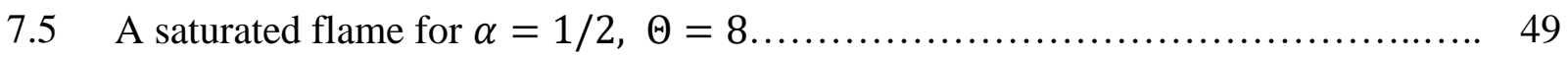

7.6 Flame propagation for $\Theta=8, \mathrm{R}=24 L_{f}:$ a) $Z_{f} / R$ vs $t \cdot S_{L} / R$ b) $A_{f} / A$ vs $t \cdot S_{L} / R \ldots \quad 49$

7.7 Flame propagation for $\Theta=8, R=36 L_{f}:$ a) $Z_{f} / R$ vs $t \cdot S_{L} / R$ b) $A_{f} / A$ vs $t \cdot S_{L} / R . . \quad 50$

7.8 Flame propagation for $\Theta=8, R=48 L_{f}:$ a) $\mathrm{Z}_{\mathrm{f}} / \mathrm{R}$ vs $t \cdot S_{L} / R$ b) $\mathrm{A}_{\mathrm{f}} / \mathrm{A}$ vs $t \cdot S_{L} / R \ldots 50$

7.9 The scaled burning velocity $U_{w} / S_{L}$ versus the scaled time $t S_{L} / R$ for $\alpha=2 / 3$,

$\Theta=8$.

7.10 The scaled burning velocity $U_{w} / S_{L}$ versus the scaled time $t S_{L} / R$ for $\alpha=2 / 3$,

$R=48 L_{f}$

7.11 The scaled flame tip position versus the scaled time for $R=24 L_{f}:$ a) $\alpha=1 / 2$

b) $\alpha=2 / 3$. 
7.12 The scaled flame tip position versus the scaled time for $R=36 L_{f}$ : a) $\alpha=1 / 2$

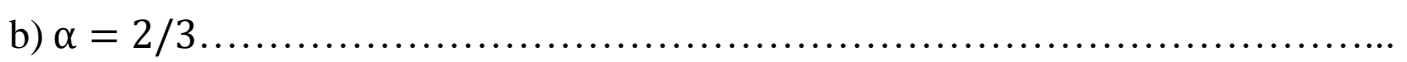

7.13 The scaled flame tip position versus the scaled time for $R=48 L_{f}$ : a) $\alpha=1 / 2$

b) $\alpha=2 / 3$.

7.14 The scaled flame tip velocity versus its scaled position: a) $\Theta=8$ b) $\Theta=10 \ldots \ldots . . .54$

7.15 Machine learning model representation.................................... 55

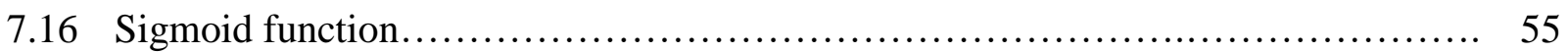

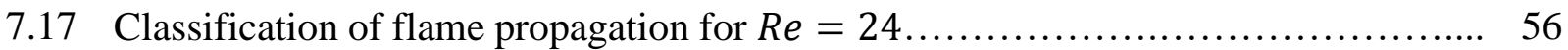

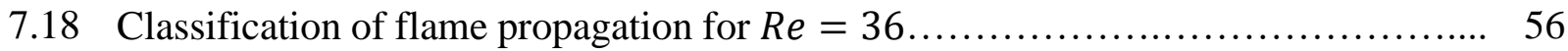

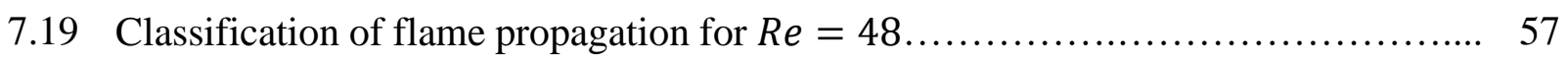

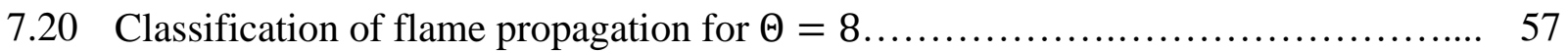

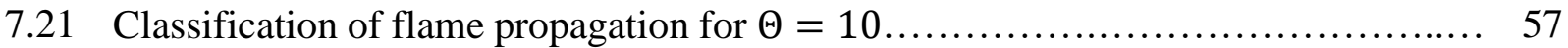

8.1 Planar flame speeds vs time at $P_{f}=1$ bar a) comparing of IG with PR b) grid resolution check................................................... 58

8.2 Planar flame speeds for $T_{f}=800 \mathrm{~K}, P_{f}=300 \mathrm{bar}$ (a) speed vs time (b) speed vs dilution rates.

8.3 The density (a) and temperature (b) profiles at $T_{f}=800 \mathrm{~K}, P_{f}=300$ bar......... 61

8.4 Profiles at $T_{f}=800 \mathrm{~K}, P_{f}=300 \mathrm{bar}$ (a) fuel mass fraction (b) flame thickness $\beta \ldots \quad 61$

8.5 Flame snapshots in a $20 \mu \mathrm{m}$ channel (a) $\beta=0$ (b) $\beta=40 \%$, (c) $\beta=60 \%$, (d) $\beta=80 \%$

8.6 Velocity profile just ahead of the flame in a $20 \mu \mathrm{m}$ channel (a) $x$-component (b) scaled $\mathrm{x}$-component

8.7 Shear stress ahead of the flame front: $\beta=0$ (a) $20 \%$ (b) $40 \%$ (c) $60 \%$ (d) $80 \%$ (e) at wall $\mathrm{y}=0$ (f)

8.8 Profiles for $\beta=0$ (a) the corrugated flame velocity; (b) the scaled flame surface area.

8.9 Profiles for $\beta=20 \%$ : (a) the corrugated flame velocity; (b) the scaled flame 


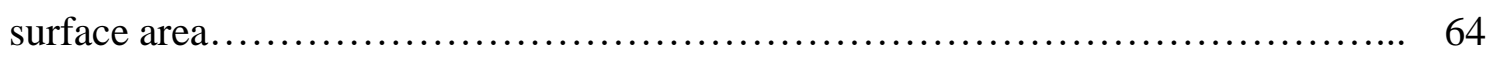

8.10 Profiles for $\beta=40 \%$ (a) the corrugated flame velocity; (b) the scaled flame

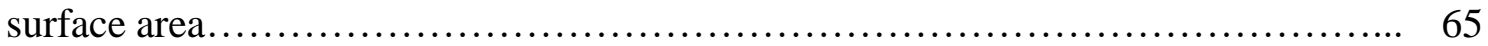

8.11 Profiles for $\beta=60 \%$ (a) the corrugated flame velocity; (b) the scaled flame surface

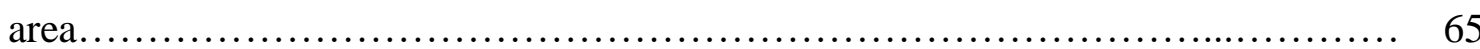

8.12 Profiles for $\beta=80 \%$ (a) the corrugated flame velocity; (b) the scaled flame

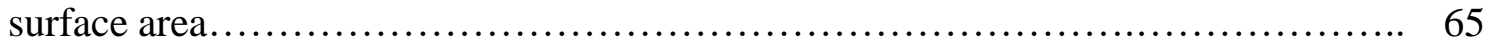

8.13 Profiles for $D=5 \mu \mathrm{m}$ (a) the corrugated flame velocity; (b) the scaled flame surface area.

8.14 Profiles for $D=10 \mu \mathrm{m}$ (a) the corrugated flame velocity (b) the scaled flame surface area

8.15 Profiles for $D=20 \mu \mathrm{m}$ (a) the corrugated flame velocity; (b) the scaled flame surface area.......

8.16 Profiles for $D=40 \mu \mathrm{m}$ (a) the corrugated flame velocity; (b) the scaled flame surface area.

8.17 Flame shape variation for $D=20 \mu \mathrm{m}$ (a) $\beta=0 \%$ (b) $\beta=40 \%$ (c) $\beta=60 \%$ (d) $\beta=80 \%$.

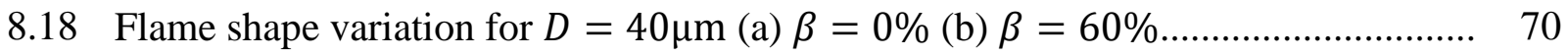

8.19 Markstein length predictions (a) $\beta=0 \%$ (b) $\beta=40 \%$ (c) $\beta=60 \%$ (d) $\beta=80 \%$. 71

8.20 Dilution effect on (a) Markstein length (b) Markstein number........................ 72

9.1 Flame snapshots for $\beta=0 \%, \alpha=1 / 3, D=12 \mu \mathrm{m}$ at times a) $\mathrm{t}=0.35 \mu \mathrm{s}$ ) $t=$ $0.7 \mu \mathrm{s}$.

9.2 Flame snapshots for $\beta=0 \%, \alpha=1 / 2, \mathrm{D}=12 \mu \mathrm{m}$ at times a) $t=0.25 \mu \mathrm{s}$ ) $t=$ $0.5 \mu \mathrm{s}$

9.3 Flame snapshots for $\beta=0 \%, \alpha=2 / 3, \mathrm{D}=12 \mu \mathrm{m}$ at times a) $\mathrm{t}=0.35 \mu \mathrm{s}$ b) $t=$ $0.7 \mu \mathrm{s}$.

9.4 Flame tip velocity for $\beta=0 \%$, a) $\alpha=1 / 2$ b) $\alpha=2 / 3$ c) $D=12 \mu \mathrm{m}$. 75

9.5 Flame snapshots for $\beta=40 \%, \alpha=1 / 3, D=12 \mu \mathrm{m}$. 76 


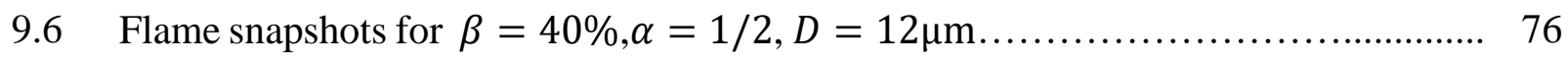

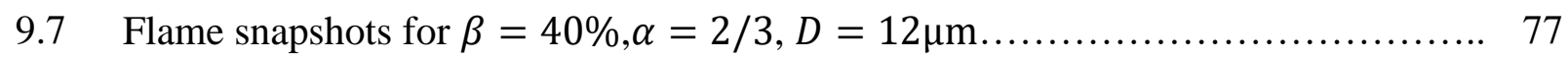

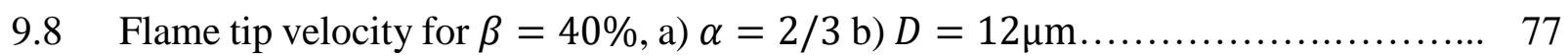

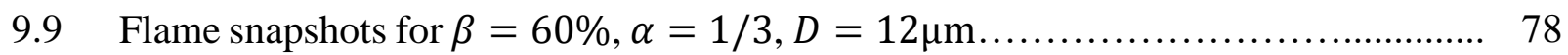

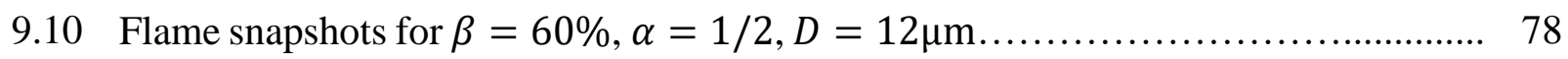

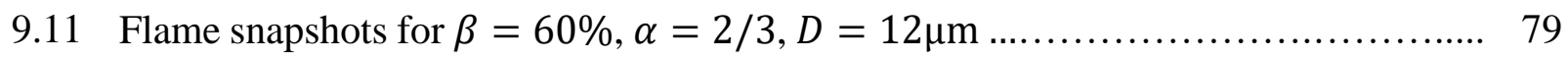

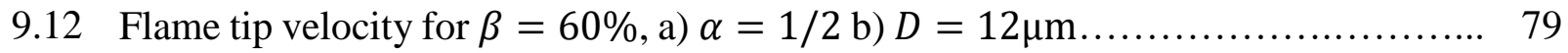

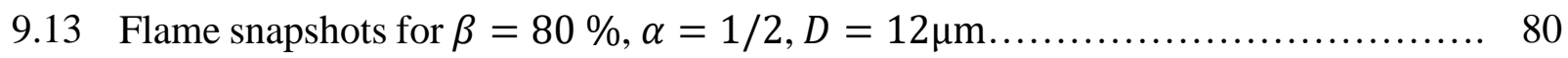

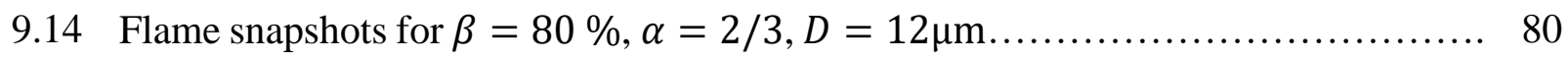

9.15 The scaled flame length vs time for $D=6 \mu \mathrm{m}$ and $\beta=80 \%$ a) $\alpha=1 / 2$,

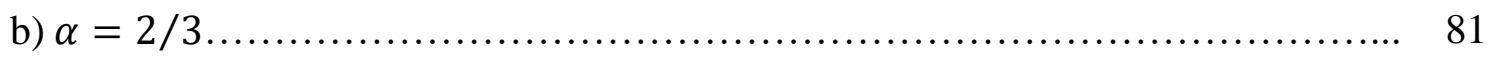

9.16 The scaled flame tip length vs time for $D=24 \mu \mathrm{m}, \alpha=2 / 3$ and various $\beta \ldots \ldots \ldots . .81$

9.17 a) The scaled flame tip velocity vs time for $D=24 \mu \mathrm{m}, \alpha=2 / 3$

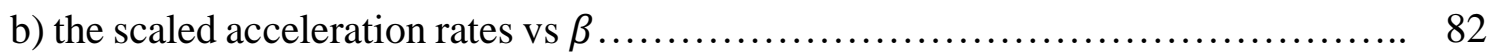

9.18 The scaled flame tip velocity versus the scaled flame tip position for $D=24 \mu \mathrm{m}$

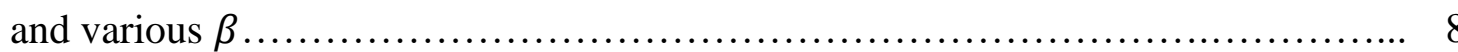




\section{List of Symbols}

\section{English Letters}

\begin{tabular}{|c|c|}
\hline$C_{V}$ & Specific heat capacity at constant volume \\
\hline$C_{P}$ & Specific heat capacity at constant pressure \\
\hline$c_{0}$ & Speed of the sound in the unburned fuel mixture \\
\hline$D$ & Channel width/height, tube diameter \\
\hline$E_{a}$ & Activation energy \\
\hline$F_{P_{m}}$ & Lucas viscosity model correction factor \\
\hline$K$ & Flame stretch rate \\
\hline$k_{i, j}$ & Binary interaction parameters between species $i, j$ \\
\hline$L$ & Channel/tube length \\
\hline$L_{b}$ & Markstein length \\
\hline Le & Lewis number \\
\hline$L_{f}$ & Flame thickness \\
\hline$M$ & Molecular weight \\
\hline$M a$ & Mach number \\
\hline$M k$ & Markstein number \\
\hline $\bar{n}$ & Normal vector \\
\hline$P$ & Pressure \\
\hline$P_{f}$ & Initial pressure \\
\hline $\operatorname{Pr}$ & Prandtl number \\
\hline
\end{tabular}




\begin{tabular}{|c|c|}
\hline$P_{r_{m}}$ & Reduced pressure of the mixture \\
\hline$P_{c m}$ & Critical pressure of mixture \\
\hline$Q$ & Specific energy released in a chemical reaction \\
\hline$r$ & Radial position \\
\hline$r_{f}$ & Radial position of the flame as function of time \\
\hline$R$ & Tube radius/channel half-width \\
\hline$R e$ & Flame Reynolds number \\
\hline$R_{u}$ & Universal gas constant \\
\hline$S c$ & Schmidt number \\
\hline$S_{L}$ & Laminar flame speed \\
\hline$S_{b}^{0}$ & Unstretched laminar flame speed with respect to the burnt matter \\
\hline$S_{b}$ & Stretched radial flame velocity \\
\hline$T$ & Temperature \\
\hline$T_{f}$ & Initial temperature of fuel mixture \\
\hline$T_{u}$ & Cold (unburned) fuel mixture temperature \\
\hline$T_{b}$ & Burned matter temperature \\
\hline$T_{a d}$ & Adiabatic flame temperature \\
\hline$T_{c m}$ & Critical temperature of mixture \\
\hline$t$ & Time (in seconds) \\
\hline$T_{r_{m}}$ & Reduced temperature of the mixture \\
\hline$u$ & Velocity component \\
\hline
\end{tabular}




$\begin{array}{ll}U_{t i p} & \text { Flame tip velocity } \\ U_{W} & \text { Corrugated flame velocity } \\ U_{1} & \text { Flow velocity at burned extreme end } \\ U_{2} & \text { Flow velocity at cold extreme end } \\ X_{i} & \text { Species mole fraction } \\ Y & \text { Mass fraction of the fuel mixture } \\ Z_{1} & \text { Pressure correction parameter } \\ Z_{2} & \text { Temperature correction parameter } \\ Z_{f} & \text { Flame tip position } \\ Z & \text { Axial direction }\end{array}$




\section{Greek Letters}

\begin{tabular}{ll}
$\alpha$ & Blockage ratio \\
$\gamma$ & Specific heat ratio \\
$\Theta$ & Dilution rate \\
$\Delta Z$ & Thermal expansion ratio \\
$\tau$ & Obstacle spacing \\
$\tau_{\mathrm{P}}$ & Scaled time \\
$\sigma$ & Reaction time constant \\
$\rho$ & Acceleration rate \\
$\eta$ & Density \\
$\varepsilon$ & Dynamic viscosity \\
$\xi$ & Kinematic viscosity \\
& Energy release per unit volume \\
& Molar specific volume \\
\hline &
\end{tabular}




\section{Introduction}

\subsection{Background}

Combustion is one of the most essential processes for the sustenance of human lives. Its application ranges from heat and power generation to transportation as well as other areas of life. Modern combustion studies are typically focused on energy production and fire safety. Indeed, about 85\% of the world energy production still originates from combustion [1], and despite the growing trends in renewable energy technology, combustion is expected to power our industries for several decades to come, due to its convenience, high-energy density, and relatively low cost [2]. Overall, the role of combustion in energy production, its potential for catastrophic disasters and its hazardous pollution effects, causing severe environmental and safety concerns, makes the research in the field of combustion very important. Combustion is an exothermic chemical reaction of a fuel and oxidizer.

In terms of mixing between the fuel and oxidizer before a reaction starts, combustion process can be classified as premixed and non-premixed combustion. When the fuel and oxidizer are perfectly mixed before the ignition, the burning process is called premixed combustion. In contrast, in a non-premixed system, the fuel and oxidizer remain separated prior to their involvement in the reaction zone; in other words, ignition occurs at the mixing layer between the fuel and the oxidizer. Premixed combustion is of primary practical importance to gasoline engines, stationary gas turbines, detonation engines and fire explosions. Here, combustion occurs by means of propagation of a front separating unburned fuel-oxidizer mixture from the combustion products. On the other hand, wild/forest fires, candle light or diesel engines are one of the most used applications of nonpremixed combustion. Moreover, understanding the nature of flame propagation is crucial for controlling and prevention of sudden fires and explosions. 

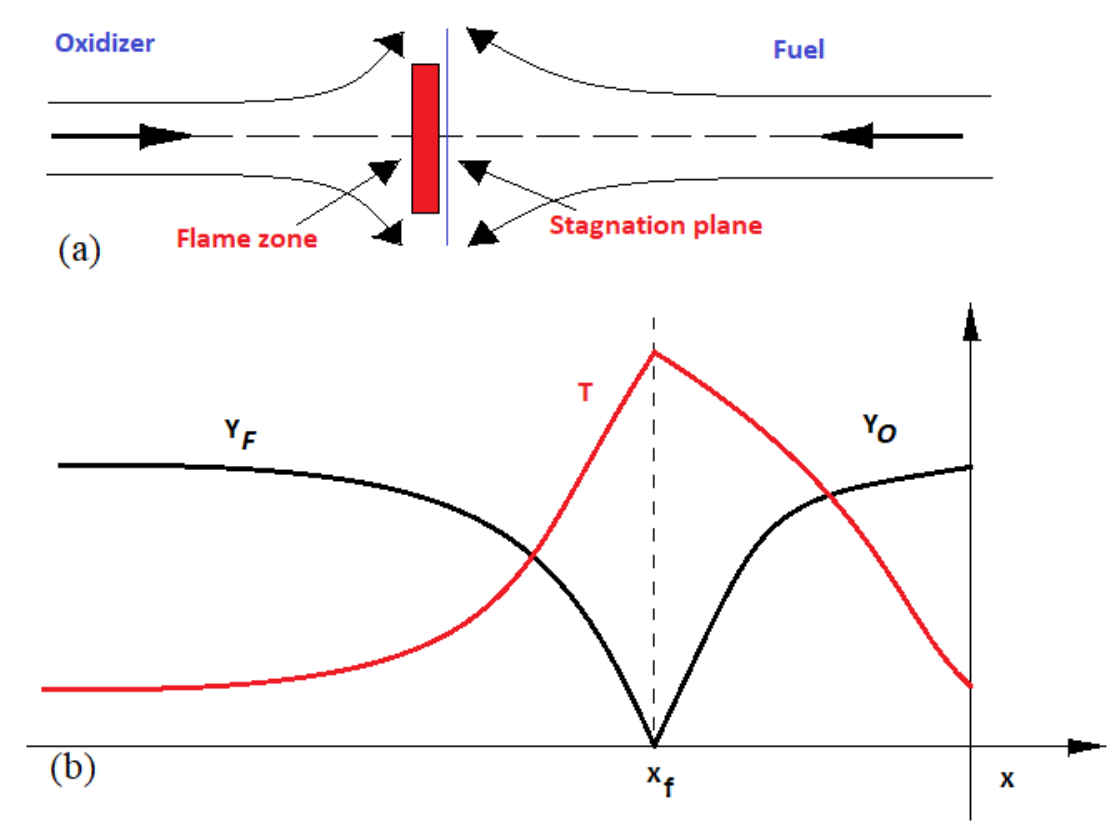

Figure 1.1: (a) Non-premixed flame configuration. (b) Characteristic distribution of the temperature and species fraction inside a planar non-premixed flame front.

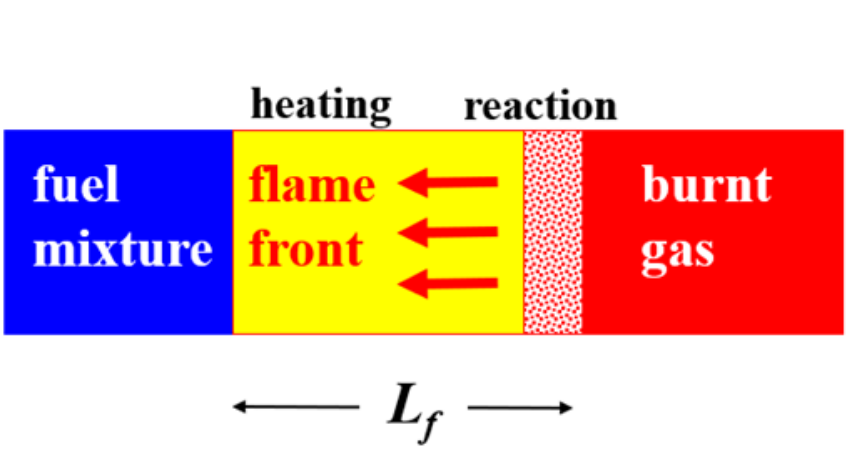

(a)

(b)

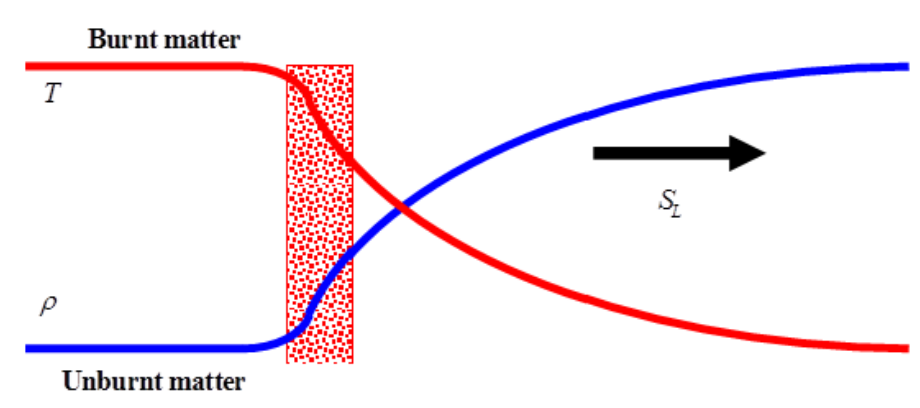

Figure 1.2: (a) Premixed flame configuration. (b) Characteristic distribution of the temperature and density inside a planar flame front.

Non-premixed combustion occurs more frequently in nature. As seen in Fig. 1.1, a reaction sheet (flame zone) separates the fuel from the oxidizer with the products of the reaction flowing in both directions. Combustion is sustained by simultaneous diffusion of the fuel and oxidizer at 
the flame zone interface. In premixed combustion, shown in Fig. 1.2, the reaction zone is preceded by heat conduction (preheat zone). The length of the preheat zone defines the flame thickness and the laminar flame speed is the characteristic speed at which a planar flame travels relative to the unburned gas. The major quantities describing premixed flames are defined below.

$$
\begin{aligned}
& \text { Equivalence ratio: } \phi=\frac{(F / O)}{(F / O)_{s t}} \\
& \text { Thermal expansion ratio: } \Theta=\frac{\rho_{f}}{\rho_{b}} \\
& \text { Lewis number: } L e=\frac{D_{t h}}{D_{m}} \\
& \text { Flame thickness: } \mathrm{L}_{\mathrm{f}}=\frac{D_{t h}}{S_{L}} \\
& \text { Flame Reynolds number: } R e=\frac{R S_{L}}{\mu}=\frac{R}{L_{f}}
\end{aligned}
$$

\subsection{Motivation and Objectives}

Among all energy conversion systems, the fossil-fuel-based systems have the highest energy density [3], see Fig. 1.3, which makes them attractive for power generation and propulsion systems. As a result, it is essential to fully understand the characteristics of propagating flames in various geometries.

Fundamentally, premixed flame propagation is studied in free space, confinements and channels. However, flame propagation in channels may replicate the processes in most propulsion systems and accidental fire scenario in industrial processes. Premixed flames propagating in channels may experience various behaviors such as oscillation [4], acceleration [5] or a sequence

of these trends [6]. In channels with unobstructed walls, these behaviors are caused mainly by the wall boundary conditions. Specifically, a flame may accelerate in a channel with non-slip, adiabatic walls and one end open, when propagating from the closed to the open end, because the entire new volume generated in the burning process is pushed towards the single exit in such a "semi-open" channel. In the pioneering work of Shelkin [7], devoted to premixed flame propagation in tubes, an expansion of the burned gas, pushes the fuel mixture, which becomes nonuniform due to the non-slip walls. With the addition of turbulence, the flame front corrugates, 
burning strengthens and flame acceleration (FA) occurs which may lead to detonation. While FA and the deflagration-to-detonation transition (DDT) are usually considered as unwanted in process industries, on the other hand, they can be constructively employed in improving various advanced combustion technologies such as pulse-detonation engines shown in Fig. 1.4 [9, 10].

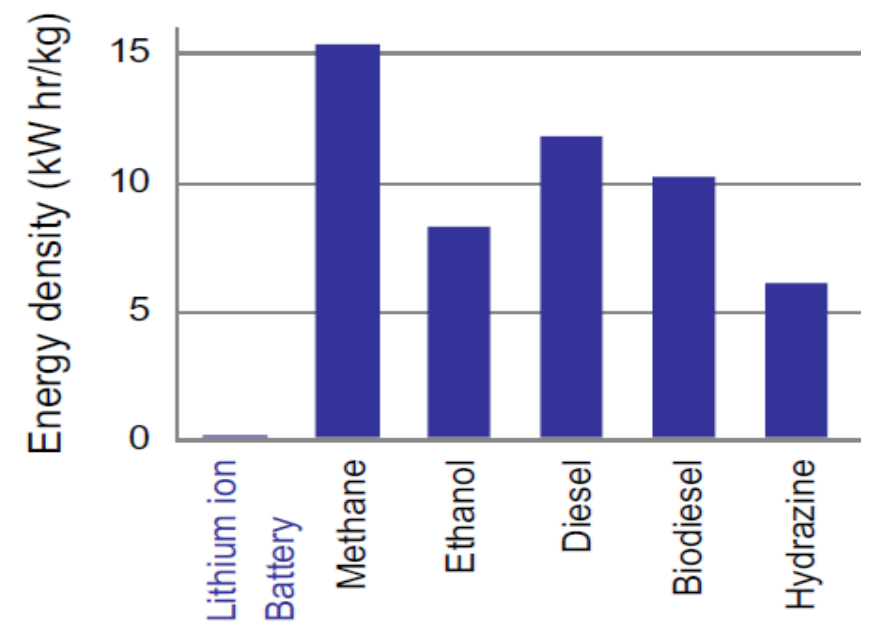

Figure 1.3: The specific energy densities of various energy systems [3].

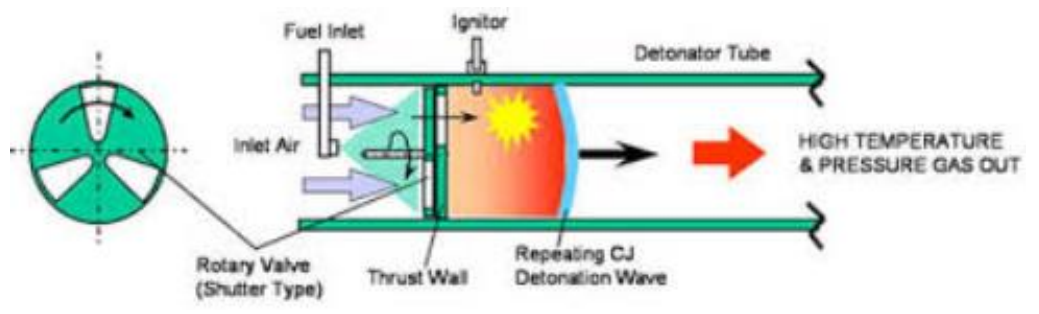

Figure 1.4: A pulse-detonation engine [9].

Among geometries associated with FA and DDT, obstructed channels provide the fastest regime of burning [10]. When obstacles are sparely placed in a channel, as shown in Fig. 1.5, flame propagation through such an obstructed channel is oftentimes associated with turbulence, shocks or hydraulic resistance [12-15]. A conceptually different mechanism of flame propagation was identified by Bychkov et al. [16-19]. This mechanism is laminar, scale-invariant (Reynoldsindependent), shockless, and it provides ultrafast FA in semi-open channels equipped with a toothbrush-like (comb-shaped) array of obstacles caused by delayed burning in pockets formed by adjacent obstacles. Eventually, the burned gas moves towards the unobstructed part of the channel from the opposing pockets, with gas flow velocity changing predominantly to the axial direction, resulting in a jet flow which drives the leading flame front. More new pockets are formed and 
ignited due to propagation of the flame tip, and accumulation of burnt gases coming out of these pockets promote faster propagation. The continuous feedback between the tip velocity and ignition in the new pockets promotes FA that may result in a detonation onset. This mechanism is referred to as the Bychkov mechanism, as illustrated in Fig. 1.6, and it is characterized by the blockage ratio $\alpha$, which is the ratio of the obstacle height $(\mathrm{H})$ to the channel half-width $(\mathrm{R})$, and the obstacles spacing $\Delta Z$. The experimental results [19], shown in Fig. 1.7, provide validation to this mechanism.

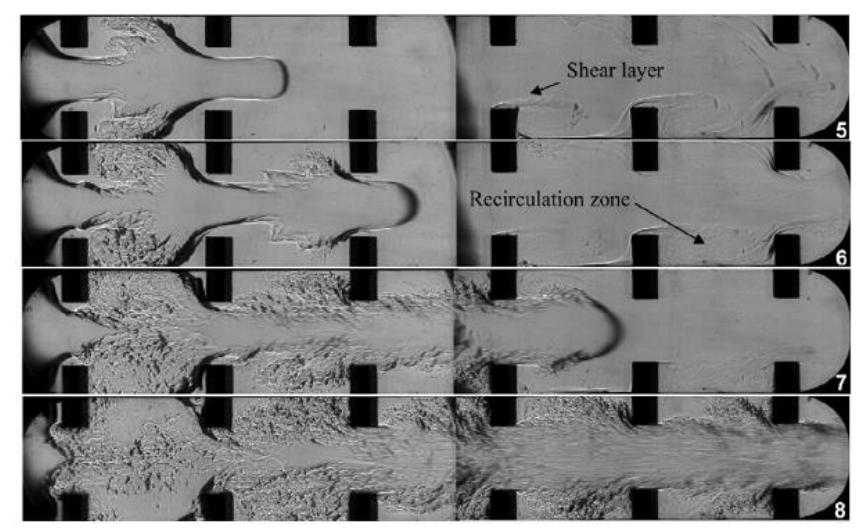

Figure 1.5: Schlieren video of flame propagation in channels with large obstacle spacing [20].
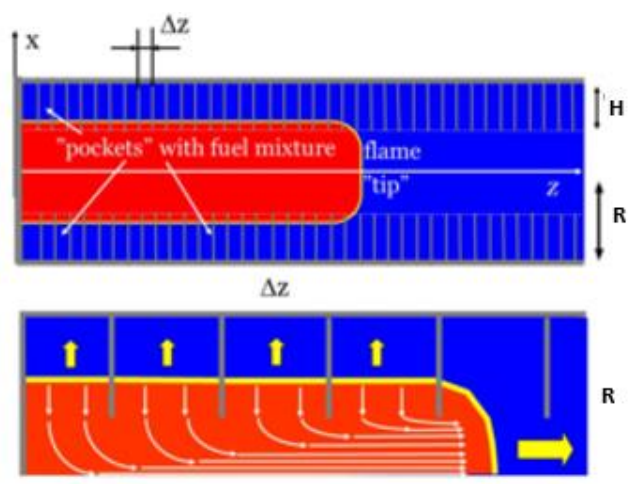

Figure 1.6: An illustration of the Bychkov mechanism of flame acceleration in obstructed channels [16-19]. 


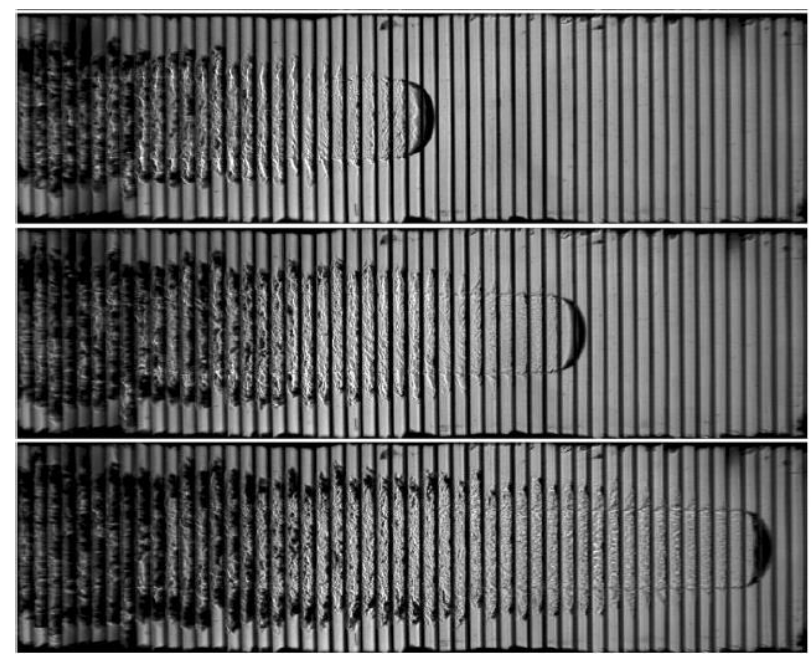

Figure 1.7: Schlieren video of flame propagation in channels with closely packed obstacles [19].

To fully utilize the Bychkov mechanism in practical systems, it is important to scrutinize this mechanism extensively. The theory and modelling of the mechanism [15] employed a set of simplifying assumptions, one of which is the free-slip walls. However, slip walls are relevant for a non-continuum flow, while the physical boundary conditions are non-slip as far as the continuum assumptions are valid. Indeed, it was shown that both wall shear stress [21] and thermal (cold and hot) wall conditions [5] play enormous roles in unobstructed channels. Will it be the case for obstructed ones? This poses the first question that is answered in this dissertation. While Ugarte et $a l$. [18] have shown a minor effect of the isothermal walls as compared to the adiabatic ones, in obstructed channels; in the present work, both slip and non-slip surfaces are compared to understand if wall shear stress plays important role in the Bychkov mechanism. The second assumption follows the conventional approach of equidiffusive combustion, when the Lewis number $L e=1$. Consequently, the Bychkov model needs to be validated in terms of its applicability to the practical reality, that is varying $L e$ conditions [22].

Studies of flame propagation, reported in the literature, often consider semi-open channels. However, flame propagation also occurs in channels with both ends opened. In this case the newlygenerated gas volume is distributed between the upstream and downstream flow (towards both ends), which eventually moderates FA. Akkerman et al. [4] identified oscillations in such a configuration. The next question addressed in this dissertation is what happens to a premixed flame in an obstructed channel with both ends open such as that illustrated in Fig. 1.8. 


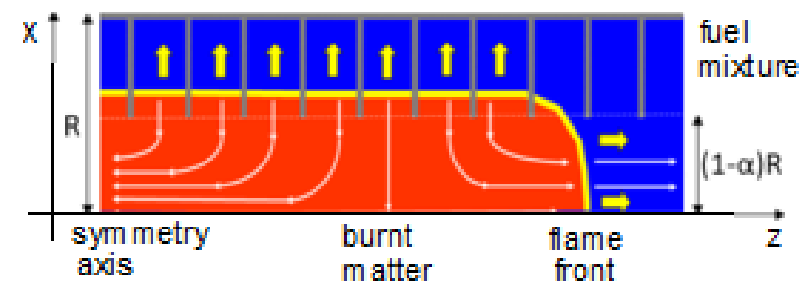

Figure 1.8: A schematic of an obstructed channel with both ends open.

Will the difference between the open and semi-open obstructed channels be as paramount as that for unobstructed ones? The latter question is addressed as part of this dissertation. Specifically, propagation and morphology of a premixed flame front in a channel equipped with a comb-shaped array of obstacles, of various blockage ratios and channel widths, with both ends open are also explored. Furthermore, since energy systems are expected to be highly efficient, producing nearzero emissions, supercritical oxy-fuel combustion with $\mathrm{CO}_{2}$-dilutions has been identified to achieve this objective [23]. In oxy-fuel combustion, the air is substituted with pure oxygen diluted with recycled flue gas consisting primarily of $\mathrm{CO}_{2}$ and $\mathrm{H}_{2} \mathrm{O}$ to moderate the flame temperature as well as eliminating NOx formation. Beyond a critical thermodynamic state, fluids acquire unique thermo-physical properties and, as a result, gases at supercritical state have liquid-like behavior such as relatively high density while liquid would have gas-like properties such as low viscosity. Figure 1.9 shows the pressure-temperature phase diagram for $\mathrm{CO}_{2}$. This property could potentially enhance the combustion efficiencies and inhibit emission formation.

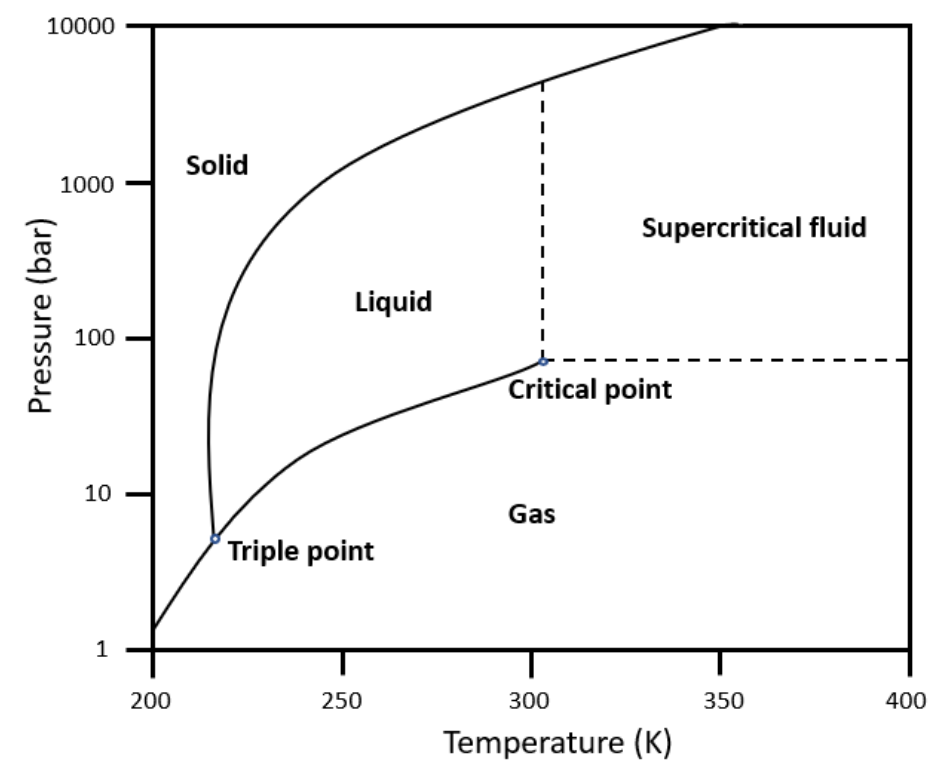

Figure 1.9: A pressure-temperature phase diagram of $\mathrm{CO}_{2}$. 
However, a major drawback of supercritical oxy-combustion is an elevated flame temperature that may degrade reactor materials and cause hydrocarbon coking. To prevent these, the exhaust gases, mainly, supercritical carbon dioxide $\left(\mathrm{sCO}_{2}\right)$ must be recirculated, and it is important to note that supercritical $\mathrm{CO}_{2}$ are chemically stable. The design and development of the future $\mathrm{sCO}_{2}$ combustors for direct-fired cycles require a deeper understanding of the combustion processes under supercritical, highly $\mathrm{CO}_{2}$-diluted conditions. Such combination of these conditions is unique but poses considerably huge challenges to researchers as it remains largely unexplored. As a result, this dissertation explores oxy-methane flame with $\mathrm{CO}_{2}$-dilutions at supercritical conditions of 300 bar and $800 \mathrm{~K}$. The goal here is to provide preliminary insights into the nature of such flames and their propagation rate. Finally, the analysis of supercritical combustion is extended to the Bychkov mechanism. The latter is important because as soon as the novel propulsion systems start taking advantage of thermophysical properties of supercritical fluids, the propagation trends and the morphological nature of the flames in the Bychkov configuration need to be fully understood.

\subsection{Organization of the Dissertation}

This dissertation analyses a collection of computational simulations of premixed flames in obstructed channels (with cylindrical and rectangular cross sections) at atmospheric (ideal gas) and supercritical (real gas) conditions. Chapter 2 provides a review of the recent works on premixed flame propagation in channels. Chapter 3 describes the numerical solution methodology adopted which includes the governing conservation equations, thermodynamic and transport model, a brief description of the meshing scheme and validation of the computational platform as well as description of the parametric studies. Chapter 4 presents the results obtained after scrutinizing the effects of wall shear stress on premixed flame propagation in semi-open obstructed channels. In Chapter 5, the effects of non-equidiffusivity on premixed flame propagation in semi-open obstructed channels are discussed. Chapter 6 explores the oscillation trends in channels with closely-packed obstacles with both ends open, while Chapter 7 characterizes the acceleration regimes observed in such a configuration. Chapters 8 and 9 present the results obtained from simulating supercritical flames in smooth and obstructed channels, respectively. Finally, the conclusions and future works are presented in Chapter 10. 


\section{Literature Review}

\subsection{Premixed Flames in Semi-Open Channels}

The pioneering study in this field, by Chapman \& Wheeler [24], considered an array of obstacles (orifice plates) with one diameter spacing along the walls of a tube. The maximum flame velocity observed in such configuration was $400 \mathrm{~m} / \mathrm{s}$ as compared to $10 \mathrm{~m} / \mathrm{s}$ in the tube without obstacles.

Ciccarelli et al. [25] investigated the impact of obstacles on the early stage of propane-air burning in a $3.05 \mathrm{~m}$ long channel and width of $14 \mathrm{~cm}$. The results obtained shows that the run-up distance (distance to attain sound speed in the unburned fuel mixture) decreases with an increase in the blockage ratio and mixture reactivity. For high blockage ratios, the flame acceleration rate is highest for obstacle spacing equal to one channel width. For a lower blockage ratio, however, acceleration is unaffected when obstacle spacing equal to $0.5,1$ and 1.5 channel width. Moreover, a maximum acceleration rate was observed when the obstacle spacing to height ratio is approximately 5 , and this spacing corresponds to the length of the recirculation zone found ahead of each obstacle in the fuel mixtures.

Johansen \& Ciccarelli [20] visualized the unburned gas flow in a long channel with a square cross-section, filled with obstacles separated by one channel width, to study the influence of the blockage ratio on the initial period of FA for stoichiometric methane-air burning. It was reported that larger blockage ratios favor FA at its early stage, such that higher blockage ratios cause higher acceleration. The visualization shows turbulent flow development in the unburned gases. A vortex develops ahead of each obstacle, then grows until touching the walls of the channel, spanning the spaces between the adjacent obstacles. Thereafter, a shear layer forms separating the core flow from the recirculation zone. This turbulence is responsible for FA, and at higher blockage ratios, turbulence production is enhanced such that the burning rate increases leading to the flame surface area growth and an increase in the flame velocity. It was also observed that the flame tip velocity shows significant oscillations caused by the accelerating and decelerating flows in the unburned gases as it breaks through each obstacle.

Following this experiment, the same authors conducted the large eddy simulations (LES) of methane/air burning [26] to study the flow field in the unburned gas in a square cross-section obstructed channel. The results from this simulation show an initial two-dimensional (2D) laminar 
vortex formed in the unburned gas, which transforms to three-dimension (3D) as the recirculation zone covers the entire spacing between adjacent obstacles. The formation of the recirculation zone causes the regions near the obstacle in the unburned gas to expand and contract thus influencing the flow velocity at the center of the channel. It was also reported that increasing the blockage ratio results in enhancing the rate of turbulence production.

Wang et al. [27] studied methane-air flame propagation in a tube of length $40 \mathrm{~m}$ and diameter $350 \mathrm{~mm}$, obstructed with orifice plates, considering the influence of the obstacle number, spacing and blockage ratio. It was observed for the blockage ratios of 0.3 and 0.6 , with a spacing of 350 $\mathrm{mm}$, that the obstacle number promotes flame acceleration. For the blockage ratio of 0.3 , only the case of 12 obstacles shows sustained acceleration that led to detonation, while in the events of 3 , 6 and 9 obstacles, the flame decelerates after short acceleration. The blockage ratio of 0.6 shows a similar trend to that of 0.3 , however, the flame velocities are higher in this case, with sustained acceleration occurring in the events of both 9 and 12 obstacles. Also, increasing obstacle spacing enhances the flame acceleration rates. Specifically, for the blockage ratio of 0.3 and the obstacle number of 6 , the flame accelerates only when the spacing is increased to $700 \mathrm{~mm}$. In smaller spacing, the flame undergoes deceleration. But with a larger blockage ratio of 0.6, acceleration to detonation occurs for both $700 \mathrm{~mm}$ and $525 \mathrm{~mm}$ spacing. It was concluded that, when obstacles are closely packed, heat losses to obstacles cause a slowdown of the flame.

Dong \& Zhou [28] conducted experiments of methane-air mixtures in a long obstructed tube to understand the effects of obstacles on the overpressure history during the flame acceleration process. It was observed that for a single obstacle placed in a tube, the overpressure time increases as the obstacle is moved away from the point of ignition while the peak pressure is unaffected. However, with multiple obstacles, the observed peak pressure increases as the number of obstacles increases.

Porowski \& Teodorczyk [29], conducted the experiments of hydrogen-air burning in an obstructed tube of $6 \mathrm{~m}$ long, $140 \mathrm{~mm}$ diameter, with methane additions. The results show that for the largest blockage ratio considered, 0.7, the flame accelerates, and DDT occurs only for the hydrogen/air mixtures. With methane addition to the hydrogen-oxygen mixtures, for a tube with a blockage ratio of 0.7 , the flame accelerated, but this did not result in a detonation. However, FA and DDT for methane-hydrogen/air mixture occur for the lower blockage ratios of 0.4 and 0.6. 
The transition to detonation occurs with blockage ratio of 0.4 , in the case of methane addition to the hydrogen/air flame up to 50\%. Moreover, the transition to detonation is favored by increasing the number of obstacles until the spacing between the obstacles equals to one diameter.

A 2D numerical simulation of stoichiometric hydrogen/air flame with one-step Arrhenius chemical kinetics in an obstructed channel was conducted by Gamezo et al. [10], to scrutinize the influence of obstacle spacing on FA and DDT. The results show that increasing the number of obstacles per unit length increases the flame acceleration rate. However, the detonation happens faster at larger obstacle spacing, when the spacing is large enough for the Mach stem formation. It was understood that the Mach stem formation happens at wider obstacle spacing, when diffracting shocks from the walls collide, with obstacles producing hot spots in the unburned fuel mixtures that trigger detonation.

Boeck et al. [30] utilized OH-PLIF imaging techniques and 2D simulation with detailed chemistry to study acceleration of hydrogen/oxygen mixtures in a channel with a square crosssection and a single obstacle. It was reported that the flame accelerates after passing through the obstacle and attains a maximum velocity of $590 \mathrm{~m} / \mathrm{s}$, before decelerating far away from the obstacle. Also, strong flame-vortex interaction in the recirculating region ahead of the obstacle wrinkles the flame significantly. While the results of the numerical simulation predict the flame tip velocity accurately, the flame surface wrinkling was over-predicted.

Kessler et al. [31] carried out the simulation of methane-air flame acceleration and transition to detonation in an obstructed channel. It was observed that flame acceleration occurs in three phases: starting with an elongation of the flame front, it gets wrinkled due to turbulence and flow instabilities and then the flame surface gets recreated due to shock interference. It was also observed that geometry does not affect the observed saturated velocity otherwise referred to as choked velocity, but the fuel mixture properties influence this saturated velocity.

Boeck et al. [32] carried out a detailed characterization of FA in an obstructed channel with a square cross-section, utilizing both the OH-PLIF and Schlieren techniques for hydrogen/oxygen mixtures considering various obstacle numbers. In the case of one obstacle, at $25 \mathrm{kPa}$, the flame tip accelerates towards the obstacles opening, as the flow contracts through the opening. Acceleration continues downstream of the opening until the flame tip velocity reached $690-720$ $\mathrm{m} / \mathrm{s}$ and decelerates shortly to $340-515 \mathrm{~m} / \mathrm{s}$, after which it accelerates again due to the turbulent 
burning in the recirculation zone. In the presence of three obstacles, the flame accelerates to a maximum velocity of $1000-1030 \mathrm{~m} / \mathrm{s}$ and undergoes a series of acceleration and deceleration stages. The formation of shock wave causes small scale wrinkling only in the skirts of the flame. As the obstacle number increases to 11 , the flame reaches a peak velocity of $1150 \mathrm{~m} / \mathrm{s}$ and attains quasi-steady propagation further downstream with apparent flame shock interaction. Flame acceleration in obstructed channels was summarized to comprise of three stages. First, the initial period of slow flame acceleration controlled by thermal expansion, flame area growth, and turbulence interaction. Next, a shock wave develops causing flame velocity oscillation, and smallscale corrugation. The later stage consists of high-speed, near-steady propagation, where shock wave interaction leads to flame deformation and significant small-scale wrinkling.

Teodorczyk et al. [33] conducted the experiments on hydrogen/air burning in a channel with a square cross-section, with obstacles placed only at the bottom wall. It was observed in this configuration that increasing blockage ratio creates a momentum loss. Also, a larger blockage ratio increases the obstacle spacing distance required to maximize the acceleration rates. It was concluded that the obstacles density is less significant in accelerating lean hydrogen mixtures.

Ciccarelli et al. [14] investigated the later stage of FA for methane/air burning in a channel with a square cross-section. It was reported that the presence of the shear layer in the spacing between the obstacles prevents instantaneous burning of the gases and thereby causing the flame skirts to extend further upstream towards the point of ignition, and the elongated flame surface area supports FA. In contrast, the later region of acceleration is dictated by the compression waves and flame-shock interaction. Moreover, such a flame-shock interaction is unique for each blockage ratio. As a shock wave contacts the walls and obstacles, its reflection coalesces with the flame, thereby, influencing it significantly. Initially, when the leading shock wave lies further ahead of the flame, the reflected shock retards the flame and causes an initial recession of flame velocity. However, as the flame moves closer to the leading shock, the reflected shock is unable to slow down the flame tip velocity. But, the interaction of the flame with the shock reflection produces an oscillation in the flame tip velocities, after which the flame attains a near-steady propagation velocity also called the choked velocity. This saturated speed depends on the blockage ratio and it increases as the blockage ratio decreases such that at the blockage ratio of 0.33 the saturated speed approaches the speed of sound in the burned gases. 
After the detailed computational simulations and the analytical studies of FA by Bychkov et al. [15], it was discovered that a conceptually distinctive acceleration mechanism occurs in obstructed channels having a closely packed (say, a tooth-brush-like) array of thin obstacles, such that the obstacle spacing is much less than the channel half-width. As the flame front propagates due to thermal expansion, it leaves behind unburned gas in the pockets formed by the adjacent obstacles. Eventually, burning in these pockets occurs, producing a jet flow in the core of the channel that drifts the flame front further ahead. The main driving force of this acceleration is the jet-flow, with turbulence playing only a supplementary role. This acceleration mechanism is Reynolds-independent (scale-invariant). The theoretical formulation predicting the flame tip propagation was developed for a flame in a 2D rectangular domain at the early stage of burning. The simplifying assumption made are: (i) incompressibility, (ii) negligible obstacle thickness, (iii) an infinitely thin flame, (iv) slip walls and obstacles such that the flame propagation in the pockets is not corrugated but planar, (v) $L e=1$. The formulation is described as

$$
\begin{gathered}
\frac{Z_{f}}{(1-\alpha) R}=\frac{\Theta}{\Theta-1}\left[\exp \left(\sigma S_{L} t / R\right)-1\right], \\
\sigma=\frac{\Theta-1}{1-\alpha},
\end{gathered}
$$

where $\sigma$ represents the scaled acceleration rate depending on the thermal expansion ratio $\Theta$ and the blockage ratio $\alpha$. As expressed in the analytical formulation and validated by the computational simulations, FA is exponential in time.

Valiev et al. [16] explored the Bychkov mechanism further, studying FA and DDT in tubes with closely packed obstacles. In particular, the theory of this mechanism was extended to the axisymmetric cylindrical configuration. The exponential acceleration rate in this geometry is twice the rate for the 2D case. It was also reported that FA weakens as the initial Mach number increases. At the later stage of acceleration, the flame velocity saturates to a quasi-steady value, lower than the Chapman-Jouguet deflagration speed. It was also observed that FA in this mechanism can trigger detonation downstream of the flame.

The early stage of flame propagation according to the Bychkov acceleration mechanism was validated experimentally [19] for methane/air burning in a channel of $2 \mathrm{~m}$ long, with a square cross-section and closely packed obstacles. The study employed the channels with the obstacle spacing of $1 / 16$ of channel width and blockage ratio of $2 / 3$. The results of the flame tip 
propagation with time matches the analytical formulation of Bychkov quite well, however, the flame tip velocity starts deviating from the theory after a flame has traveled about four channel widths. The assumption of a planar flame in the pockets was, however, invalidated as vortices are formed at the entrance of the pockets.

Bychkov et al. [17] extended the original incompressible formulation to the later stage of burning, where compressibility effects become significant. It was reported that gas compressibility moderates flame acceleration. This moderation is a combination of linear and non-linear effects. The linear effect reduces the exponential acceleration rates at the beginning, while the nonlinear effect becomes significant away from the closed end of the channel. An analytical formulation for the flame tip propagation, developed considering finite gas compression, reads

$$
\begin{gathered}
Z_{f}=\frac{2 \Theta_{1} S_{L}\left[\exp \left(\sigma_{1} t\right)-1\right]}{\left(\sigma_{2}-\sigma_{1}\right) \exp \left(\sigma_{2} t\right)+\left(\sigma_{2}+\sigma_{1}\right)}, \\
\sigma_{2}=\sqrt{\sigma_{1}^{2}+4 M a \chi \Theta_{1} \sigma_{0}^{2}}, \\
\sigma_{1}=\sigma_{0}\left[1-M a\left(\frac{\Theta}{1-\alpha}+2(\gamma-1)(\Theta-1)\right)\right],
\end{gathered}
$$

where $M a=S_{L} / c_{0}$ is the initial flame Mach number, associated with flame propagation, and $\gamma=$ $c_{P} / c_{v}$ is the specific heat ratio. The cylindrical-axisymmetric counterpart of this formulation, developed by Akkerman \& Valiev [21], reads

$$
\begin{gathered}
Z_{f}=\frac{2 \psi S_{L}\left[\exp \left(\sigma_{1} t\right)-1\right]}{\left(\sigma_{2}-\sigma_{1}\right) \exp \left(\sigma_{2} t\right)+\left(\sigma_{2}+\sigma_{1}\right)}, \\
\psi=1-M a(\gamma-1)(\Theta-1)^{2}, \\
\sigma_{2}=\sqrt{\sigma_{1}^{2}+4 M a x \psi \Theta_{1} \sigma_{0}^{2}} .
\end{gathered}
$$

The influence of gas compressibility is much stronger in a cylindrical channel as compared to a rectangular channel. Extensive parametric study of the Bychkov mechanism was conducted by Ugarte et al.[18], considering the influence of the blockage ratio, obstacle spacing, channel width, ignition type and the thermal boundary condition. The result of this simulation shows that the blockage ratio is the most significant and influential parameter driving flame acceleration in this domain. As the blockage ratio increases, the flame tip velocity grows accordingly. Moreover, for the blockage ratio $\alpha=1 / 40 \sim 1 / 3$, the corresponding limit of exponential acceleration was estimated analytically and numerically to be $\alpha=1 / 8$ and $1 / 9$ respectively. The difference in the 
acceleration trend for lower blockage ratios, $\alpha=1 / 5 \sim 1 / 20$, is minor. Variations of the channel width did not show any effect on acceleration as such the assumption of Reynolds-independence was validated. However, increasing obstacle spacing reduces the acceleration rate. It was observed that as the obstacle spacing increases, vortices emerge in the pockets, which invalidates the concept of the Bychkov mechanism. However, the role of obstacle spacing is minor at low blockage ratios. The effect of both isothermal and adiabatic boundary conditions was also studied considering both cold and preheated isothermal walls. In all thermal boundary conditions tested, the flame geometry and propagation rates were not affected significantly. Finally, the ignition type was also studied considering both point and planar ignition, and both gives similar results.

\subsection{Premixed Flames in Channels with Both Ends Open}

Yanez et al. [34], studied the nature of hydrogen/air flame propagation in vented obstructed channels. It was observed that the flame initially undergoes near-laminar propagation, with a propagation velocity being close to the laminar flame speed (i.e. no acceleration). However, after some interval of time, the flame undergoes fast, rapid acceleration. This sudden acceleration was reported to happen without volumetric expansion, shock or shock-wave interaction processes. It was concluded that rapid acceleration is caused by hydraulic resistance imposed when the burnt gas exits the channel, and this resistance increases with channel lengths.

Middha \& Hansen [35], observed similar sudden acceleration in the computational fluid dynamics (CFD) simulation of a hydrogen explosion in a channel with both ends open. However, the details of the physics behind the phenomenon were not discussed.

Bychkov et al. [36], developed an analytical formulation for flame propagated in obstructed channels with both ends opened. The theorical formulation for inviscid flows is presented below. Namely, the flame tip position $Z_{f}$ and the scaled acceleration rate $\sigma_{0}$ for inviscid flow are

$$
\begin{gathered}
Z_{f}=\frac{\Theta R}{\sigma_{0}}\left\{\exp \left(\sigma_{0} S_{L} t / R\right)-1\right\}, \\
\sigma_{0}=\frac{\Theta-1}{\sqrt{(\Theta+1)}(1-\alpha)} .
\end{gathered}
$$

When viscous effects are considered, the flame tip position is obtained from the equations [37]

$$
U_{1} \frac{\cosh \mu_{1}-\mu_{1}^{-1} \sinh \mu_{1}}{\cosh \mu_{1}-1}+U_{2} \frac{\cosh \mu_{2}-\mu_{2}^{-1} \sinh \mu_{2}}{\cosh \mu_{2}-1},
$$




$$
\begin{gathered}
\frac{U_{1}^{2}}{\Theta S_{L}^{2}}+\frac{\mu_{1} \sinh \mu_{1}}{\cosh \mu_{1}-1} \frac{U_{1} Z_{f}}{(1-\alpha)^{2} R e S_{L} R}=\frac{U_{2}^{2}}{\Theta S_{L}^{2}}+\Theta-1+\frac{\mu_{2} \sinh \mu_{2}}{\cosh \mu_{2}-1} \frac{U_{2}\left(L-Z_{f}\right)}{(1-\alpha)^{2} R e S_{L} R}, \\
\frac{d Z_{f}}{d t} \approx \frac{\sigma S_{L}}{R}\left[Z_{f}+\frac{T}{\sigma}\right] \approx U_{2}\left(Z_{f}, R e\right)+S_{L} .
\end{gathered}
$$

It was reported that, the hydraulic resistance is not needed to accelerate the flame because it only influences the region of quasi-steady propagation.

\subsection{Premixed Flames with $\mathrm{CO}_{2}$-dilutions}

The literatures on the impacts of the supercritical $\mathrm{CO}_{2}$-dilutions on premixed flames are presently scarce. However, the topic has been extensively studied at the atmospheric conditions (or at the typical gas turbine pressure levels) experimentally/numerically, for various fuel mixtures based on $\mathrm{CH}_{4}$ or $\mathrm{H}_{2} / \mathrm{CO}$ (syngas). In $\mathrm{CH}_{4} / \mathrm{O}_{2}$ mixtures with $\mathrm{CO}_{2}$, Sun et. al. [37] demonstrated that while $\mathrm{CO}_{2}$ may not affect the major reaction pathways, it does have some minor effects on the reactivity. Yang et al. [38] reported that $\mathrm{H}_{2} \mathrm{O}$ diluents produced the highest $\mathrm{OH}$ number density, followed by $\mathrm{N}_{2}$, and with $\mathrm{CO}_{2}$ producing the lowest $\mathrm{OH}$ number density. It was also reported that the flame temperature decreases more with $\mathrm{CO}_{2}$ as compared to $\mathrm{H}_{2} \mathrm{O}$ and $\mathrm{N}_{2}$ dilutions. For $\mathrm{CH}_{4} / \mathrm{CO}_{2} / \mathrm{O}_{2}$ at the atmospheric pressure and the room temperature, major reaction pathways were unaffected by the $\mathrm{CO}_{2}$-dilution, however, the secondary reaction pathways and the rates of intermediate species formation from elementary species were altered. Moreover, $\mathrm{CO}_{2}$-dilution was observed to decrease some important intermediate reactants and product concentration. $\mathrm{CO}_{2}$-dilution of syngas flames causes the dissociation reaction which leads to the consumption of the H-radicals, and their extinction limits and temperature increases with reaction pressure [39].

In syngas flames, $\mathrm{CO}_{2}$ generally has a stronger thermal and chemical influence on lowering reaction temperature and concentration of radicals [40]. According to the study of methane/air combustion with $\mathrm{CO}_{2}$, increasing $\mathrm{CO}_{2}$ concentration causes reduction of the temperature of the reactants, the net rate of reaction and the speed of the flame [41]. Chan et al. [42] also reported that for laminar methane/air flames, increasing the $\mathrm{CO}_{2}$-dilution concentration decreases the flame speed accordingly, and $\mathrm{CO}_{2}$ absorbs heat from reactants species, reducing a tendency to attain the activation temperature. According to the work of Yang et al. [43], $\mathrm{CO}_{2}$ has a stronger impact on the laminar flame speed and extinction limits than $\mathrm{N}_{2}$ diluted syngas flame and the thermal effects created by $\mathrm{CO}_{2}$-diluents dominate the decrease in the flame speed, while the chemical effects 
marginally cause a stronger influence than the thermal effect on the reduction of the flame extinction limits.

In methane/air premixed flames, $\mathrm{CO}_{2}$ has a greater effect on the flame thermal properties than $\mathrm{N}_{2}$, and it also has significant effects on the inhibition of NO formation [44]. Khan et al. [45] studied the effects of $\mathrm{N}_{2} / \mathrm{CO}_{2}$ on the laminar burning velocity for freely-expanding, oxy-methane flames. With $50 \% \mathrm{CO}_{2}$ diluents, the burning velocity decreases by $88 \%$ as compared to the $\mathrm{CH}_{4} / \mathrm{O}_{2}$ case, and it is 3.6 times less than that for the flame having $50 \% \mathrm{~N}_{2}$. For $\mathrm{CH}_{4} /$ air mixture at $1.0 \mathrm{MPa}$ and $573 \mathrm{~K}$, the exhaust gas recirculation consisting mainly of $\mathrm{CO}_{2}$ was identified to have a significant impact on the nature of turbulent premixed flames according to Kobayashi et al. [2]. It was reported that the turbulent burning velocity and fuel consumption rates decrease with $\mathrm{CO}_{2}$ dilution rates while the average volume of the flame region increases with dilution. Moreover, the addition of $\mathrm{CO}_{2}$ moderates flame instability by decreasing the smallest scale of flame wrinkles. In a similar study, Kobayashi et al. [46] conducted comparative study of the $\mathrm{CO}_{2}$-diluted syngas and methane gas combustion at 1.0 MPa. It was reported that the flame surface density of the $\mathrm{CO}_{2}$ diluted syngas $\left(\mathrm{CO} / \mathrm{H}_{2}\right) / \mathrm{O}_{2}$ mixture exceeds that of methane/air and syngas-air flames. However, a $\left(\mathrm{CO} / \mathrm{H}_{2} / \mathrm{CO}_{2}\right) / \mathrm{O}_{2}$ flame has the turbulent burning velocity and large-scale wrinkles superimposed on the small-scales that are much smaller than methane/air flames. It was also observed that the total radiation intensity of diluted $\left(\mathrm{CO} / \mathrm{H}_{2} / \mathrm{CO}_{2}\right) / \mathrm{O}_{2}$ flames are about 1.6 of that of methane/air. 


\section{Research Methodology}

The research methodology employed in this dissertation is based on analyzing reacting flows in channels by means of computational simulation of various governing equations which are sets of partial differential equations modeling mass, momentum, energy and species transport. The dimension of the problems is reduced to $2 \mathrm{D}$ for rectangular channels and axisymmetric $2 \mathrm{D}$ for cylindrical channels with the length scales limited to micro-scales.

\subsection{Governing Equations}

The conservation equation of mass, momentum, energy and species read

$$
\begin{gathered}
\frac{\partial \rho}{\partial t}+\frac{1}{r^{\omega}} \frac{\partial}{\partial r}\left(r^{\omega} \rho u_{r}\right)+\frac{\partial}{\partial z}\left(\rho u_{z}\right)=0 \\
\frac{\partial}{\partial t}\left(\rho u_{r}\right)+\frac{1}{r^{\omega}} \frac{\partial}{\partial r}\left[r^{\omega}\left(\rho u_{r}^{2}-\zeta_{r, r}\right)\right]+\frac{\partial}{\partial z}\left(\rho u_{\mathrm{r}} \mathrm{u}_{\mathrm{z}}-\zeta_{r, z}\right)+\frac{\partial P}{\partial r}+\Psi_{\omega}=0 \\
\frac{\partial}{\partial t}\left(\rho u_{z}\right)+\frac{1}{r^{\omega}} \frac{\partial}{\partial r}\left[r^{\omega}\left(\rho \mathrm{u}_{\mathrm{r}} \mathrm{u}_{\mathrm{z}}-\zeta_{r, z}\right)\right]+\frac{\partial}{\partial z}\left(\rho u_{z}^{2}-\zeta_{z, z}\right)+\frac{\partial P}{\partial z}=0 \\
\frac{\partial \varepsilon}{\partial t}+\frac{1}{r^{\omega}} \frac{\partial}{\partial r}\left[r^{\omega}\left((\varepsilon+P) u_{r}-\zeta_{r, r} u_{r}-\zeta_{r, z} u_{z}+q_{r}\right)\right]= \\
-\frac{\partial}{\partial z}\left[(\varepsilon+P) u_{z}-\zeta_{z, z} u_{z}-\zeta_{r, z} u_{r}+q_{z}\right] \\
\frac{\partial}{\partial t}(\rho Y)+\frac{1}{r^{\omega}} \frac{\partial}{\partial r}\left[r^{\gamma}\left(\rho u_{i} Y-\frac{\eta}{\mathrm{Sc}} \frac{\partial Y}{\partial r}\right)\right]+\frac{\partial}{\partial z}\left(\rho u_{z} Y-\frac{\eta}{\mathrm{Sc}} \frac{\partial Y}{\partial z}\right)=-\frac{\rho Y}{\tau_{R}} \exp \left(-E_{a} / R_{u} T\right),
\end{gathered}
$$

where $\omega=0$ and $\omega=1$ for $2 D$ and axisymmetric geometries respectively, and the primary variables are the density $\rho$, velocity $u$, pressure $P$, and total energy per unit volume $\varepsilon$,

$$
\varepsilon=\rho\left(Q Y+C_{V} T\right)+\frac{\rho}{2}\left(u_{z}^{2}+u_{r}^{2}\right)
$$

where $Y$ is the mass fraction of the fuel mixture, $Q$ is the energy release in the reaction, and $C_{V}$ is the specific heat capacity at constant volume. The Arrhenius reaction is the $1^{\text {st }}$-order, where the activation energy and the characteristic time constant corresponding to the inverse of the preexponential factors are $E_{a}$ and $\tau_{P}$, respectively. The energy diffusion vector $q_{i}$ is given by

$$
q_{r}=-\eta\left(\frac{C_{P}}{P r} \frac{\partial T}{\partial r}+\frac{Q}{S c} \frac{\partial Y}{\partial r}\right), \quad q_{z}=-\eta\left(\frac{C_{P}}{P r} \frac{\partial T}{\partial \mathrm{z}}+\frac{Q}{S c} \frac{\partial Y}{\partial \mathrm{z}}\right),
$$

were $\eta$ is the dynamic viscosity, and $\operatorname{Pr}$ and Sc the Prandtl and Schmidt numbers, respectively, The stress tensor $\zeta_{i, j}$ takes the form 


$$
\zeta_{i, j}=\eta\left(\frac{\partial u_{i}}{\partial x_{j}}+\frac{\partial u_{j}}{\partial x_{i}}-\frac{2}{3} \frac{\partial u_{k}}{\partial x_{k}} \delta_{i, j}\right)
$$

in the $2 \mathrm{D}$ configuration $(\omega=0)$, while in the axisymmetric geometry $(\omega=1)$ it reads

$$
\zeta_{r, r}=\frac{2 \eta}{3}\left(2 \frac{\partial u_{r}}{\partial r}-\frac{\partial u_{z}}{\partial z}-\frac{u_{r}}{r}\right), \zeta_{z, z}=\frac{2 \eta}{3}\left(2 \frac{\partial u_{z}}{\partial z}-\frac{\partial u_{r}}{\partial r}-\frac{u_{r}}{r}\right), \zeta_{r, z}=\eta\left(\frac{\partial u_{r}}{\partial z}+\frac{\partial u_{z}}{\partial r}\right) .
$$

Finally, the last term in Eq. (3.2) takes the form

$$
\Psi_{\omega}=\frac{2 \eta}{3}\left(2 \frac{u_{r}}{r}-\frac{\partial u_{r}}{\partial r}-\frac{\partial u_{z}}{\partial z}\right) \text { if } \omega=1 \text {, and } \psi_{\omega}=0 \text { if } \omega=0
$$

\subsection{The Solution Scheme}

The sets of governing equations are solved using an in-house solver based on the finite-volume discretization scheme. After discretizing the equations, the resulting ordinary differential equations are solved explicitly with the $4^{\text {th }}$-order Runge-Kutta method [47] implemented in Fortran and C programming languages. The original versions of the code are based on the ideal gas equation of state (IG EoS), with the Sunderland formulation used to calculate the viscosity and considered several simplifications. To study combustion at supercritical conditions, the code was modified extensively to incorporate effect of temperature and pressure on the thermodynamic and transport properties. Both the original implementation and the modified version developed for this work are presented next.

\subsubsection{Ideal Gas Model - Original Solver}

In this model, the fuel mixtures and the burned product are assumed to be ideal gases of the same molar weight being $M=0.029 \mathrm{~kg} / \mathrm{mol}$, and the specific heat at constant pressure and temperature are calculated from the gas kinetic theory as $C_{V}=5 R_{u} / 2 M$ and $C_{P}=7 R_{u} / 2 M$. From the IG EoS, the pressure $P=\rho R_{u} T / M$, where $R_{u}$ is the universal gas constant. The energy release in the chemical reaction is given by $Q=(\Theta-1) C_{P} T_{f}$, where $T_{f}$ is the cold fuel mixture temperature. However, the effect of radiative heat transfer is not modeled into the solver.

\subsubsection{Real Gas Model - Modified Solver}

Both the thermodynamic and transport properties are modified to account for the real gas effect at supercritical conditions. The thermodynamic properties are modeled based on the Peng-Robinson cubic equation of state (PR EoS) [48] 


$$
P=\frac{R_{u} T}{v-b}-\frac{a}{v(v+b)+b(v-b)},
$$

where $v$ is the molar volume, $R_{u}$ is the universal gas constant, and the equation parameters $a, b$ are defined based on the quadratic and linear mixing rules [48]

$$
a=\sum_{i=1}^{N} \sum_{j=1}^{N} X_{i} X_{j} \sqrt{a_{i} a_{j}\left(1-k_{i, j}\right)}, \quad b=\sum_{i=1}^{N} X_{i} b_{i},
$$

$N$ is the number of species in the mixture, $X_{i}$ the mole fraction of species $i$, and $k_{i j}$ the binary interaction parameters, namely, the empirical quantities describing the interactions between the binary species such that $k_{i, j}=0$ if $i=j$, while for all $i \neq j$, these values are $k_{\mathrm{CH} 4-\mathrm{CO} 2}=0.0919$, $k_{\mathrm{CH} 4-\mathrm{O} 2}=0.03, k_{\mathrm{CH} 4-\mathrm{H} 2 \mathrm{O}}=0.485, k_{\mathrm{CO} 2-\mathrm{O} 2}=0.0015, k_{\mathrm{H} 2 \mathrm{O}-\mathrm{O} 2}=0.49, k_{\mathrm{CO} 2-\mathrm{H} 2 \mathrm{O}}=0.1896$ [49-50]. The quantities $a_{i}, b_{i}$ for each individual species are given by

$$
\begin{array}{r}
a_{i}=0.45724 \frac{R_{u}^{2} T_{c, i}^{2}}{P_{c, i}}\left[1+\left(0.37464+1.5422 s_{i}-0.26992 s_{i}^{2}\right)\left(1-T_{r, i}^{1 / 2}\right)\right]^{2}, \\
b_{i}=0.0778 \frac{R_{u} T_{c, i}}{P_{c, i}},
\end{array}
$$

where $T_{c, i}, P_{c, i}$ are the critical temperature and pressure for each species, $T_{r, i}=T_{c, i} / T$ the reduced temperature and $s_{i}$ the Pitzer's acentric factor. The specific heats functions are expressed as

$$
C_{v}=C_{v \mid \text { ideal }}+C_{v \mid \text { residual },} \quad C_{p}=C_{p \mid \text { ideal }}+C_{p \mid \text { residual }},
$$

The IG components are computed from the NASA polynomial approximation [51] while residual components are given by [52]

$$
C_{v, \text { residual }}=\int_{\infty}^{v} T\left(\frac{\partial^{2} p}{\partial T^{2}}\right)_{v} d v, \quad C_{p, \text { residual }}=\int_{\infty}^{v} T\left(\frac{\partial^{2} p}{\partial T^{2}}\right)_{v} d v-\frac{T\left(\frac{\partial p}{\partial T}\right)_{v}^{2}}{\left(\frac{\partial v}{\partial T}\right)_{p}}-R,
$$

The energy release in the chemical reaction is

$$
Q=\int_{T_{f}}^{T_{a d}} C_{p}(T) d T .
$$

The effect of supercritical conditions on the transport properties are accounted for using the Lucas viscosity model for dense gases mixture [53] given by

$$
\eta=\frac{Z_{2} F_{P_{m}}}{\xi} \times 10^{-7}
$$


with the reduced low-pressure inverse viscosity $\xi$,

$$
\xi=0.176\left(\frac{T_{c_{m}}}{T_{c_{m}} M_{m}^{3} P_{c_{m}}^{4}}\right)^{1 / 6} .
$$

A parameter accounting for the pressure effect is

$$
Z_{2}=Z_{1}\left[1+\frac{a P_{r_{m}}^{e}}{b P_{r_{m}}^{f}+\left(1+c P_{r_{m}}^{d}\right)^{-1}}\right],
$$

With a parameter accounting for the temperature effects being

$$
Z_{1}=\left[0.807 T_{r_{m}}^{0.618}-0.357 \exp \left(-0.449 T_{r_{m}}\right)+0.34 \exp \left(-4.058 T_{r_{m}}\right)+0.018\right]
$$

A correction factor $F_{P_{m}}$ in Eq. (3.18) takes the form

$$
F_{P_{m}}=\frac{\left(1.0+\left(F_{p_{0 m}}-1.0\right)\left(Z_{1} / Z_{2}\right)^{3}\right)}{F_{p_{0_{m}}}} .
$$

The mixture properties $T_{c_{m}}, P_{c_{m}}, M_{m}, P r_{m}, T r_{m}, F_{p_{0_{m}}}$ for the temperature, pressure, molecular weight, reduced pressure, reduced temperature, and low pressure polarity are calculated from the sum of the products of the species mole fraction and the properties over all species in the mixture. The values $a, b, c, d, e, f$ are parameters of the Lucas model [53]. The plots of the viscosity calculation from Eq. 3.18 of stochiometric oxy-methane mixture at $T_{f}=800 \mathrm{~K}$, as it depends on pressure is shown in Fig. 3.1a. Fig. 3.1b shows the influence of $\mathrm{CO}_{2}$-dilutions on the viscosity of stochiometric oxy-methane mixture at $\mathrm{T}_{\mathrm{f}}=800 \mathrm{~K}$ and $P_{\mathrm{f}}=300$ bar. The Fortran implementation of the Peng Robinson EoS and the Lucas model can be found in Appendix A and B, while the C codes are in Appendix C and D for the PR EoS and the Lucas models respectively.

(a)

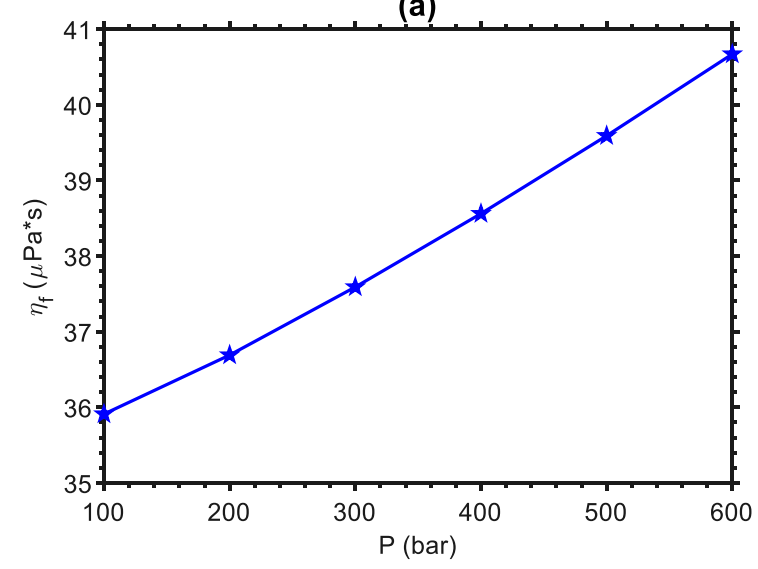

(b)

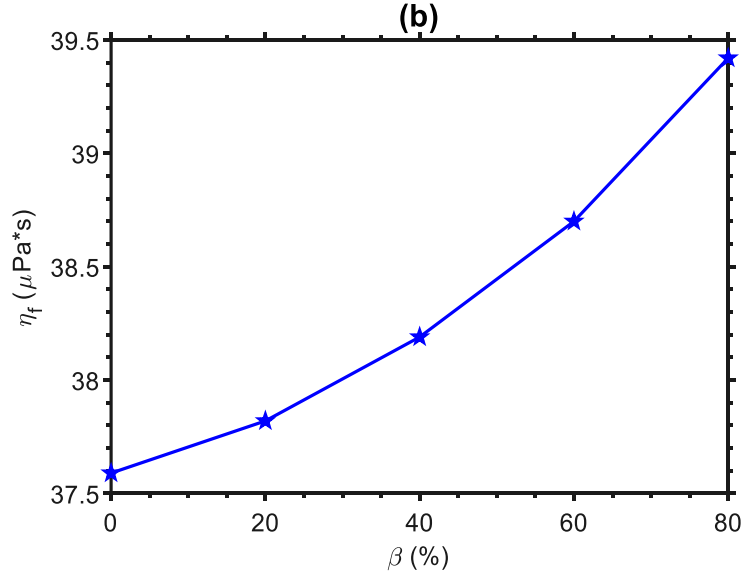

Figure 3.1: Viscosity at $T_{f}=800 \mathrm{~K}$ for a) $\beta=0 \%$ b) $P_{f}=300 \mathrm{bar}$. 


\subsection{Grid/Mesh Generation}

The computational mesh consists of several subdomains depending on the version of the solver. These subdomains include the uniform mesh covering the extent of the flame plus margins before and after the flame, allowing for the movement of the flame. The same is for the leading wave, but the grid size for such a wave is usually larger. The mesh is shown in Fig. 3.2; it is self-adaptive and grows dynamically tracking the flame as well as the leading shock wave. As a result, the mesh is recreated regularly, and the old data set is interpolated onto the new mesh. The mesh generation algorithm and pre-processing unit of the code is implemented in $\mathrm{C}$ programming language.

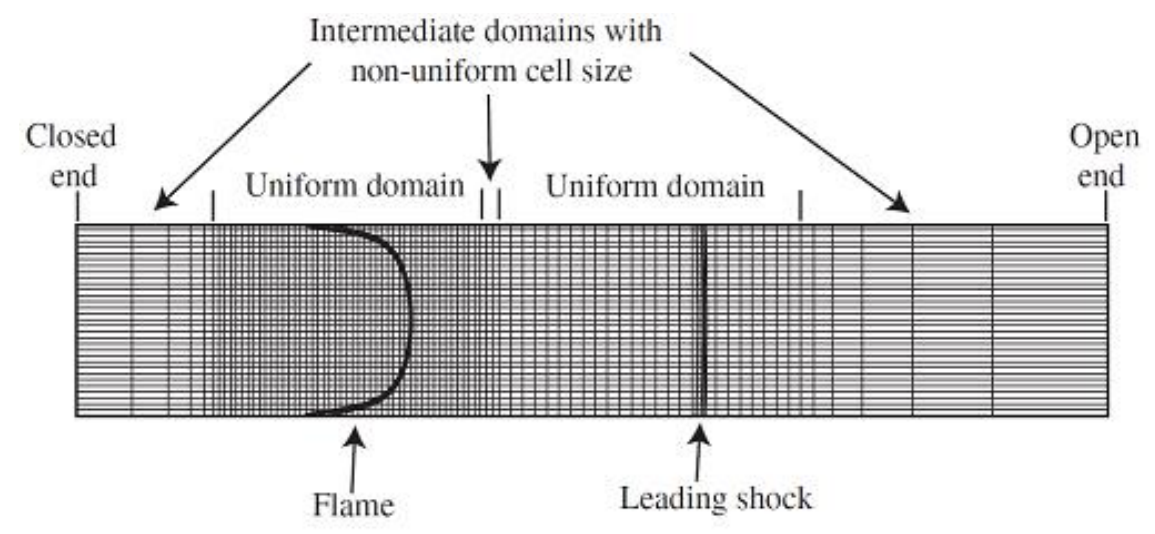

Figure 3.2: Schematic of the grid used in the numerical simulations.

\subsection{The Ignition Model}

The initial flame structure is imitated by the Zeldovich-Frank-Kamenetskii (ZFK) solution $[55,56]:$

$$
Y=\left(\Theta-T / T_{f}\right) /(\Theta-1), \quad P=P_{f}, \quad u_{x}=0, \quad u_{z}=0
$$

such that the fuel mixture mass fraction (or the reaction progress variable) $Y$ is $Y=0$ in the burnt matter and $Y=1$ in the fresh fuel mixture. The pressure $P$ in the channel is equal to the initial fuel mixture $P_{f}$ and the velocity components are set to zero at the beginning. Such a solution is shown for both planar and spherical ignition in Figs. 3.3 and 3.4, respectively. The temperature profiles in the domain before and after a planar flame front are

$$
\begin{aligned}
T=T_{f}+T_{f}(\Theta-1) \exp \left(z / L_{f}\right) & \text { if } \quad z>0 \\
T=\Theta T_{f} & \text { if } \quad z<0
\end{aligned}
$$




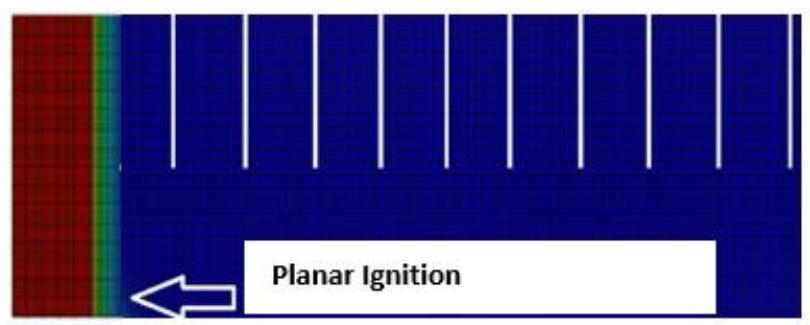

Figure 3.3: Solution initialization based on a planar ignition.

For a hemispherical flame, the temperature profile in the domain created from a point ignition is

$$
\begin{array}{r}
T=T_{f}+\left(T_{b}-T_{f}\right) \exp \left(\left(-\sqrt{\left(x^{2}+z^{2}\right)}+r_{f}\right) / L_{f}\right) \quad \text { if } \quad z^{2}+x^{2} \geq r_{f}^{2} \\
T=\Theta T_{f} \quad \text { if } \quad z^{2}+x^{2}<r_{f}^{2}
\end{array}
$$

where $r_{f}$ is the initial flame radius position and $T_{b}$ is the temperature of the burned matter.

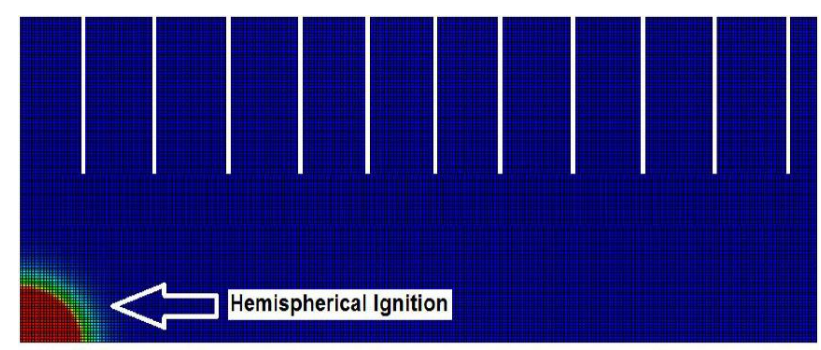

Figure 3.4: Solution initialization based on a hemispherical ignition.

\subsection{Validations}

\subsubsection{Smooth Channels}

The ideal gas version of the code has been previously validated [56] by experiment on ethylene-oxygen burning in a smooth capillary tube of diameter $0.25 \mathrm{~cm}$ and $1.5 \mathrm{~m}$ long. A high voltage electrode was used to ignite the mixture as shown in the experimental set up in Fig. 3.5. The basic parameters used to simulate the fuel are the kinematic viscosity $v=1.72 \times 10^{-5} \mathrm{~m}^{2} / \mathrm{s}$, the planar flame speed $S_{L}=5.1 \mathrm{~m} / \mathrm{s}$, specific heat ratio $\gamma=1.34$. The flame tip velocity is shown in Fig. 3.6. Both the numerical scheme and solver give good prediction of the flame propagation trends. 


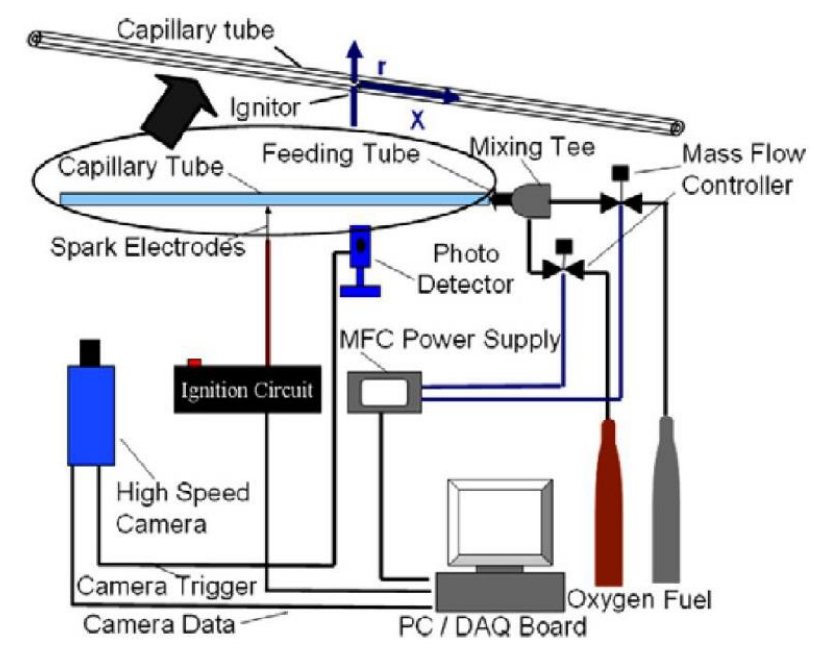

Figure 3.5: Experimental set-up of ethylene-oxygen mixture in a capillary tube [57].

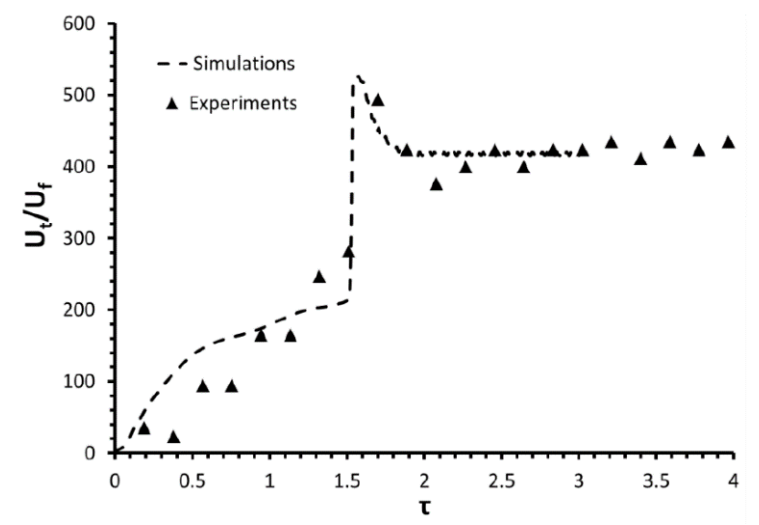

Figure 3.6: The flame tip evolution in a tube of radius $0.25 \mathrm{~mm}$ (experiment versus simulations [57]).

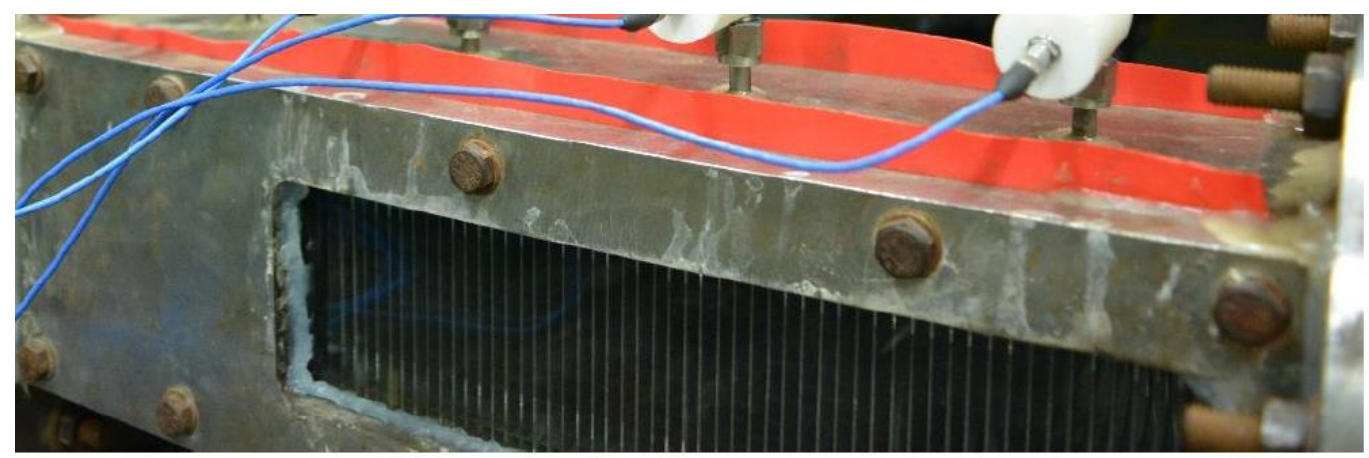

Figure 3.7: Optical access to combustion channel with closely packed obstacles [19]. 


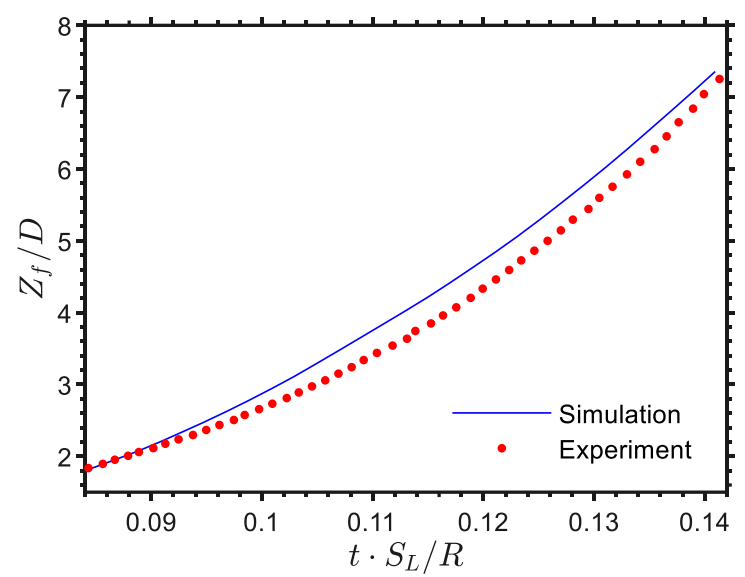

Figure 3.8: Flame tip evolution in a channel of width $0.75 \mathrm{~mm}$ [19] (experiment vs simulations).

\subsubsection{Obstructed Channel}

In this dissertation, validation is carried out for obstacle version of the code, based on the experiment of Sahoo [19]. This experiment was conducted for stochiometric methane/air burning in a channel of $2 \mathrm{~m}$ long, with a square cross section, $7.5 \mathrm{~cm} \times 7.5 \mathrm{~cm}$, and with the obstacle spacing being $1 / 16$ of the channel width and with blockage ratio of $2 / 3$. The optical access to the channel shows arrays of obstacles, see Fig. 3.7. The parameters used to simulate the experiments are the blockage ratio of $2 / 3$, channel width $24 L_{f}$, where $L_{f}=4.0 \times 10^{-5} \mathrm{~m}, S_{L}=0.364 \mathrm{~m} / \mathrm{s}$ and $\Theta=8$. The result of the simulation shows good predictions at the early stage of the propagation when compared against the experiment as seen in Fig. 3.8.

\subsection{Details of the parametric study}

The results of the parametric study conducted in this dissertation work are presented in the next few chapters. The fuel mixture initial temperature, $T_{f}=300 \mathrm{~K}$, pressure, $P_{f}=1 \mathrm{bar}$, and density, $\rho_{f}=1.16 \mathrm{~kg} / \mathrm{m}^{3}$. The walls are assumed adiabatic, $\boldsymbol{n} \cdot \nabla T=0$, and either free-slip, $\boldsymbol{n} \cdot \boldsymbol{u}=\mathbf{0}$, or non-slip, $\boldsymbol{u}=\mathbf{0}$, surfaces of the obstacles and of the channel wall, where $\boldsymbol{n}$ is a normal vector at a surface. The absorbing (non-reflecting) boundary conditions are adopted at the open end to prevent the reflection of the sound waves and weak shocks. The thermal expansion ratio ranges between $\Theta=5 \sim 10$, the Lewis number is in the range $0.2 \leq L e \leq 2.0$ and the molecular weight $M=$ $29 \mathrm{~kg} / \mathrm{kmol}$. The initial Mach number associated with flame propagation is $M a_{0}=S_{L} / c_{0}=$ $10^{-3}-10^{-2}$. Geometry is described by $\operatorname{Re}=R S_{L} / v=R / \operatorname{Pr} L_{f}=R / L_{f}=12,24,36,48 ; \Delta Z /$ $R=1 / 4,1 / 2,2 ; \alpha=1 / 3,1 / 2,2 / 3$. The computational grid consists of square cells of size 
$0.2 L_{f} \times 0.2 L_{f}$; this resolution has been extensively tested for convergence in Ref. [16]. This grid also shows good predictions for non-equidiffusive flames as shown in Fig. 3.9. For a flame in a channel with both ends open, the initial parameters of the fuel mixture $\rho=\rho_{f}, T=T_{f}, u_{z}=-S_{L}$, while those at the burnt matter were $\rho=\rho_{\mathrm{f}} / \Theta, T=\Theta T_{f}, u_{z}=-\Theta S_{L}$.

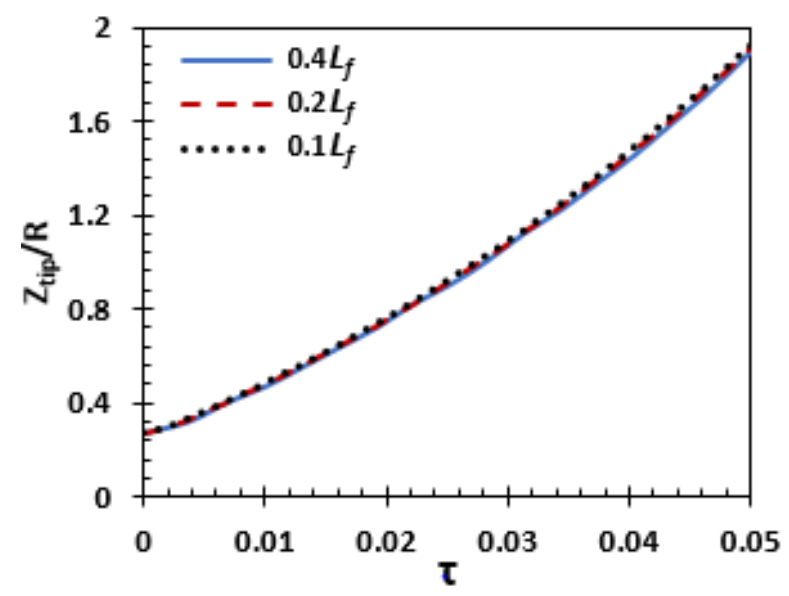

Figure 3.9: Resolution test: The scaled flame tip position versus the scaled time for $\alpha=1 / 3, L e=0.2$ and various square mesh sizes.

\section{Flame Acceleration in Semi-Open Cylindrical Channels}

The cases of free-slip and non-slip boundary conditions with various $R, \Delta Z$, and $\alpha$ are compared. A reasonable way to measure a relative deviation between the free-slip and no-slip conditions results is calculating the following quantity (in \%):

$$
E=\left|\left(Z_{f, \text { slip }}-Z_{f, n o-s l i p}\right) / Z_{f, n o-s l i p}\right| \times 100 .
$$

\subsection{Effects of the Wall Surface on Flame Acceleration: $R / 4 \leq \Delta Z \leq R / 2$}

The scaled flame tip position $Z_{f} / R$ versus the scaled time $\tau=t S_{L} / R$ is presented in Figs. $4.1-$ 4.3, with the free-slip and non-slip boundary conditions shown by the solid black and the dashed red lines, respectively, in all the plots. It is seen that the effect of free-slip and no-slip surface boundary conditions is minor if the obstacles spacing is small, $\Delta Z \leq R / 2$, and this is true for all $\alpha$ considered. Indeed, both the curves almost coincide in the cases considered. 


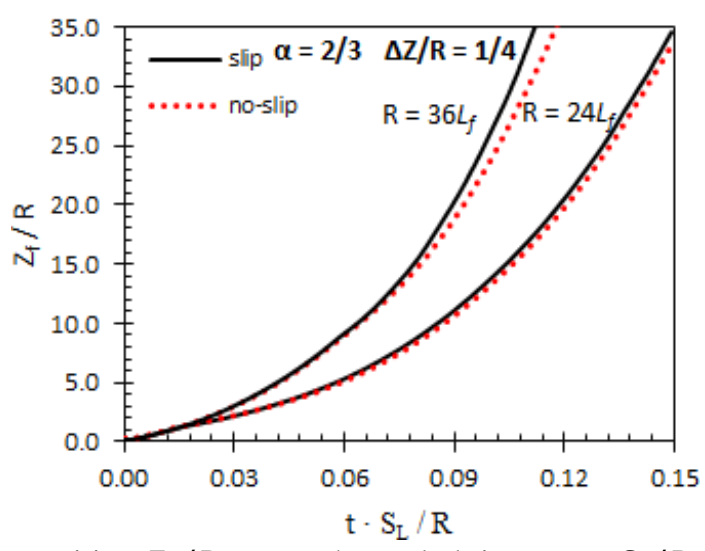

Figure 4.1: The scaled flame tip position $Z_{f} / R$ versus the scaled time $\tau=t S_{L} / R$ for $\alpha=2 / 3, \Delta Z / R=1 / 4$, and various $R=24 L_{f}$ and $36 L_{f}$.

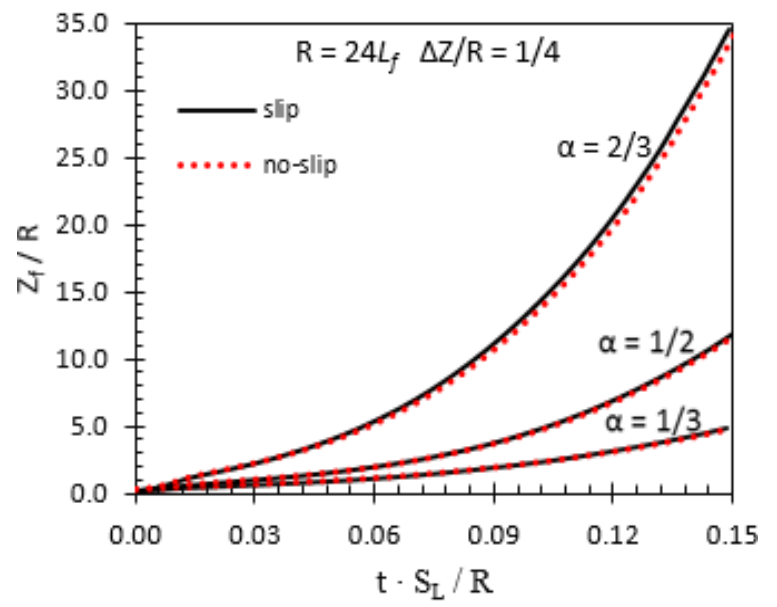

Figure 4.2: The scaled flame tip position $Z_{f} / R$ versus the scaled time $\tau=t S_{L} / R$ for $R=24 L_{f}, \Delta Z / R=1 / 4$, and various $\alpha=1 / 3,1 / 2$, and $2 / 3$.

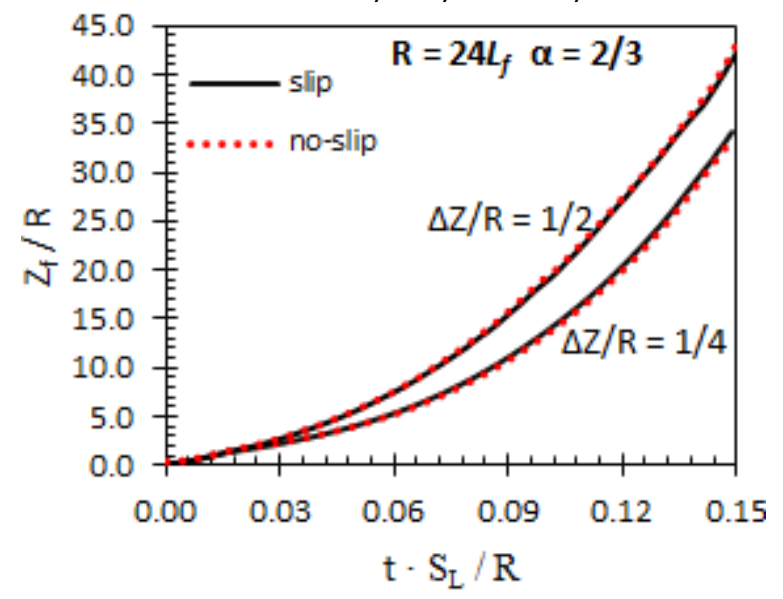

Figure 4.3: The scaled flame tip position $Z_{f} / R$ versus the scaled time $\tau=t S_{L} / R$ for $R=24 L_{f}, \alpha=2 / 3$ and various $\Delta Z / R=1 / 4$ and $1 / 2$. 


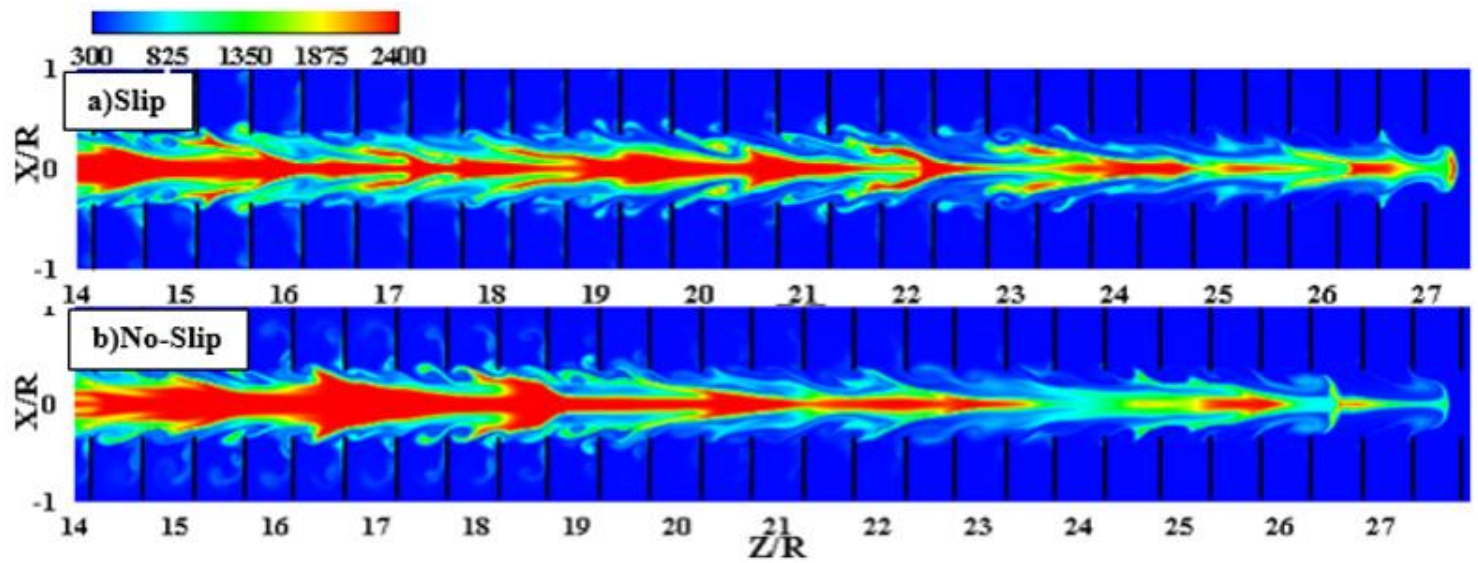

Figure 4.4: The color temperature snapshots [in K] for burning in an obstructed tube with free-slip (a) and non-slip (b) walls for $R=24 L_{f}, \alpha=2 / 3, \Delta Z=R / 2$, and taken at the same time instant $\tau=t S_{L} / R=0.15$ in both cases.

The difference calculated from Eq. (4.1) does not exceed 1 4 \% for all the cases depicted in Figs. $4.1-4.3$; wall shear stress slightly moderates flame acceleration for $\Delta \mathrm{Z}=\mathrm{R} / 4$, and very slightly promotes it for $\Delta \mathrm{Z}=\mathrm{R} / 4$. This result certifies a minor impact of the free-slip/no-slip boundary conditions and thereby justifies the Bychkov model of flame acceleration in obstructed channels, which employs the free-slip obstacles and walls. The similarity of the colorful snapshots of Fig. 4.4 taken at time instance $\tau=0.15$ also drives the same conclusion. This result can be explained by the fact that the flow is mainly driven in the axial direction such that the small obstacle spacing mitigates a potential effect of wall shear stress (if any). In fact, Ref. [18] suggested the same conclusion with the same explanation when studying the thermal boundary conditions at the walls and obstacles in obstructed channels.

\subsection{Effects of the Wall Surface Friction on Flame Acceleration: $R / 2 \leq \Delta Z \leq R$}

The effects of wall shear stress in wider obstacle spacing are also investigated and the results obtained are plotted in Figs. 4.5 - 4.7. It is observed that the results for both slip and non-slip surfaces practically coincide for a small $\Delta Z, \Delta Z \leq R / 2$, and as discussed previously, the difference between them is observed when $\Delta Z=R$. 


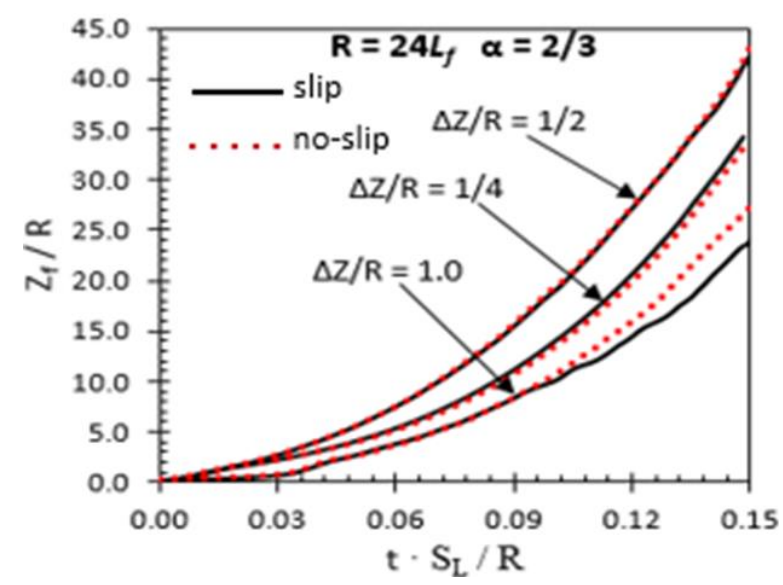

Figure 4.5: The scaled flame tip position $Z_{f} / R$ versus the scaled time $\tau=t S_{L} / R$ for $R=24 L_{f}, \alpha=2 / 3$ and various $\Delta Z / R=1 / 4,1 / 2$, and 1 .

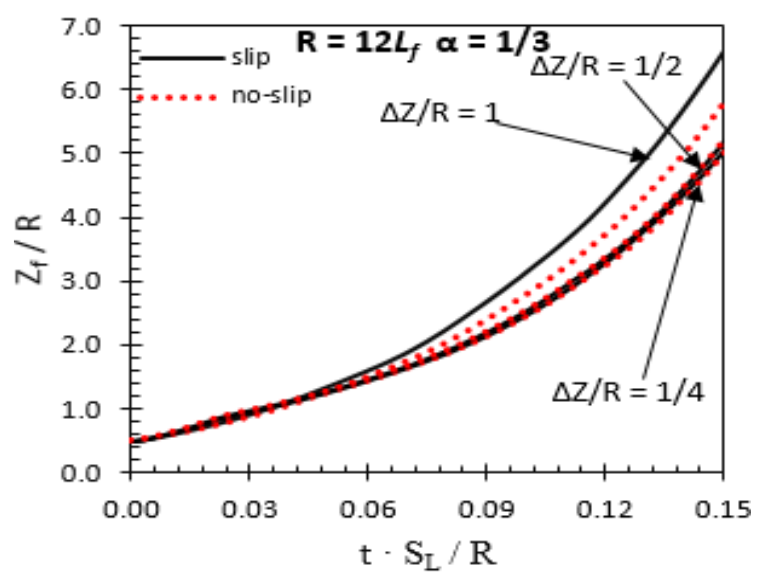

Figure 4.6: The scaled flame tip position $Z_{f} / R$ versus the scaled time $\tau=t S_{L} / R$ for $R=12 L_{f}, \alpha=1 / 3$ and various $\Delta Z / R=1 / 4,1 / 2$, and 1.

Additionally, the color snapshots are taken at time instance of $\tau=0.225$ from the simulations for the slip and non-slip boundary conditions, as presented in Figs. 4.8-4.10. It is seen that there is a minor change between the slip and non-slip walls, with the flame tip being ahead by about $1.5 R$ for the no-slip case. This result can potentially be attributed to the fact that the vortices in the pockets are barely in contact with the walls. 


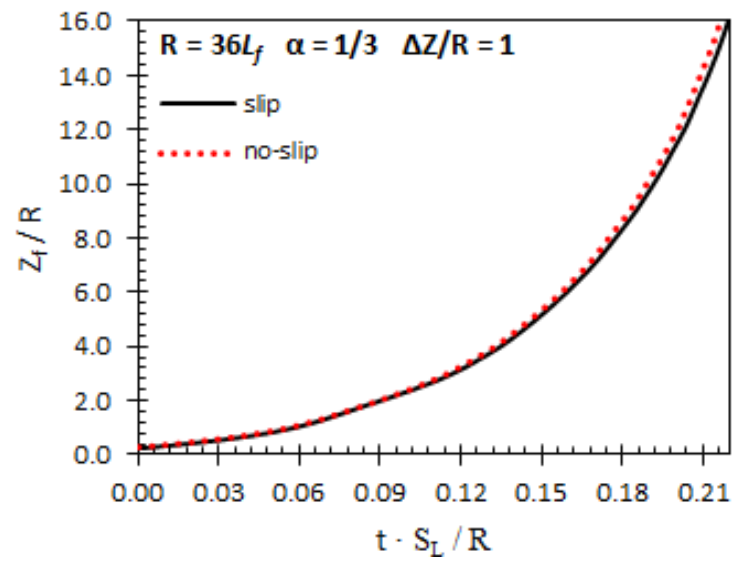

Figure 4.7: The scaled flame tip position $Z_{f} / R$ vs the scaled time $\tau=t S_{L} / R$ for $R=36 L_{f}, \alpha=1 / 3, \Delta Z / R=1$.

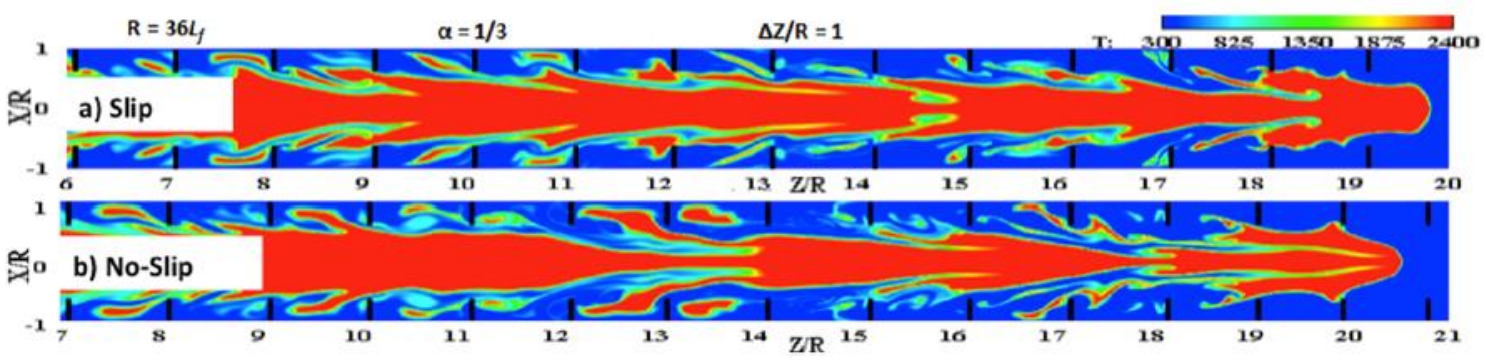

Figure 4.8: The color temperature snapshots [in K] for burning in an obstructed tube with free-slip (a) and non-slip (b) walls for $R=36 L_{f}, \alpha=1 / 3, \Delta Z / R=1$ and taken at the same time instant $\tau=t S_{L} / R=0.225$ in both cases.

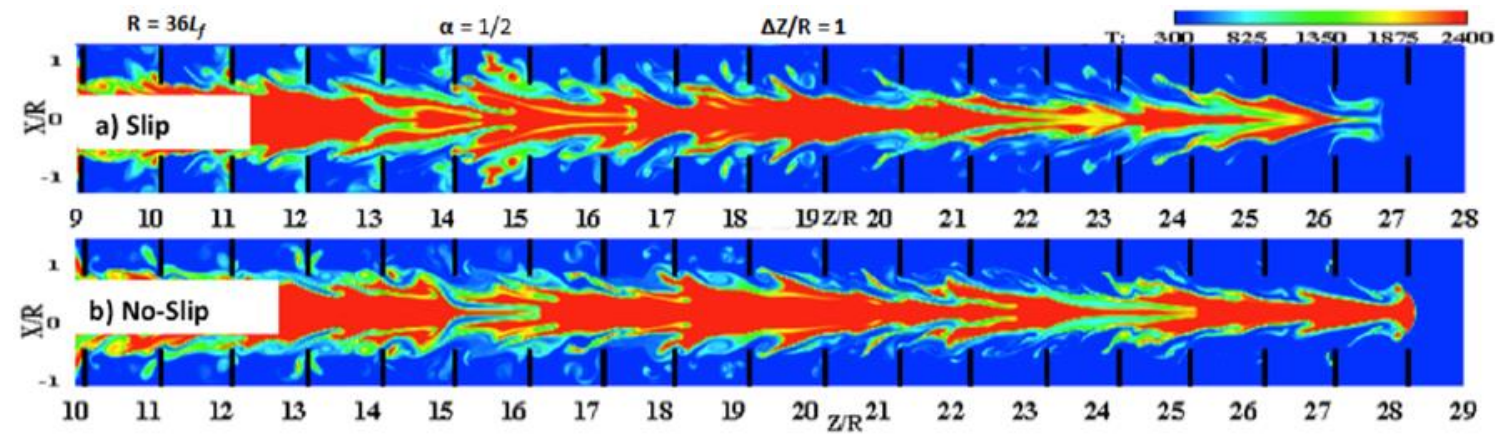

Figure 4.9: The color temperature snapshots [in K] for burning in an obstructed tube with free-slip (a) and non-slip (b) walls for $R=36 L_{f}, \alpha=1 / 2, \Delta Z / R=1$ and taken at the same time instant $\tau=t S_{L} / R=0.225$ in both cases. 


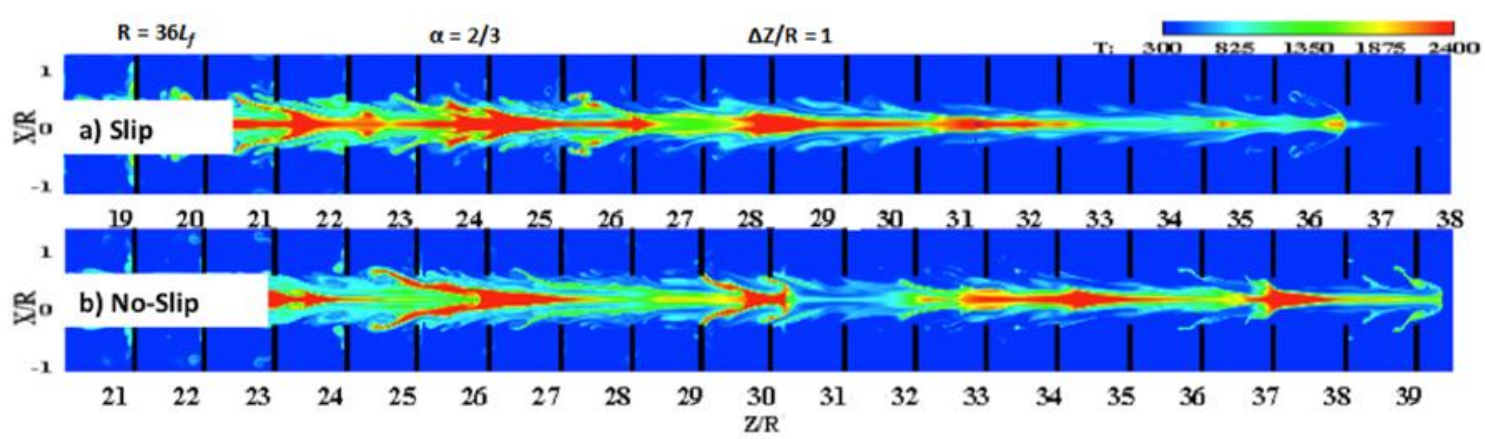

Figure 4.10: The color temperature snapshots [in K] for burning in an obstructed tube with free-slip (a) and non-slip (b) walls for $R=36 L_{f}, \alpha=2 / 3, \Delta Z / R=1$ and taken at the same time instant $\tau=t S_{L} / R=0.225$ in both cases.

\subsection{Effects of the Wall Surface of Flame Acceleration: $\Delta Z=2 R$}

For the widest spacing considered, $\Delta Z=2 R$, a remarkable difference between the slip and non-slip surfaces was observed, as shown in Figs. 4.11 - 4.14. Here, wall friction promotes acceleration, substantially, as compared to the free-slip conditions. Based on Eq. (4.1), the difference in flame tip position is as large as $24 \%$ in that case. Such a discrepancy can be attributed to the formation of high vorticity in the pockets between the obstacles in this configuration, due to wall shear stress and the obstacles. Obviously, vorticity evolves differently with slip and non-slip walls, with a stronger flow distortion in the latter case, leading thereby to faster flame acceleration. It is recalled, in this respect, that the Bychkov model does not consider vorticity, and hence it is probably not fully applicable here. Moreover, the very approach of tightly-packed obstacles, $\Delta Z \ll$ $R$, is broken when $\Delta Z$ exceeds $R$. In fact, an inapplicability of the Bychkov formulation for $\Delta Z>$ $R$ has been shown even in the pilot studies [16-17], as well as later, in the detailed analysis [18]. Qualitatively, the same conclusions follow from the color snapshots in Figs. 4.13 - 4.14. Indeed, while a comparison between Figures $4.13 \mathrm{a}$ and $4.13 \mathrm{~b}$ was evident, with the flame positioned at $Z_{f} \sim 27 R$ in the slip case and approximately $Z_{f} \sim 46 R$ in Figs. 4.13a and $4.13 \mathrm{~b}$ respectively. This discrepancy in the flame tip position is explained by the vorticity contour of both slip and non-slip cases in Figs. 4.14a and 4.14b, respectively. 


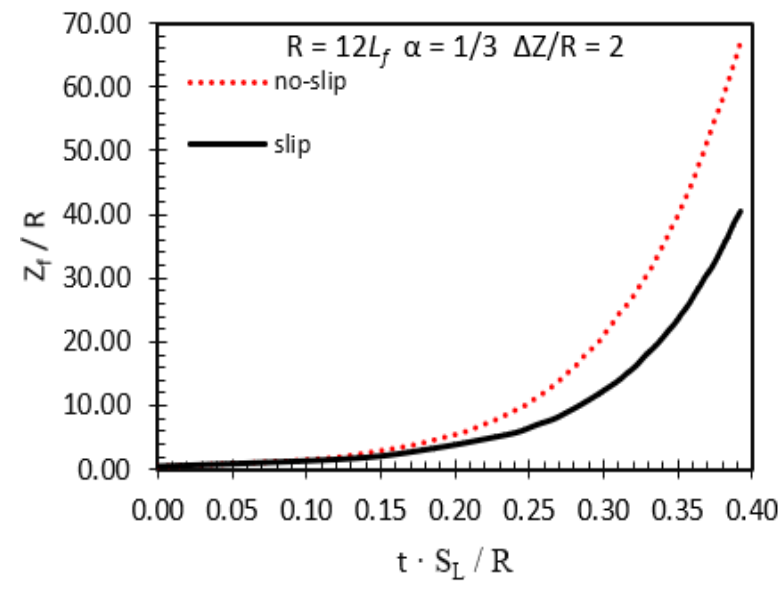

Figure 4.11: The scaled flame tip position $Z_{f} / R$ vs the scaled time $\tau=t S_{L} / R$ for $R=12 L_{f}, \alpha=1 / 3, \Delta Z / R=2$.

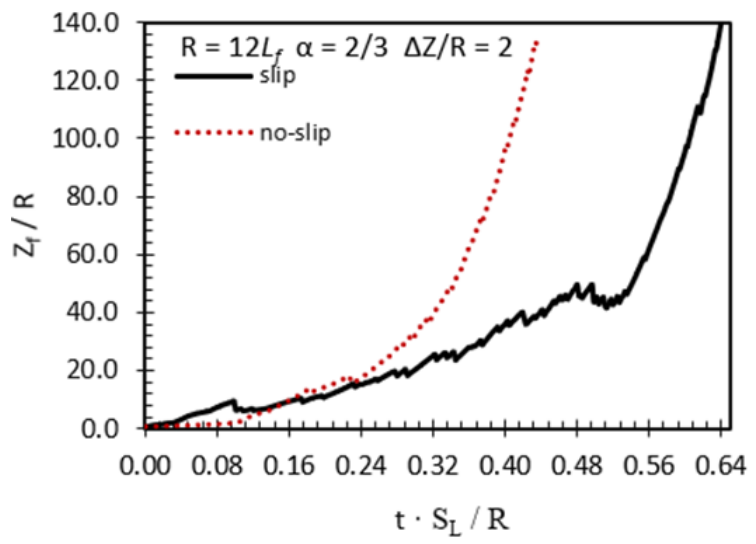

Figure 4.12: The scaled flame tip position $Z_{f} / R$ vs the scaled time $\tau=t S_{L} / R$ for $R=12 L_{f}, \alpha=2 / 3, \Delta Z / R=2$.

\section{a) Slip}

$\begin{array}{lllll}300 & 825 & 1350 & 1875 & 2400\end{array}$
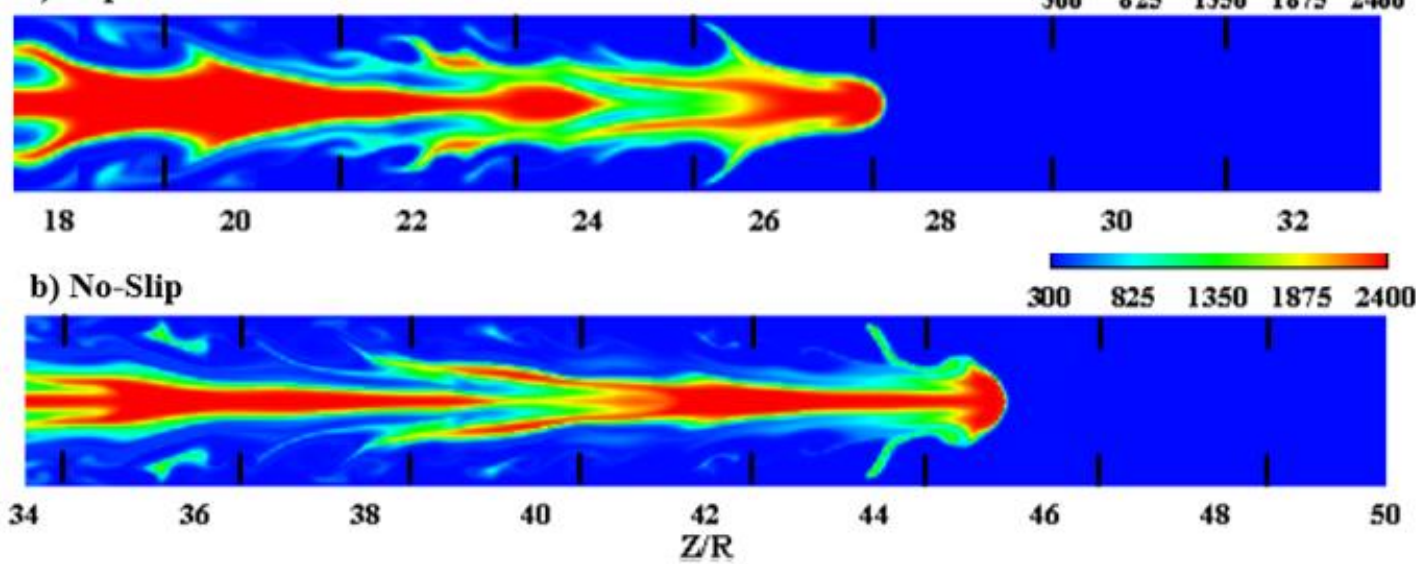

Figure 4.13: The color temperature snapshots [in K] for burning in an obstructed tube with free-slip (a) and non-slip

(b) walls for $R=12 L_{f}, \alpha=1 / 3, \Delta Z / R=2$ and taken at the same time instant $\tau=t S_{L} / R=0.36$ in both cases. 


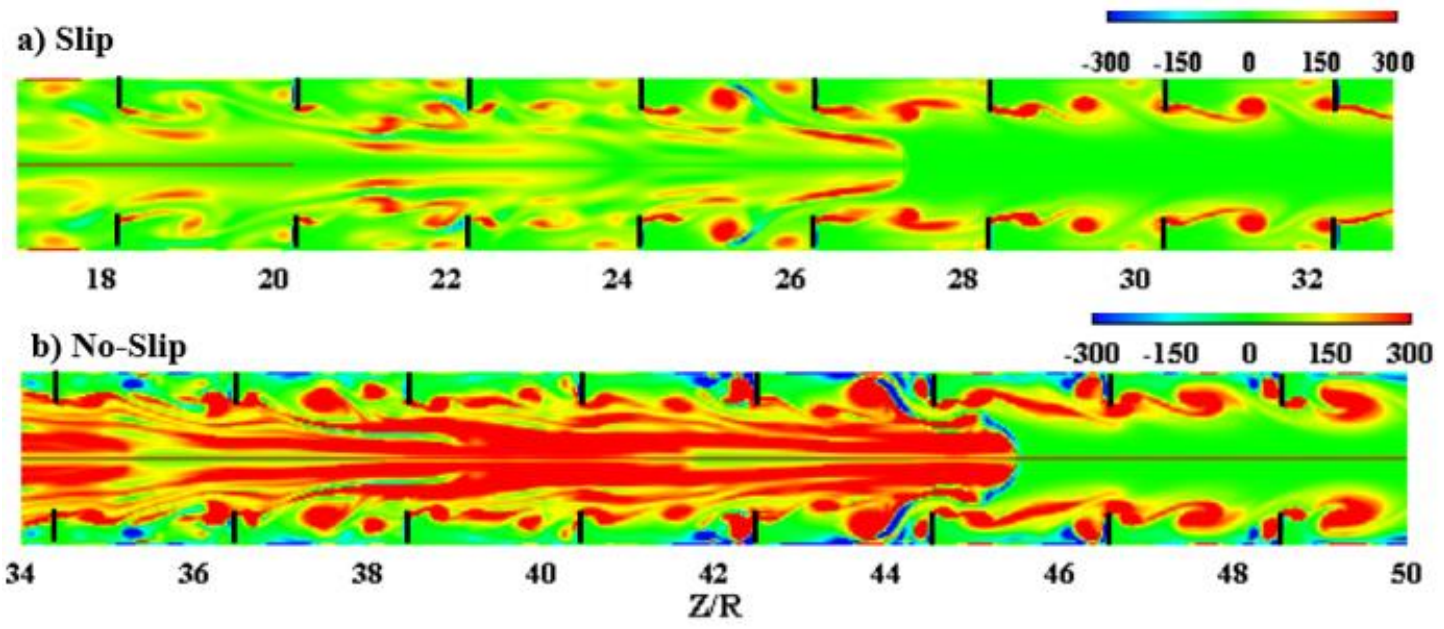

Figure 4.14: The color $\theta$-vorticity snapshots [in $\left.\sec ^{-1}\right]$ for burning in an obstructed tube with free-slip (a) and nonslip (b) walls for $R=12 L_{f}, \alpha=1 / 3, \Delta Z / R=2, \tau \equiv t S_{L} / R=0.36$. 


\section{Flame Acceleration in Semi-open Obstructed Channels}

\subsection{Morphology of Premixed Flame in Semi-open Channels}

To characterize the varying Lewis number in the obstructed channel, the flame tip Mach number

$$
M a_{t i p}(t)=U_{t i p} / c_{t i p}
$$

is introduced, with the local, instantaneous speed of sound

$$
c_{\text {tip }}=\sqrt{\left(c_{p} / c_{v}\right) \times\left(R_{p} / M\right) \times T_{t i p}}
$$

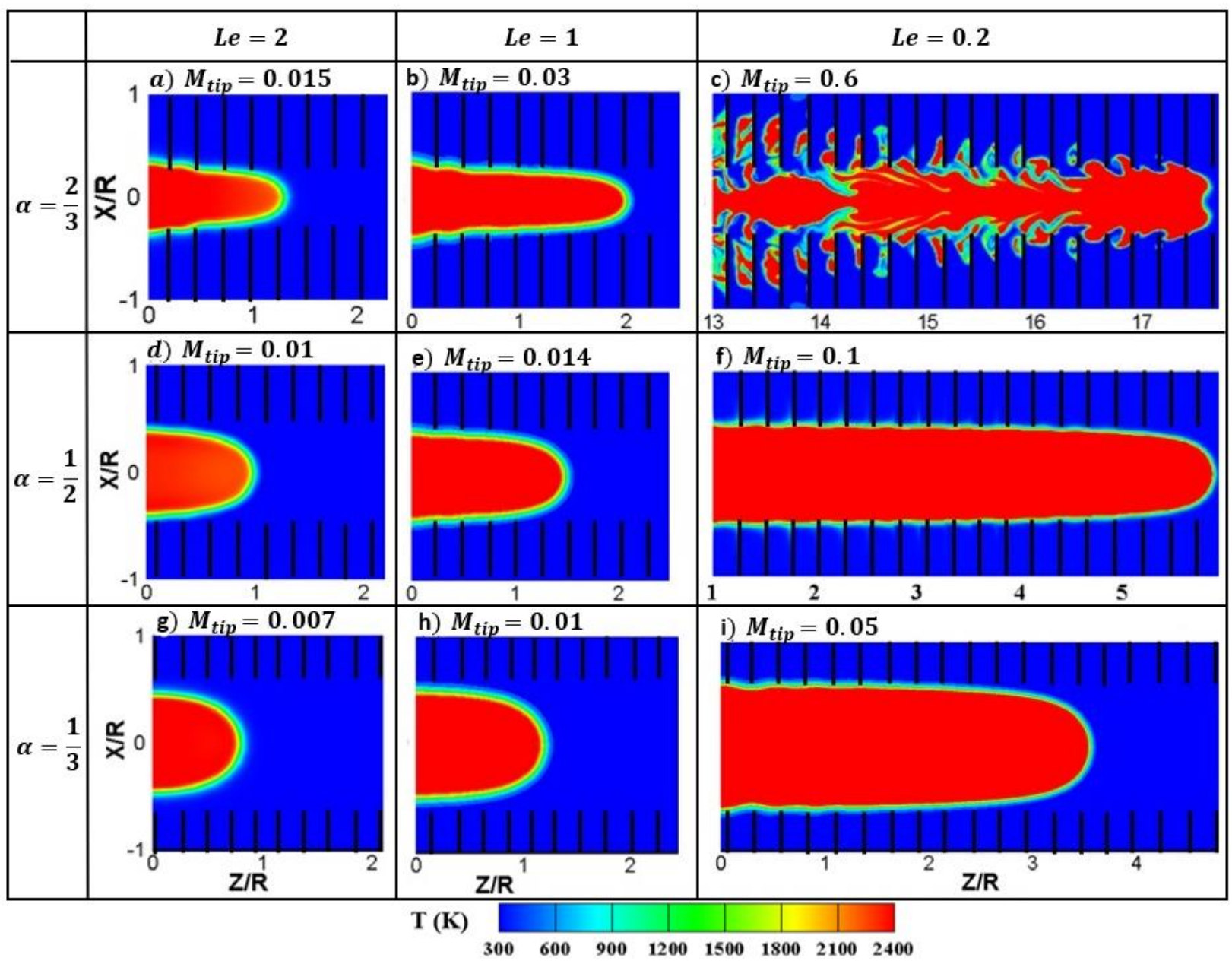

Figure 5.1: Temperature snapshots taken at the same scaled time instant.

The respective flame shapes at $\tau=0.075$ are all depicted in Fig. 5.1. The flames are represented by the temperature snapshots, with a temperature ranging from $300 \mathrm{~K}$ in the fuel till $2400 \mathrm{~K}$ in the burned matter. It is seen that the role of the Lewis number is paramount and as strong as that of the blockage ratio. Indeed, when both the effect of large $\alpha$ and non-equidiffusivity (with $L e<$ 
1) work together, as in Fig. 5.1c for $\alpha=2 / 3$ and $L e=0.2$, then the flame front is drastically folded; having propagated over considerable distance, with the flame tip Mach number as high as $M a_{\text {tip }}=0.6$. In contrast, a $L e>1$ flame in a channel with small blockage ratio accelerates very slowly, as observed in Fig. 5.1g for $\alpha=1 / 3$ and $L e=2.0$. In other cases, in Fig. 5.1, the effects of $\alpha$ and $L e$ on the flame acceleration competes, such that, almost equivalent flame structures and $M a_{t i p}$ are observed in the pairs of Figs. 5.1d and 5.1h; 5.1a and 5.1e; and even 5.1d and 5.1i.

\subsection{Quantitative Effect of the Lewis number on Flame Acceleration}
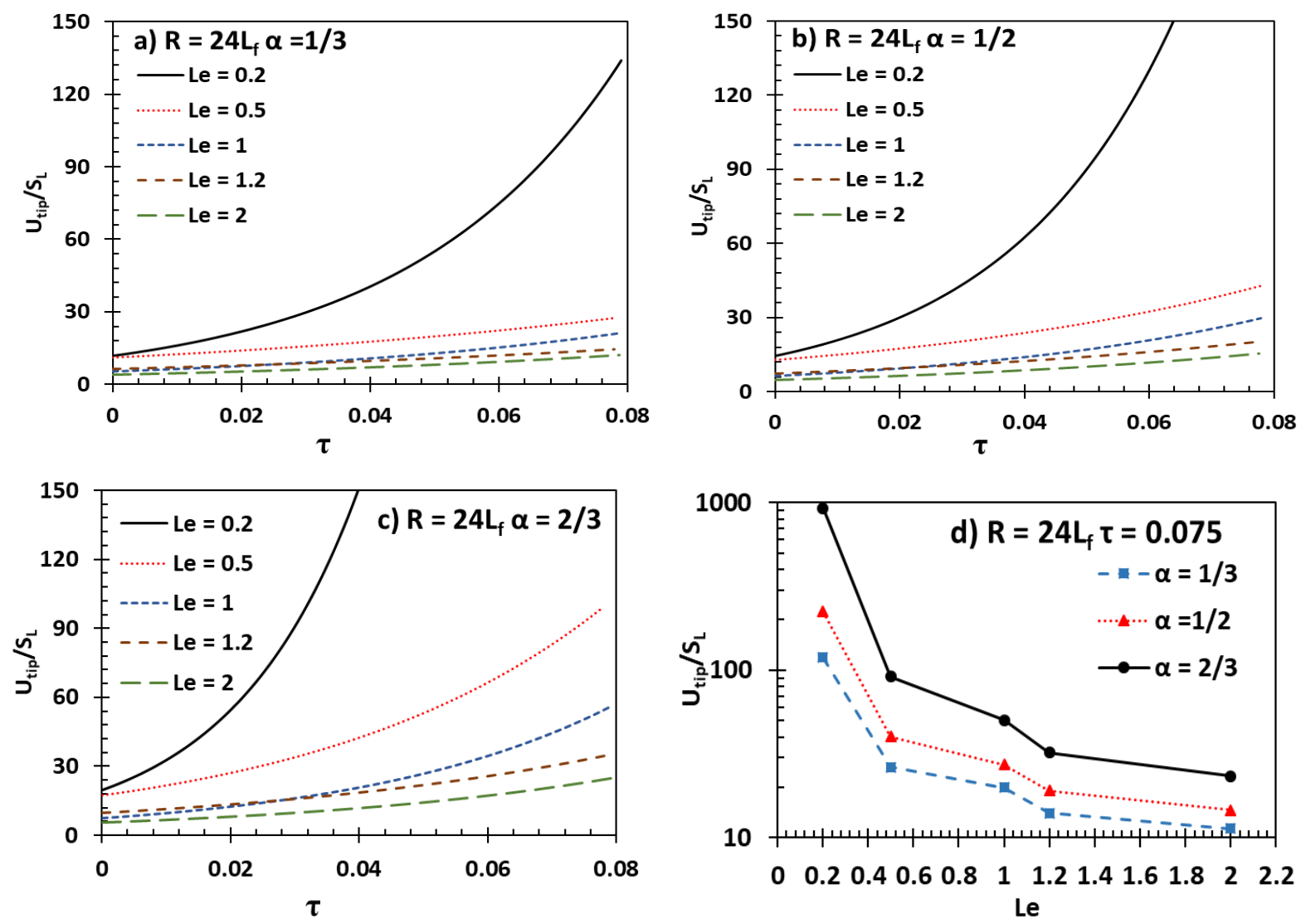

Figure 5.2: The scaled flame tip velocity $U_{\text {tip }} / S_{L}$ vs the scaled time $\tau$ for $R / L_{f}=24$ and $\alpha=1 / 3$ (a), $1 / 2$ (b), $2 / 3$

(c). Figure (d) shows the scaled flame tip velocity $U_{\text {tip }} / S_{L}$ at $\tau=0.075$ versus $L e$ for different values of $\alpha=$ $1 / 3,1 / 2,2 / 3$.

The time evolution of the scaled flame tip velocity, $U_{t i p} / S_{L}$, is presented to quantify the impact of $L e$ in Fig. 5.2. It is seen that the effect of $L e$ is very strong, especially for the $L e<1$ flames. Indeed, in all three Figs. 5.2 (a-c), $L e=0.2$ leads to an increase in $U_{t i p}$ almost by an order of magnitude as compared to the equidiffusive case, $L e=1$. The effect of $L e>1$ is substantially 
weaker, but $L e=2.0$, nevertheless, noticeably moderates FA as compared to the $L e=1$ cases. Figure 5.2 (d) depicts the variation of the logarithm of scaled tip velocity with $L e$ at $R / L_{f}=24$ and scaled time instant $\tau=0.075$ (similar to Fig. 5.1). It is shown that the flame velocity increases as $L e$ decreases for all values of $\alpha$, and greatest increase occur for the largest $\alpha$.

\subsection{Effect of Blockage Ratio on Flame Acceleration}

Figure 5.3 illustrates the role of the blockage ratio $\alpha$ in the acceleration of the flame tip. It is seen that $\alpha$-dependence is significant and much stronger than $R e$-dependence for all $L e$ considered. At the same time, the impact of $L e$ on $\alpha$-dependence is smaller than that on Re-dependence: $\alpha$ dependence does not change sign due to $L e$, but there is a noticeable quantitative effect: $\alpha$ dependence is stronger for $L e<1$ flame. For different values of $L e$, the flames exhibit slower accelerations in the unobstructed channel, $\alpha=0$, relative to the acceleration in obstructed ones.
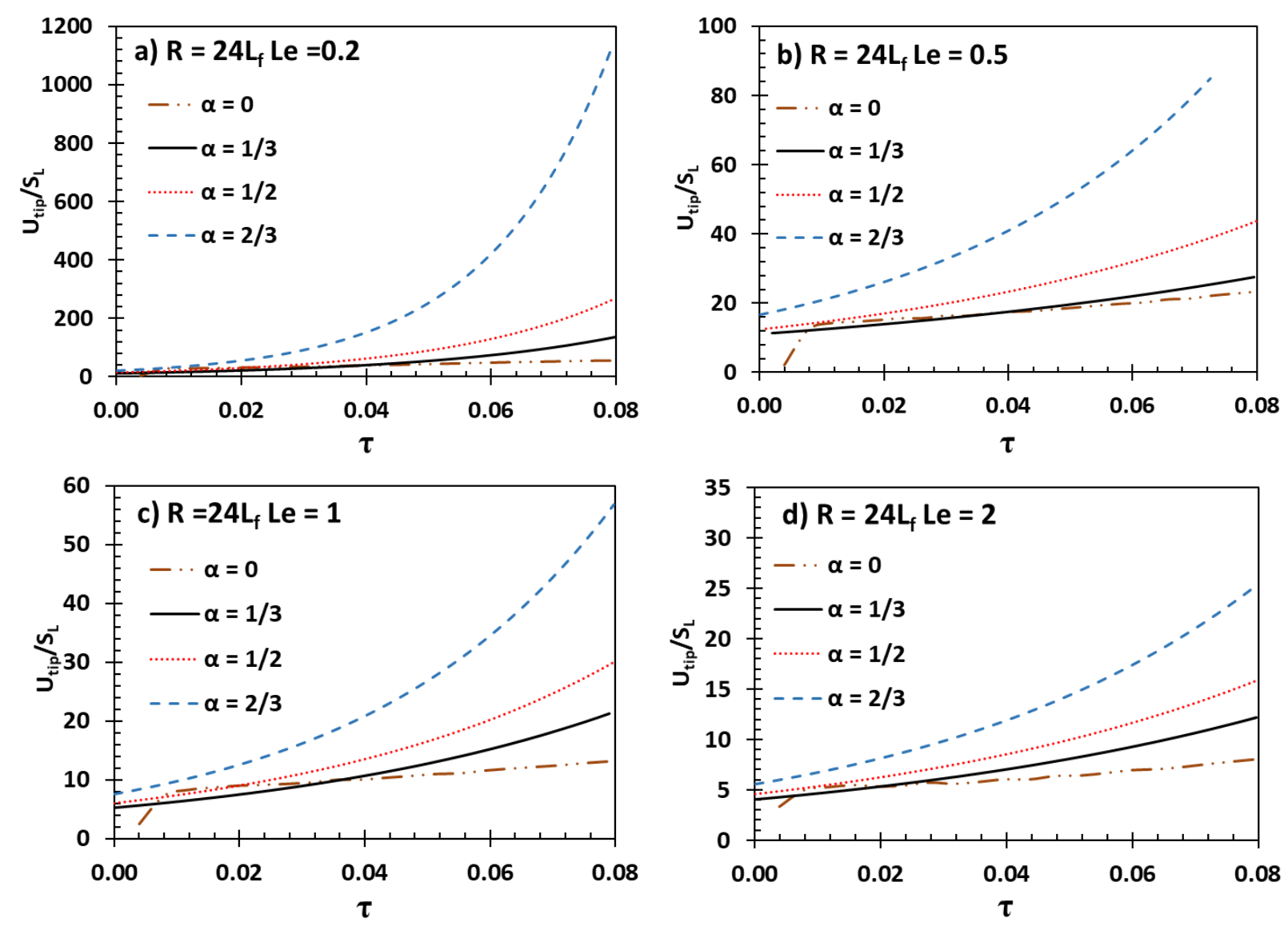

Figure 5.3: The scaled flame tip velocity $U_{\text {tip }} / S_{L}$ versus the scaled time $\tau$ for $R / L_{f}=24$ and various $L e=0.2$ (a), 0.5 (b), 1.0 (c), 2.0 (d). 


\subsection{Effect of Channel Width on Flame Acceleration}

Figure 5.4 scrutinizes the role of the channel width for various $L e$ and $\alpha$. It is seen that the impact of $R e$ is minor as all the curves for $R / L_{f}=24,36,48$ as seen in Figures 5.4 (a-d) attain very similar acceleration rates. This supports the Bychkov formulation [15] predicting $R e$-independent FA. On the other hand, Fig. 5.4 shows a very intriguing result: the impact of the Lewis number modifies the $R e$-dependence up to the opposite one. Indeed, FA weakens with $R e$ for $L e \leq 1$ flames due to decreasing flame stretch, Figs. 5.4 (a, b, c), but it is promoted with $R e$ in the $L e>1$ case for opposite reason, Fig. 5.4d. Potentially, there exist a certain Le threshold that would correspond to the change of the trend and thus provide the complete $R e$-independence. While adiabatic walls are assumed here, it is however important to note that presence of heat losses at the walls may modify the flow behind the flame front, and thus, changing the dynamics of the flame tip.
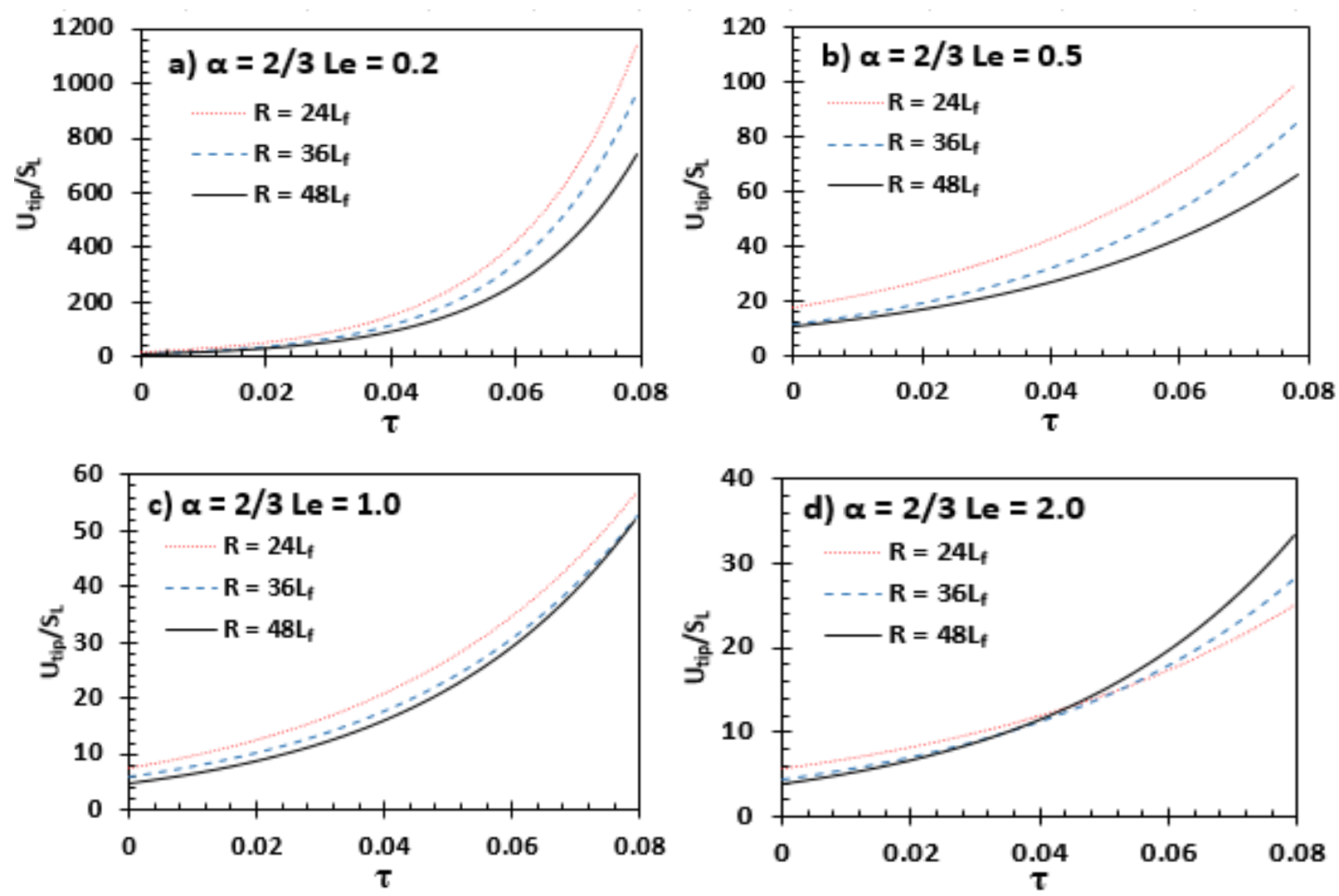

Figure 5.4: The scaled flame tip velocity $U_{\text {tip }} / S_{L}$ versus the scaled time $\tau$ for $\alpha=2 / 3$ and various $L e=0.2$ (a), 0.5 (b), 1.0 (c), 2.0 (d). 

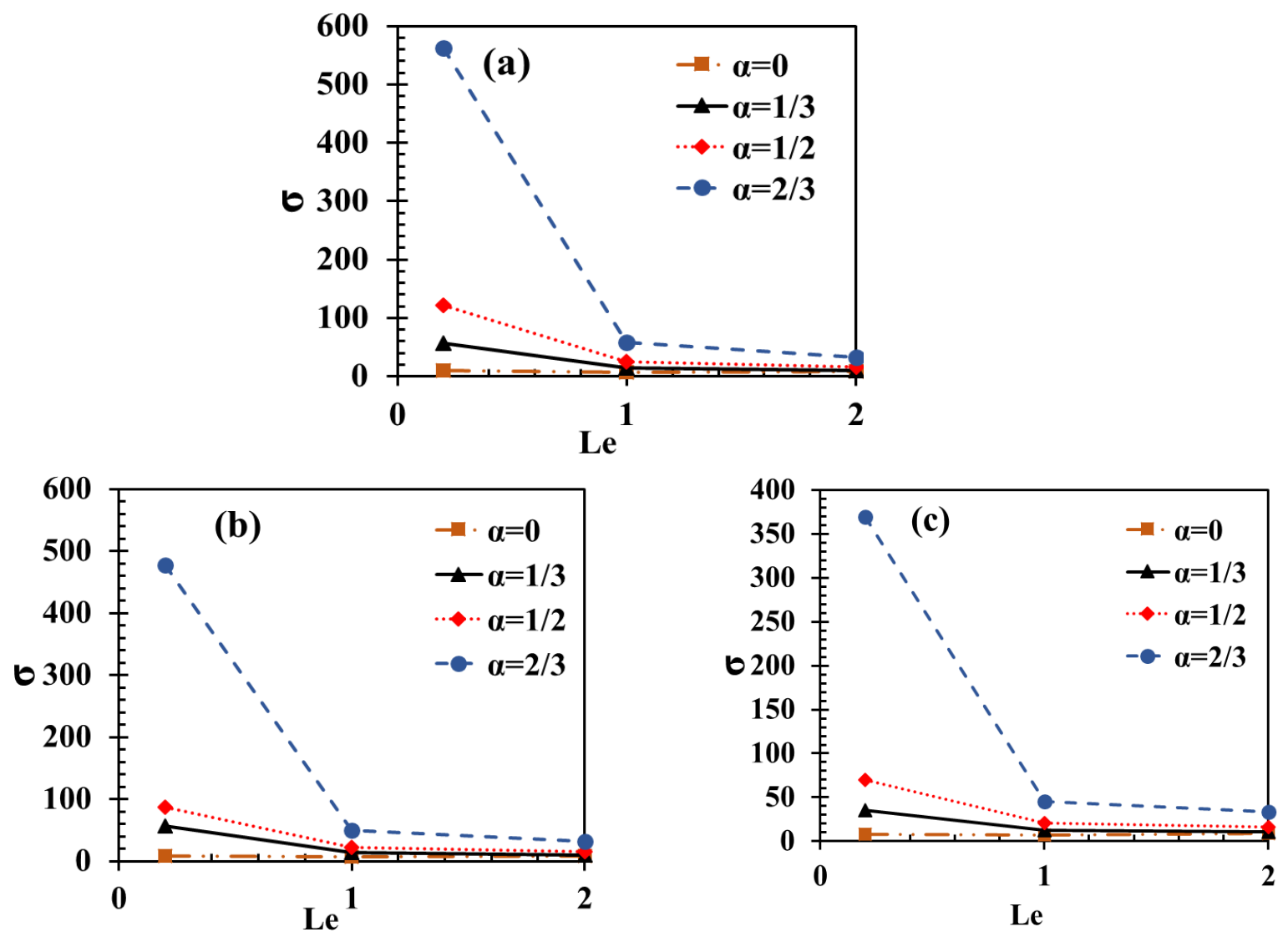

Figure 5.5: The exponential acceleration rate $\sigma$ versus the Lewis number $L e$ for $R / L_{f}=24$ (a), 36 (b), and 48 (c), with $\alpha=1 / 3,1 / 2$, and $2 / 3$ in each figrure.

Finally, the acceleration trends were analyzed, and the cases with the exponential trends are exhibited. The exponential acceleration rate $\sigma$ is related to flame tip position by $Z_{t i p} \approx e^{\sigma \tau}$ and was estimated as the slope from the plot of scaled flame tip position $Z_{t i p}$ versus scaled time $\tau$. The resulting $\sigma$ versus $L e$ is plotted in Fig. 5.5, for $R e=24,36$ and 48, respectively, with $\alpha=$ $1 / 3,1 / 2$ and $2 / 3$ in each figure. The acceleration rate $\sigma$ is the largest for non-equidiffusive cases of $L e<1$. Also, in order to unify the analysis, similar to Bychkov et al. [15], the combination $\sigma Z_{f} / \Theta R$ is plotted versus $\sigma S_{L} t / R$ in Fig. 5.6; it is recalled that $\sigma=(\Theta-1) /(1-\alpha)$. While Ref. [15] was limited to equidiffusive flames, $L e=1$, with all the data collapsing into a single curve for various $L e$, and the set of curves associated with different $L e$ differs. However, for each given $L e$, the plots collapse into a single curve, consistent with the Bychkov model. 


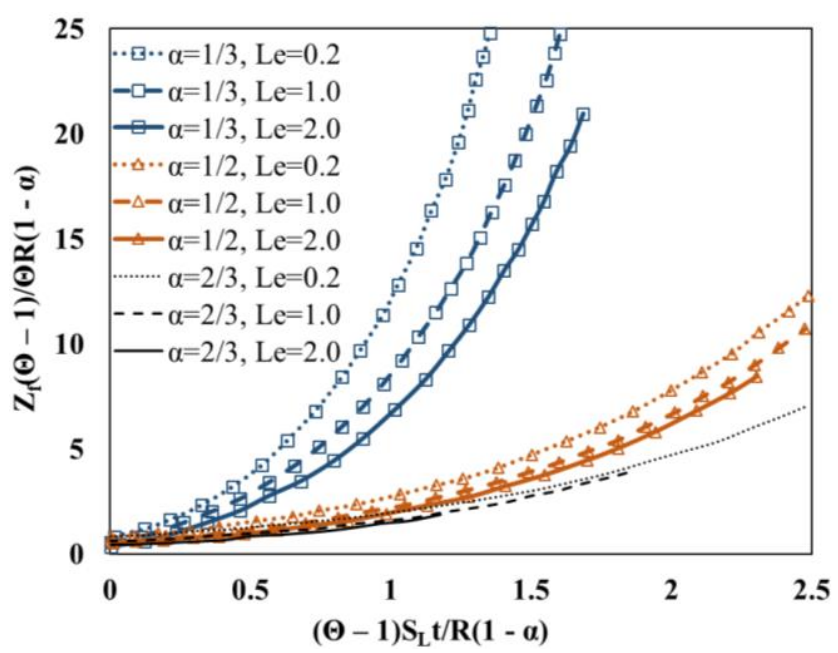

Figure 5.6: The scaled flame tip position $\sigma Z_{f} / \Theta R$ versus the scaled time $\sigma t S_{L} / R$ for various $L e=0.2$ (square markers), 1.0 (triangle markers) and 2.0 (without marker), with three different $\alpha=1 / 3$ (solid), $1 / 2$ (dashed) and 2/3 (dotted) for each given Le. 


\section{Flame Oscillation in Obstructed Channels with Both Ends Open}

\subsection{Morphology of Oscillating Premixed Flame}

Premixed flame morphology as well as flame-generated flow velocity fields in obstructed channels with both ends open are explored and presented in Fig. 6.1. An initially planar ZFK flame (not shown in the figures) promptly acquires a curved shape, it is observed that the length and curvature of the flame front vary as the flame propagates. Specifically, the variations of the scaled burning rate $U_{w} / S_{L}$ show that the flame front oscillates in a periodic manner. As a result, the flame dynamics and morphology as well as the evolution of the velocity field during the oscillation are analyzed. The color temperature snapshots of Fig. 6.1 show the flame front evolution for $\alpha=1 / 3$, Fig. 6.1a, $\alpha=1 / 2$, Fig. 6.1b, and $\alpha=2 / 3$, Fig. 6.1c, respectively. In all three cases $\Delta \mathrm{Z}=R / 4$ and $\Theta=8$ such that the temperature varies from $300 \mathrm{~K}$ in the fresh fuel mixture (blue) till $2400 \mathrm{~K}$ in the burned gas (red). To better illustrate the evolution of the flow and the flame structure in the cavities, the zoomed version of the snapshots (ii) and (iv) for $\alpha=1 / 2$ in Fig. 6.1d and 6.1e, respectively, are also presented. The flow streamlines are shown in white. The flame segment in the free (unobstructed) part of the channel generally acquires a concave shape, with a formation of a cusp directed towards the burned gas at the centerline. The other flame segments enter the pocket between the obstacles. Only the upper half of this channel was simulated as depicted in Figs. 6.1e and $6.1 \mathrm{~d}$, while $6.1 \mathrm{a}-6.1 \mathrm{c}$ represent the entire width of a channel for illustrative purposes only.

At the initial stage of each oscillation, the flame is almost quiescent as shown by the snapshots (i) - (ii) in all the figures. The duration of such a stationary stage increases with $\alpha$, whereas the concavity of the flame front is smaller for larger $\alpha$. During this stage, a flame starts consuming the fuel mixture in the pocket; the burning gas expands, and a newly generated gas volume bends a flame segment in the pocket, making it convex, while that near the centerline remains concave; see also Fig. 6.1d. Consequently, the cumulative surface area (length) of the flame front grows, thereby promoting the total burning rate and yielding FA at this stage. However, as burning proceeds in a pocket, the flame eventually enters the next pocket and its front gets broken by the obstacle; see also Fig. 6.1e. Such breaking of the flame front terminates acceleration, so the flame decelerates, as shown by snapshots (iii)-(iv) in Figs. 6.1 (a-c), until the shape (v) of Figs. 6.1a, 6.1b is acquired. This acceleration-deceleration scenario described above repeats on a regular basis resulting in flame oscillations. As a result, a flame propagating in the pockets between 
the obstacles experiences several stages of burning, during which the instantaneous flame shape, length and velocity substantially vary, thus exhibiting not a steady state but the oscillations. However, these oscillations are identical if the obstacles are identical and the pockets between them are identical.
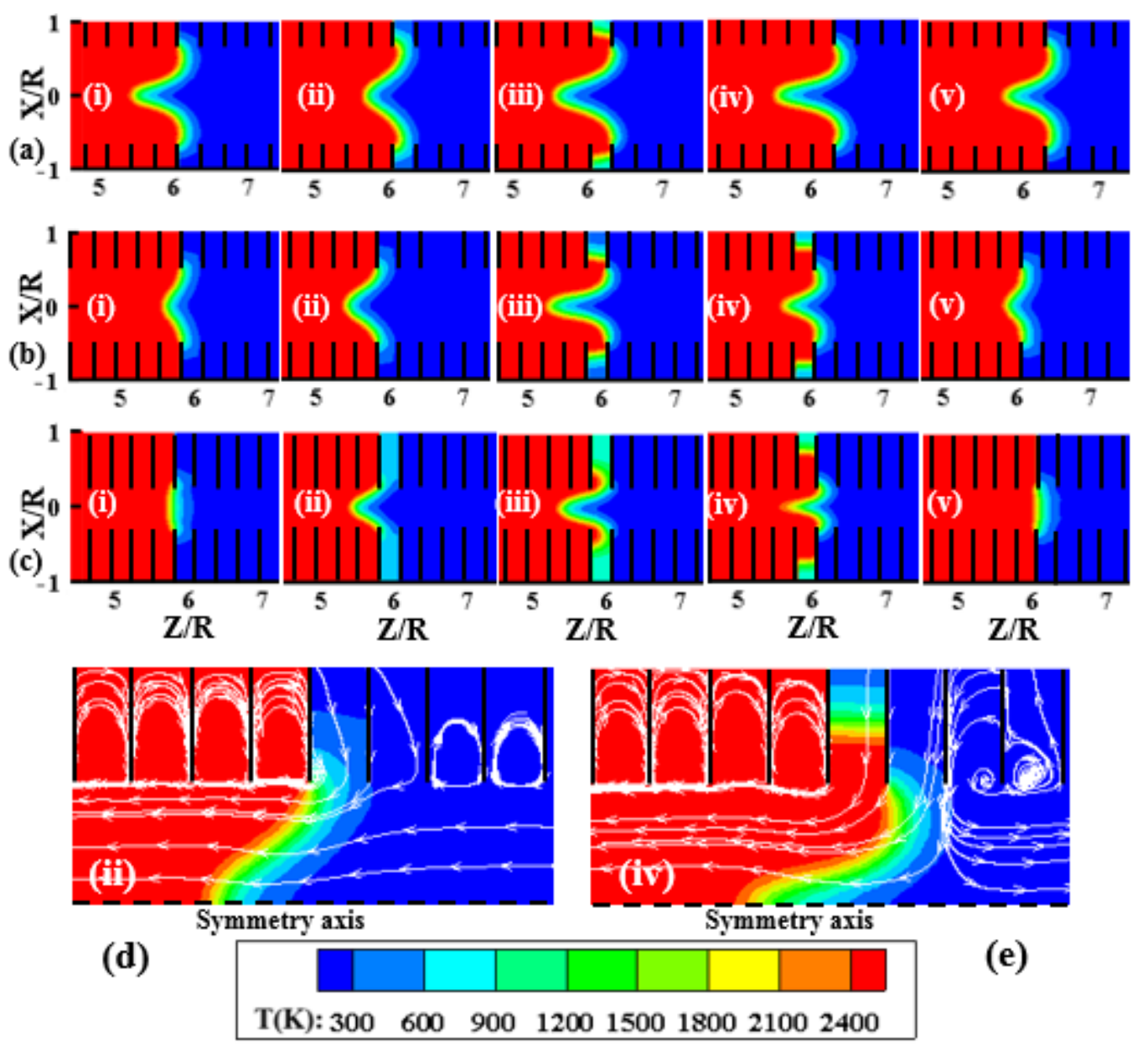

(e)

Figure 6.1: Consecutive color temperature snapshots and the flow streamlines (white) for the propagation of a flame with the thermal expansion ratio $\Theta=8$ in a channel with the obstacle spacing $\Delta \mathrm{z}=R / 4$ and various blockage ratios: $\alpha=1 / 3$ (a), $\alpha=1 / 2$ (b), and $\alpha=2 / 3$ (c). (d) and (e) are the zoomed versions of the snapshots (ii) and (iv) for $\alpha=1 / 2$.

\subsection{Impacts of Blockage Ratio on an Oscillating Premixed Flame}

Figure 6.2 scrutinizes the impact of the blockage ratio on the burning rate oscillations by comparing the cases of $\alpha=1 / 3,1 / 2,2 / 3$ (Figs. $6.2 \mathrm{a}-6.2 \mathrm{~b}$, respectively), with fixed $\Delta Z=R / 4$ and $\Theta=8$. It is seen that the oscillations are nonlinear, and this nonlinearity is stronger for larger $\alpha$. In the $\alpha=1 / 3$ channel, a secondary peak appears for each oscillation. Overall, there are three basic quantities characterizing the flame oscillations: the oscillation period $\tau_{p}$, amplitude $\Delta U$, 
which slowly dampens with time in Fig. 6.2, and the average burning rate during an oscillation $\left\langle U_{w}\right\rangle$. According to Fig. 6.2, $\tau_{p}$ increases with $\alpha$ such that the largest period, with the most profound nonlinearity is seen for $\alpha=2 / 3$. The latter effect can be attributed to the fact that the larger blockage ratios correspond to the deeper pockets and thereby longer duration of a nearquiescent stage observed.

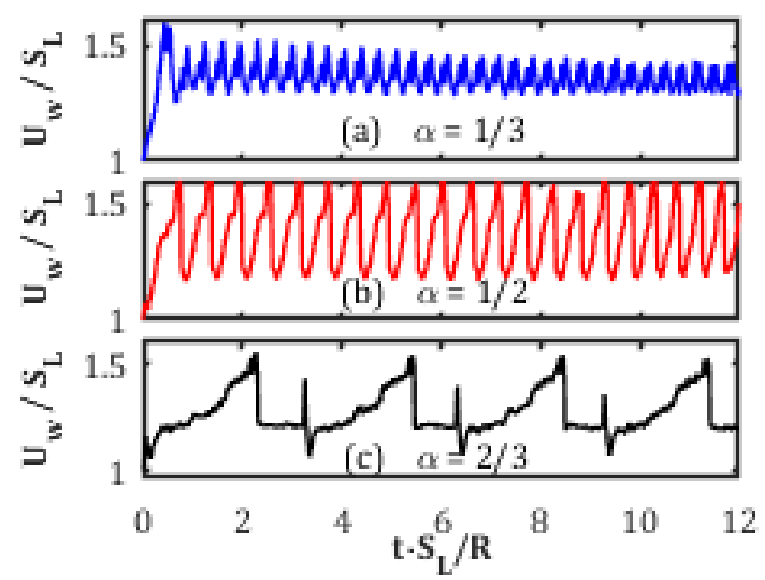

Figure 6.2: The scaled burning rate $U_{w} / S_{L}$ versus the scaled time $S_{L} t / R$ for the thermal expansion ratio $\Theta=8$, obstacle spacing $\Delta \mathrm{z}=\mathrm{R} / 4$, and various blockage ratios: $\alpha=1 / 3$ (a), $\alpha=1 / 2$ (b), and $\alpha=2 / 3$ (c).

\subsection{Impacts of Obstacle Spacing on an Oscillating Premixed Flame}

The role of $\Delta z$ is described by Fig. 6.3, where the cases of $\Delta z=R / 4$ (Fig. 6.3a), $\Delta Z=R / 2$ (Fig. 6.3b) and $\Delta z=R$ (Fig. 6.3c) are compared for fixed $\alpha=1 / 3$ and $\Theta=8$. The effect of spacing is found to be noticeable. Particularly, both the oscillation period and nonlinearity get promoted with the increase in $\Delta z / R$.
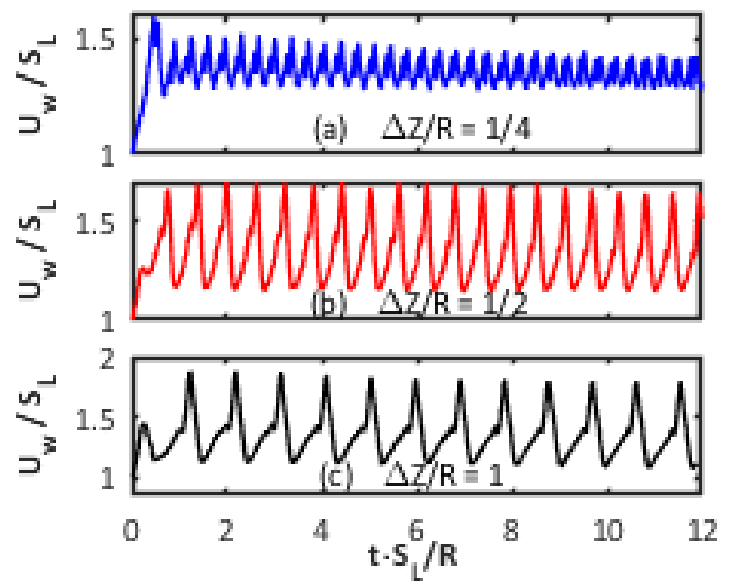

Figure 6.3: The scaled burning rate $U_{w} / S_{L}$ versus the scaled time $\mathrm{S}_{\mathrm{L}} \mathrm{t} / \mathrm{R}$ for the thermal expansion ratio $\Theta=8$, the blockage ratio $\alpha=1 / 3$ and various obstacle spacing $\Delta z=R / 4$ (a), $\Delta z=R / 2$ (b) and $\Delta z=R$ (c). 


\subsection{Impacts of the Thermal Expansion Ratio on an Oscillating Premixed Flame}

The influence of the thermal expansion of the burning matter on the flame oscillations is studied by varying the expansion ratio in the range $\Theta=5 \sim 10$. Specifically, Figure 6.4 compares the evolutions of the burning rate for $\Theta=5$ (Fig. 6.4a), $\Theta=8$ (Fig. 6.4b), and $\Theta=10$ (Fig. 6.4c) with the same $\Delta z=R / 4$ and $\alpha=1 / 3$ in all three cases. It is clearly seen that the oscillation period reduces with $\Theta$, similarly the observed nonlinearity does reduce with $\Theta$. Indeed, instead of regular and smooth oscillations for $\Theta=10$ in Fig. 6.4c, we can see strong nonlinearity and irregularity for $\Theta=5$ in Fig. 6.4a. Figures 6.5 and 6.6 are the counterparts of Fig. 6.4 for $\alpha=1 / 2$ (Fig. 6.5) and $\alpha=2 / 3$ (Fig. 6.6), respectively. In fact, all three figures yield the same conclusion: the reduction in $\Theta$ promotes both nonlinearity and the oscillation period $\tau_{p}$. At the same time, nonlinearity itself is much stronger in Figs. 6.5 and 6.6 as compared to Fig. 6.4. The latter is obviously devoted to the larger $\alpha$.

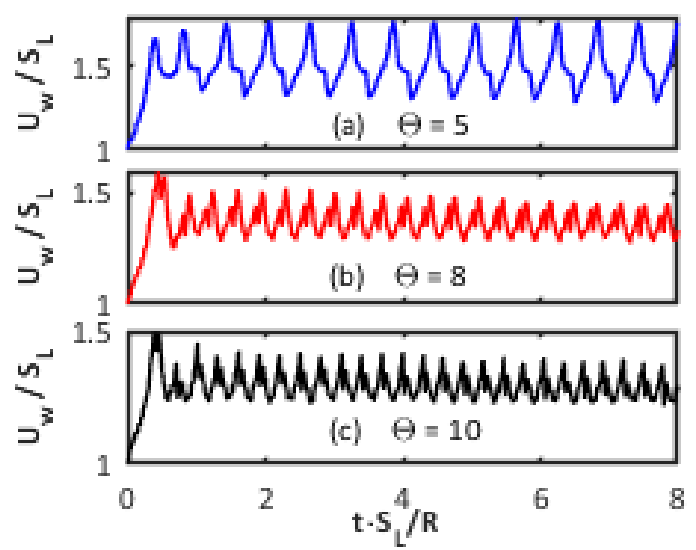

Figure 6.4: The scaled burning rate $U_{w} / S_{L}$ versus the scaled time $S_{L} t / R$ for the blockage ratio $\alpha=1 / 3$, obstacle spacing $\Delta z=R / 4$ and various thermal expansion ratios $\Theta=5$ (a), $\Theta=8$ (b), $\Theta=10$ (c).

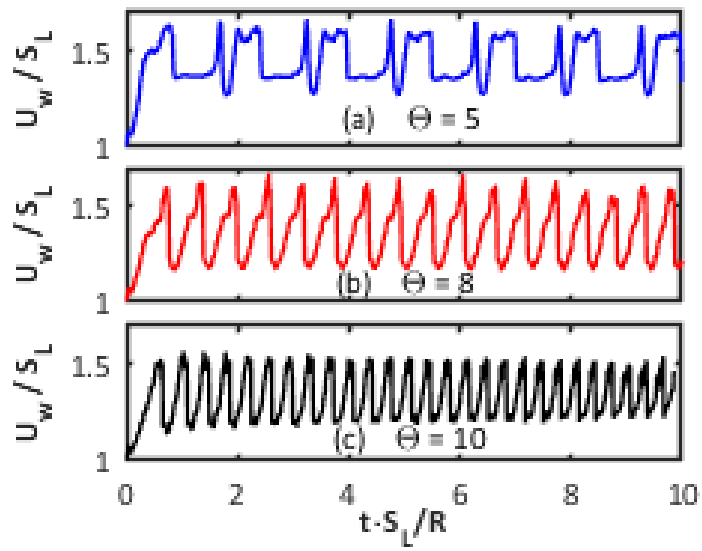

Figure 6.5: The scaled burning rate $U_{w} / S_{L}$ versus the scaled time $S_{L} t / R$ for the blockage ratio $\alpha=1 / 2$, obstacle spacing $\Delta z=R / 4$ and various thermal expansion ratios $\Theta=5$ (a), $\Theta=8$ (b), $\Theta=10$ (c). 


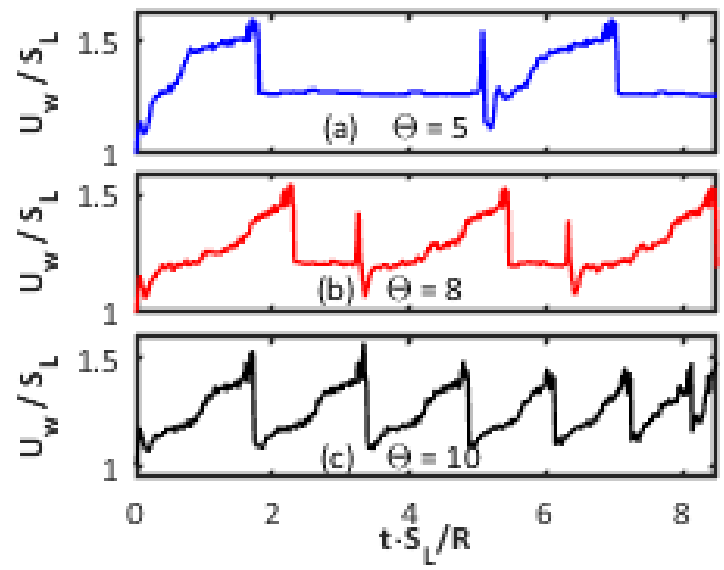

Figure 6.6: The scaled burning rate $U_{w} / S_{L}$ versus the scaled time $S_{L} t / R$ for the blockage ratio $\alpha=2 / 3$, obstacle spacing $\Delta z=R / 4$ and various thermal expansion ratios $\Theta=5$ (a), $\Theta=8$ (b), $\Theta=10$ (c).

\subsection{Unified Characteristics of an Oscillating Premixed Flame}

The scaled oscillation period $\tau_{p} S_{L} / R$, the scaled average burning rate $\left\langle U_{w}\right\rangle / S_{L}$, and the scaled oscillation amplitude $\Delta U / S_{L}$ are plotted versus the thermal expansion ratio $\Theta$, to quantify the qualitative conclusions of Figures $6.4-6.6$, Also, see Figures 6.7a - c, respectively. Specifically, Fig. 6.7a shows that $\tau_{p}$ reduces with $\Theta$ for any blockage ratio, but the effect is minor for $\alpha=1 / 3$ and it is quite moderate for $\alpha=1 / 2$. In contrast, the reduction in $\tau_{p}$ is dramatic for $\alpha=2 / 3$. The average burning rate $\left\langle U_{w}\right\rangle$, Fig. $6.7 \mathrm{~b}$, also reduces with $\Theta$ for any $\alpha$, but here the situation is qualitatively different from Fig. 6.7a: the effect appears minor for large blockage ratio, $\alpha=2 / 3$, but it is noticeable for smaller $\alpha$. As for the oscillation amplitude $\Delta U$, Fig. 6.7c, it reduces with $\Theta$ as long as the blockage ratio is moderate, $\alpha=1 / 3$, while for larger $\alpha$ the $\Theta$-dependence of $\Delta U$ is non-monotonic. 

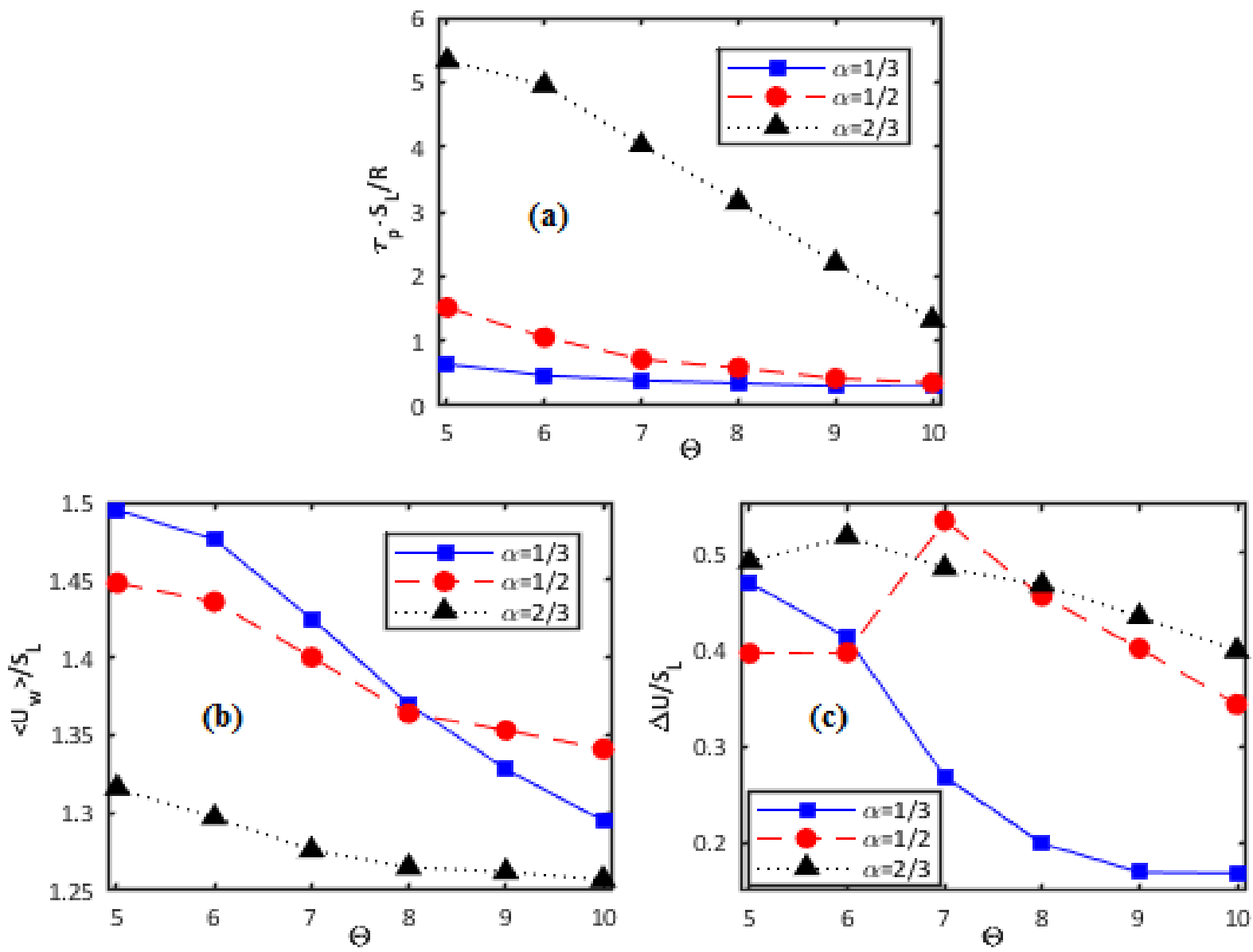

Figure 6.7: The scaled oscillation period $\tau_{p} S_{L} / R$ (a), the average burning rate during an oscillation $\left\langle U_{w}\right\rangle / S_{L}$ (b), and the scaled oscillation amplitude $\Delta U / S_{L}$ (c) versus the thermal expansion ratio $\Theta$ for the obstacles spacing $\Delta z=R / 4$ and various blockage ratios $\alpha=1 / 3,1 / 2,2 / 3$. 


\section{Flame Acceleration in Obstructed Channels with Both Ends Open}

\subsection{Morphology of the Accelerating Premixed Flame}

The morphology and propagation of premixed flames in obstructed (Bychkov) channel with both ends open show unique and distinct characteristics as compared to those in the semi-opened case. Initially, an oscillating trend was observed for $R e=12$, with this pulsating trend dependent on the blockage ratio, thermal expansion ratio and obstacle spacing. The series of snapshots in Fig. 7.1 shows the flame development and the flow distributions for a channel with $\operatorname{Re}=48, \alpha=1 / 2$, $\Theta=8$. The initial planar flame undergoes folding as the flame front propagates due to influence of thermal expansion across the front, while the entire flows are directed towards the left extreme as seen in Fig. 7.1a. Eventually, after propagating for about two channel width, see Fig. 1b, the flow is distributed between the two extremes and flame front gradually attained a tulip shape. The momentum and the heat losses from the left extreme moderate flame propagation significantly.
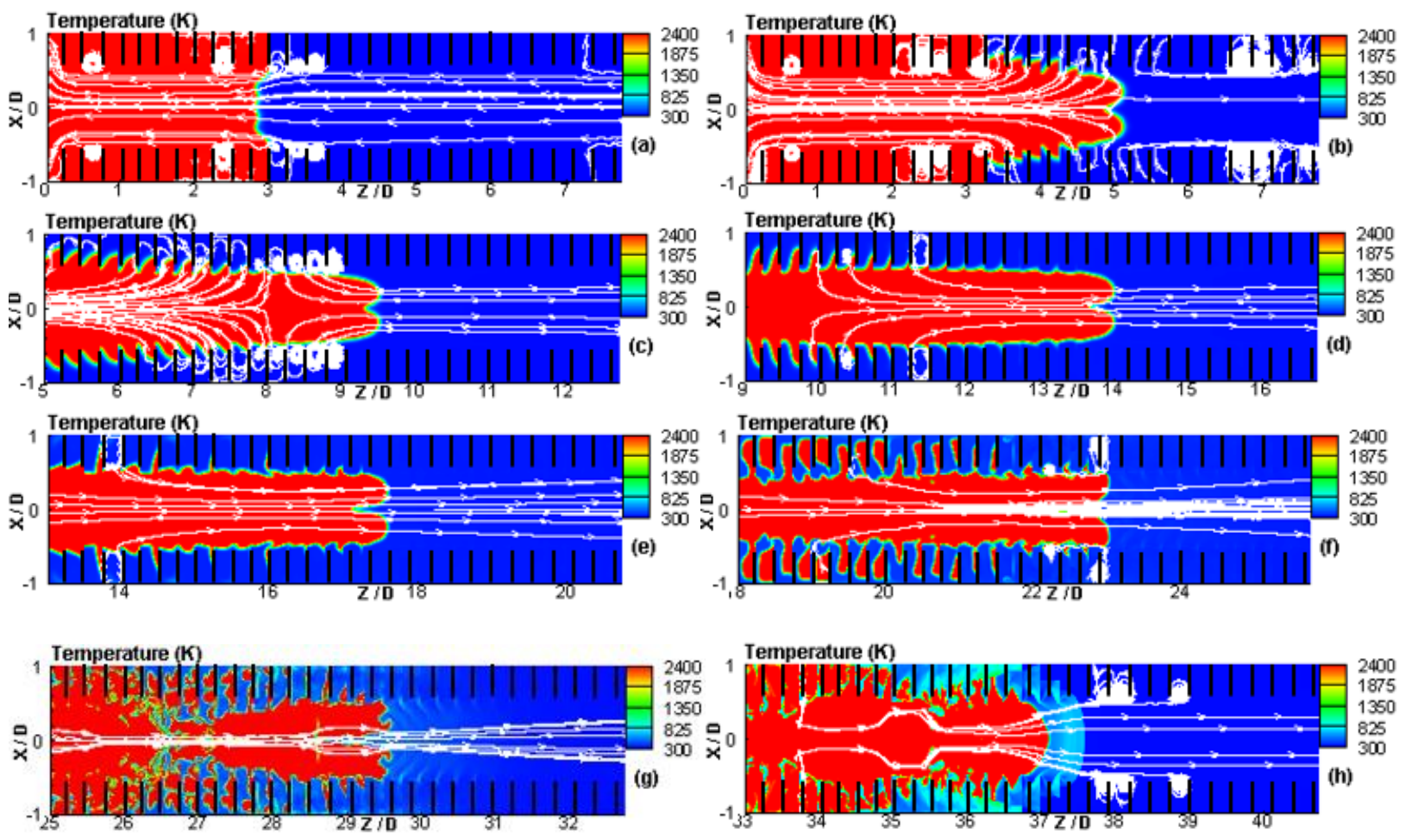

Figure 7.1: Flame development for $\alpha=1 / 2, \operatorname{Re}=48, \Theta=8$. 
The pockets of the unburnt fuel mixture are generated as the flame propagates (as seen in Fig. 7.1), which create delayed thermal expansion effects from each of these pockets. As the gas pockets start burning, the expanding gases flow into the core of the channel and are distributed with the main flow between the two extremes. With further propagation away from the left extreme, the unburned pockets are farther away, as shown in Figs. 7.1c-7.1d, the unburned pockets are further away from the left outlet, and the new gas volume from the thermal expansion effects flows towards the part of least resistance in the direction of the flame front. At this regime, the flame starts undergoing significant accelerations, this acceleration is sustained as the flame travels further, with new pocket formation isolated from the left extreme, thereby propelling it further ahead through a formation of a jet flow in the core path of the channel. This regime of burning is observed in Figs. 7.1e - 7.1g. As burning progresses further, the flame velocity grows, and the flame shape develops into a highly corrugated structure as seen in Figure 7.1g. With increasing Mach number, the influence of gas compressibility becomes significant, subsequently causing moderation of FA and saturation of the flame velocity to a near-constant value. Interestingly, at the saturated speed state, the flame front appears more stable with the disappearance of small-scale wrinkles as seen in Fig. 7.1h.

\subsection{Characteristics of the Accelerating Premixed Flame}

These observations provide insights into the physics of premixed flames in obstructed channels with both ends open. Namely, a flame undergoes initial quasi-steady propagation, which dominates in the region of the simultaneous (near equal) flow of the expanding gases in both extremes of the channels. Subsequently, the onset of FA occurs, which is then supplanted by the state of saturated velocity propagation. Figure 7.2a shows the propagation of the flame tip scaled by the channel half-width. To visualize these regimes clearly, it is important to investigate the derivative of the flame tip position (i.e. scaled flame tip velocity). As such, the scaled flame tip velocity is presented in Fig. 7.2b, indicating quasi-steady propagation, exponential acceleration and the saturated flame velocity propagation regimes. The flame tip variation with time shows qualitative similarity to the experimental results of Yanez et al. [34] in Fig. 7.3. The exponential acceleration regime mimics the trend in the semi-opened channel with closely packed obstacles, which indicates exponential acceleration, with the absence of initial quasi-steady propagation. However, such initial quasisteady propagation is quite similar to the oscillatory regime identified in narrower channels in Chapter 6. Apparently, the oscillations can correlate with prolonged quasi-steady propagation as 
the flame tries to balance the complex flow dynamics and gas expansion in such a highly confined space. Figures 7.4 and 7.5 compare the flame structure at the accelerating phase to that at the saturated phase. The leading vortices, inherent during the accelerating phases, are absent at the saturated phase, with strong vortices confined behind the flame front. Likewise, the leading shock waves in the accelerating phase are not apparent when the flame velocity saturates to a steady one.

(a)

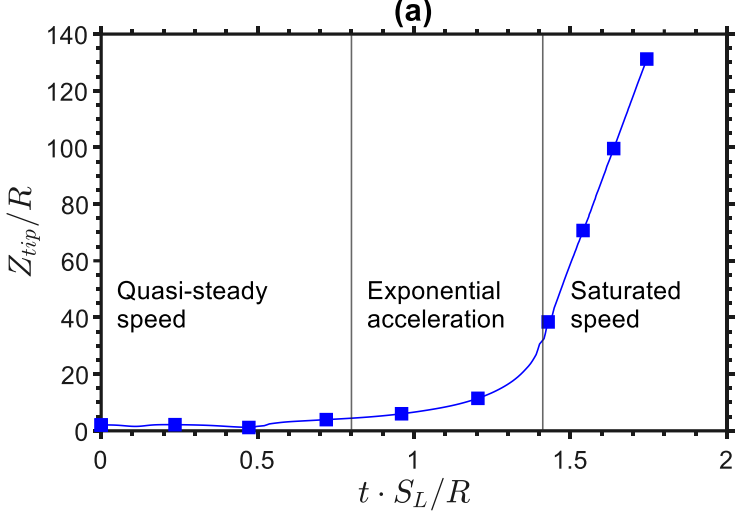

(b)

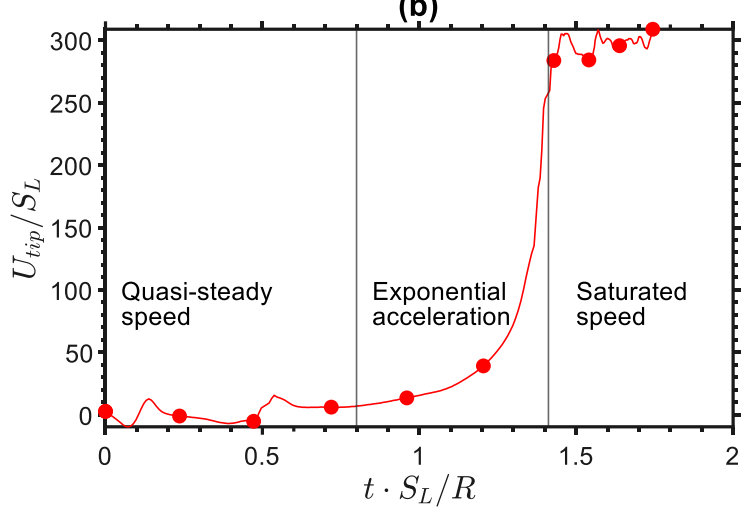

Figure 7.2: Various flame propagation trends for $\alpha=1 / 2, \Theta=8$ : a) $Z_{\text {tip }} / R$ b) $U_{\text {tip }} / S_{L}$.

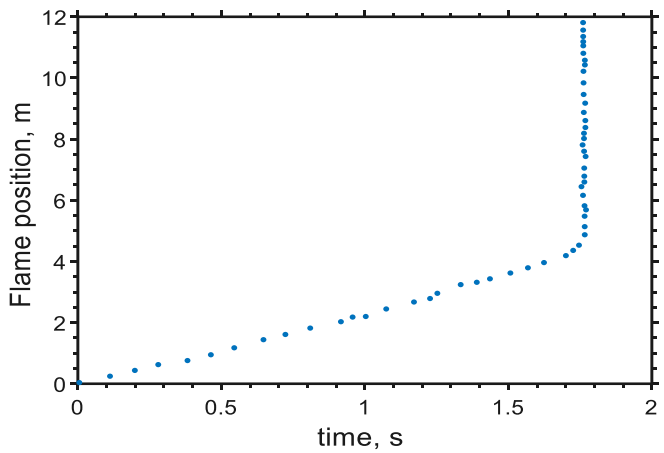

Figure 7.3: A flame propagation trend for $\alpha=0.6$ [34].
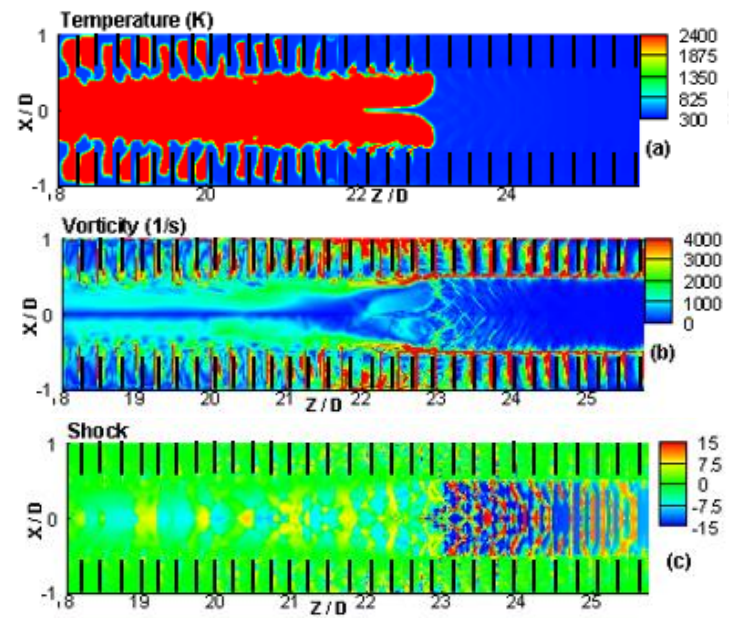

Figure 7.4: An accelerating flame for $\alpha=1 / 2, \Theta=8$. 

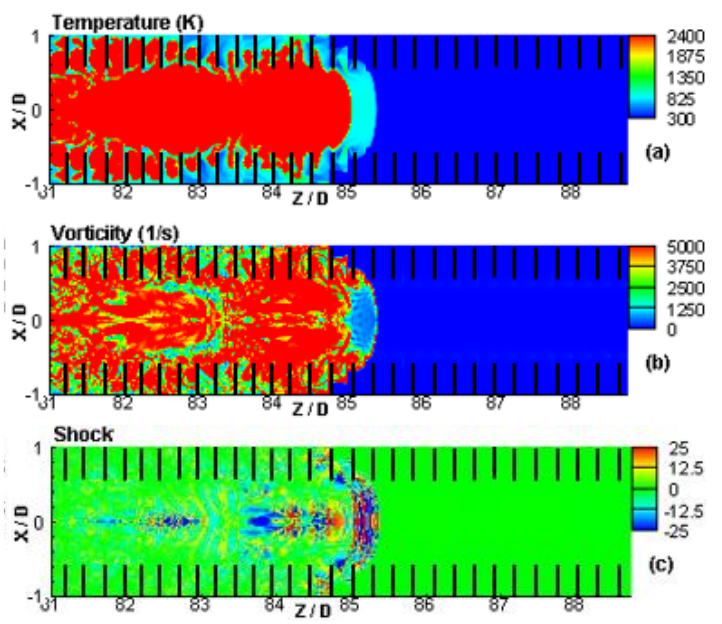

Figure 7.5: A saturated flame for $\alpha=1 / 2, \Theta=8$.

\subsection{Influence of the Geometrical and Thermochemical Factor}

The effects of blockage ratio and the channel half-width as well as the thermal expansion ratio on flame propagation are investigated. Figures $7.6-7.8$ show the scaled flame tip position and its scaled velocity versus time. The propagation trend is strongly influenced by the blockage ratios. Namely, as $\alpha$ increases, the duration of quasi-steady propagation also increases. For the lowest blockage ratio considered, $\alpha=1 / 3$, a flame propagates quasi-steady without acceleration. For low $\alpha$, the core (unobstructed) flow area is increased as such the volume of gas flowing out increases accordingly, and since the new volume of gases created in the pockets are reduced, the contribution of delayed burning to propelling the flame front decreases, causing reduction in the gas volume flowing towards the flame, thereby moderating the flame velocity tremendously.

(a)

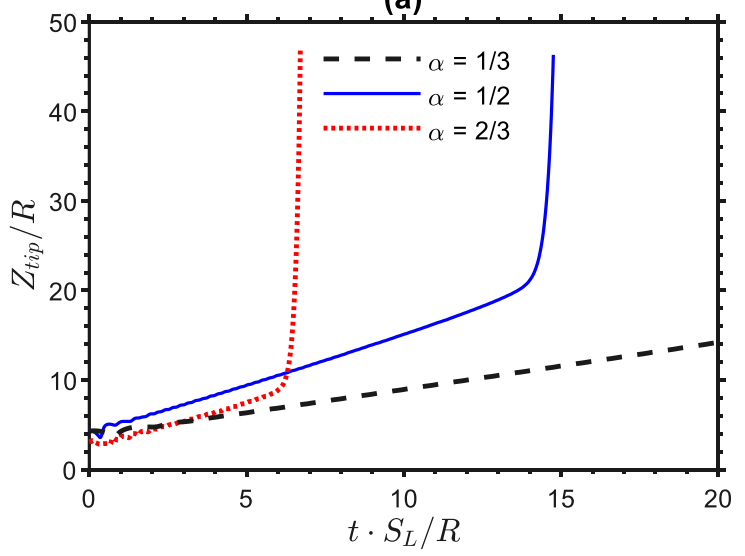

(b)

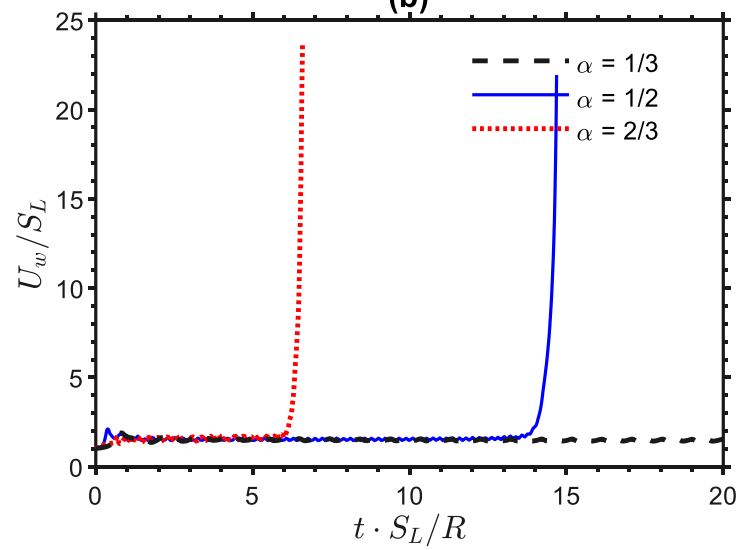

Figure 7.6: Flame propagation for $\Theta=8, R=24 L_{f}$ : a) $Z_{\text {tip }} / R$ vs $t \cdot S_{L} / R$ b) $U_{w} / S_{L}$ vs $t \cdot S_{L} / R$. 
(a)

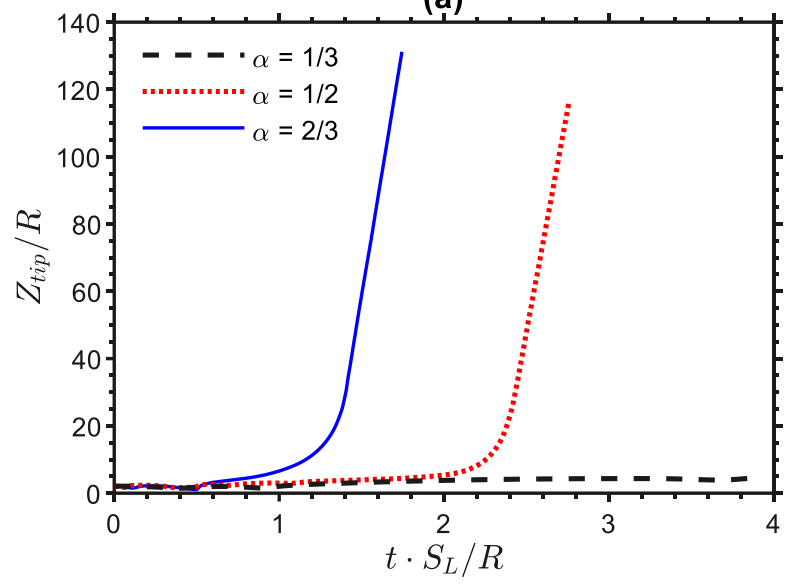

(b)

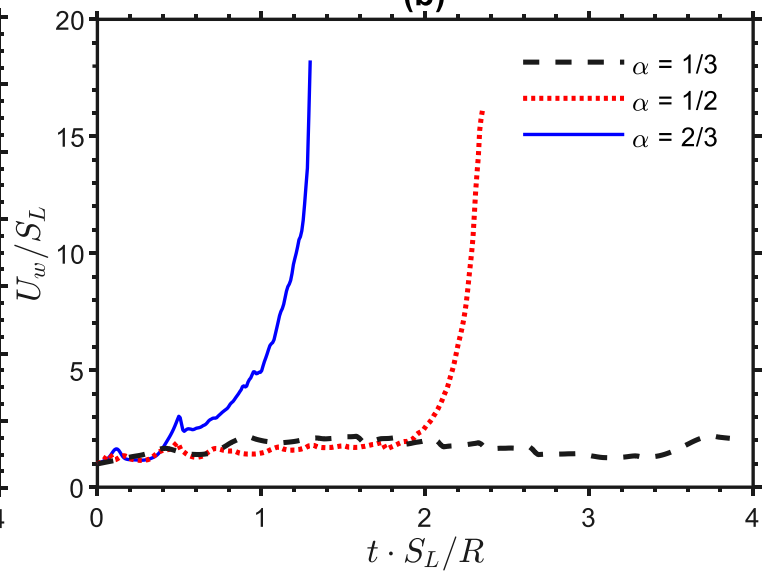

Figure 7.7: Flame propagation for $\Theta=8, R=36 L_{f}$ : a) $Z_{\text {tip }} / R$ vs $t \cdot S_{L} / R$ b) $U_{w} / S_{L}$ vs $t \cdot S_{L} / R$.

(a)

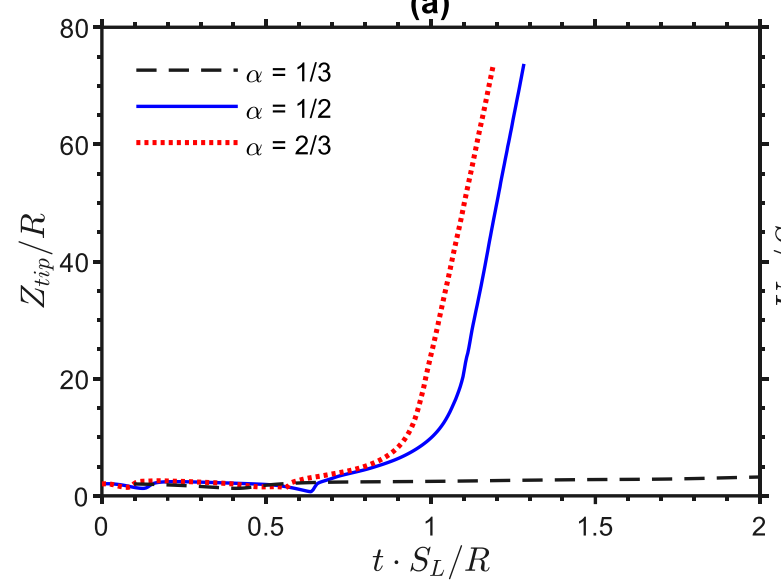

(b)

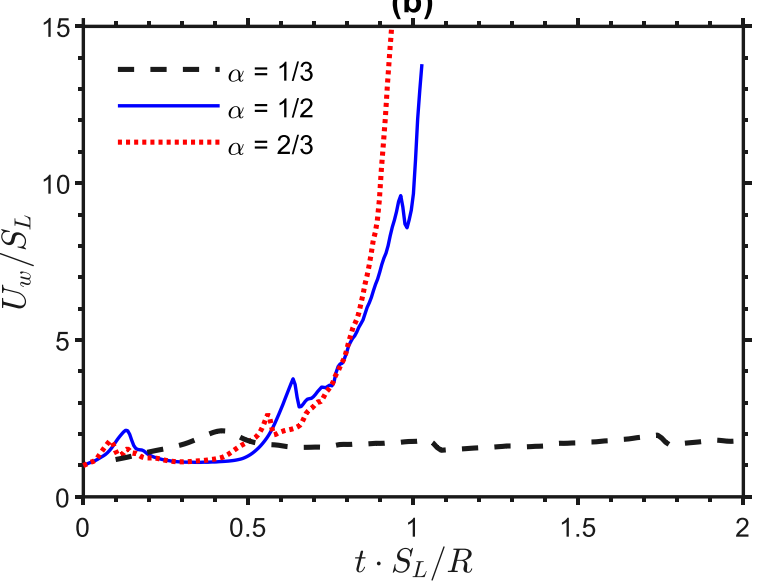

Figure 7.8: Flame propagation for $\Theta=8, R=48 L_{f}$ : a) $Z_{\text {tip }} / R$ vs $t \cdot S_{L} / R$ b) $U_{w} / S_{L}$ vs $t \cdot S_{L} / R$.

Moreover, increasing the channel half-width decreases duration of the regime of quasi-steady propagation for $\alpha=1 / 2,2 / 3$. This effect is observed more apparently in Fig. 7.9. Here, as the channel half-width increases from $24 L_{f}$ to $48 L_{f}$, the scaled time taken before acceleration starts decreases from 6 to 0.6. However, this time difference diminishes as the channel widens. Therefore, a growth of the channel width provides an impact similar to the effect of an increasing $\alpha$ on the shortening of the quasi-steady propagation regime. For a wider channel, of width $48 L_{f}$, both the blockage ratios $\alpha=1 / 2$ and $\alpha=2 / 3$ provide the similar effects on flame propagation and a difference in the durations of the quasi-steady propagation regimes is minor. 


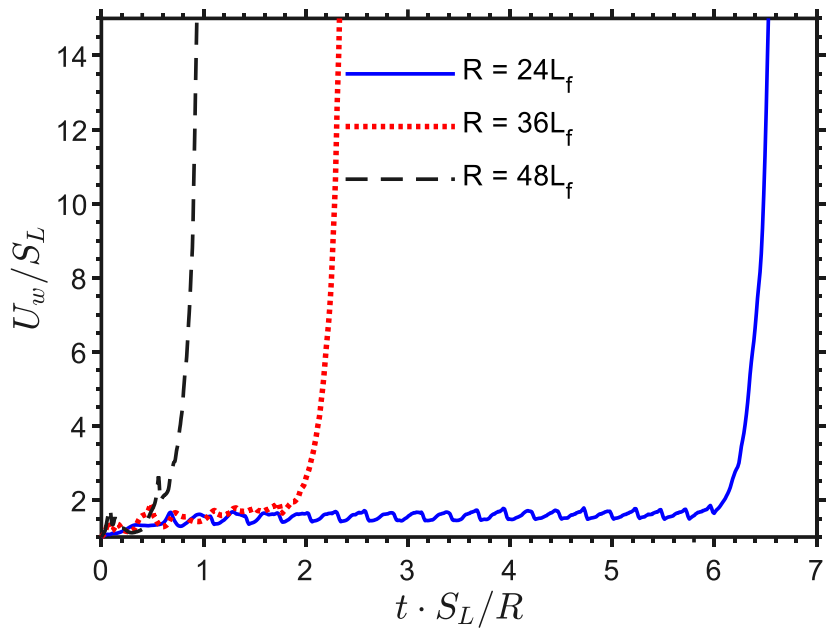

Figure 7.9: The scaled burning velocity $U_{w} / S_{L}$ versus the scaled time $t S_{L} / R$ for $\alpha=2 / 3, \Theta=8$.

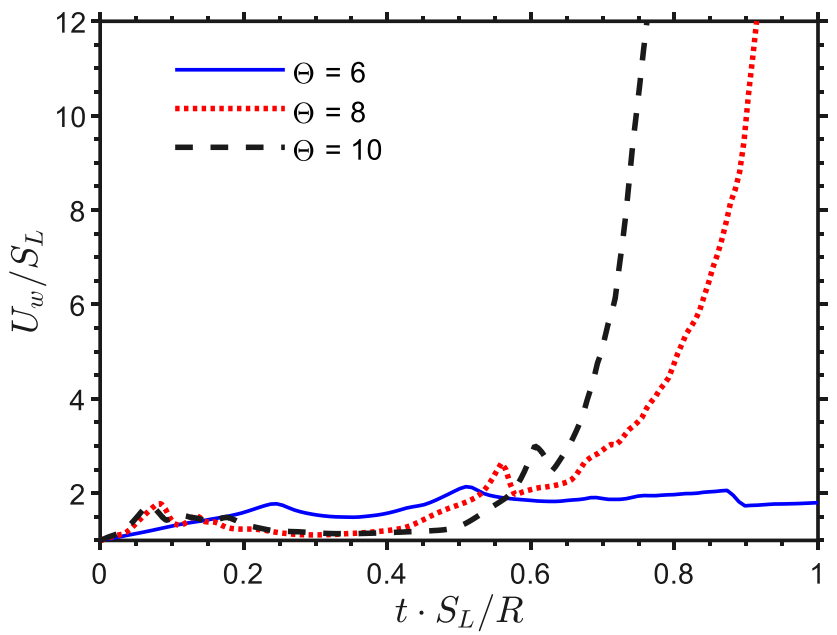

Figure 7.10: The scaled burning velocity $U_{w} / S_{L}$ versus the scaled time $t S_{L} / R$ for $\alpha=2 / 3, R=48 L_{f}$.

Similar to the effect of the blockage ratio, an increase in the thermal expansion ratio shortens the quasi-steady regime and the eventual acceleration rate is increased as shown in Fig. 7.10. For fuel mixtures of large thermal expansion ratio, the expansion produced across the flame is enhanced, and the flame area will grow accordingly leading to faster transition to the accelerating phase and faster accelerating flames. On the contrary, flames with lower thermal expansion ratio, propagates quasi-steadily without undergoing any significant accelerations.

\subsection{Validation of the Accelerating Flame Simulations by the Bychkov Theory}

Furthermore, exponential acceleration observed above correlates with the theory of Bychkov for flame in obstructed channel with both ends open presented in Eq. (2.9) - (2.10). The analytical solution for flame propagation in an obstructed channel with both ends open was developed 
considering inviscid and viscous flow. However, the viscous effect-based formulation, incorporates the channel length as one of the parameters, but in these simulations, the channel length is not defined as it grows with the flame, thereby limiting the validation of the viscositybased model. Since, the theory does not predict the quasi-steady propagation, the simulation is modified for consistency with the theory. As such, the flame tip position data are time-shifted, until the flame tip position $Z_{f}$ gives the best fit with the theory, Eq. (2.9). Consequently, time zero correlates to the beginning of exponential acceleration after eliminating the quasi-steady regime. These comparisons are shown in Figs. 7.11 - 7.13. Specifically, Fig. 7.11 present the trend in a channel of half-width $24 L_{f}$. While Fig. 7.11a shows a good prediction of the later acceleration phase when $\alpha=1 / 2$, it is seen in Fig. 7.11b, for $\alpha=2 / 3$, that the theoretical curve eventually deviates from the simulation results, at a later stage, but it shows better prediction at the early stage of the process.
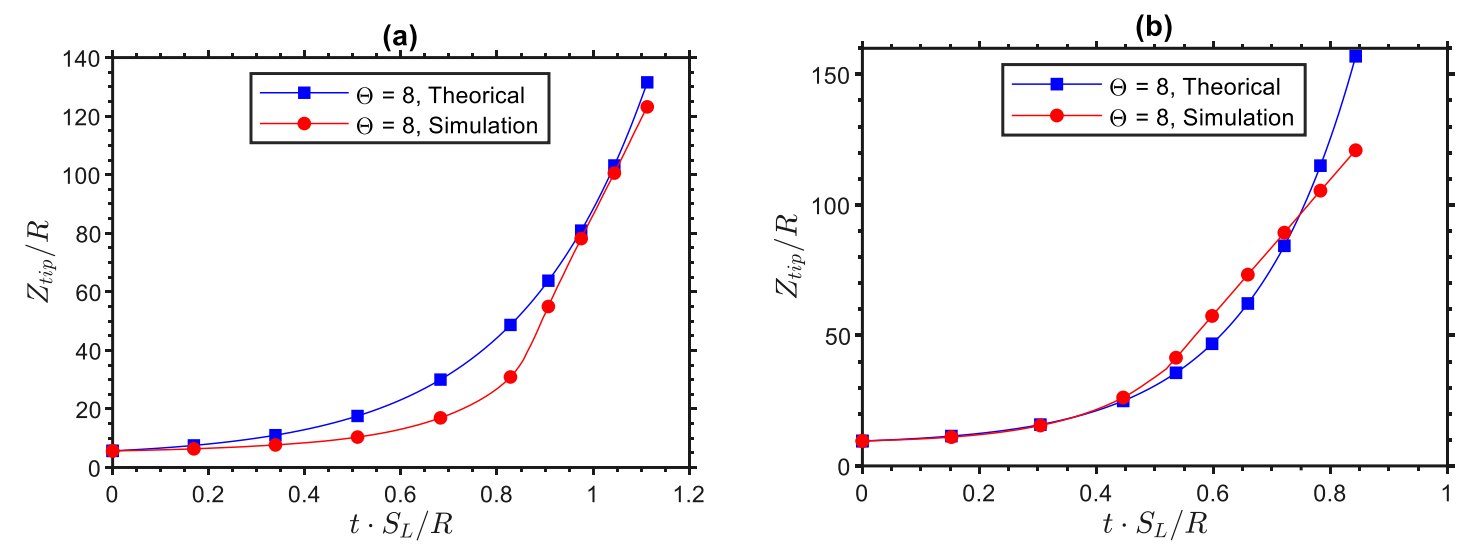

Figure 7.11: The scaled flame tip position versus the scaled time for $R=24 L_{f}:$ a) $\alpha=1 / 2$ b) $\alpha=2 / 3$.
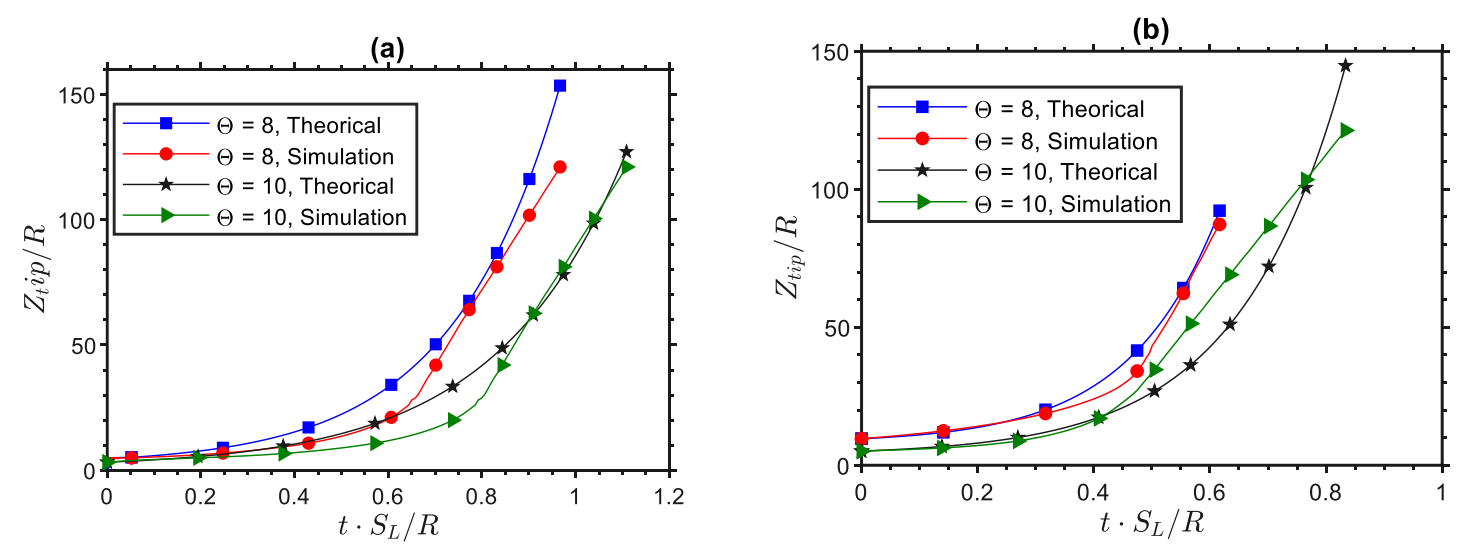

Figure 7.12: The scaled flame tip position versus the scaled time for $R=36 L_{f}:$ a) $\alpha=1 / 2$ b) $\alpha=2 / 3$. 

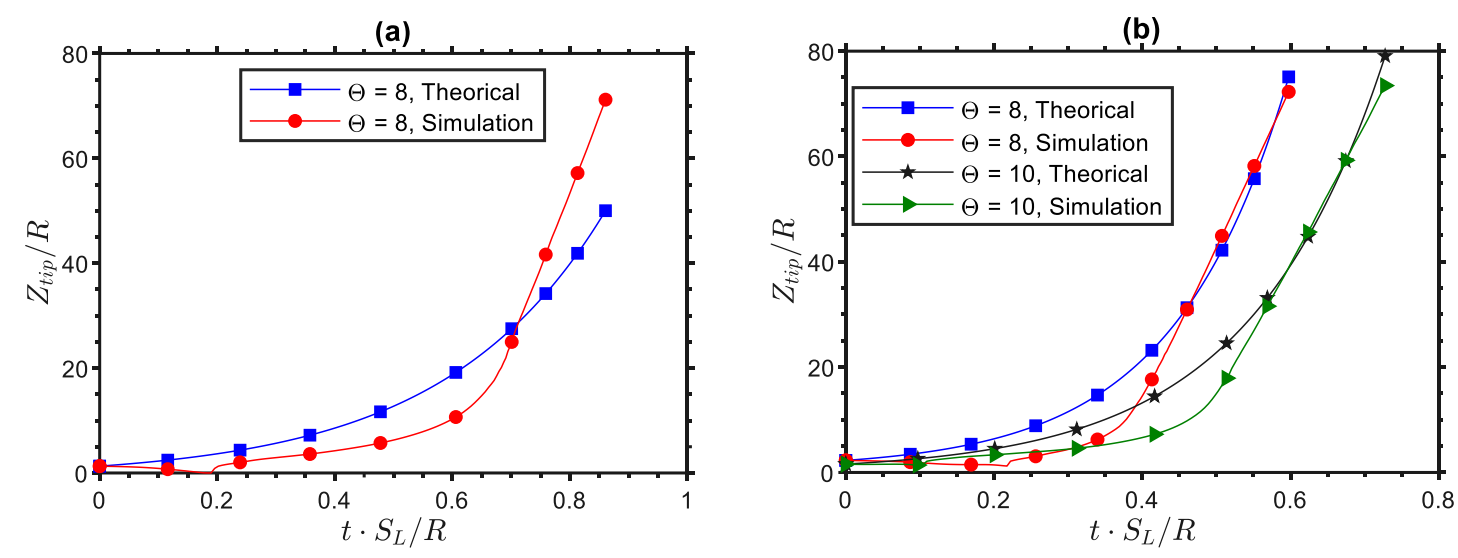

Figure 7.13: The scaled flame tip position versus the scaled time for $R=48 L_{f}:$ a) $\alpha=1 / 2$ b) $\alpha=2 / 3$.

In Figure 7.12a, representing the channel of half-width $36 L_{f}$ and $\alpha=1 / 2$, the theory captures the later trend better for $\Theta=10$. However, the later acceleration trend is better captured when $\Theta=8$, for the $\alpha=2 / 3$ case in Fig. 7.12b. The trends for $\alpha=1 / 2,2 / 3$ and $\Theta=8,10$ in the channel of half-width $48 L_{f}$ are shown in Fig. 7.13. The later trend of exponential acceleration is better captured for $\alpha=2 / 3$, Fig. 13b, and $\Theta=8,10$. Although the theory gives a good approximation of exponential acceleration, it narrowly captures the trend in the earlier phase of the acceleration. Theoretically, exponential acceleration rates have definite value even for low blockage ratio and thermal expansion ratio. For instance, for $\alpha=1 / 3, \Theta=6$, the exponential acceleration rate $\sigma_{0}=$ 2.17. However, no observable acceleration occurs with this set of parameters. The simulation results for this combination of such a low blockage and thermal expansion ratios do not show an accelerating flame. Moreover, acceleration is only noticeable when both $\alpha, \Theta$ are larger. At $\alpha=$ $1 / 2, \Theta=8$, acceleration becomes noticeable, when the $\sigma_{0}=3.66$. Therefore, the initiation of the actual exponential acceleration will happen when $2.17<\sigma_{0}<3.66$ which corresponds to $1 / 3<$ $\alpha<1 / 2$ and $6<\Theta<8$.

\subsection{Analysis of the Saturated Flame Regime}

The resulting saturated velocity occuring due to gas compression is expected to be lower than the Chapman-Jouget (CJ) deflagration velocity, which is defined by [16]

$$
\frac{U_{C J}}{c_{S}}=\left[1+\frac{\gamma(\gamma-1)}{2(\gamma+1)}\right] \sqrt{2\left(\frac{\Theta-1}{\gamma+1}\right)} .
$$

Figures $7.14 \mathrm{a}$ and $7.14 \mathrm{~b}$ present the scaled flame tip velocity versus the scaled flame tip position (such a phase diagram) for $\Theta=8$ and $\Theta=10$, respectively. These velocities are compared to the 
CJ deflagration velocity, $U_{C J}$, shown by the constant straight line on the figures. It is seen that the satured flame velocities are larger for $\alpha=1 / 2$ as compared to those $\alpha=2 / 3$ and independent of the tube radius particularly for $\Theta=10$. In the case of $\Theta=8$, the observed saturated velocity is slightly higher for $24 L_{f}$ than $36 L_{f}$ case as seen in Fig. 7.14a. However, the saturated velocity collapses to the same value for all width for each blockage ratio $\alpha=1 / 2$ and $\alpha=2 / 3$ when $\Theta=$ 10, as shown in Fig. 7.14b. Finally, all the accelerating cases shows that the saturated speed are much less than the CJ deflagration velocity.
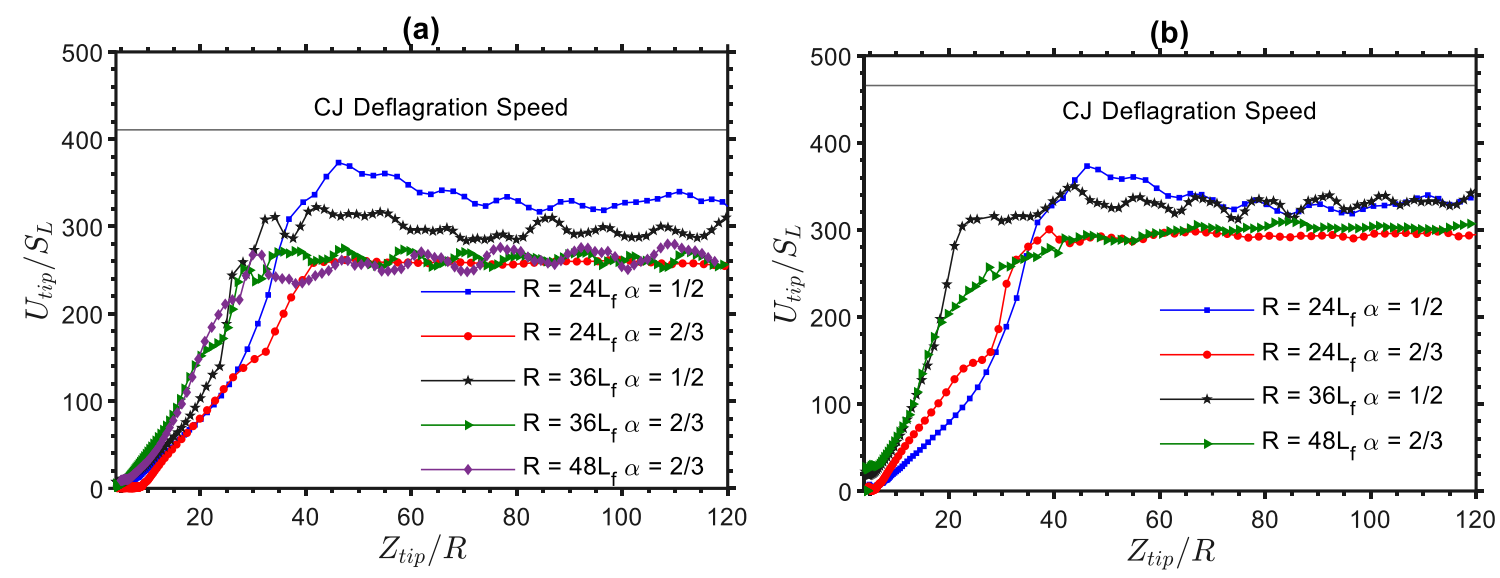

Figure 7.14: The scaled flame tip velocity versus its scaled position: a) $\Theta=8$ b) $\Theta=10$.

\subsection{Classification of the Flame Propagation Regimes}

To develop a predicted model for the propagation trends observed in obstructed channels with both ends open, a machine learning "logistic regression algorithm" is adopted to train these data sets in order to classify accelerating versus non-accelerating flame trends considering $\Theta, \alpha$ and $R$ as the parameters. Moreover, the model will indicate the point when a flame accelerate or oscillate based on the channel width or the flame Reynolds number.

Figure 7.15 is a schematic of the machine learning model. The procedure involves feeding the learning algorithm with the training sets (thermal expansion ratio, blockage ratio and/or the flame Reynolds number and the classification variable). Here, the classification variable indicates accelerating or not accelerating event. So, the algorithm uses this data set to learn a hypothesis function, $h$, that maps input $x$ to the predicted output $y$. 


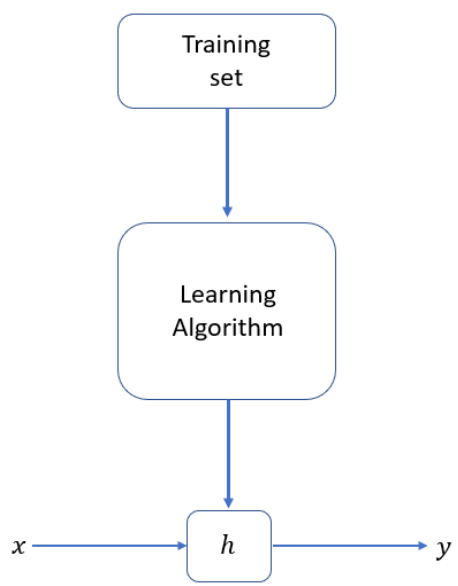

Figure 7.15: Machine learning model representation.

Let us consider a hypothesis function in logistic regression.

$$
h_{\theta}(x)=g\left(\theta^{T} x\right), \quad 0 \leq h_{\theta}(x) \leq 1,
$$

where the sigmoid function $g$ is defined as

$$
g(z)=\frac{1}{1-e^{-z}}, \quad z=\theta^{T} x .
$$

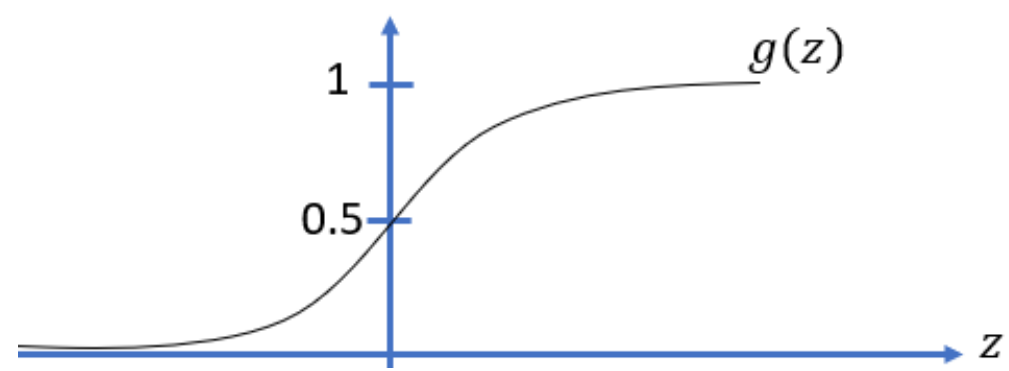

Figure 7.16: Sigmoid function

As a result, the function $h_{\theta}(x)$ decribes the probability that $y=1$ for a given $x$ value. As such, when $h_{\theta}(x) \geq 0.5$, then $y=1$, while $y=0$ for $h_{\theta}(x)<0.5$. From the simulation results, the cases undergoing acceleration are classified as 1 , and the cases with only quasi-steady propagation are 0 . The training sets consisting of the input variable $x(\Theta, \alpha, R e)$ and $y$.

The result obtained for the hypothesis function are then used to classify and develop the regime diagrams as depicted in Figs. 7.17 - 7.21. Specifically, Fig. 7.17 shows the classification results obtained for $R e=24$. The line indicates the decision boundary represented by the variable $z$. Essentially, the equation satisfying the accelerating phase is $-131.69+9.36 \Theta+112.29 \alpha \geq$ 0 with a training accuracy of $91.67 \%$. For $R e=36,48$ in Figs. 7.18, 7.19 the predicted hypothesis 
is the same, and the decision boundary is represented by $-111.73+10.19 \Theta+69.85 \alpha \geq 0$ with the training accuracy $100 \%$.

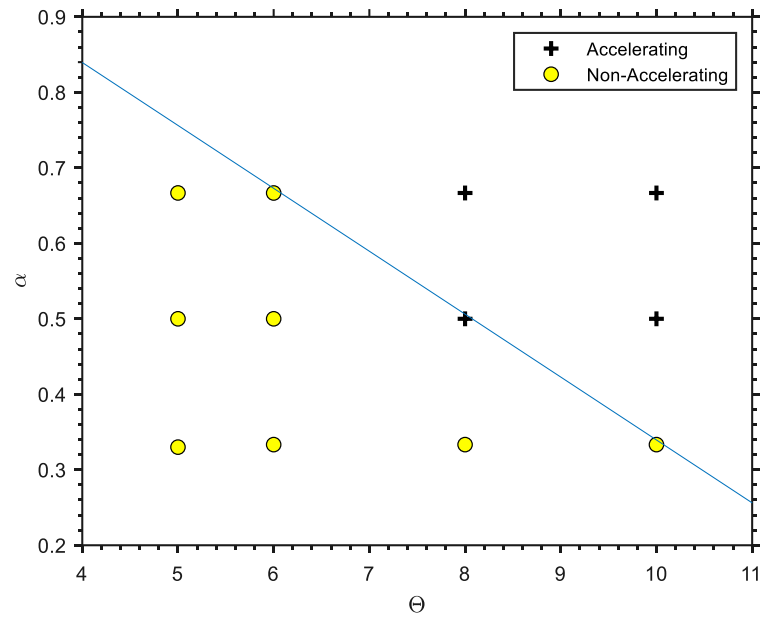

Figure 7.17: Classification of flame propagation for $R e=24$.

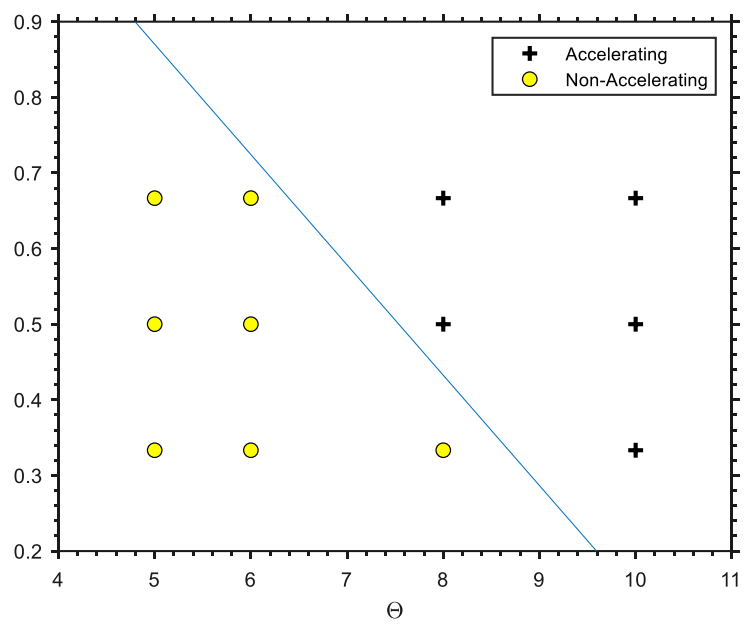

Figure 7.18: Classification of flame propagation for $R e=36$.

Finally, the data are classified based on $R e$ and the model developed predicts if a flame accelerates or not based on the flame Reynolds number Re. Figures 7.20 and 7.21 show this result for $\Theta=8$ and $\Theta=10$, respectively. The model prediction is therefore $-17.01+0.19 \Theta+22.62 \alpha \geq 0$, with $83.3 \%$ prediction accuracy for $\Theta=8$ and $-14.4+1.14 \Theta+39.9 \alpha \geq 0$, with a $100 \%$ prediction accuracy for $\Theta=10$. 


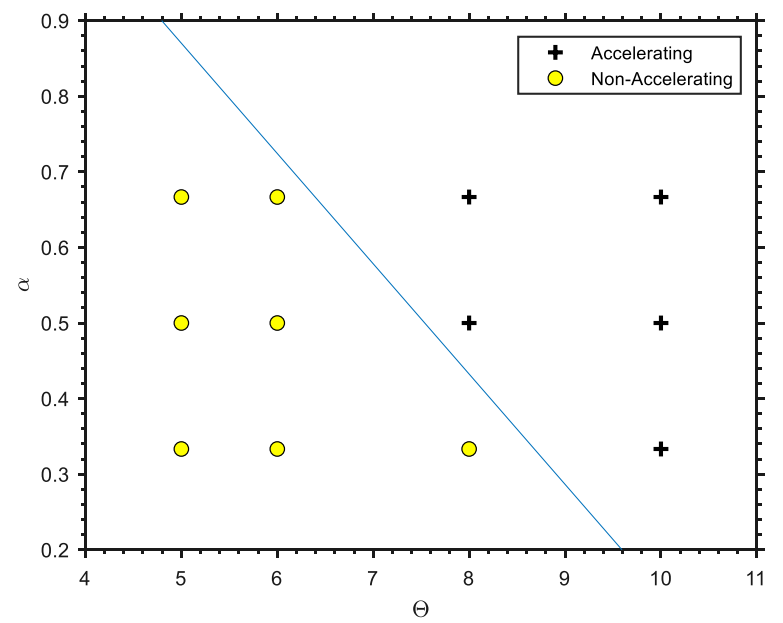

Figure 7.19: Classification of flame propagation for $R e=48$.

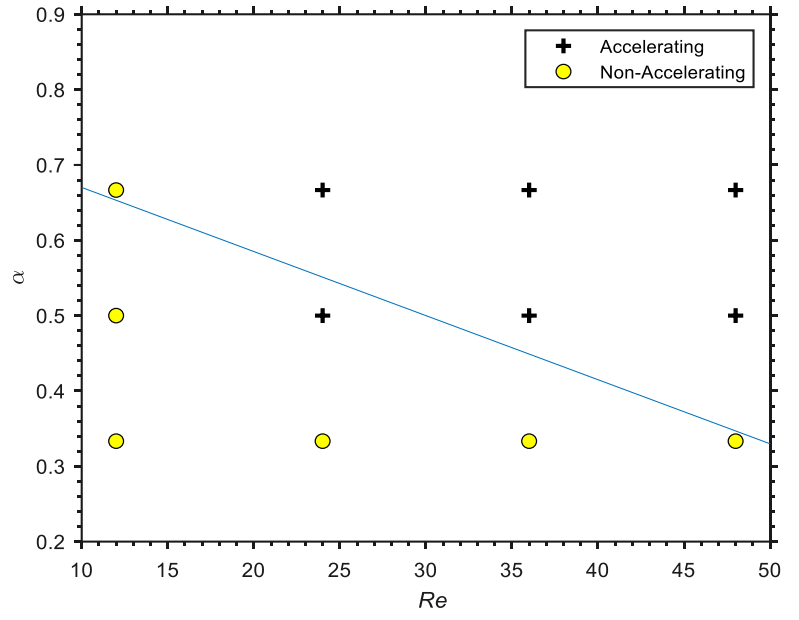

Figure 7.20: Classification of flame propagation for $\Theta=8$.

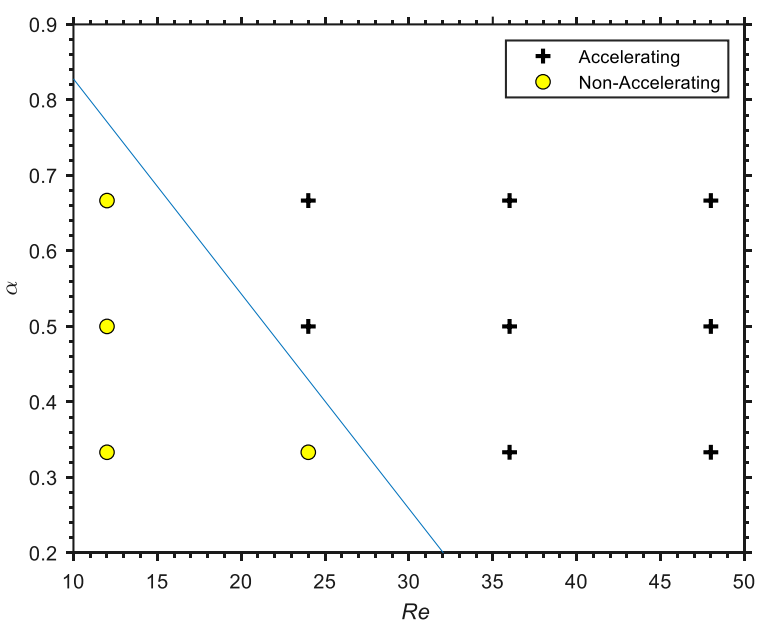

Figure 7.21: Classification of flame propagation for $\Theta=10$. 


\section{Modeling of Supercritical $\mathrm{CO}_{2}$-Diluted Oxy-Methane Flames}

\subsection{Details of the Parametric Study}

The present computational simulations were conducted with the modified version of the in-house research code based on the real gas model (3.13) - (3.25). The energy release in the chemical reaction $Q$ is given by Eq. (3.17), where the adiabatic flame temperature $T_{a d}$ is obtained from the chemical equilibrium model in Chemkin Pro, a commercial software for solving chemical kinetics problems [58]. The respective values of $T_{a d}$ are listed in Table 1 for various $\mathrm{CO}_{2}$-diluted mixtures. The initial pressure is $P_{f}=300 \mathrm{bar}$, with the initial temperature $T_{f}=800 \mathrm{~K}$. In the absence of reliable chemical kinetics data for methane $\left(\mathrm{CH}_{4}\right)$ combustion in the presence of diluted $\mathrm{CO}_{2}$ at these conditions, a one-step chemical kinetics model based on the Arrhenius reaction with the activation energy $E_{a}=235.8 \mathrm{~kJ} / \mathrm{mol}$ and the reaction time constant $\tau_{R}=1.72 \times 10^{-13} \mathrm{~s}$, which corresponds to stoichiometric methane-oxygen burning at 1 bar is utilized. The global chemical reaction reads

$$
\mathrm{CH}_{4}+2 \mathrm{O}_{2}+n \mathrm{CO}_{2} \rightarrow \mathrm{CO}_{2}+2 \mathrm{H}_{2} \mathrm{O}+n \mathrm{CO}_{2}, \quad \beta \equiv n /\left(n+n_{O 2}\right)=n /(n+2),
$$

where the rate of $\mathrm{CO}_{2}$-dilution $\beta$ ranging from $\beta=0$ for pure methane-oxygen combustion till $\beta=80 \%$. We consider a 2D semi-open channel, with one end closed and the other being open, with non-reflective boundary conditions considered, and with a planar flame propagating from the closed towards the open end. The walls are either slip, $\bar{n} \bullet u=0$, to keep the flame planar, or nonslip, $u=0$, which corrugates the flame front. The channel widths are $D=5,10,20,40 \mu \mathrm{m}$.

\subsection{Planar Flames}
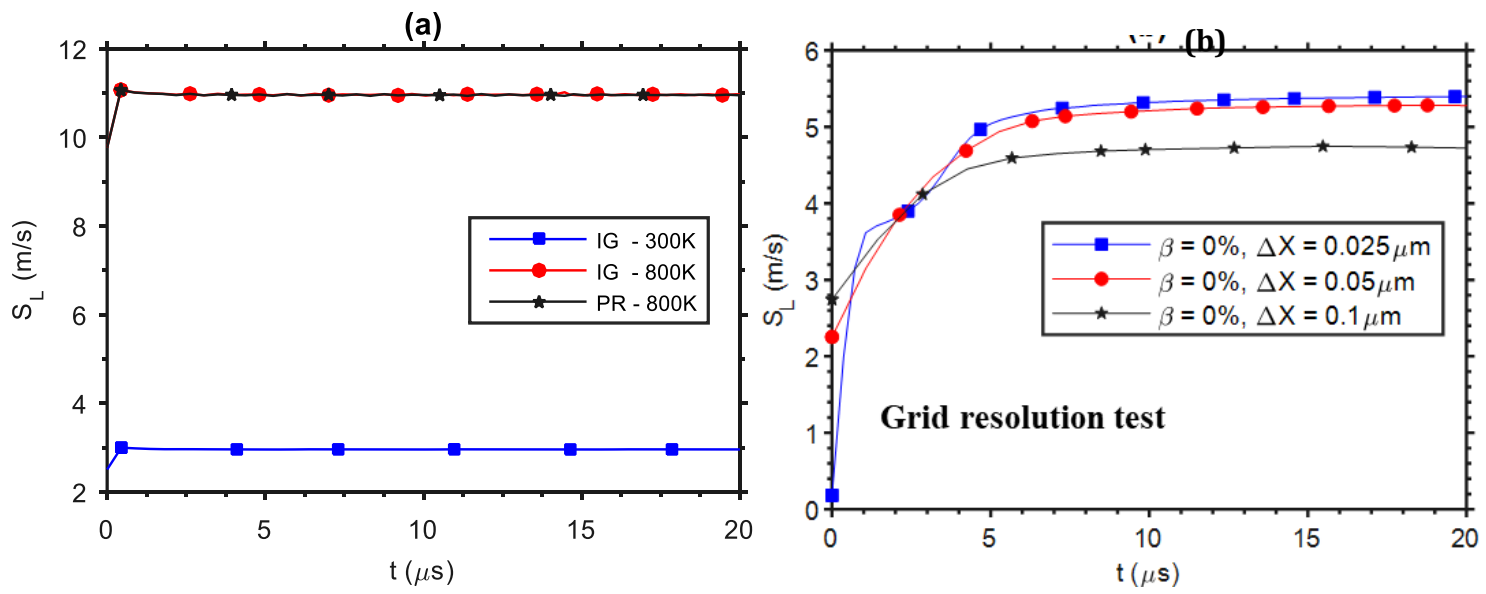

Figure 8.1: Planar flame speeds vs time at $P_{f}=1 \mathrm{bar}$ a) comparing of IG with PR b) grid resolution check. 
Table 8.1: Chemical heat release values:

\begin{tabular}{|c|c|c|c|c|c|}
\hline$\beta(\%)$ & 0 & $20 \%$ & $40 \%$ & $60 \%$ & $80 \%$ \\
\hline$n(m o l)$ & 0 & 0.5 & 1.33 & 3.0 & 8.0 \\
\hline$T_{b}(K)$ & 3956 & 3684 & 3343 & 2889 & 2079 \\
\hline$Q(\mathrm{MJ} / \mathrm{kg})$ & 6.56 & 5.64 & 4.39 & 3.34 & 1.84 \\
\hline
\end{tabular}

(a)

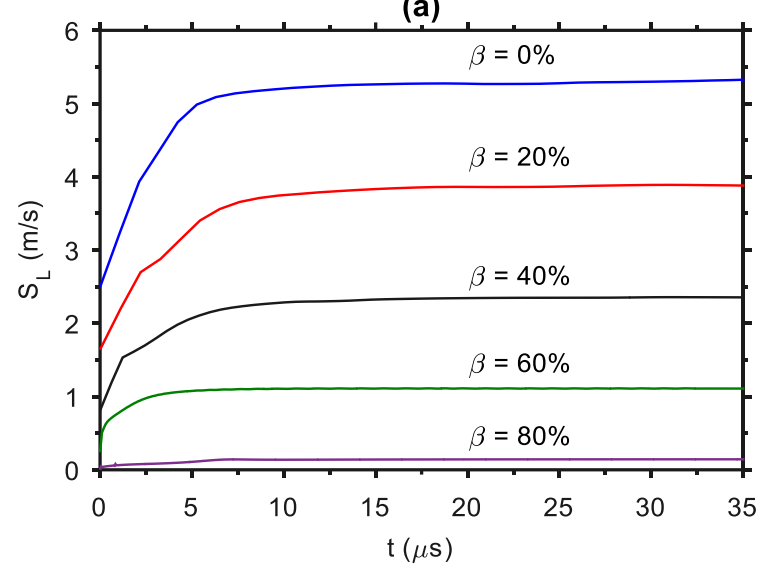

(b)

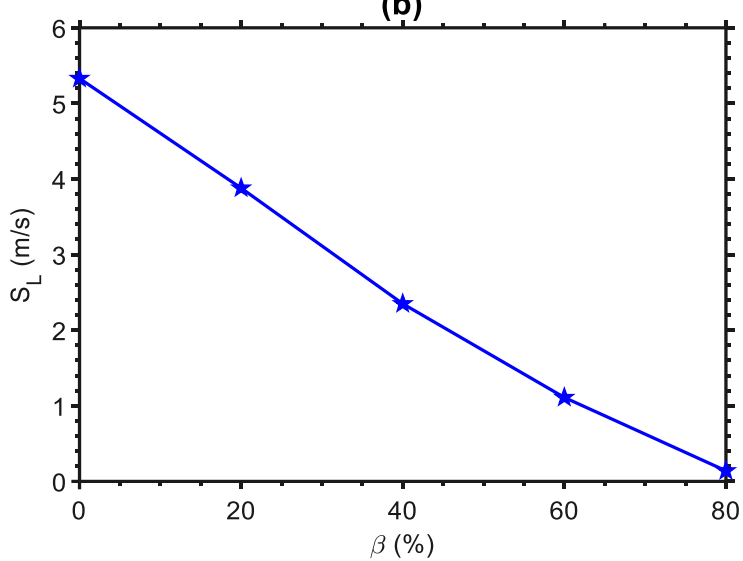

Figure 8.2: Planar flame speeds for $T_{f}=800 K, P_{f}=300 \mathrm{bar}$ (a) speed vs time (b) speed vs dilution rates

The free-slip boundary conditions were implemented to prevent flame corrugation due to wall friction, to obtain the planar flame speeds (speed relative to the unburnt fuel mixture) $S_{L}$. To validate the computational platform, the conventional condition (the room temperature) $T_{f}=$ $300 \mathrm{~K}$, pressure $P_{f}=1$ bar with the ideal-gas (IG) EoS are considered at first, to reproduce the experimental measurements of the unstretched laminar flame speed for the stoichiometric oxymethane mixture [59], with $S_{L}=2.9 \mathrm{~m} / \mathrm{s}$. Figure 8.1a shows that the ideal gas simulation for $1 \mathrm{bar}$ and $300 \mathrm{~K}$ agrees very well with the experimental data. Also, both PR and IG EoS models predict the planar flame speeds at elevated temperature $T_{f}=800 \mathrm{~K}$, pressure $P_{f}=1$ bar computed for $\beta=0$ which are shown in Figure 8.1a. Before proceeding with the supercritical flame simulations $\left(T_{f}=800 \mathrm{~K}\right.$, pressure $\left.P_{f}=300 \mathrm{bar}\right)$, series of simulation runs are conducted to determine the grid size that best resolve the details of the flame. Therefore, grid cells of $\Delta X=$ $0.025,0.05,0.1 \mu \mathrm{m}$ were tested for $\beta=0$. The planar speeds decrease by $1.77 \%$ and $12.46 \%$ relative to when $\Delta X=0.025 \mu \mathrm{m}$ as shown in Figure 8.1b. In Fig. 8.2a, $S_{L}$ is presented as a function of $\beta$. The observed flame speed has a peak value of $S_{L}=5.4 \mathrm{~m} / \mathrm{s}$ when $\beta=0$ and reduces by 
about $97.4 \%$ to $S_{L}=0.14 \mathrm{~m} / \mathrm{s}$ when $\beta=80 \%$. Therefore, a linear trend exists between the planar flame speeds and the $\mathrm{CO}_{2}$ dilution rates as seen in Figure 8.2b.

Subsequently, the internal structure of the flame is explored. Figure 8.3 shows the variation of the temperature and density across the flame fronts for all cases of the dilution rates. A very high temperature and density gradients are observed, which decrease as the dilution rate increases. When $\beta>60 \%$, the gradients of both temperature and density are minimized. This observation favors the flame propagation rates significantly. Since, higher temperature and density gradients implies higher rates of diffusion which favors both heat and mass transfer from the hot zone to cold fuel and conversely. Moreover, the density profile in Figure 8.3 indicates that density of the gaseous fuel mixture is greater than when at atmospheric condition by $\sim 2$ orders of magnitude. The density approaches those of liquids at this highly supercritical condition and increases with $\beta$. Furthermore, at low dilution rates the thermal expansion ratio $\Theta$ (ratio between fuel mixture $\rho_{f}$ and burnt product $\rho_{b}$ density) are highest with $\Theta=4.52$ when $\beta=0$ and $\Theta=2.6$ for $\beta=80 \%$, as flame propagation rates decreases as the thermal expansion decreases. Likewise, the burnt matter temperature decreases with $\beta$. As observed in Figure $8.3 \mathrm{~b}$, the temperature diminishes from $3860 \mathrm{~K}$ at $\beta=0$ to $2047 \mathrm{~K}$ at $\beta=80 \%$. To fully characterize the planar flame, it is essential to compute the thickness of the flame for each of these cases. The flame thickness $L_{f}$ is computed from the classical formulation [60].

$$
L_{f}=\frac{T_{b}-T_{u}}{(\nabla T)_{\max }},
$$

where $T_{b}$ is the burnt matter temperature and $T_{u}$ is the temperature of unburnt fuel mixture. The dilution of the flame cools down the flame temperature, since the high-density $\mathrm{CO}_{2}$ fluids have larger specific heat capacities and the temperature of the burnt product drops with higher dilutions. Figure 8.4a also shows similar trends as Figure $8.3 \mathrm{a}-\mathrm{b}$. The large fuel mixture species mass fraction gradients in low diluted cases favors mass transport and the reaction rates, thereby leading to an increase in the flame speeds. In Figure $8.4 \mathrm{~b}$, the value $L_{f}$ increases with $\beta$, it grows from $0.21 \mu \mathrm{m}$ for $\beta=0$ till $3.53 \mu \mathrm{m}$ for $\beta=80 \%$. The flame speed decreases, causing an apparent thickening of the flame at a high $\mathrm{CO}_{2}$-dilution. The summary of results of the planar flame propagation are presented in Table 8.2. 
(a)

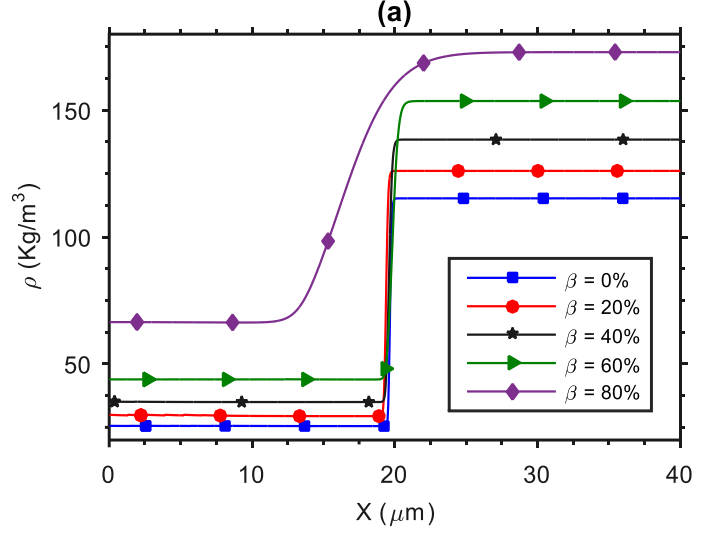

(b)

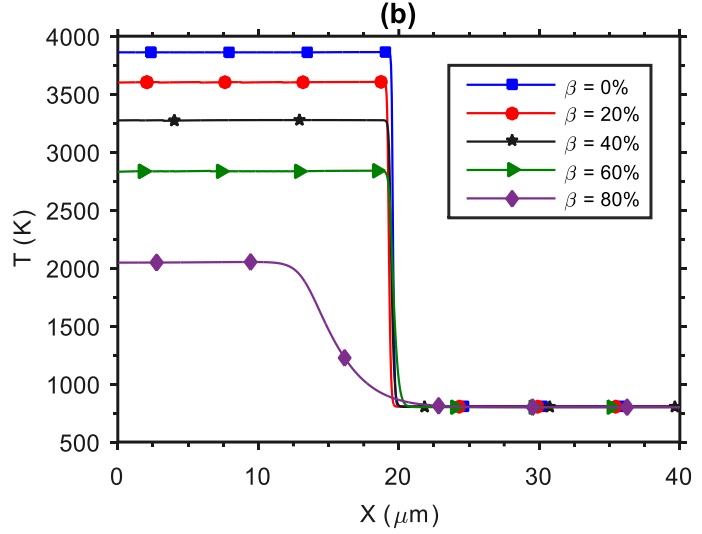

Figure 8.3: The density (a) and temperature (b) profiles at $T_{f}=800 \mathrm{~K}, P_{f}=300 \mathrm{bar}$.
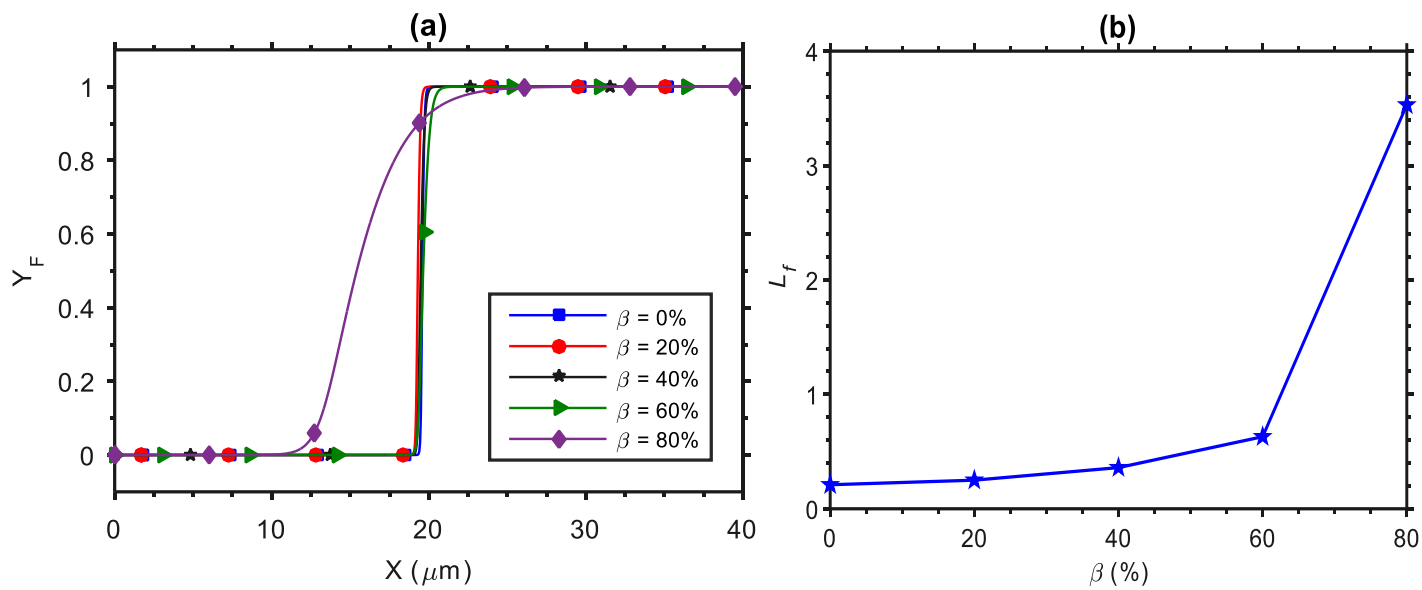

Figure 8.4: Profiles at $T_{f}=800 K, P_{f}=300$ bar (a) fuel mass fraction (b) flame thickness $\beta$

Table 8.2: Planar flame results

\begin{tabular}{|c|c|c|c|c|c|}
\hline$\beta(\%)$ & 0 & $20 \%$ & $40 \%$ & $60 \%$ & $80 \%$ \\
\hline$n(\mathrm{~mol})$ & 0 & 0.5 & 1.33 & 3.0 & 8.0 \\
\hline$S_{L}(\mathrm{~m} / \mathrm{s})$ & 5.4 & 3.88 & 2.35 & 1.11 & 0.14 \\
\hline$c_{f}(\mathrm{~m} / \mathrm{s})$ & 616.55 & 593.49 & 566.68 & 539.03 & 505.85 \\
\hline$\delta_{L}(\mu \mathrm{m})$ & 0.21 & 0.25 & 0.36 & 0.63 & 3.53 \\
\hline$\rho_{f}\left(\mathrm{~kg} / \mathrm{m}^{3}\right)$ & 115.4 & 126.17 & 138.4 & 153.63 & 172.91 \\
\hline$\eta_{f}(\mu \mathrm{Pa} \cdot s)$ & 37.59 & 37.82 & 38.19 & 38.70 & 39.42 \\
\hline$\Theta$ & 4.52 & 4.29 & 3.96 & 3.50 & 2.60 \\
\hline
\end{tabular}

\subsection{Corrugated Flames}

Next, the investigation is extended to cases of channels with nonslip wall conditions. As observed in Figure 8.5, the flame shape for the pure oxy-methane combustion and weak to mild $\mathrm{CO}_{2}$ diluted cases are concaved towards the fuel mixture such that they exhibit a tulip flame structure where the flame tip (the center point of the flame) points towards the burned matter and 
as the dilution rates increase the "strength" of the tulip flame or the flame concavity diminishes, and the flame gradually acquires a convex form.
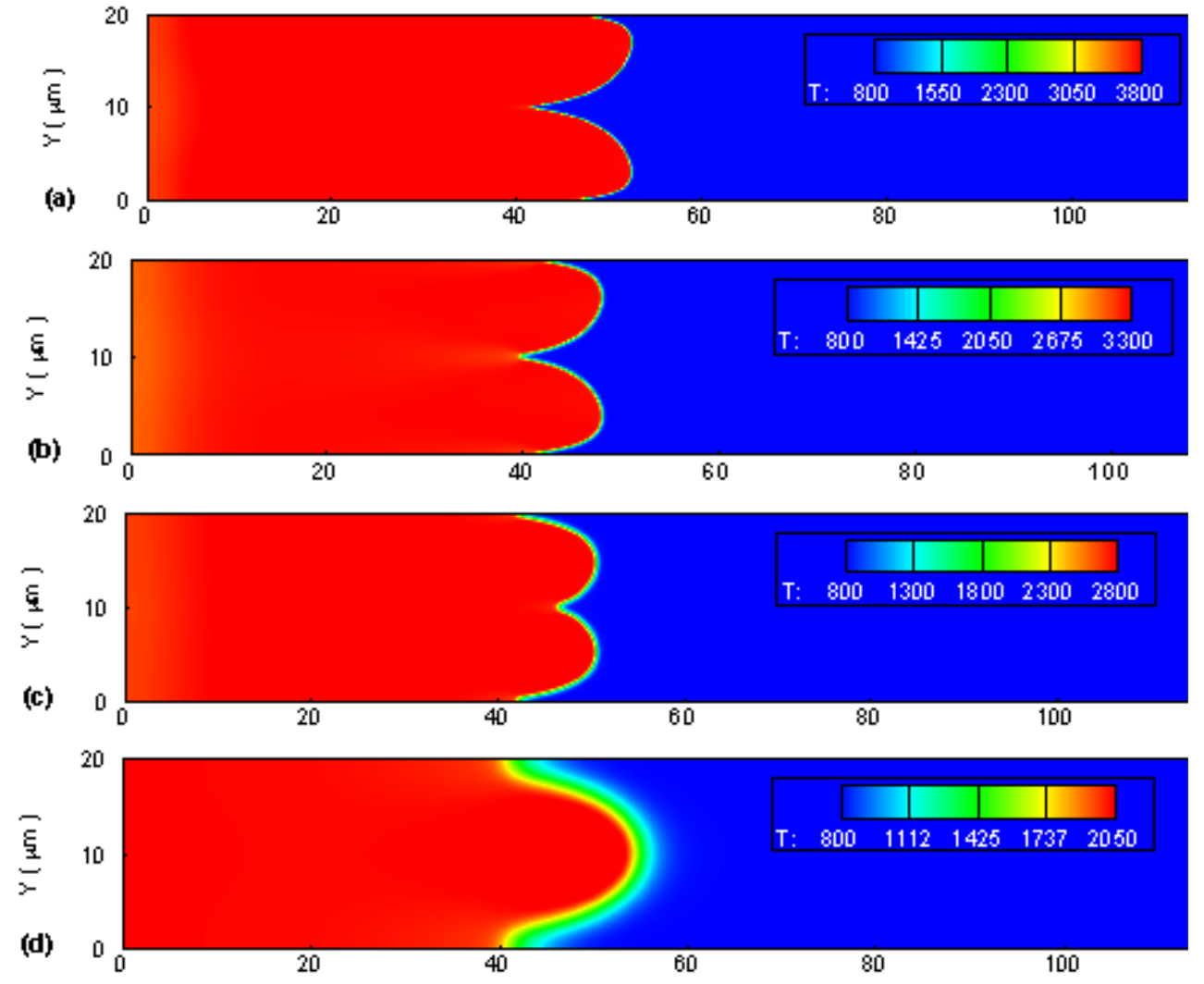

Figure 8.5: Flame snapshots in a $20 \mu \mathrm{m}$ channel (a) $\beta=0$ (b) $\beta=40 \%$, (c) $\beta=60 \%$, (d) $\beta=80 \%$
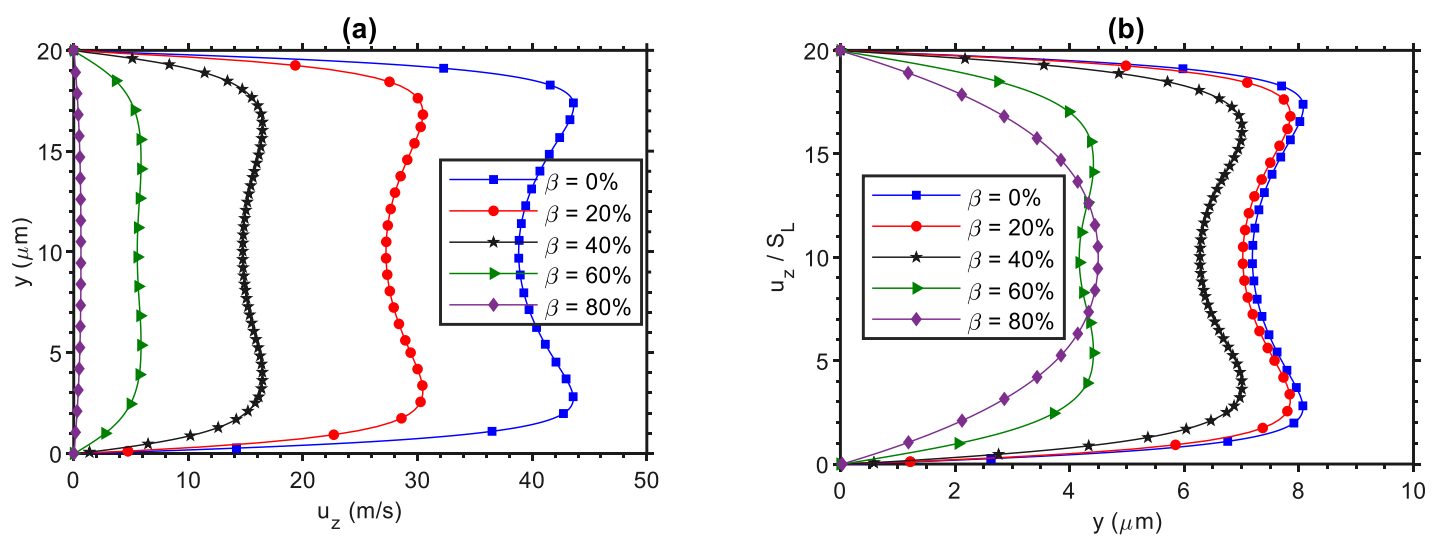

Figure 8.6: Velocity profile just ahead of the flame in a $20 \mu \mathrm{m}$ channel (a) $x$-component (b) scaled $x$-component. 
(a)

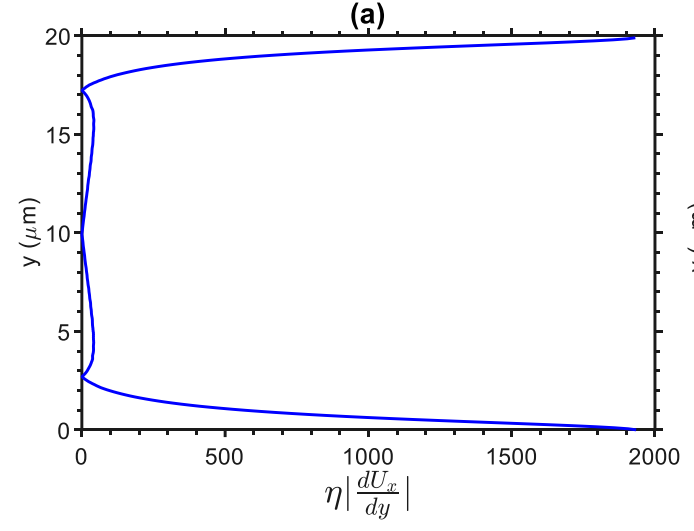

(c)

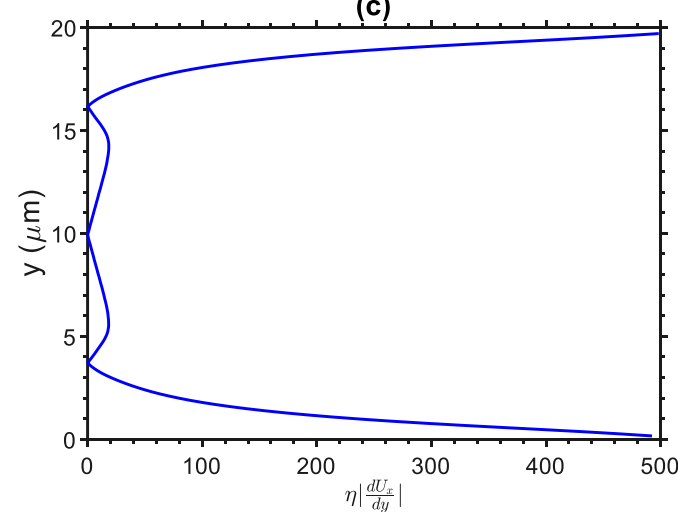

(e)

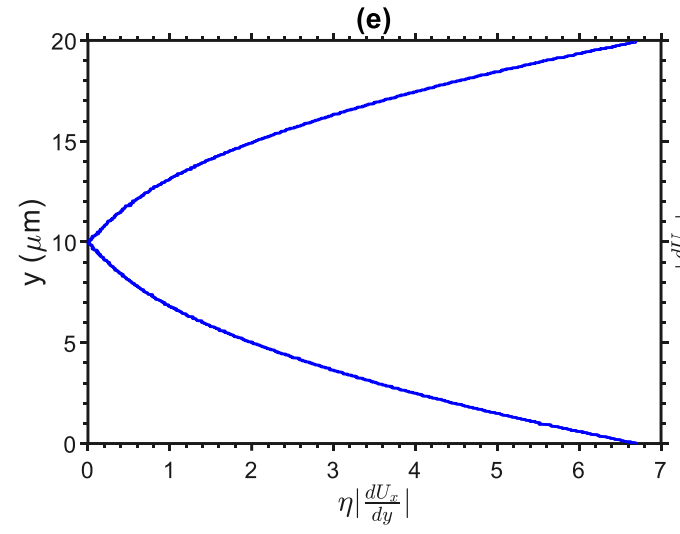

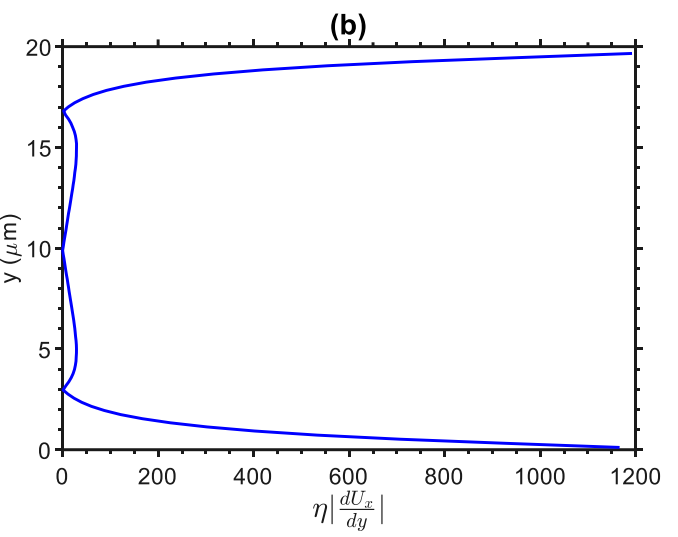

(d)

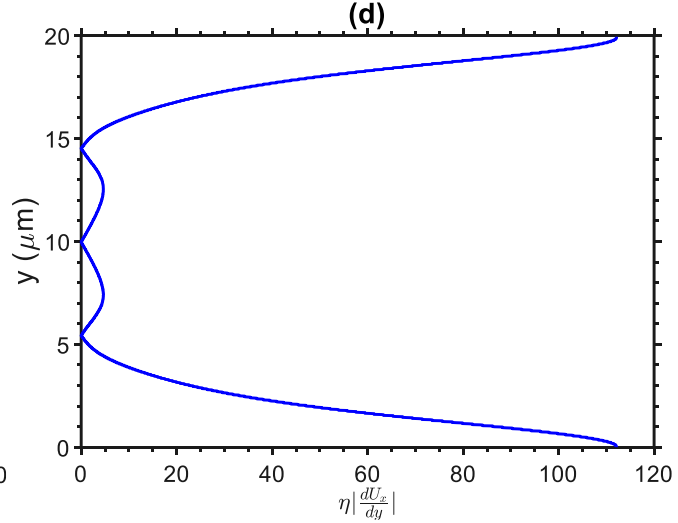

(f)

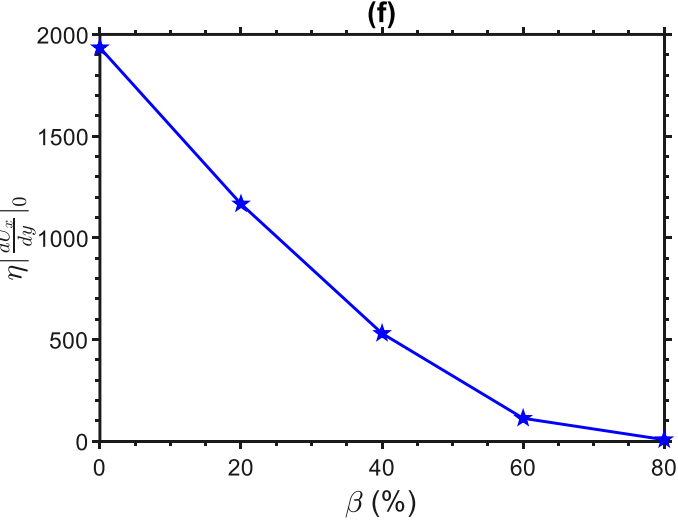

Figure 8.7: Shear stress ahead of the flame front: $\beta=0$ (a) $20 \%$ (b) $40 \%$ (c) $60 \%$ (d) $80 \%$ (e) at wall $y=0$ (f).

At highly diluted case $\beta=80 \%$, the flame shape is convex and the tip points towards the unburned matter (fuel mixture). In the flow field ahead of the flame, the flame dynamics appears to distort the flow. As observed in Fig. 8.6 - 8.7 for a streamwise velocity component and the corresponding shear stresses just ahead of the flame. This velocity profile indicates a strong interaction between the flow and flame dynamics. The streamwise velocity components decrease with dilution rates and in the $\beta<80 \%$ cases, the lip of the flame corresponds to a decline in the flow velocity. The streamwise velocity is scaled with the planar flame speeds to properly resolve 
the shape of the highly diluted cases. More so, the velocity profiles indicate that the shear layer in the cases $\beta<80 \%$ is very thin and with increasing dilution the shear layer thickness increases. Therefore, the length of the shear layer increases with dilutions and for $\beta=80 \%$, the shear layer grows into the core of the flow/channel. To further investigate the shear thinning phenomenon, the shear stresses are for cases $\beta=0 \%, 40 \%, 60 \%, 80 \%$ as shown in Figs. 8.7a -8.7 e and the wall shear stress in Figure 8.7f. In these figures the crest of the lips of the $\beta<80 \%$ flames correspond to a secondary peak shear stress. The wall shear stress is highest for $\beta=0$ at $1934.4 P a$ and decreases to $6.7 \mathrm{~Pa}$ for high dilution $\beta=80 \%$.

(a)

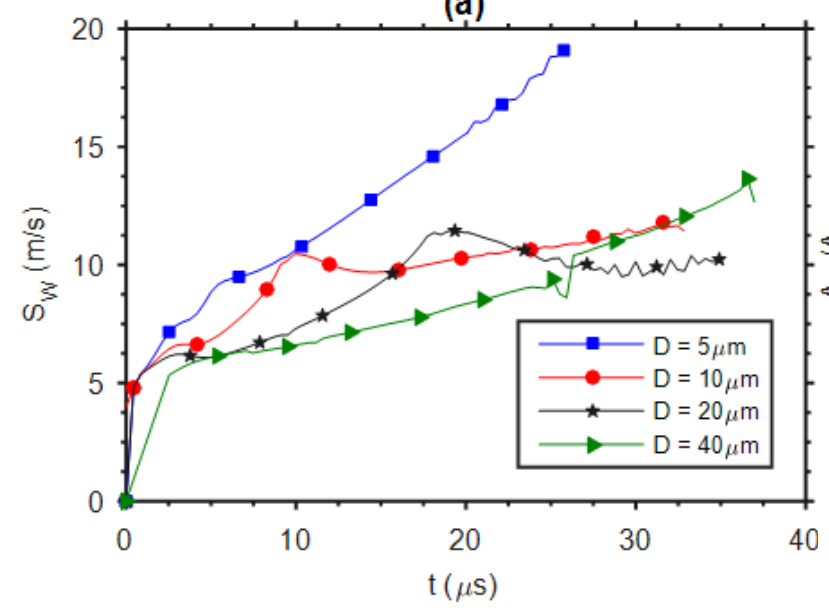

(b)

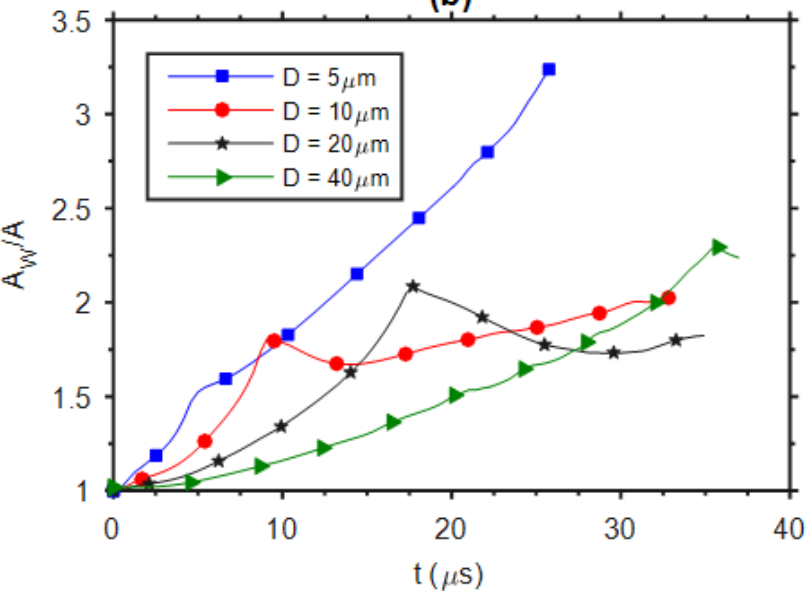

Figure 8.8: Profiles for $\beta=0$ (a) the corrugated flame velocity; (b) the scaled flame surface area.

(a)

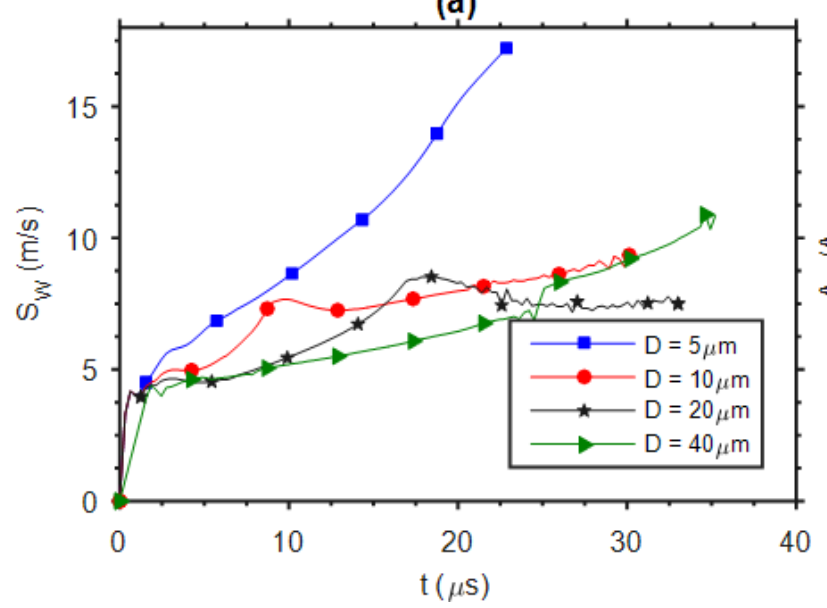

(b)

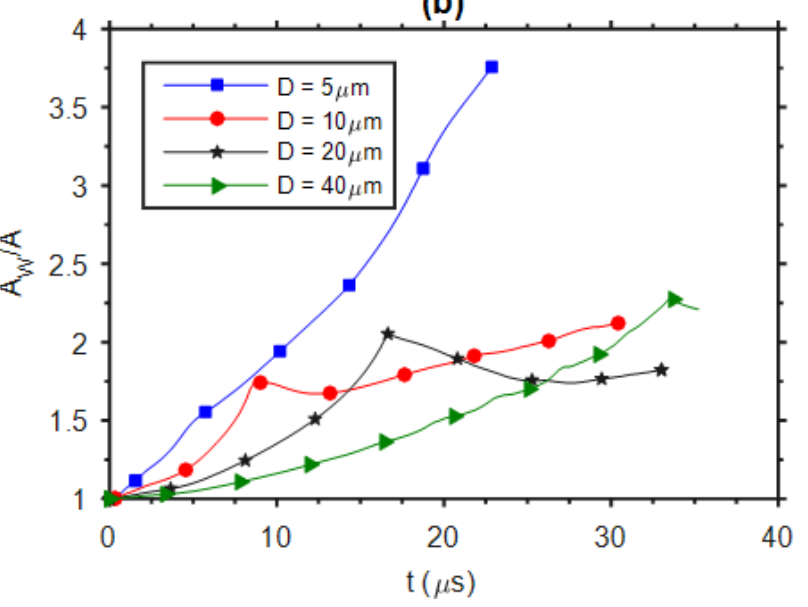

Figure 8.9: Profiles for $\beta=20 \%$ (a) the corrugated flame velocity; (b) the scaled flame surface area. 
(a)

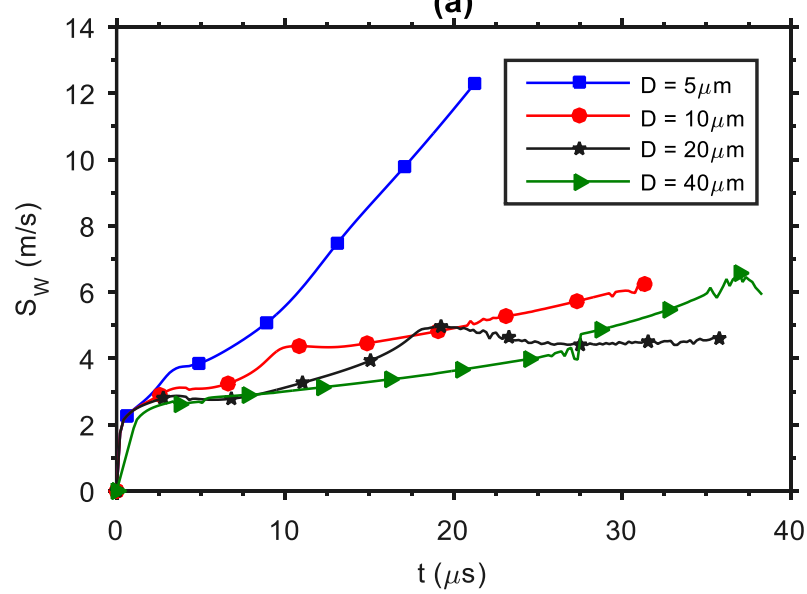

(b)

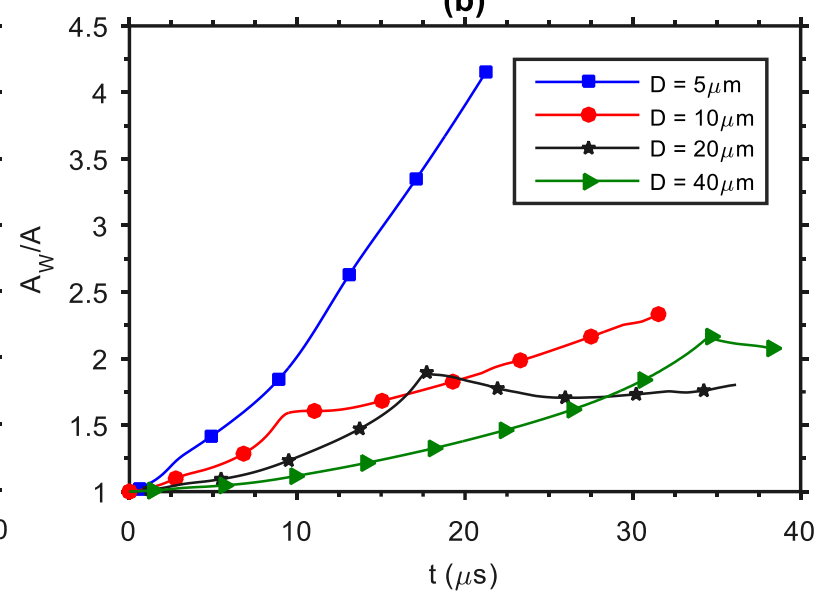

Figure 8.10: Profiles for $\beta=40 \%$ (a) the corrugated flame velocity; (b) the scaled flame surface area.

(a)

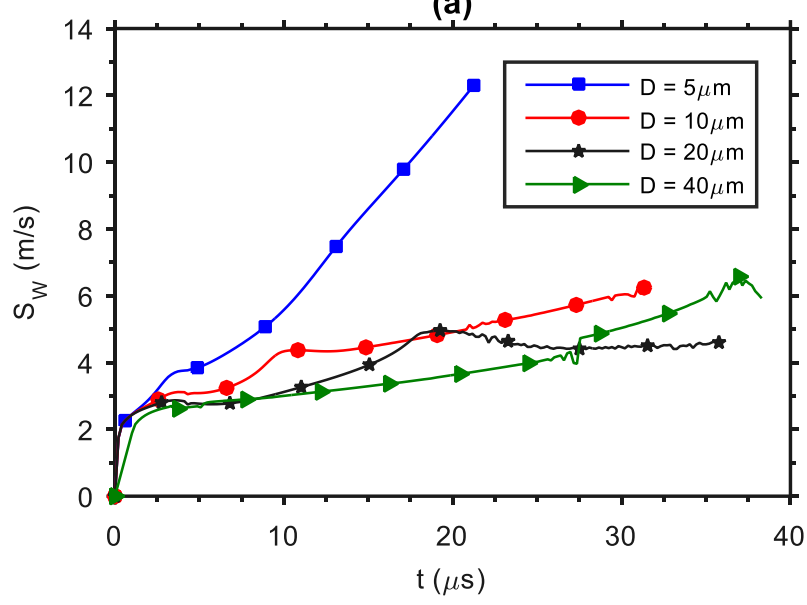

(b)

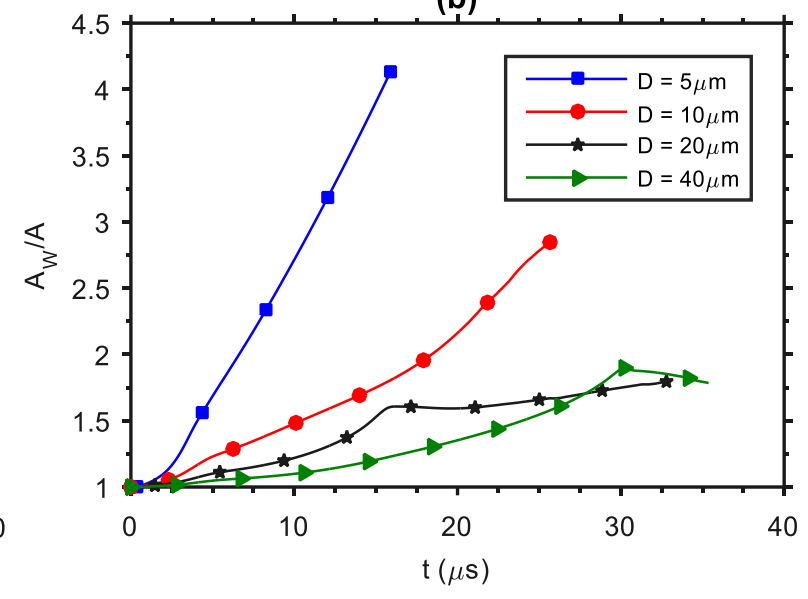

Figure 8.11: Profile for $\beta=60 \%$ (a) the corrugated flame velocity; (b) the scaled flame surface area.

(a)

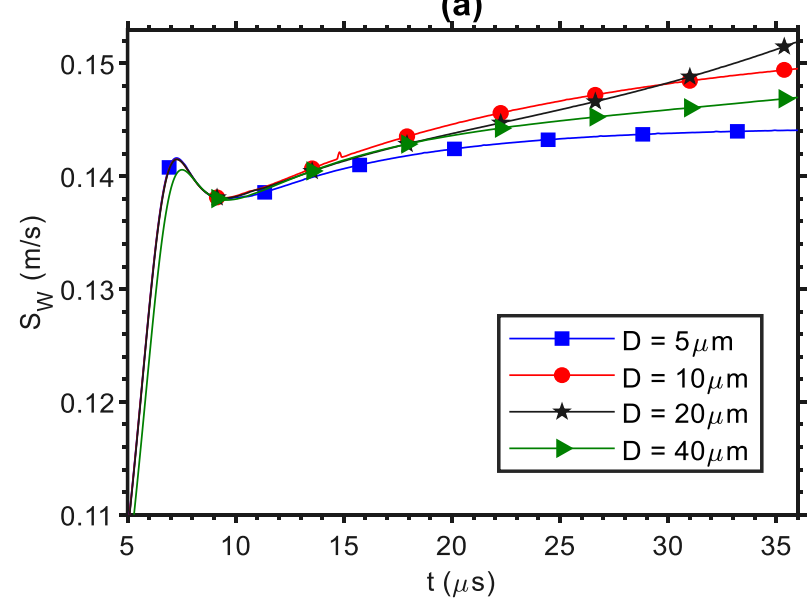

(b)

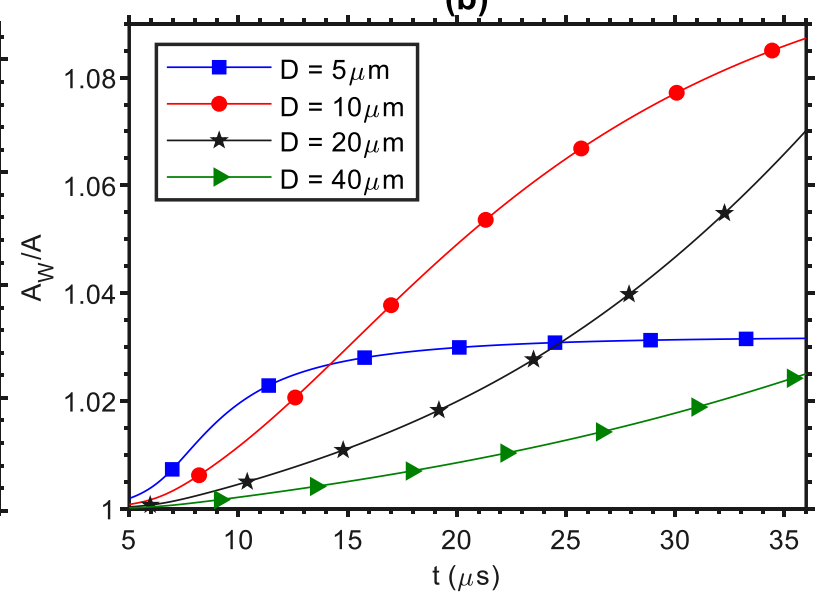

Figure 8.12: Profiles for $\beta=80 \%$ (a) the corrugated flame velocity; (b) the scaled flame surface area. 
Figures $8.8-8.12$ present the corrugated flame velocity and scaled flame area in the nonslip wall cases. The corrugated flame speed $S_{W}$ is calculated from the mass flux in the region of the flame divided by the density and the average flow velocity ahead of the flame subtracted from it. While the scaled flame area was calculated from the length of the isotherm corresponding to that having the highest reaction rates within the flame divided by the width of the channel $D$. Both variables correlate well with each other. The figures indicate that the flame front accelerates when it interacts with non-slip wall effect and the acceleration rates decrease with increasing dilution rates $\beta$. More so, the influence of hydraulic resistance relating to channel width $D$ is apparent in all dilution rates. As observed, the corrugated flame acceleration rates decrease with increase in the channel width $D$ for all $\beta<80 \%$ considered. The peak flame acceleration occurs at $D=5 \mu \mathrm{m}$ and decreases as the $D$ increases. In all the $\beta<80 \%$ cases, the trend of these accelerating flame appears to occur in two phases; first a quick exponential acceleration which subsequently changes to a flame propagating quasi-linearly in time. The duration of the initial exponential acceleration also increases with $D$. While the channel width $D$ effects are quite strong for $\beta<80 \%$, at high dilution $\beta=80 \%$ the effects of channel widths on the corrugated flame velocity are almost negligible. However, with $D=5 \mu m$, the flame shows no apparent acceleration, this length scale is comparable to the flame thickness, and as a result the flame appears to be choked in the narrow passage. Overall, the $\beta=80 \%$ flames do not indicate any significant effect of the channel width sizes on its propagation rates. Since the flame thickness grows considerably large at $\beta=80 \%$ and the wall shear stress diminishes significantly, such trend of propagation is feasible.

(a)

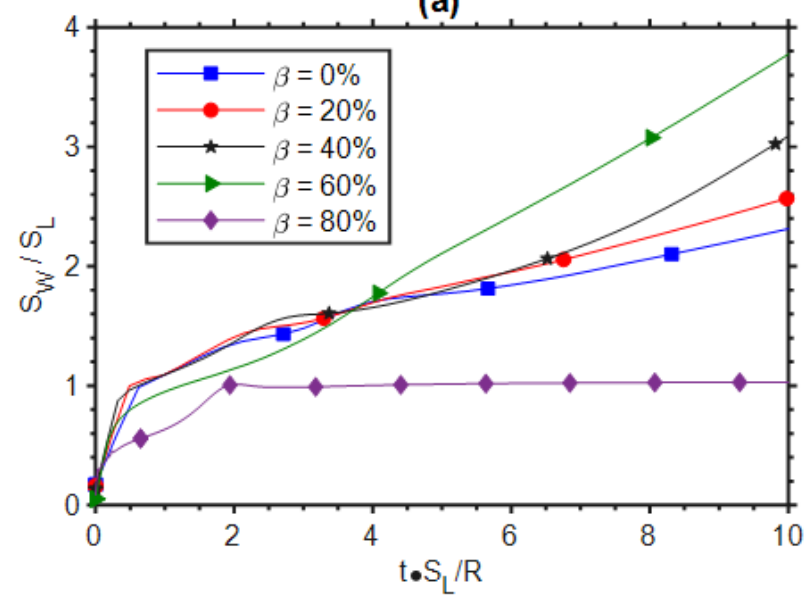

(b)

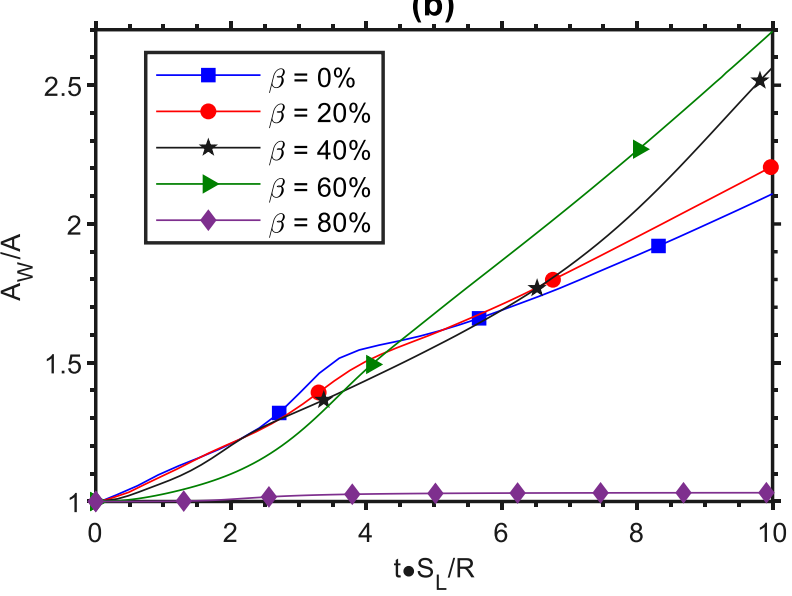

Figure 8.13: Profiles for $D=5 \mu m$ (a) the corrugated flame velocity; (b) the scaled flame surface area. 
(a)

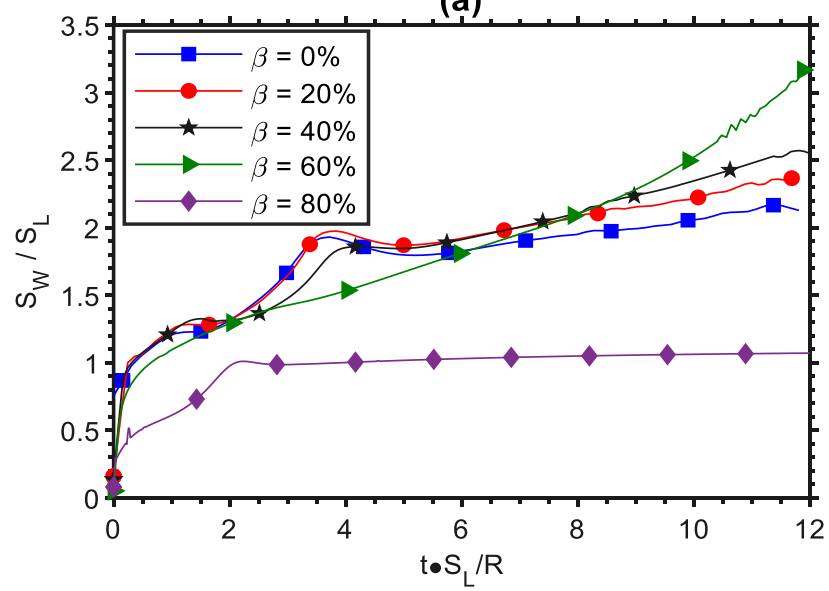

(b)

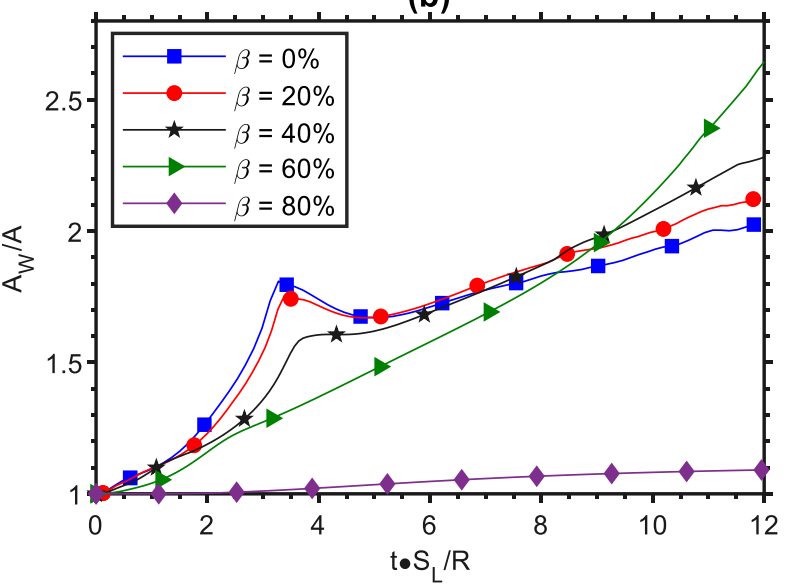

Figure 8.14: Profiles for $D=10 \mu m$ (a) corrugated flame velocity (b) scaled flame surface area

(a)

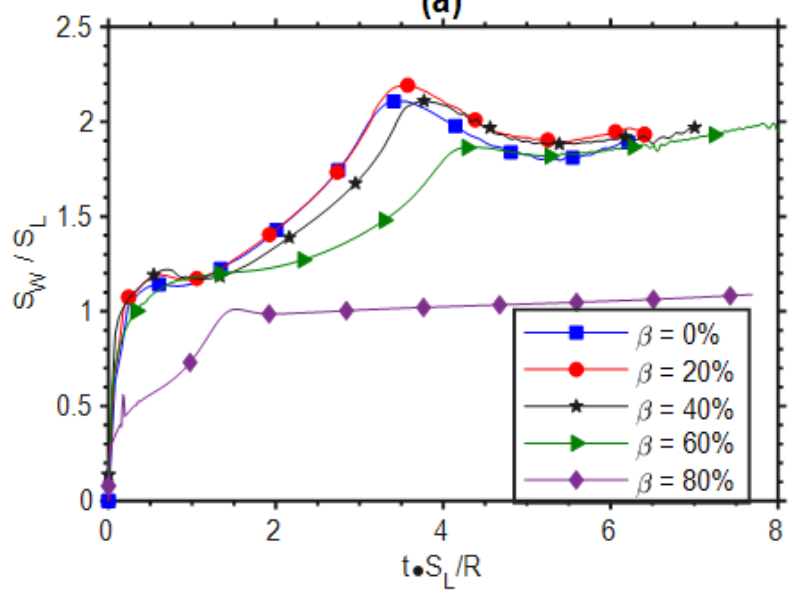

(b)

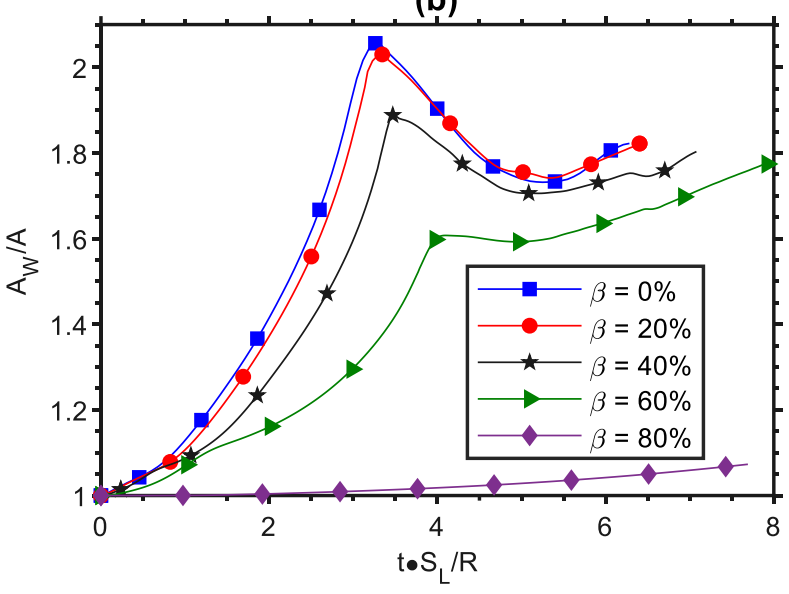

Figure 8.15: Profiles for $D=20 \mu m$ (a) the corrugated flame velocity; (b) the scaled flame surface area.

(a)

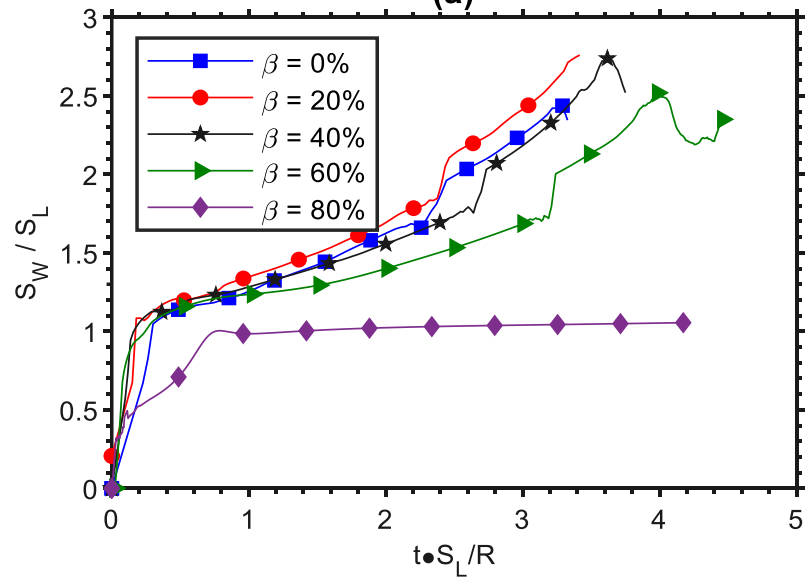

(b)

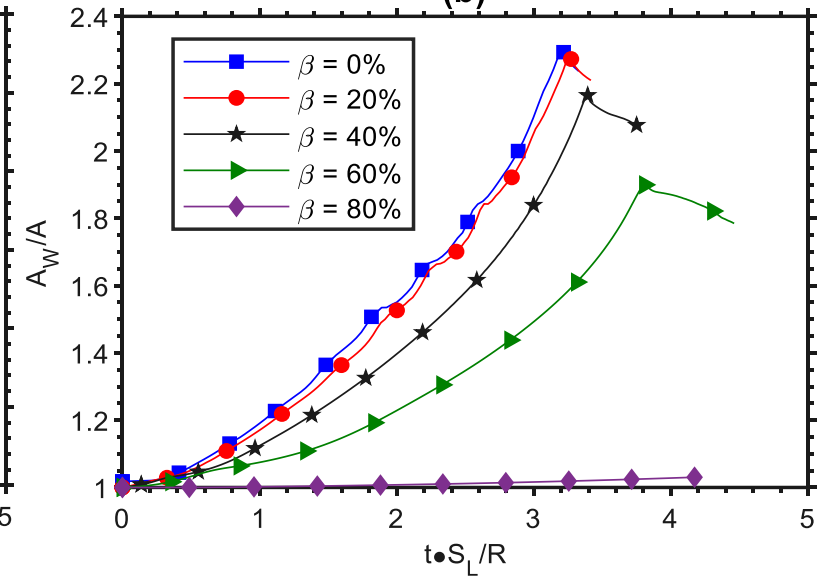

Figure 8.16: Profiles for $D=40 \mu m$ (a) the corrugated flame velocity; (b) the scaled flame surface area.

Propagation of a corrugated flame with respect to the dilution rates in each channel width sizes are presented in Figs. $8.13-8.16$, with the corrugated flame velocity scaled with the corresponding 
planar flame speeds. Also, the time is scaled with planar flame speeds and the half width of the channel $R$. For $D=5,10 \mu m$, the scaled acceleration rate increases as dilution rate increases when $\beta<80 \%$. Also, the initial exponential acceleration rates are quite diminished for these width sizes. At larger width, $D=20,40 \mu \mathrm{m}$ the scaled corrugated velocity is mostly exponential, and the exponential acceleration rate decreases with increase in the dilution rates. At the end of the exponential acceleration, the flame propagates with a near linear trend, with the velocity showing lesser dependence on the dilution rates. At larger channel width, it is expected that a flame becomes unstable due to hydrodynamic instability which is apparent in the trend at $D=40 \mu \mathrm{m}$. As such the linear flame propagation in the smaller $D$ are not apparent with larger width sizes. Overall, at high dilution of $\beta=80 \%$ the flame propagation is quite slow, limited by the flame thickness, the dilution rates and absence of significant shear stress. In the presence of heat loss from the walls and large dilution $\beta>80 \%$, it is expected that such flame might experience deceleration due to near wall quenching from heat losses to the walls. The scaled flame area also predicts similar trends as the scaled flame velocity and provides validations for the results of the scaled corrugated velocity trends.

Furthermore, the flame shapes at varying times as the flame moves through the channel are extracted. These shapes represent the isotherms corresponding to the reactive parts of the flame. Each figure tracks a flame front through the channels. It is seen that the corrugated flame thickness increases with $\beta$. As seen in Figures 8.17a -8.17 , numbers (1) - (4) represent various times corresponding to the flame positions. For Figure 8.17a, time sequence (1), (2), (3), (4) represent $3.76,11.56,19.35,26.75 \mu$ s respectively. These series of shape formed at the various times provide further insights into the flame propagating trends. As observed, for Figure 8.17a - c, at (1), the flame front shows three distinct forms, consisting of two crests and a trough at the central part of the channel. As the flame progresses to time (2), the trough has diminished, and the flame appears to have two distinct lips (tulip). These trends are consistent in cases of $\beta=0,40,60 \%$. Apparently, the areas of the flame having three parts would be larger and possess faster flame propagation rates, which explain the reason for the initial quick exponential acceleration stage. The three parts form correlates to the exponential regime of the propagation. The case of $\beta=$ $80 \%$ shows no appearance of three-part or two-part (tulip) flame. 

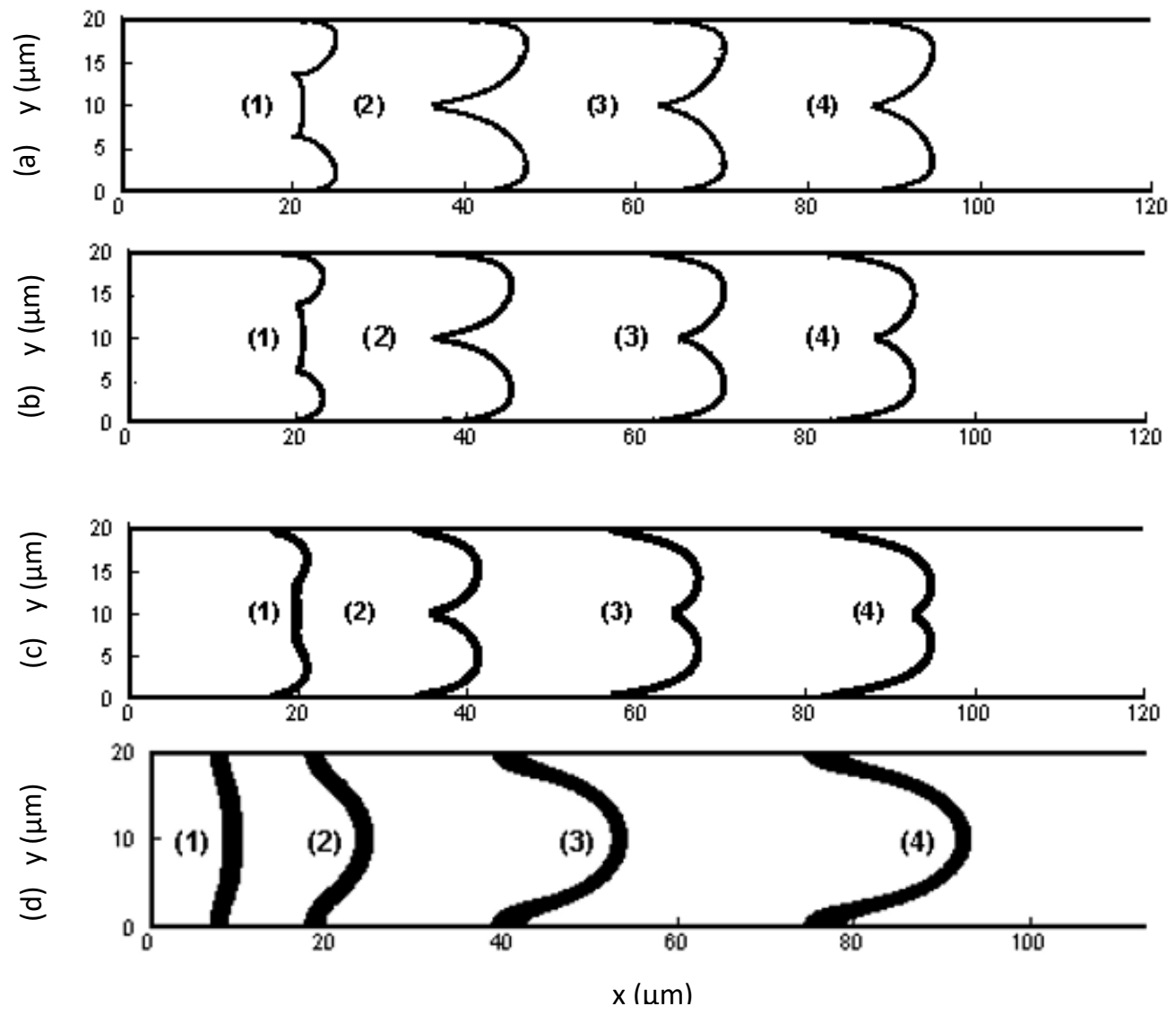

Figure 8.17: Flame shape variation for $D=20 \mu \mathrm{m}$ (a) $\beta=0 \%$ (b) $\beta=40 \%$ (c) $\beta=60 \%$ (d) $\beta=80 \%$.

Obviously, the highly diluted case has a relatively large flame thickness which provides high stability of the flame structure and prevents it from wrinkling in many forms as observed for the low or non-diluted cases. The thermal expansion of the burning matters impacts on the flame structure uniformly and the non-slip nature of the channel causes the flame to acquire the convex form. Similarly, the time sequence in Fig. 8.17b correlates with 9.63,16.98, 25.2,33.24 $\mu \mathrm{s}$ for (1) - (4). Moreover, in Fig. 8.17c, they correlate with 11.03, 19.25, 27.55, $35.7 \mu$ s. And for $\beta=80 \%$, Fig. 8.17d, (1), (2), (3), (4) are related to 23.7, 56.2, 102.9, $147.7 \mu$ s. Overall, this correlates with the flame acceleration scenario in terms of the flame shapes evolution and it gives better understanding to the evolution of supercritical flames in semi-open channels under various conditions. In Fig. 8.18, the flame shapes are shown for width $D=40 \mu \mathrm{m}$ and $\beta=0,60 \%$. These figures show similar shapes as in a narrow channel. However, the three-part flame shapes persist for long duration in both dilution rates. These also replicate the prolonged exponential propagation phases revealed in Fig. 8.16. 

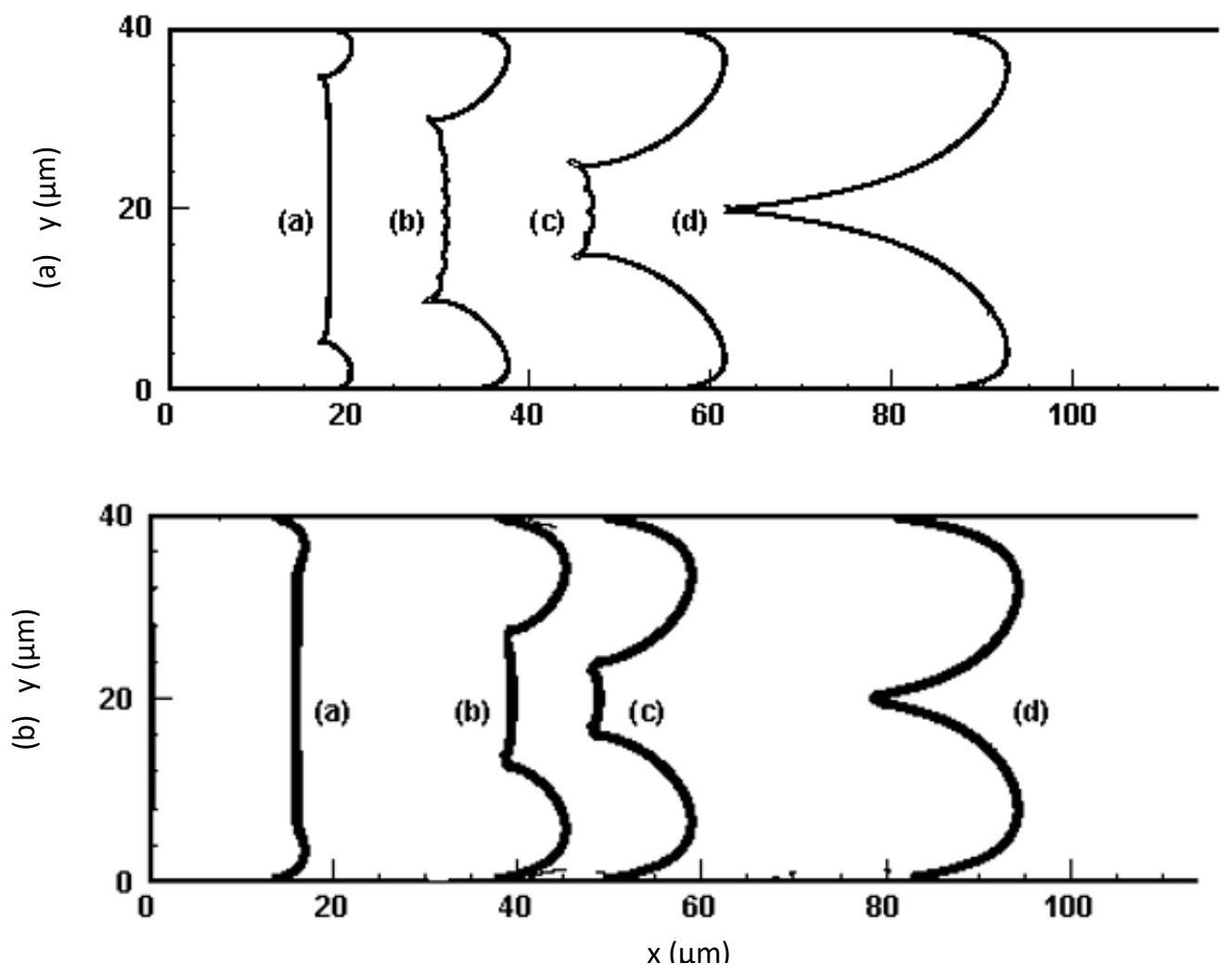

Figure 8.18: Flame shape variation in for $D=40 \mu \mathrm{m}$ (a) $\beta=0 \%$ (b) $\beta=60 \%$

\subsection{Estimation of the Markstein Length/Number}

Finally, simulations of spherically expanding supercritical methane flames for dilution rates $\beta=0,40 \%, 60 \%, 80 \%$ for $T_{f}=800 K, P_{f}=300 \mathrm{bar}$ was conducted solely to calculate the Markstein lengths and numbers at these conditions. The switch in flame form occurring with dilution rates prompted this investigation. The Markstein number $M k$ is a dimensionless value which describes how the changes in the flame surface curvature and the corresponding stretched flame velocity are influenced by the local heat release [61].

$$
M k=L_{b} / \delta_{L},
$$

where $L_{b}$ represents the Markstein length with respect to the burnt matter and it quantifies the influence of the flame stretch on the laminar flame velocity. For a moderately stretched flame, the relationship between the stretched flame velocity and the stretch rate can be considered linear [62]

$$
S_{b}=S_{b}^{0}-L_{b} K,
$$

where $S_{b}^{0}$ is the unstretched laminar flame speed with respect to the burnt matter and $K$ signifies the flame stretch rate [63]. The value $K$ is defined for a spherically propagating flame as 


$$
\mathrm{K}=\frac{2}{\mathrm{R}_{\mathrm{f}}} \frac{\mathrm{d} r_{f}}{\mathrm{dt}},
$$

where $r_{f}$ represents the flame radius which is a function of time. Similarly, the stretched flame velocity is defined as

$$
S_{b}=\frac{\mathrm{d} r_{f}}{\mathrm{dt}}
$$

To extract the Markstein length from Equation 8.4, a linear regression of the flame stretch rate with the stretched flame velocity is performed as seen in Fig. 8.19 for each of the dilution rates. The Markstein lengths shown in Fig. 8.20a is positive for the cases $\beta<80 \%$ and negative when $\beta=80 \%$. Similarly, the Markstein numbers $M k$ shown in Fig. 8.20b decreases from 1.50 to -0.37 as the dilution rate rises from $\beta=0$ to $80 \%$.

(a)

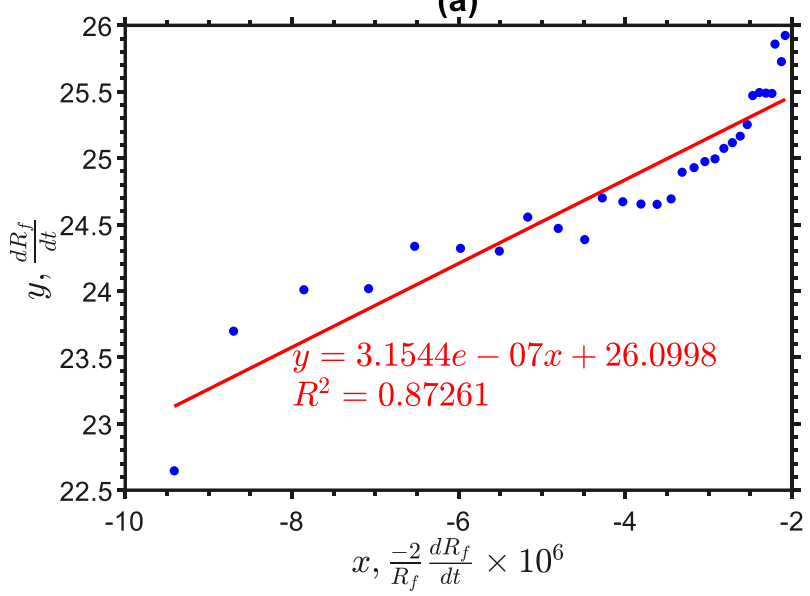

(c)

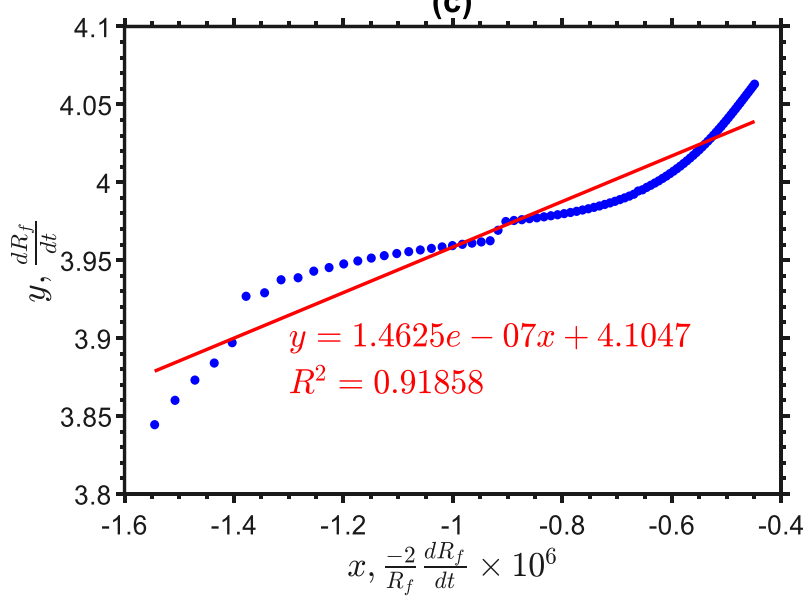

(b)

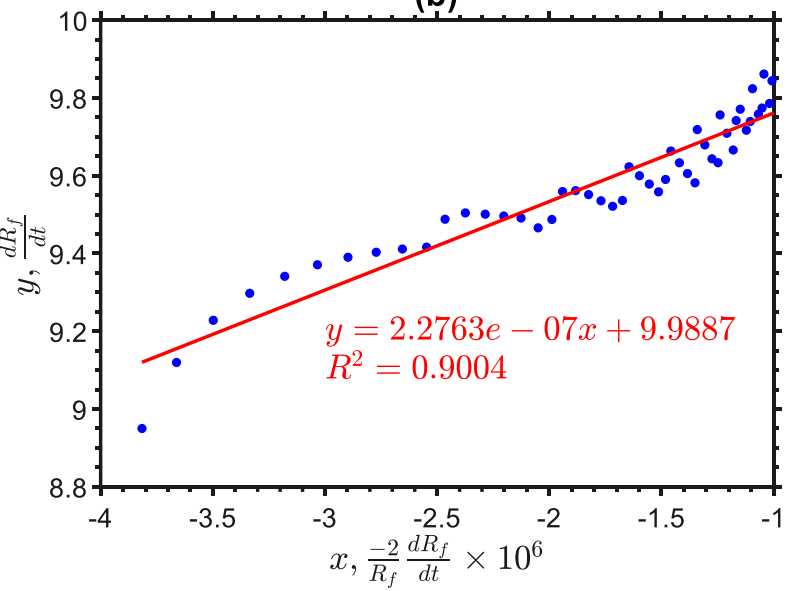

(d)

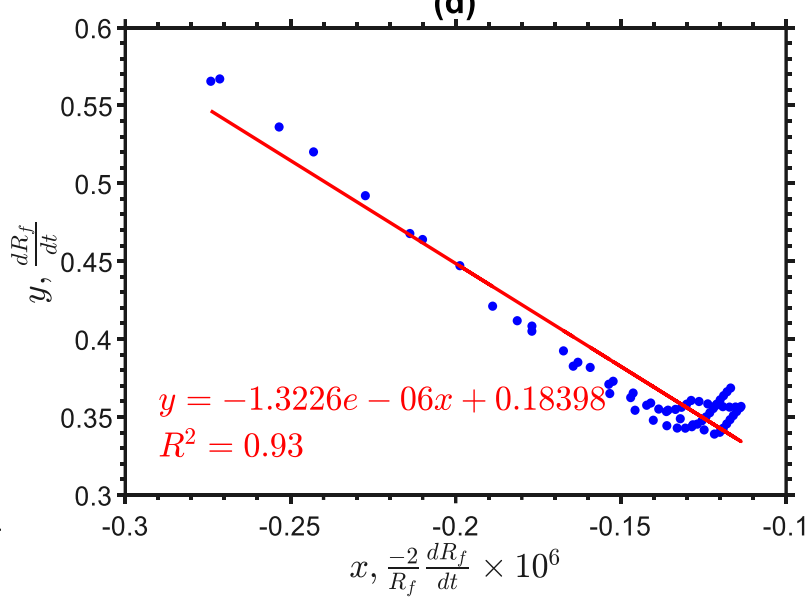

Figure 8.19: Markstein length predictions (a) $\beta=0 \%$ (b) $\beta=40 \%$ (c) $\beta=60 \%$ (d) $\beta=8$ 
(a)

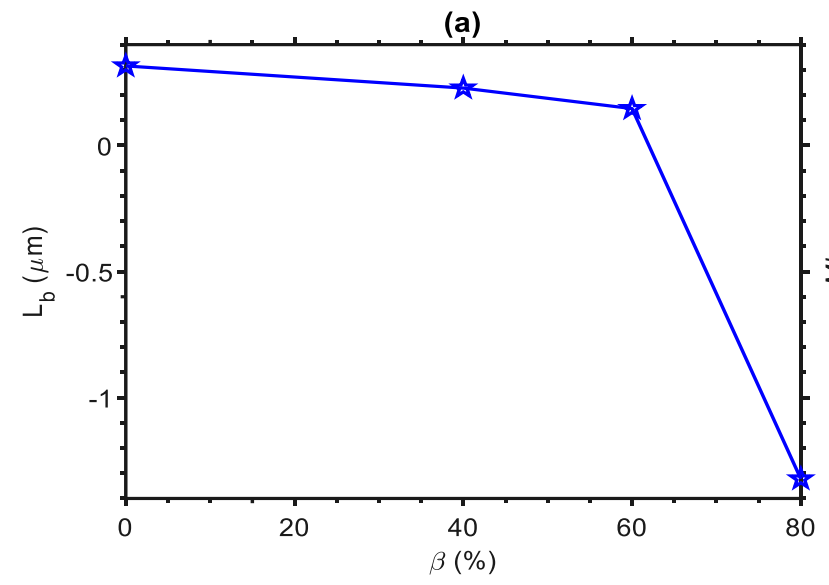

(b)

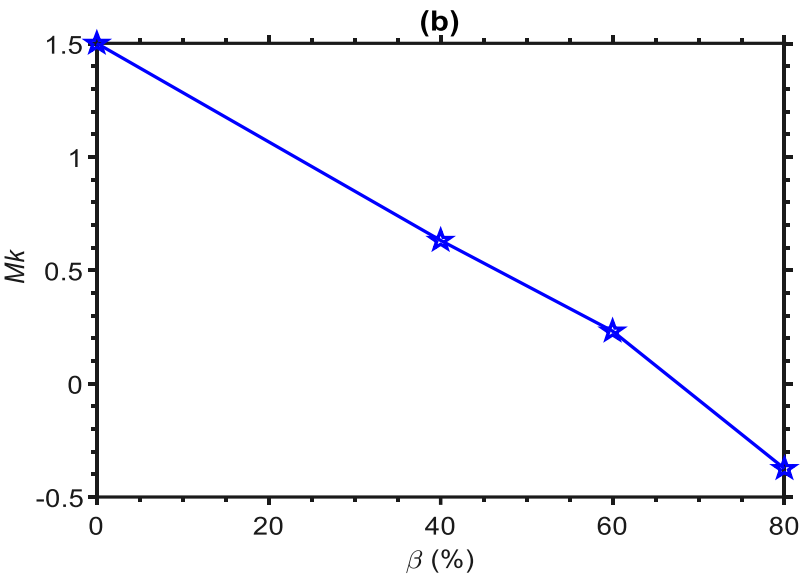

Figure 8.20: Dilution effect on (a) Markstein length (b) Markstein number 


\section{Supercritical Flames in a Semi-Open Obstructed Channel}

\subsection{Flame Morphology and Propagation}

Figures $9.1-9.3$ present the contour plots of the temperature, vorticity magnitude, and shock interface at the early and later period of the flame acceleration for $\alpha=1 / 3,1 / 2,2 / 3$ and $\beta=0$. These figures present the morphology of the supercritical oxy-methane flame in the three blockage ratios. The corresponding propagation trends are shown in Figure 9.4. As the front propagates in time, initially due to thermal expansion at the core of the channel (unobstructed parts), it leaves behind pockets of unburnt gases, as seen in the temperature snap shots. The eventual burning of the gases in these pockets generates more expansion of gases into the core, leading to large scale flame wrinkling and jet flow development which increases the flame velocity. The temperature snapshot gives an indication of the flame shape. The flame generally begins with a concave "tulip" structure and develops further in time for low to moderate blockage ratios $\alpha=1 / 3,1 / 2$. The vorticity magnitude and shock interfaces provide further details on these trends. For low and moderate blockage ratios $\alpha=1 / 3,1 / 2$ the pockets ahead of the flame front are dominated by vortices which grow in magnitude with time and with blockage ratio. Both blockage ratios show oblique shock wave ahead of the flame fronts, which is stronger with increase in blockage ratio.

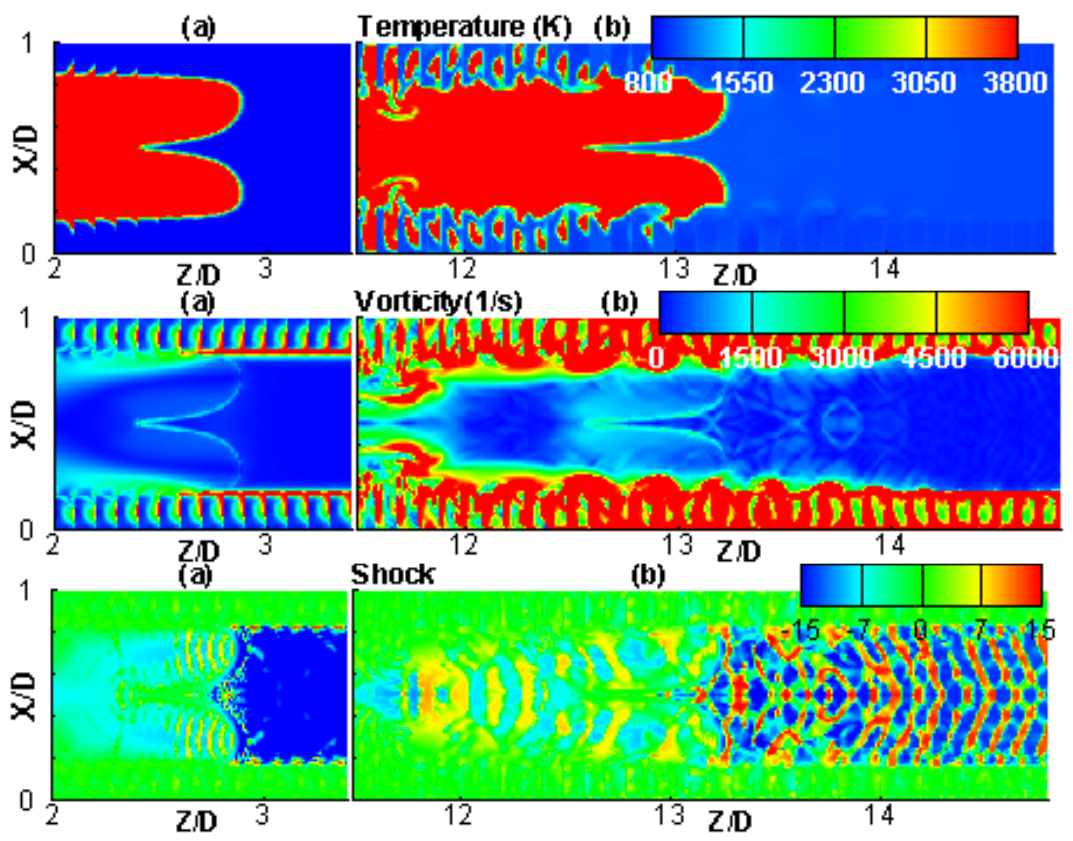

Figure 9.1: Flame snapshots for $\beta=0 \%, \alpha=1 / 3, D=12 \mu \mathrm{m}$ at times a) $t=0.35 \mu \mathrm{s}$ b) $t=0.7 \mu \mathrm{s}$ 
These vortices promote the corrugation of the flame front, leading to a more elongated tulip structure at $\alpha=1 / 2$ and stronger leading shock wave interface. At high blockage ratio $\alpha=2 / 3$ however, the initial tulip flame collapses to form a convex shape later in time, with the vortices also confined to the region behind the flame and likewise the leading shock is not apparent and restricted to the burnt region.
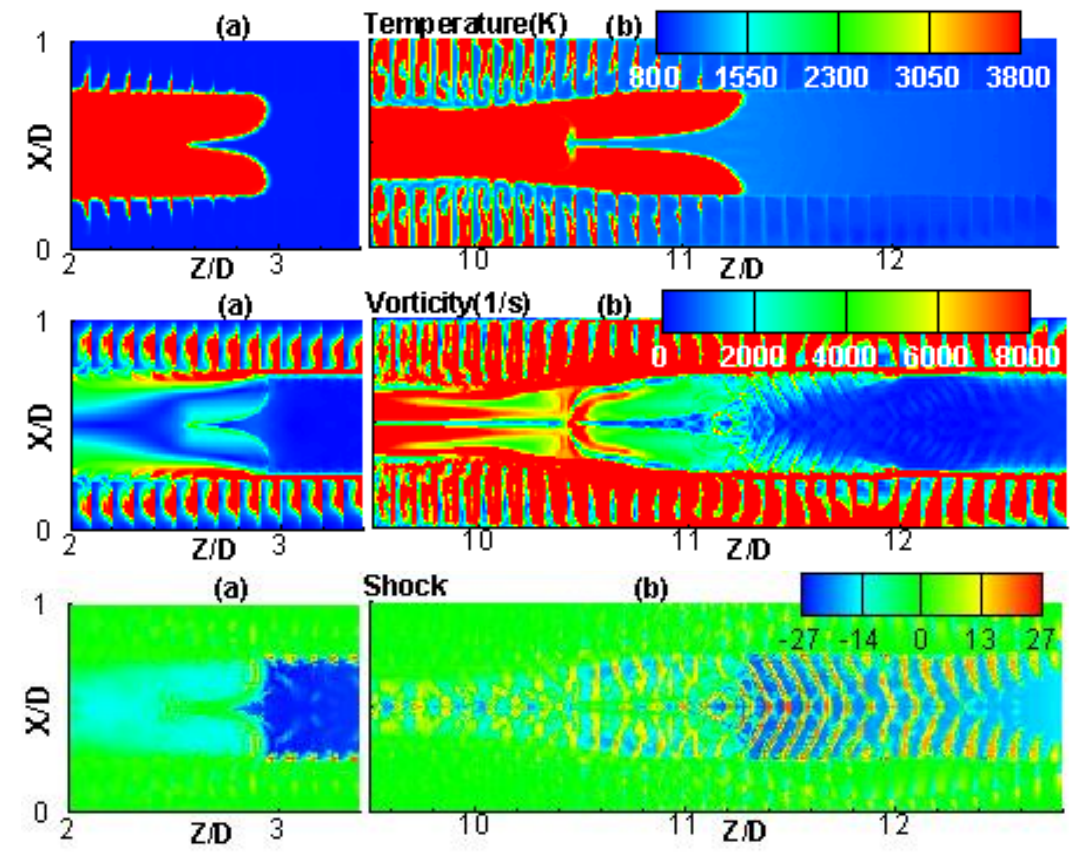

Shock

(b)

Figure 9.2: Flame snapshots for $\beta=0 \%, \alpha=1 / 2, D=12 \mu \mathrm{m}$ at times a) $t=0.25 \mu \mathrm{s}$ ) $t=0.5 \mu s$.

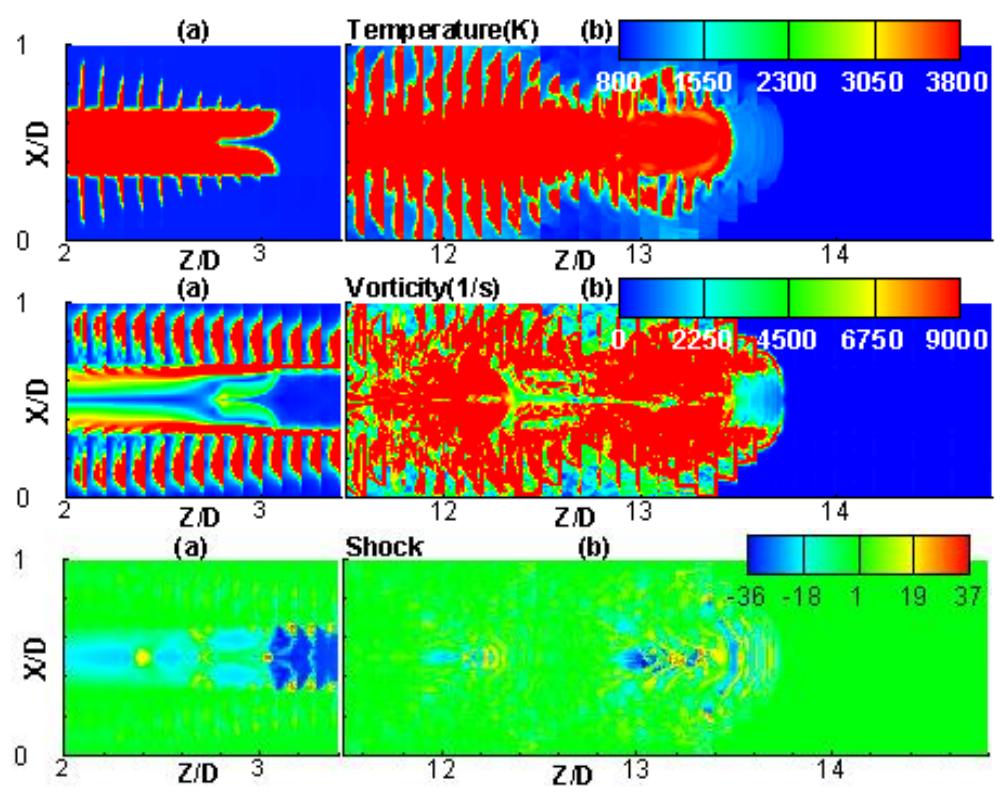

Figure 9.3: Flame snapshots for $\beta=0 \%, \alpha=2 / 3, D=12 \mu \mathrm{m}$ at times a) $t=0.35 \mu \mathrm{s}$ b) $t=0.7 \mu s$. 
(a)

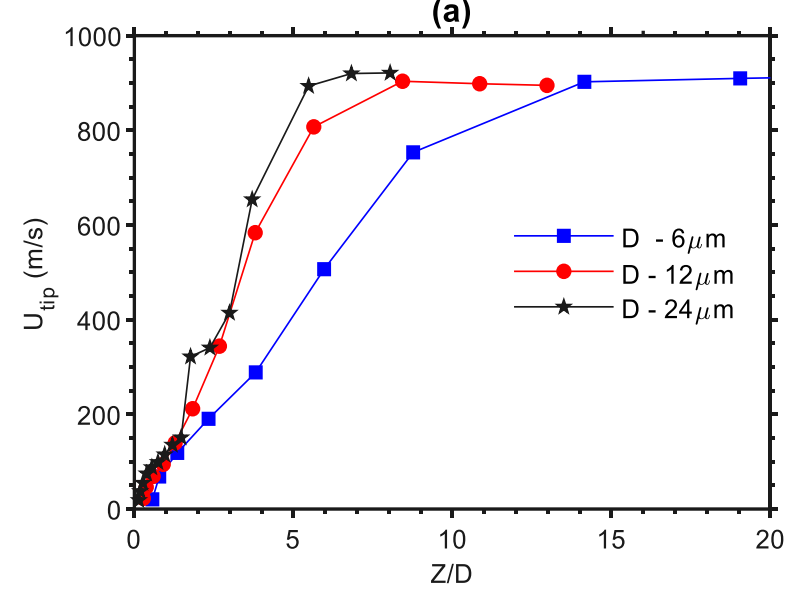

(b)

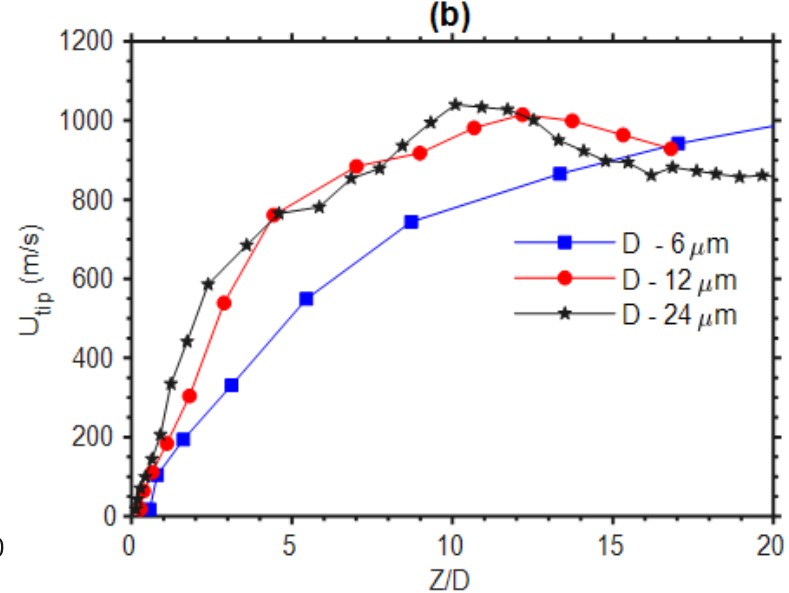

(c)

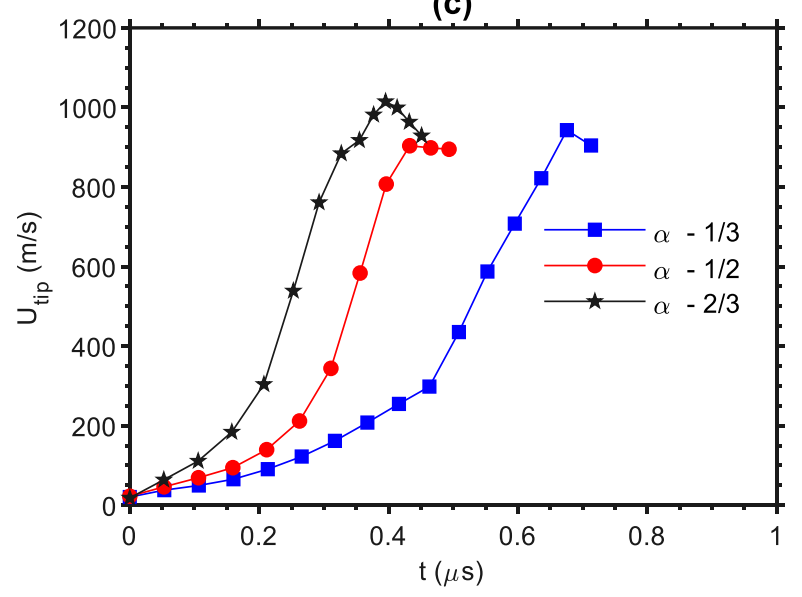

Figure 9.4: Flame tip velocity for $\beta=0 \%$, a) $\alpha=1 / 2$ b) $\alpha=2 / 3$ c) $D=12 \mu m$.

As the flame progresses in time, it saturates to constant velocity. At this late stage of the propagation, the flow in the pockets formed by the obstacles is dominated by high magnitude vortices both downstream and upstream. However, the vortices are not present in downstream of the flame when $\alpha=2 / 3$. This is quite apparent since the fast flow rate limits the formation of the vortices in the fuel mixtures ahead of the flame. Perhaps, this indicates why the initial tulip flame changes to the convex form at the saturated state. More so, the shock interface also indicates that the compression waves, i.e. shock waves, are quite weak ahead of the flame for $\alpha=2 / 3$. While, for $\alpha=1 / 3,1 / 2$ an oblique shock wave appears in the flow downstream of the flames. Figures $9.4 \mathrm{a}-\mathrm{b}$, show how the flame tip propagates along the length of the channel. As seen in Figures 9.4a, b for $\alpha=1 / 2,2 / 3$, the flame tip velocity collapses to about the same saturated (choked) velocity for $D=6,12,24 \mu \mathrm{m}$. For all the $\alpha=1 / 3,1 / 2,2 / 3$ and $\beta=0$, average flame tip velocity for the choked flames is above speed of the sound in the fuel mixture. The result of the variation of flame tip velocity with time for $\alpha=1 / 3,1 / 2,2 / 3$ and $\beta=0$ indicates that the flame 
tips propagates exponentially in time before attaining saturation, as presented in Fig. 9.4c. Likewise, the trend is also consistent with that reported by Bychkov [15,16,34], which shows an increase in the acceleration rates with increases in $\alpha$. Figures $9.5-9.7$ show the flame snapshots at dilution rates corresponding to $\beta=40 \%$ and the observed flame shapes resemble $\beta=0$ cases. However, the concavity of the front appears reduced and both the vortices and leading shock waves are moderated.

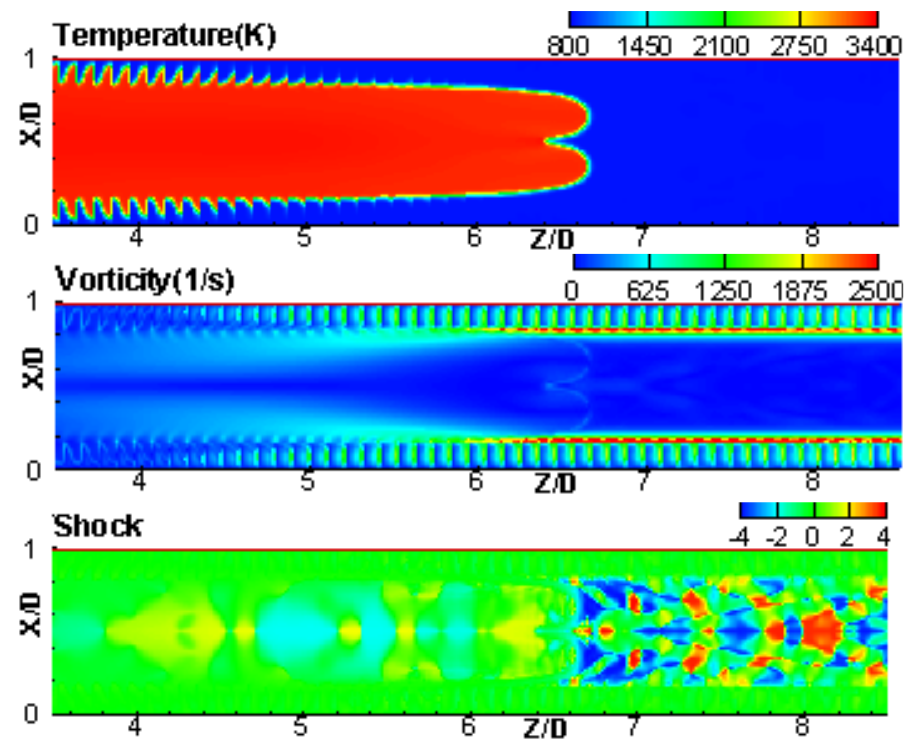

Figure 9.5: Flame snapshots for $\beta=40 \%, \alpha=1 / 3, D=12 \mu \mathrm{m}$.

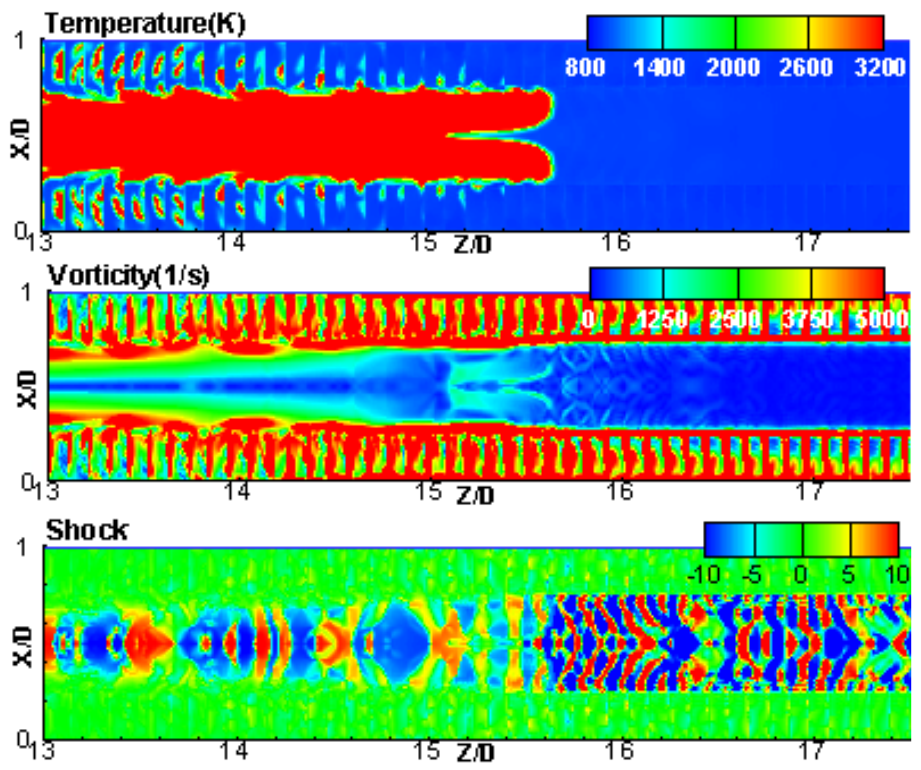

Figure 9.6: Flame snapshots for $\beta=40 \%, \alpha=1 / 2, D=12 \mu \mathrm{m}$. 


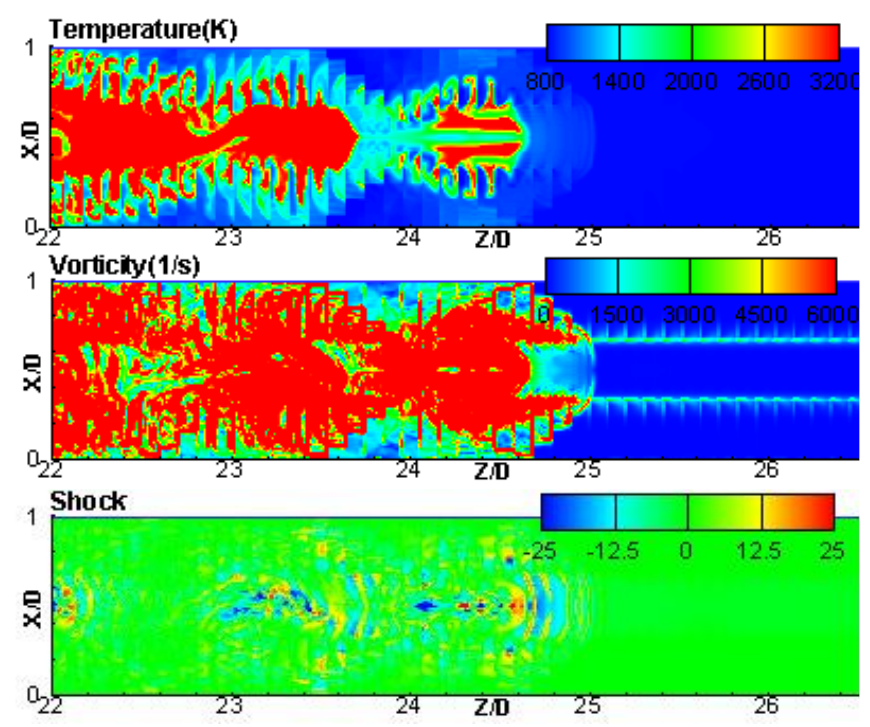

Figure 9.7: Flame snapshots for $\beta=40 \%, \alpha=2 / 3, D=12 \mu \mathrm{m}$.

However, for $\alpha=2 / 3$ the convex flame front breaks up at the saturated "choked velocity" regime. Similar, to the case of $\beta=0$ the high magnitude vortices and the shock wave for $\alpha=2 / 3$ are limited to the burnt regions. As observed in Fig. 9.8a, a choked velocity is approximately $800 \mathrm{~m} / \mathrm{s}$ compared to about $1000 \mathrm{~m} / \mathrm{s}$ for $\beta=0 \%$. More so, the exponential acceleration rates depend on the $\alpha$, such that it increases with $\alpha$.

(a)

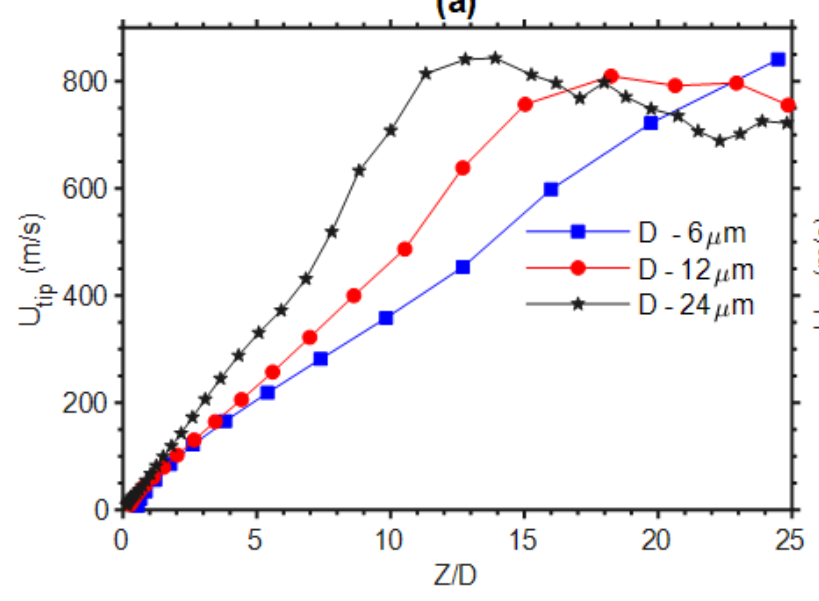

(b)

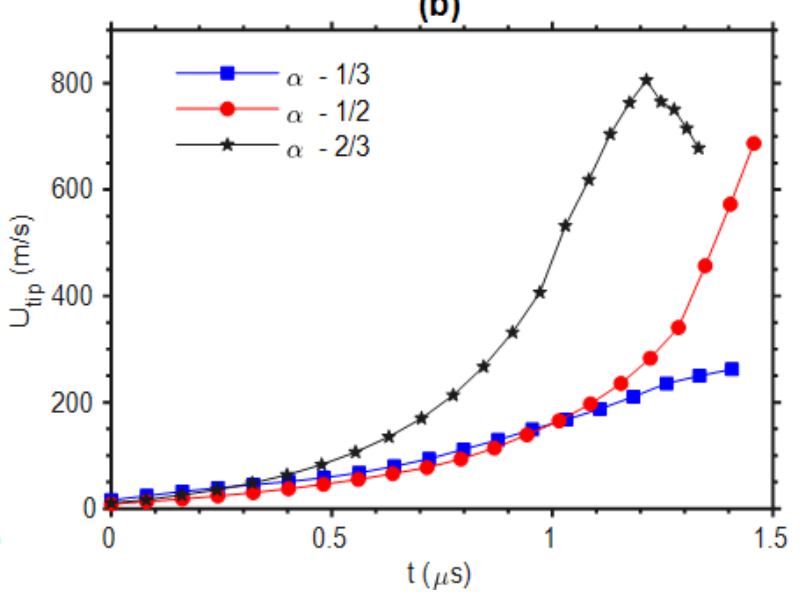

Figure 9.8: Flame tip velocity for $\beta=40 \%$, a) $\alpha=2 / 3$ b) $D=12 \mu m$.

When the dilution rate increases, the flame becomes much stable, propagates much slower and acquires a convex form. In Figs. 9.9 - 9.12, the snapshots of the flames for $\beta=60 \%$ show a largely stable flame, with vortices, developed ahead of the flame front at the edge/entrance to the pocket formed by adjacent obstacle and compression waves propagating into the fuel mixture. The plots of the flame tip velocity show that the acceleration rates become almost independent of the 
width of the channels. But, the effects of blockage ratio are most prominent and consistent with previous observation.
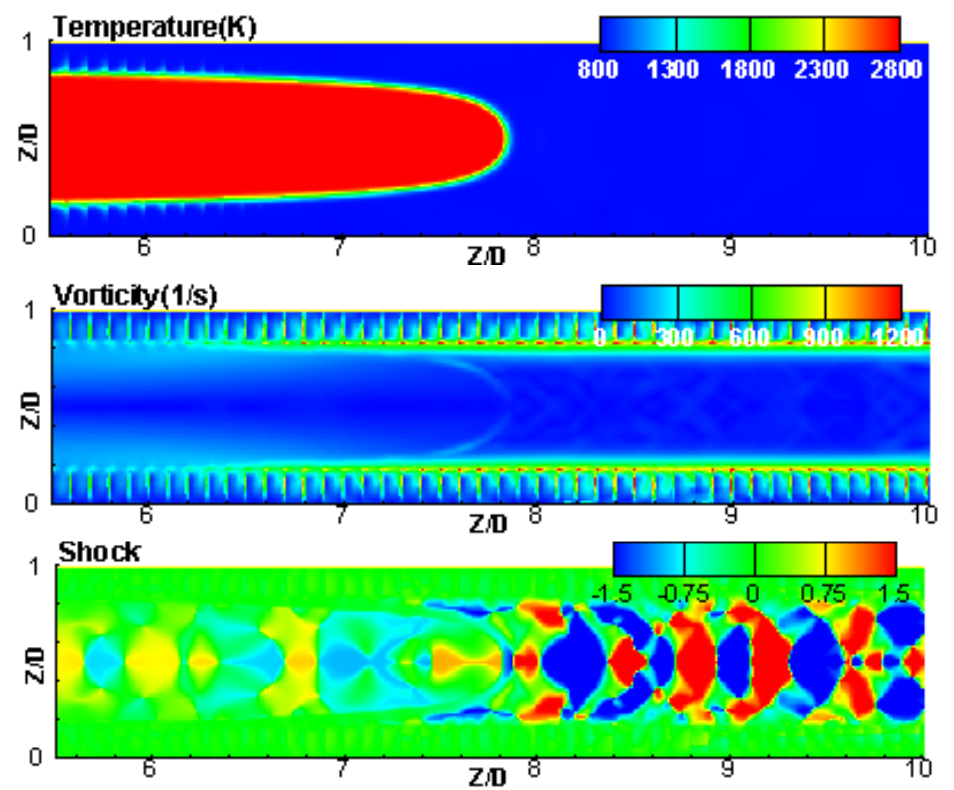

Figure 9.9: Flame snapshots for $\beta=60 \%, \alpha=1 / 3, D=12 \mu \mathrm{m}$.
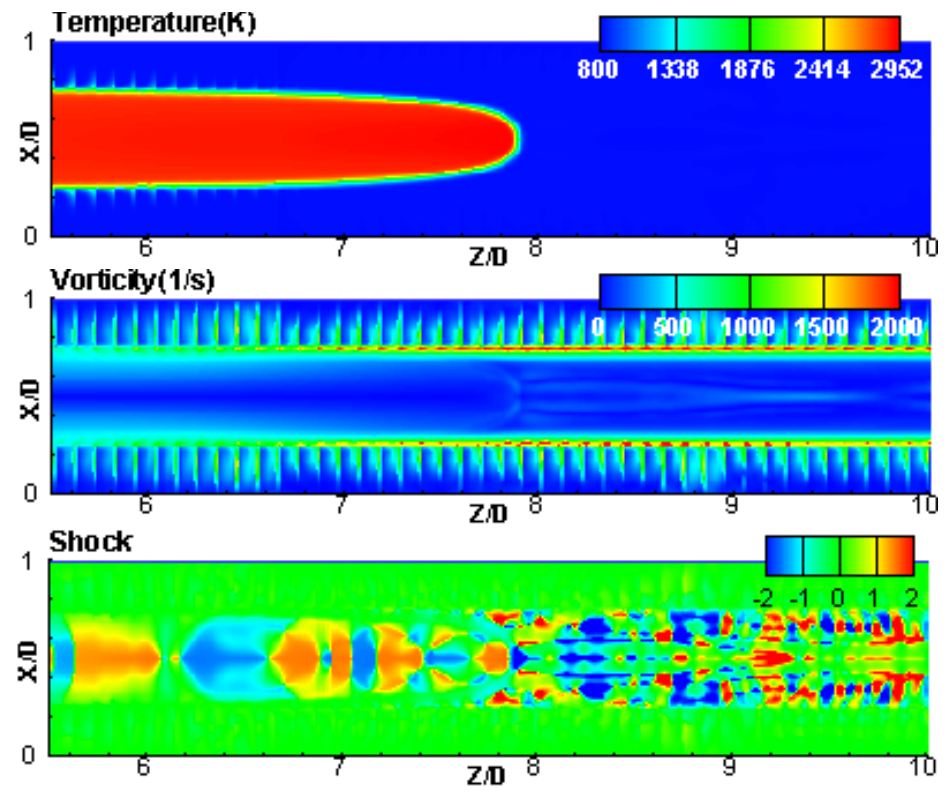

Figure 9.10: Flame snapshots for $\beta=60 \%, \alpha=1 / 2, D=12 \mu \mathrm{m}$. 


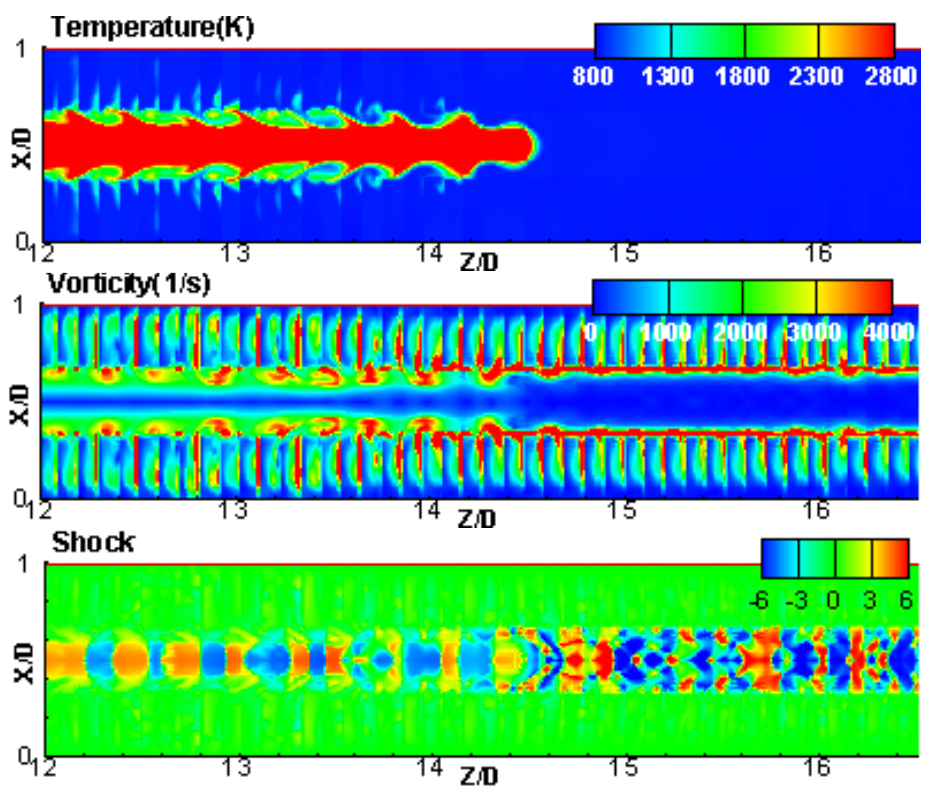

Figure 9.11: Flame snapshots for $\beta=60 \%, \alpha=2 / 3, D=12 \mu \mathrm{m}$.
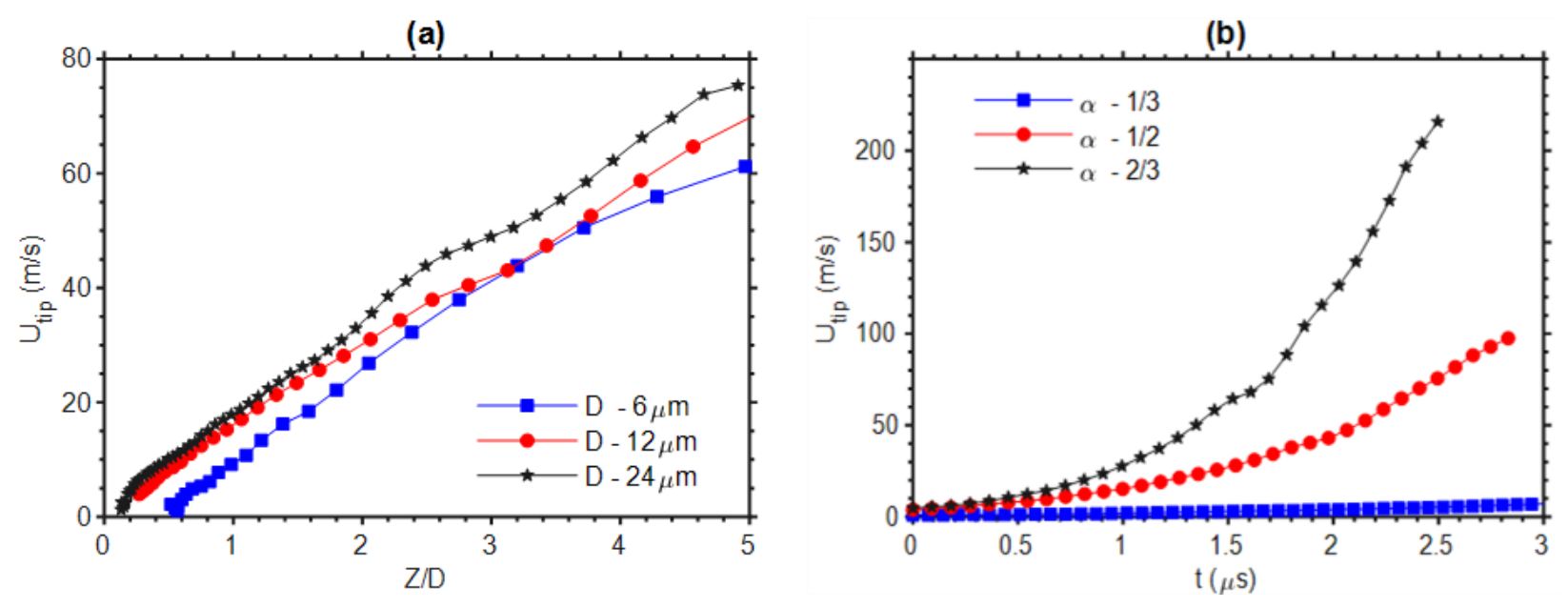

Figure 9.12: Flame tip velocity for $\beta=60 \%$, a) $\alpha=1 / 2$ b) $D=12 \mu \mathrm{m}$.

For $\beta=80 \%$, Fig. 9.15 shows an oscillating flame velocity as observed in $D=6 \mu \mathrm{m}$ channel which are caused by the hydraulic constraints imposed by the smaller channel width on the highly diluted flame which is a highly thickened flame propagating through a narrow channel with $D \sim$ $L_{f}$. Moreover, for wider channel width, the resistances are overcome, and the flame undergo accelerating trend. The flame snapshots in Figs. $9.13-9.14$, show the flames at relatively wider channel $D=24 \mu \mathrm{m}$ with thickened front. Overall, the flame shape is quite convex, and the flames propagates moderately. 

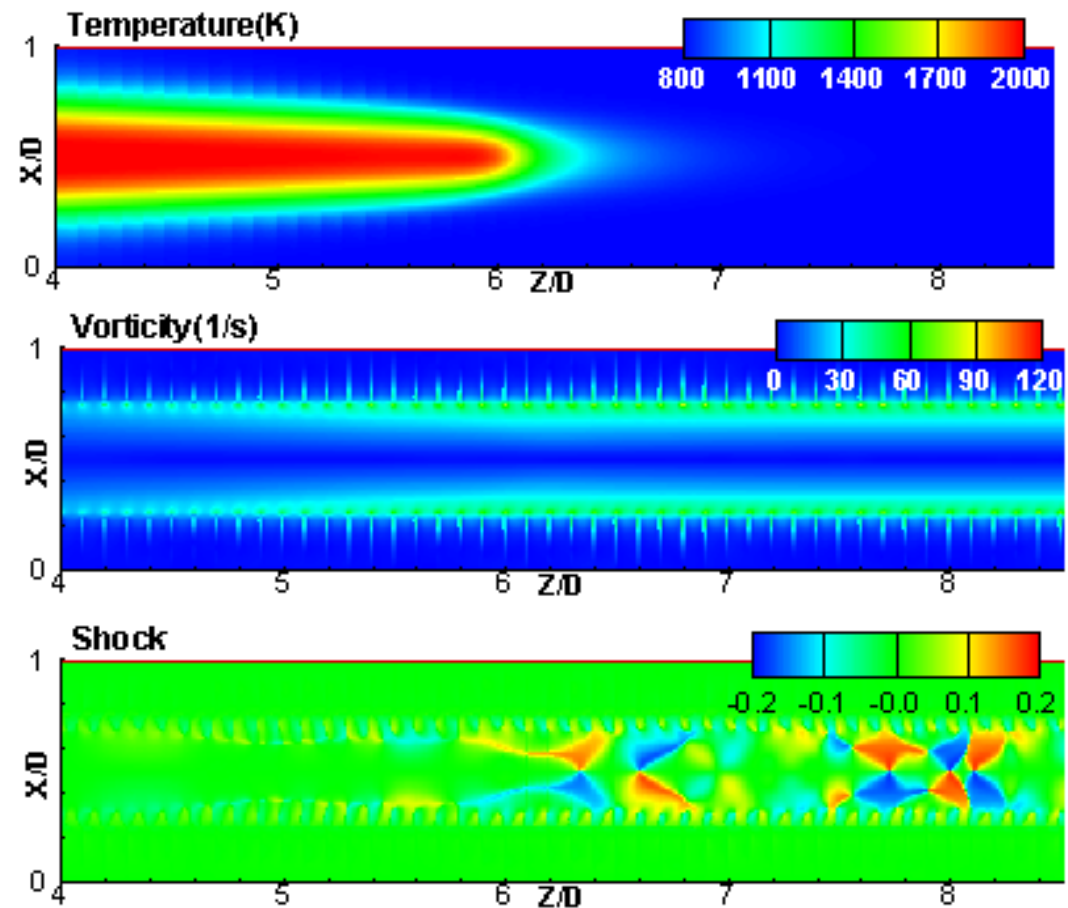

Figure 9.13: Flame snapshots for $\beta=80 \%, \alpha=1 / 2, D=12 \mu \mathrm{m}$.
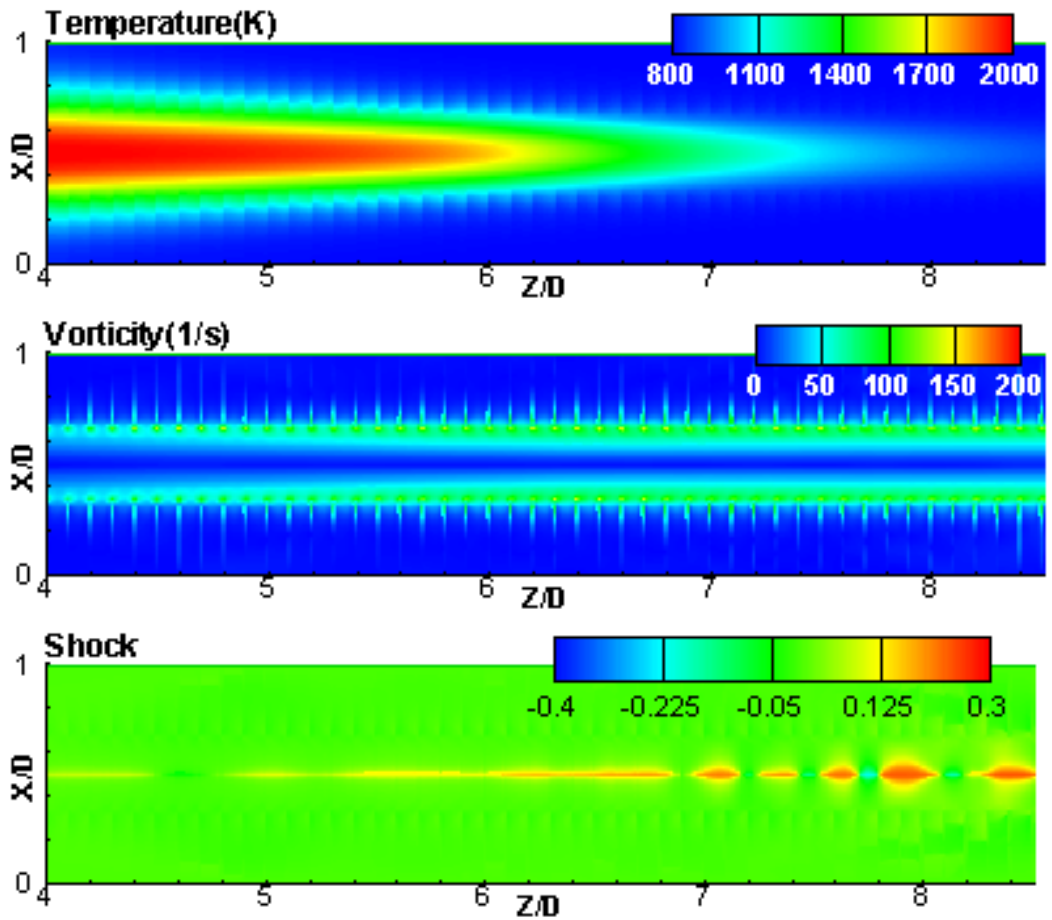

Figure 9.14: Flame snapshots for $\beta=80 \%, \alpha=2 / 3, D=12 \mu \mathrm{m}$. 
The flame shape does not show the appearance of small-scale wrinkling observed with lower dilution rates. Likewise, the increase in the blockage ratio also appears to increase the flame velocity. It is observed in the snapshot of the vorticity in Figs. $9.13-9.14$, that a shear layer develops from the edge of the obstacles which grows further downstream and appears stronger with blockage ratio $\alpha$. It is worth noting, that adding closely packed obstacles into flow direction can help to provide acceleration of the front for the highly diluted flame. Figure 9.15 indicates the quasi-steady oscillations in $D=6 \mu \mathrm{m}$ channel caused by constriction of the thickened flame when the channel width $D \sim$ flame thickness $L_{f}$. The oscillation and the acceleration scenarios in highly diluted cases are presented using the scaled flame length $D_{F} / D$ similar to previous work [64]. It has been reported in previous studies of flame in channel that scaled velocity of the weakly corrugated flame correlates quite well with the scaled flame area. As the channel width increases beyond a certain threshold, when width $D \gg L_{f}$, the flame will accelerate, Fig. 9.16 shows this trend as it depends on the blockage ratio.

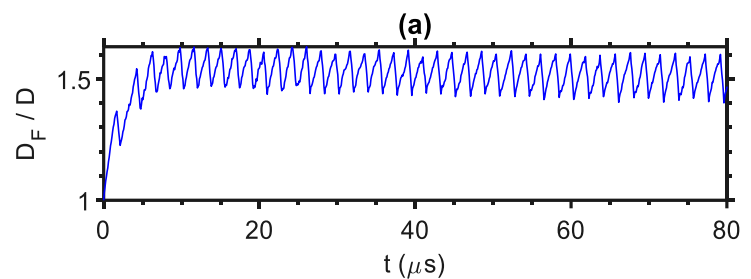

(b)

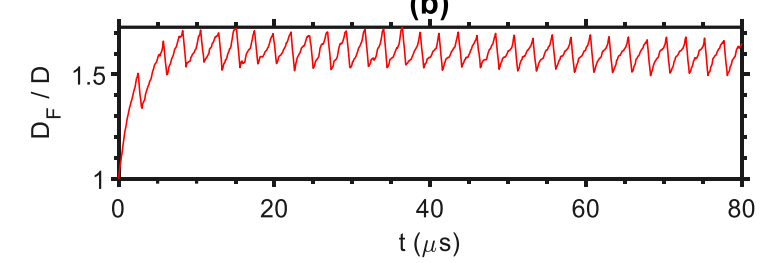

Figure 9.15: Scaled flame length vs time for $D=6 \mu \mathrm{m}$ and $\beta=80 \%$ a) $\alpha=1 / 2$, b) $\alpha=2 / 3$.

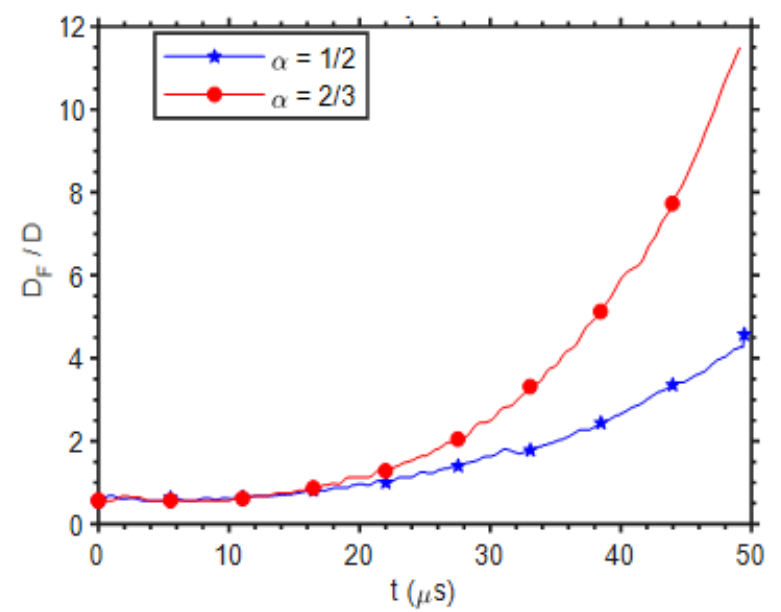

Figure 9.16: Scaled flame tip length vs time for $D=24 \mu m, \alpha=2 / 3$ and various $\beta$. 


\subsection{Comparison with Bychkov Theory}

Figure 9.17a shows the plot of the flame tip velocity normalized with the planar flame speeds of various $\mathrm{CO}_{2}$ dilution rate $\beta$ against a normalized time in the channel width $D=24 \mu \mathrm{m}$. The dashed line shows the Bychkov theory, Eqs. (2.1) - (2.2). These confirm that simulation reproduces the exponential acceleration trend with slight deviation from the theory. As seen, the scaled flame tip velocity profiles show an exponential acceleration trend and this acceleration rate decreases with the dilution rate $\beta$ as seen in Fig. 9.17b. The effects of high $\mathrm{CO}_{2}$ dilution on the acceleration rates are moderated in the Bychkov mechanism/framework significantly.
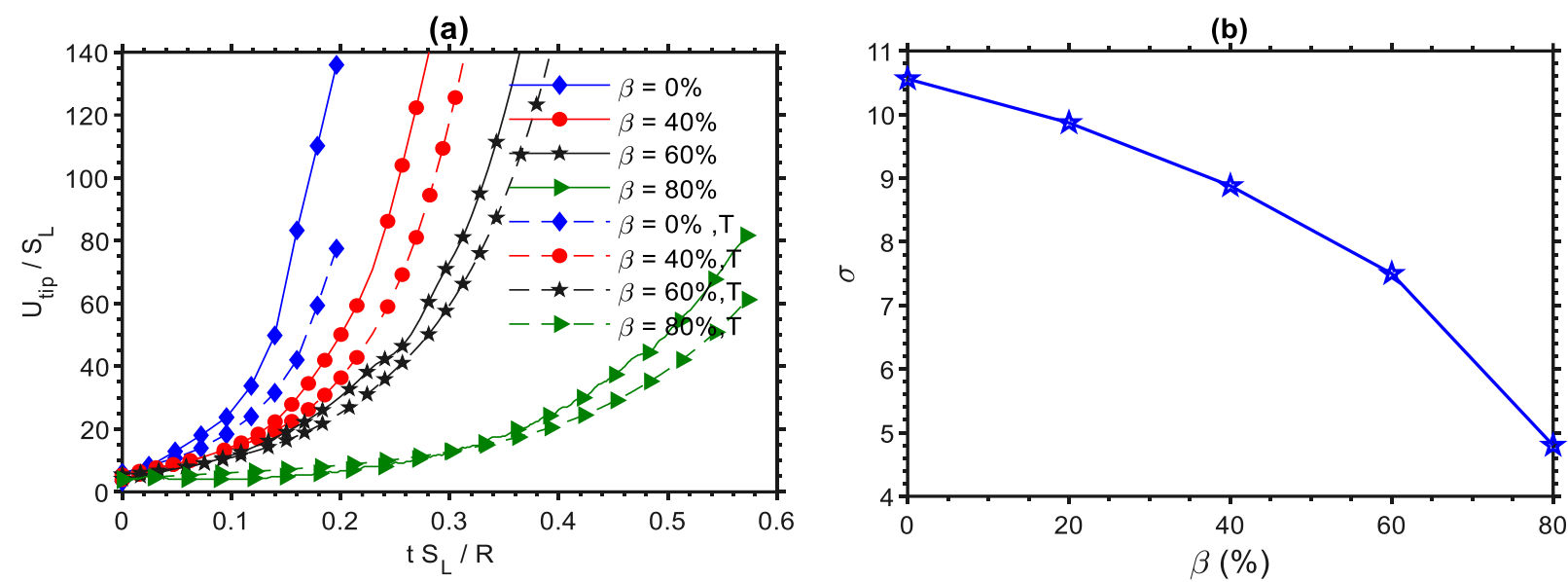

Figure 9.17: a) The scaled flame tip velocity vs time for $D=24 \mu m, \alpha=2 / 3$ b) scaled acceleration rates vs $\beta$.

The results of this comparison are shown in Figure 9.18. The acceleration trends closely match the predictions of Bychkov for weak to moderate dilution rates. Since these theories assumed gas incompressibility and infinitely thin flame. The model departs in the pure oxy-methane cases which are dominated by high compressibility and quick transitioning to saturated velocity driven by gas compressibility. At this rate, the theory breaks down, these are indicated by the deviation of the theory line. For the dilution rates of $\beta=40 \%, 60 \%$ the simulations match with the theory quite well. At dilution rates $\beta=80 \%$ the flame thickness grows significantly, perhaps these thickening causes the deviation of the simulation result from the theory. 
(a)

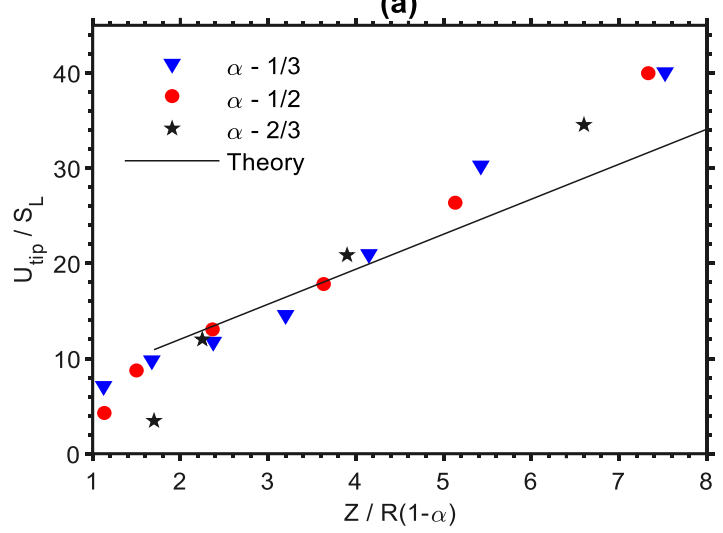

(c)

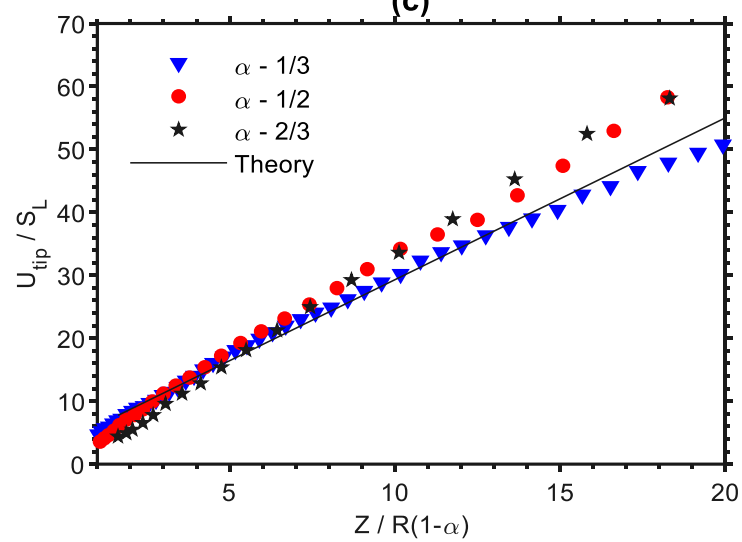

(b)

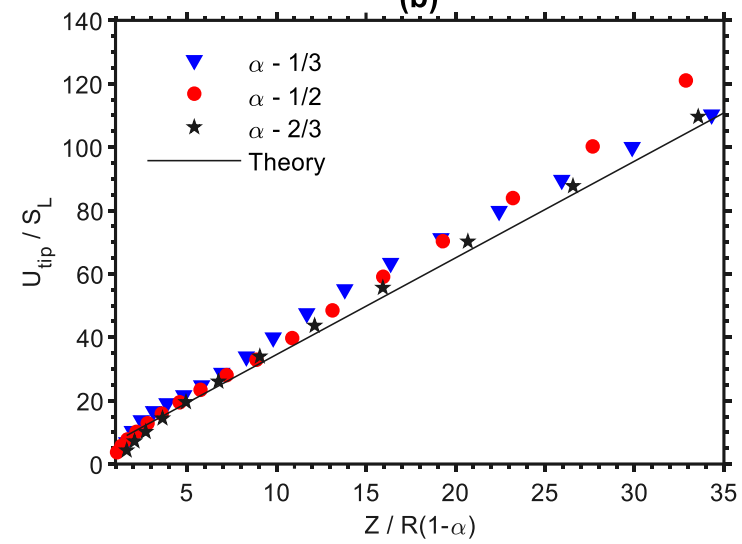

(d)

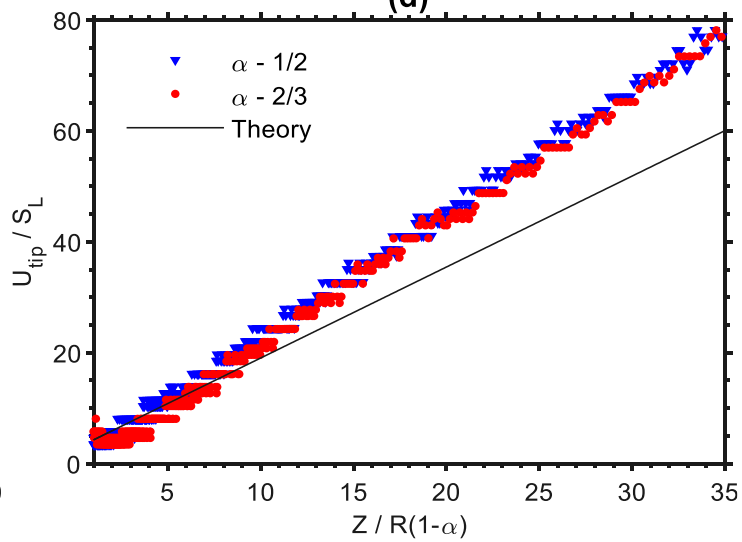

Figure 9.18: The scaled flame tip velocity versus the scaled flame tip position for $D=24 \mu \mathrm{m}$ for various $\beta$ 


\section{Conclusions and Recommendations}

\subsection{Conclusions}

This dissertation focusses on the nature and trends of premixed flames under atmospheric and supercritical conditions in both obstructed and unobstructed channels. Starting with flame propagation in obstructed cylindrical channels at atmospheric conditions. It is observed for small and moderate obstacle spacing, that an impact of wall shear stress on flame acceleration is minor, $1 \sim 4 \%$, slightly facilitating acceleration in a channel with $\Delta Z / R=1 / 2$ and moderating acceleration in the case of $\Delta Z / R=1 / 4$. However, the situation is different in the case of a wide spacing. Earlier, a minor effect of the isothermal surfaces as compared to the adiabatic ones was also demonstrated [18]. With the fact that the Bychkov model employed slip walls whereas the physical boundary conditions are non-slip as far as the continuum assumption is valid, the present work thereby justifies the Bychkov approach and makes it widely applicable to the practical reality; but within its validity limit. Indeed, at a large spacing, $\Delta Z \gg R$, the Bychkov model is not applicable anyway, and vorticity comes to play and show different behaviors for slip and nonslip walls. While the outcome of this work could be anticipated and explained by the fact that the flame dynamics is mainly driven by the flame spreading in the unobstructed portion of an obstructed channel (i.e. far from the wall), the situation is, however, qualitatively different from that in the unobstructed channels, where the wall shear stress and thermal wall conditions modify the flame dynamics.

A thorough investigation of FA in obstructed channels has been performed for nonequidiffusive fuel mixtures, by means of computational simulations, and a profound impact of Le on FA has been identified. This effect is compared to that of $\alpha$ and it has been found to be as strong. In the case of $L e<1$, promotion of flame acceleration is discovered. It is found that $L e$ has an influence on $\alpha$-dependence. In contrast, moderation of FA has been observed for $L e>1$ flames. In addition, a unique trend is noticed for the Le-impact on Re. Indeed, the Lewis number may change a $R e$-dependence of FA to an opposite trend. Also, flame oscillations are identified and scrutinized the in 2D obstructed channels with both ends open by means of the computational simulations. Specifically, the oscillations in the channels of half-width $R / L_{f}=R e=12$, the blockages ratios $\alpha=1 / 3,1 / 2,2 / 3$ and the obstacles spacing $\Delta Z / R=1 / 4,1 / 2,1$ were considered. It has been observed that $\Theta$ and $\alpha$ influence the oscillations both qualitatively and 
quantitatively. Overall, the oscillations differ conceptually from ultrafast flame acceleration in semi-open obstructed channels $[15,16]$, but they resemble the oscillations found in unobstructed channels with both ends open [4]. Moreover, treated as fluctuations around steady flame propagation, the oscillations agree with the modelling [35,36], experiments [34], and theory [36] of flames in channels with both ends open or vented in terms that all of the studies [35-37] identified steady flame propagation prior to fast acceleration.

While the observed oscillations were obtained in a relatively narrow channel, $R e=12$, at the channels of half-width $R / L_{f}=R e=24,36,48$, blockages ratios $\alpha=1 / 3,1 / 2,2 / 3$ and the thermal expansion ratios $\Theta=5,6,8,10$, and for obstacle spacing $\Delta \mathrm{Z}=R / 4$, the propagation trends observed are quite distinct. It consists of three phases, when the parameters $R e, \alpha$ and $\Theta$ are beyond a critical value. The phases are initial quasi-steady propagation, supplanted by an exponential acceleration and a final saturation of the flame velocity. Typically, the three phase regimes occur when $\Theta=8,10, \alpha=1 / 2,2 / 3$ and $R e=24,36,48$. It was noticed that all three parameters play a significant role in the duration of the quasi-steady propagation phase. Specifically, the quasi-steady propagation lasts for a scaled time of 6 with $R e=24$, and reduces to 0.6 when $R e=48$.

The regime of the quasi-steady propagation is also influenced by the blockage ratio $\alpha$ and the thermal expansion ratio $\Theta$. As the blockage ratio decreases, the volume of the pockets of unburned gases left behind by the leading flame front also reduces, causing drastic moderation of the delayed burning effect which is a major factor for flame propagation in the Bychkov mechanism. Likewise, the thermal expansion $\Theta$ plays a similar role, lower gas expansion, means reduced propulsion forces and as a result, reduced propagation of the flame front. The nature of the exponential acceleration was explored by comparing the simulation results with the theory developed by Bychkov for channels [36] with closely packed obstacles and both ends open. The plots of this comparison show that the theory predicts the later stage of the exponential acceleration quite better than it predicts the early phase.

The simulation results help to understand the regime of application of this theory to realistic flame propagation and identified the region to be somewhere between $1 / 3,6$ and $1 / 2,8$ for blockage ratio, thermal expansion ratio. Furthermore, the regime of the saturated velocity was investigated. The flame becomes saturated at close to Mach number of 1 . It was noticed that the 
main parameter that influences the final saturated velocity is the blockage ratio. Particularly, with $\alpha=1 / 2$, the flame saturated to a higher velocity than $\alpha=2 / 3$. The series of saturated velocities observed were also lower than the Chapman-Jouget deflagration velocity. Finally, a logistic regression machine learning algorithm was utilized to learn about the hypothesis functions that predict when a flame undergoes acceleration and when it continuously propagates in a quasi-steady state without acceleration. As such, the models which identify when the flame would experience at three regimes (quasi-steady, acceleration, saturation) and when it only propagates with nearconstant velocity, based on the Reynolds number, blockage ratio and thermal expansion ratio are developed.

The modeling of supercritical premixed stoichiometric oxy-methane burning in channels was numerically studied using an in-house compressible reacting flow solver extended for real gas flows using the Peng-Robinson equation of state and the Lucas viscosity model with one step chemistry. The oxy-methane flames are studied at elevated temperature and pressure of $800 \mathrm{~K}$ and 300 bar with $\mathrm{CO}_{2}$ diluents mainly to ensure that all participating species are at supercritical states and to mimic the high temperature levels due to exhaust gas recirculation. The viscosity of the supercritical oxy-methane mixture observed, shows a near linear dependence with pressure. However, at fixed temperature/pressure of $800 \mathrm{~K}$ and $300 \mathrm{bar}$, dilution with $\mathrm{CO}_{2}$ causes the viscosity to increase nonlinearly. The planar flame speed corresponds to $5.4 \mathrm{~m} / \mathrm{s}$ for pure oxymethane flame and decreases to $0.14 \mathrm{~m} / \mathrm{s}$ when diluted with 8 mols of $\mathrm{CO}_{2}$. This causes flame thickness to increase from $0.21 \mu \mathrm{m}$ to $3.53 \mu \mathrm{m}$. These data sets would be invaluable to design of future gas turbine combustors.

The extension of analysis to that of corrugated flame due to the influence of wall boundaries show that the flame shapes for the pure and low $\mathrm{CO}_{2}$ diluted conditions are concave with respect to the unburnt gases and become convex when highly diluted with $\mathrm{CO}_{2}$. The concave flame evolves from a three-part form into a "tulip" one with the tip pointing towards the burnt gases. The corrugated flame propagates exponentially at the onset then transitions into a quasi-linear propagation. It was also observed that the regime of the exponential propagation corresponds to the phase of the three-part flame and changes to quasi-linear trend when the flame shape form switches to the tulip. Overall, the corrugated velocity is largely subsonic, and the flame appears to exhibit some levels of instability when the channel widths increases. Furthermore, the wall shear 
stress and the shear layer ahead of the flame were explored. A shear thinning was observed in the unburned fuel mixture ahead of the flame which thickens with $\mathrm{CO}_{2}$ dilution, moreover the wall shear stress decreases with dilutions.

To understand how the heat release affects the flame surface curvature and the corrugated flame speed, the Markstein length and Markstein number were estimated from spherically propagating flames simulations. The trends of the Markstein length with $\mathrm{CO}_{2}$ mimics the flame thickness quite well. Consequently, the Markstein number decreases linearly with $\mathrm{CO}_{2}$ dilutions. The concave flames i.e. flame with zero to low $\mathrm{CO}_{2}$ dilutions have positive Markstein length while the convex flame that corresponds to 8 mols of $\mathrm{CO}_{2}$ has a negative Markstein length. Since Markstein length is known mostly to be positive [65], these results show that the highly diluted flames impede the heat diffusion rates, leading to a decline in propagation rates. While these present computational simulations are limited to micro-scales, the size of realistic combustors would be several orders of magnitude greater. However, the results obtained show good insights into the nature and trends of supercritical oxy-methane flames with $\mathrm{CO}_{2}$ and its direct application could be achieved in micro-combustors.

The simulation of supercritical flames in obstructed channels produced great insights into the propagation of the flames and their morphology in channels with different blockage ratios and the $\mathrm{CO}_{2}$ dilutions. In oxy-methane flames at supercritical states $\left(T_{f}=800 \mathrm{~K}, P_{f}=300 \mathrm{bar}\right)$, the flows are dominated by high compressibility, leading shock wave and high magnitude vortices ahead of the flames. As a result, the flame rapidly accelerates, and transitions into a choked flame that is independent of the tube width. These propagation trends are solely devoted to the delayed burning in the pockets of unburnt fuel mixture left behind by the flame front, eventually creating a jet flow in the core of the channel (unobstructed portion), thereby generating a positive feedback between the jet and the flow. Well, this is the fundamental phenomenon behind the Bychkov mechanism which is also replicated in the supercritical methane flames. However, these simulation results reveal the extra effect of supercritical states in the Bychkov mechanism. At high blockage ratio $\alpha=2 / 3$ in the undiluted cases, the leading shock waves, apparent in the low blockage ratio cases appear damped. The shock is confined to flame and burnt gases. Similarly, the vorticity contour shows no presence of the leading vortices ahead of the flame front. Since these scenarios occur at saturated flame speeds (choked flame), it is possible that the flame front and the leading 
shock interact and coalesce into a single wave, this exists typically prior to transition to detonation of a flame.

The flame shape is concave and changes to convex forms when dilution rates increase. Also, with low dilution rate, $\beta=40 \%$, the trends are like $\beta=0$. The temperature contour show that the flame breaks up at the saturated speed regime for $\alpha=2 / 3$, which grows into the unburnt fuel and a new front is formed. At moderate dilution $\beta=40 \%$, the flame shapes are convex in form, with weak small-scale wrinkling but quite stable. Here, the compression wave travels ahead of the flame in all blockage ratios $\alpha$ considered and the strength of the leading wave increases with $\alpha$. Also, the flame propagation rates are independent of the width of the channel. For the high dilution rate $\beta=$ $80 \%$, it was noticed that the channel width needs to be sufficiently larger than the flame thickness to overcome the hydraulic resistance in the flow. As such, the front oscillates about a quasi-steady speed in the narrow channel and shows significant acceleration with widening of the channel. These simulations show that flame accelerates exponentially for all dilution rates and adding of obstacles causes significant acceleration in the highly diluted flames. The results also match the Bychkov theory quite well especially within the validity of this theory.

\subsection{Recommendations}

This dissertation focuses on extensive computational simulations of premixed flames in different channel geometries at atmospheric and supercritical conditions. However, there are still numerous areas of this problem yet unresolved.

The limit of the effect of the flame propagation Reynolds number on non-equidiffusive flames in obstructed channels demands further investigation. The assumption of $R e$-independence slightly collapses for $L e \gg 1$ and $L e \ll 1$, as such it is crucial to determine the bounding limits for the non-unity Le, when the Reynolds number independence assumption holds or collapse. Moreover, it is important to modify the theory of Bychkov [15] to extend it to various Le flames. It is also crucial to conduct non-unity Le, DDT studies, to understand how the thermal-diffusive instabilities influence flame transition to detonation.

In the investigation of premixed flames in a channel with both ends open, the focus was placed on the $L e=1$ flames. This also needs to be generalized to all classes of flames, it is important to study how $L e \neq 1$ flames propagate, to fully characterize the propagation of premixed 
flames in obstructed channels with both ends opened. Moreover, the effects of thermal boundary conditions on the oscillating flames observed need to be scrutinized, considering both cold and preheated walls. Additionally, the flame classification diagram needs to be improved using more data sets, to clearly classify the accelerating and non-accelerating regimes in obstructed channels with both ends opened. Also, the Bychkov theory for premixed flames in open channels [36] needs to be updated to incorporate the effect of gas compressibility.

This work ventured into the realm of supercritical flames propagation and provided some insights into the physics of premixed flames in smooth and obstructed channels. However, a lot is still unclear in the modeling of fuel at supercritical conditions. The absence of reliable chemical kinetics data at a high pressure and temperature still raises a lot of unanswered questions. Will the chemical kinetics at supercritical conditions greatly influence the propagation and the morphology of this flame significantly? Will $\mathrm{CO}_{2}$ at such condition decompose and participate in the reaction rather than act as a diluent as it was assumed in this study? This is the question that would be answered in future work. Finally, the platform developed for studying supercritical flames needs to be validated with experimental data as soon as they are available. 


\section{Appendices}

Appendix A: Fortran 77 Code of Peng Robinson Implementation

C Peng Robinson EoS

$$
\begin{aligned}
& \mathrm{UG}=8.31434 \mathrm{D} 0 \\
& \mathrm{WM}=0.026667 \mathrm{D} \theta \\
& \mathrm{XDIL}=0.0 \mathrm{D} 0 \\
& \mathrm{XCH} 4=0.3333 \mathrm{D} 0 \\
& \mathrm{XO2}=0.6667 \mathrm{D} 0 \\
& \mathrm{XCO2}=0.3333 \mathrm{D} 0 \\
& \mathrm{XH} 20=0.6667 \mathrm{D} 0 \\
& \mathrm{XR}=\mathrm{YR} \\
& \mathrm{XB}=\mathrm{YB} \\
& \mathrm{VM}=\mathrm{WM} / \mathrm{RO}(\mathrm{K})
\end{aligned}
$$

C $\mathrm{CH} 4$

$\mathrm{TCCH} 4=190.8 \mathrm{D} 0$

$\mathrm{PcCH} 4=46.4 \mathrm{D} 5$

$\mathrm{VCCH} 4=9.93 \mathrm{D}-5$

$\mathrm{OMCH} 4=0.01 \mathrm{D} 0$

$\mathrm{BPCH} 4=0.016 \mathrm{D} 0$

$\mathrm{DcCH} 4=161.128$

$\mathrm{ZcCH} 4=0.740$

C $\quad 02$

$$
\begin{aligned}
\mathrm{TCO} 2 & =154.8 \mathrm{D} 0 \\
\mathrm{PCO} 2 & =50.8 \mathrm{D} 5 \\
\mathrm{~V} O 2 & =7.8 \mathrm{D}-5 \\
\mathrm{OMO} 2 & =0.022 \mathrm{D} 0 \\
\mathrm{BPO} 2 & =0.032 \mathrm{D} 0 \\
\mathrm{DCO} 2 & =410.26 \\
\mathrm{ZcO} 2 & =0.302
\end{aligned}
$$

C $\mathrm{CO} 2$

$\mathrm{TCCO} 2=304.2 \mathrm{DO}$

$\mathrm{PcCO} 2=73.9 \mathrm{D} 5$

$\mathrm{VCCO} 2=9.43 \mathrm{D}-5$

OMCO2 $=0.228 \mathrm{DO}$

$\mathrm{BPCO2}=0.044 \mathrm{DO}$

$\mathrm{DcC02}=466.596$

$\mathrm{ZcCO} 2=0.275$

C $\mathrm{H} 2 \mathrm{O}$

$\mathrm{TcH} 2 \mathrm{O}=647.1 \mathrm{DO}$

$\mathrm{PcH} 2 \mathrm{O}=220.6 \mathrm{D} 5$

$\mathrm{VcH} 2 \mathrm{O}=5.6 \mathrm{D}-5$ 


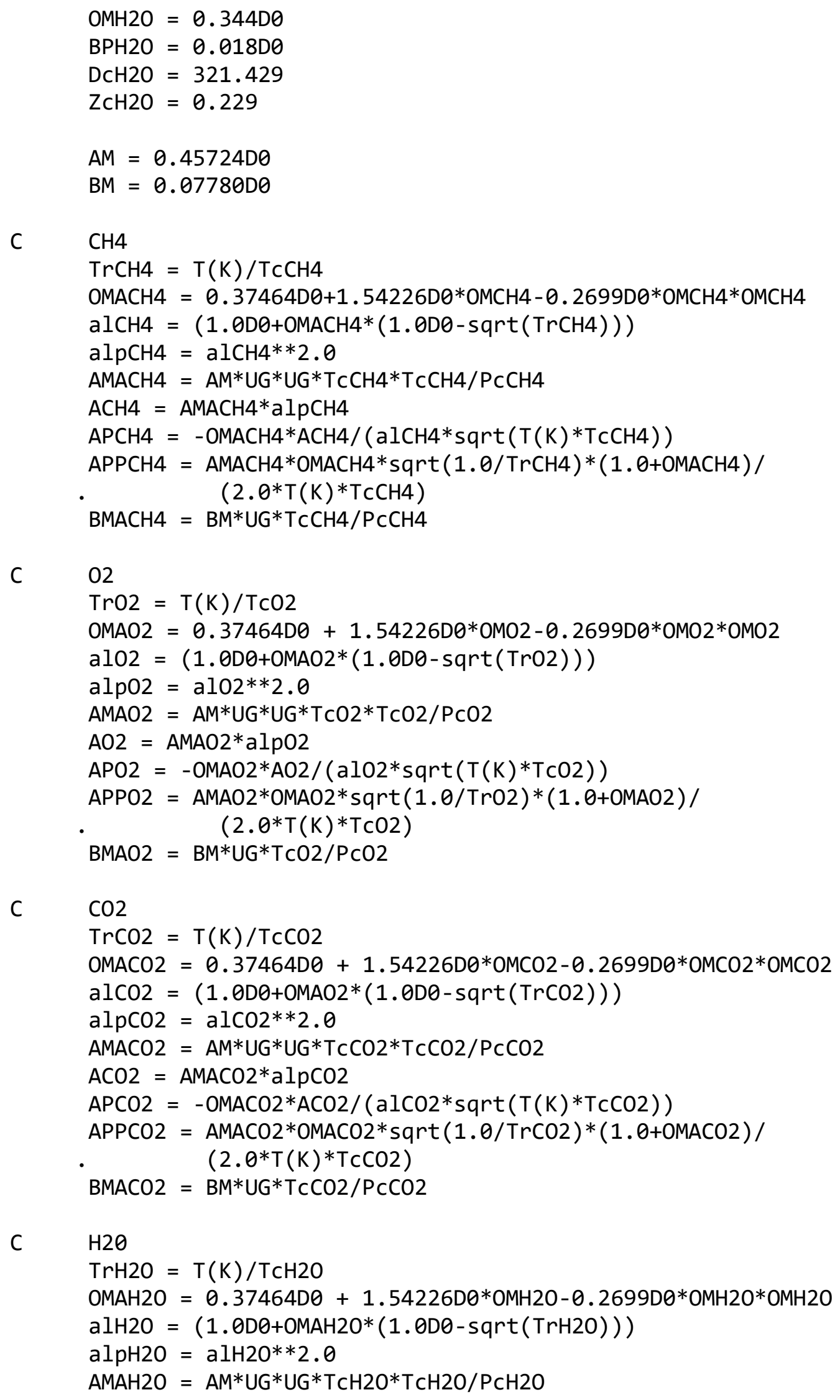




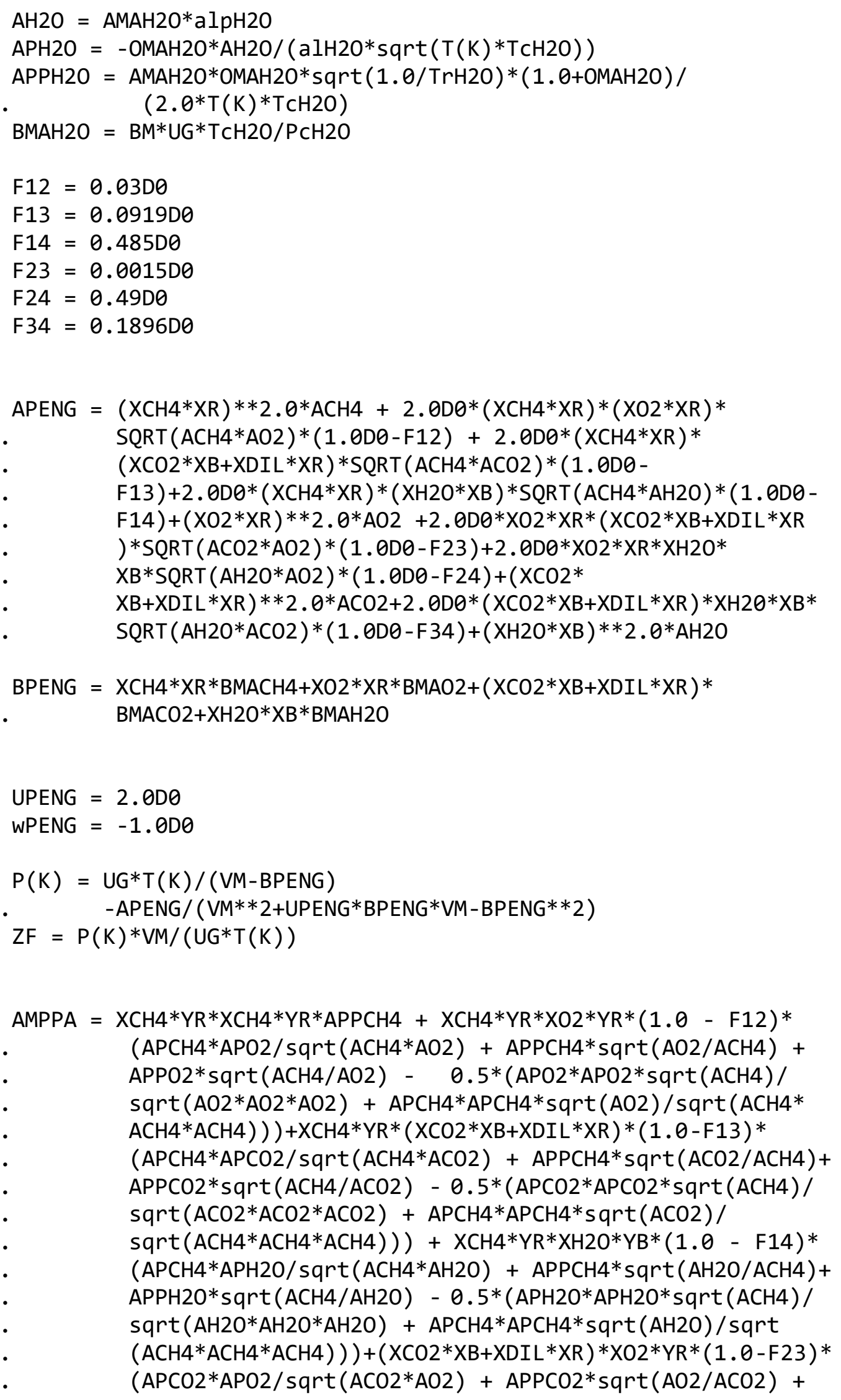




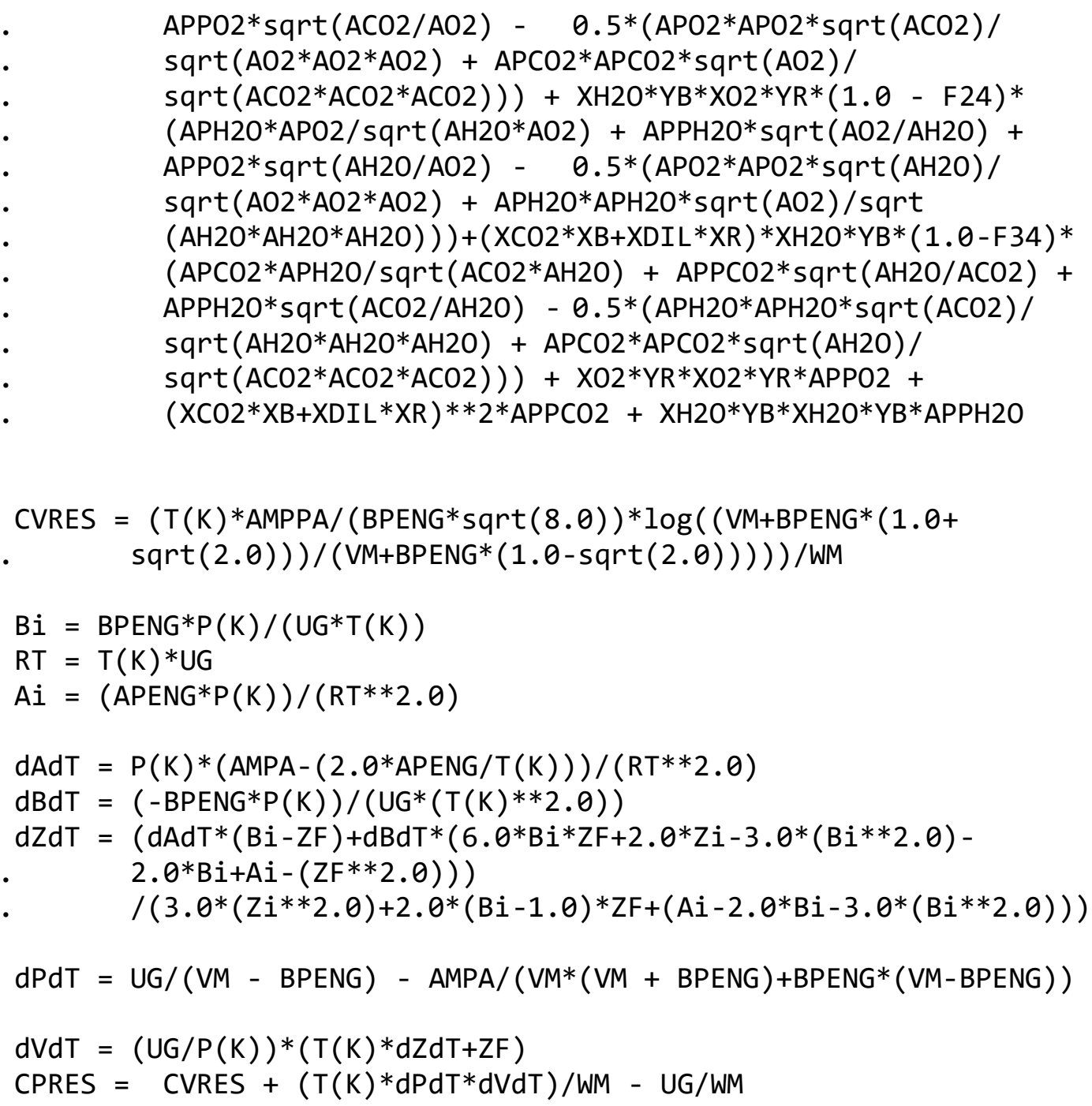

Appendix B: Fortran 77 Code of Lucas Viscosity Model

C Lucas et al. Method for High Pressure Viscosity Calculation

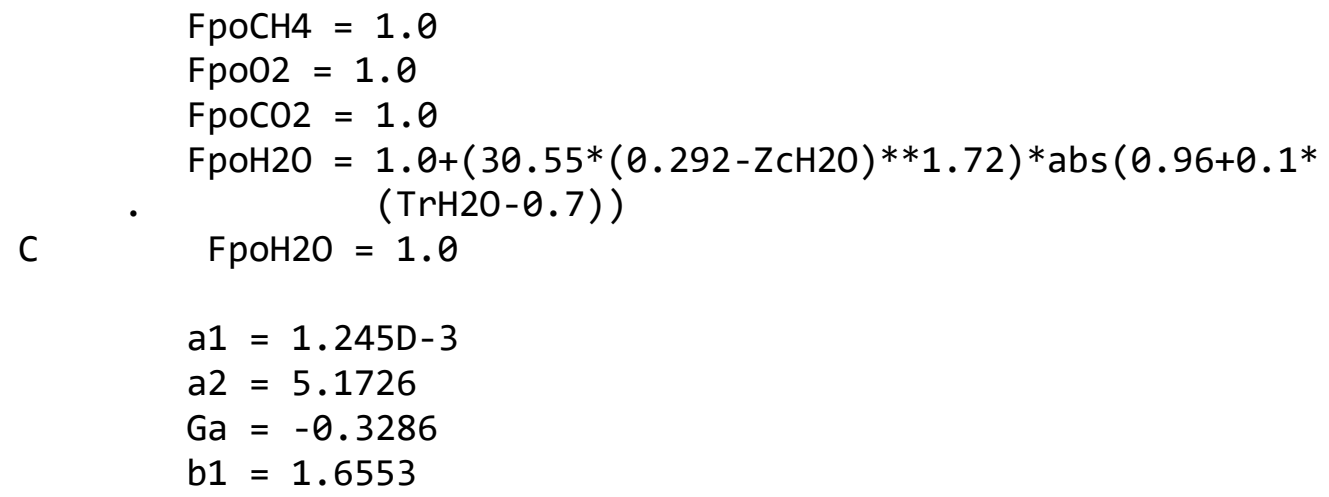




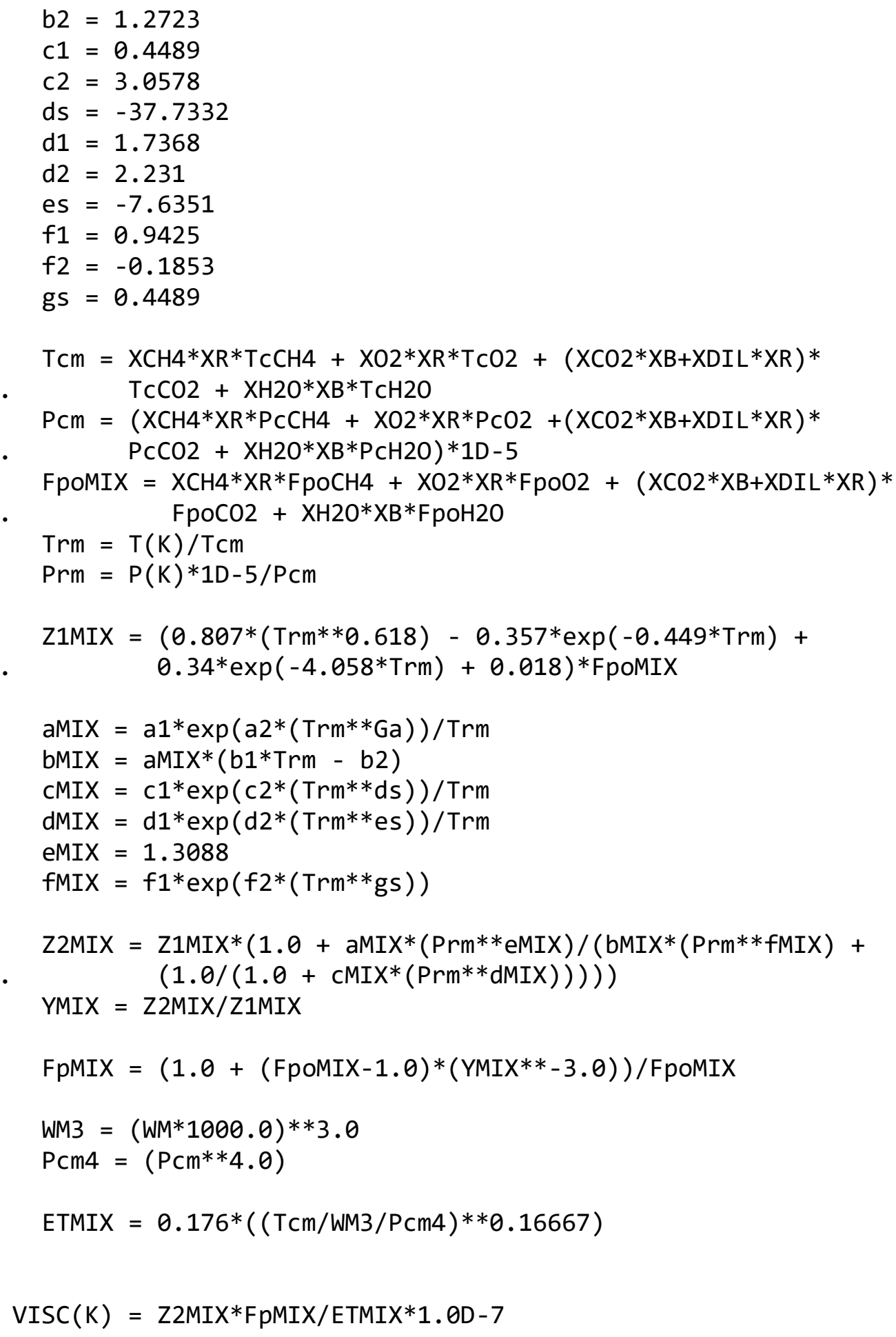


Appendix C: C Code of Peng Robinson Implementation

//Peng Robinson Def

//double $Y B=1.0$ - dat->Y[j][i];

$V M=W M /$ dat $->\operatorname{Ro}[j][i] ;$

// $\mathrm{CH} 4$

$\mathrm{TrCH} 4=$ dat $->\mathrm{T}[j][\mathrm{i}] / \mathrm{TcCH} 4$;

OMACH4 $=0.37464+1.54226 * 0 M C H 4-0.2699 * 0 M C H 4 * 0 M C H 4$;

alCH4 = (1.0+OMACH4*(1.0-sqrt $(\operatorname{TrCH} 4)))$;

$\operatorname{alpCH} 4=\operatorname{pow}(\mathrm{alCH} 4,2.0)$;

AMACH4 = AM*UG*UG*TcCH4*TcCH4/PcCH4;

$\mathrm{ACH} 4=\mathrm{AMACH} 4 * \mathrm{alpCH} 4$

$\mathrm{BMACH} 4=\mathrm{BM} * \mathrm{UG} * \mathrm{TCCH} 4 / \mathrm{PCCH} 4$;

/ $\quad 02$

$\operatorname{TrO2}=$ dat $->\mathrm{T}[\mathrm{j}][\mathrm{i}] / \mathrm{TcO} 2 ;$

OMAO2 $=0.37464+1.54226 * 0 M 02-0.2699 * 0 M 02 * 0 M 02$;

al02 $=(1.0+0 M A 02 *(1.0-\operatorname{sqrt}(\operatorname{TrO} 2)))$;

alp02 = pow $(a 102,2.0)$;

AMAO2 = AM*UG*UG*TCO2*TC02/Pc02;

$\mathrm{AO2}=\mathrm{AMAO2} * \mathrm{alp02}$

$\mathrm{BMAO2}=\mathrm{BM} * \mathrm{UG} * \mathrm{TCO} 2 / \mathrm{PcO} 2$;

// $\quad \mathrm{CO} 2$

$\operatorname{TrCO} 2=$ dat $->\mathrm{T}[\mathrm{j}][\mathrm{i}] / \mathrm{TcCO} 2 ;$

OMACO2 $=0.37464+1.54226 *$ OMCO2 $-0.2699 *$ OMCO2 $*$ OMCO2;

$\operatorname{alCO} 2=(1.0+0 M A 02 *(1.0-\operatorname{sqrt}(\operatorname{TrCO} 2)))$;

alpCO2 = pow $(\operatorname{alcO} 2,2.0)$;

AMACO2 = AM*UG*UG*TcCO2*TcCO2/PcCO2;

$\mathrm{ACO} 2=\mathrm{AMACO} 2 * \mathrm{alpCO} ;$

$\mathrm{BMACO} 2=\mathrm{BM} * \mathrm{UG} * \mathrm{TCCO} 2 / \mathrm{PCCO}$;

// $\mathrm{H} 20$

$\mathrm{TrH} 2 \mathrm{O}=$ dat $->\mathrm{T}[\mathrm{j}][\mathrm{i}] / \mathrm{TcH} 2 \mathrm{O}$;

OMAH2O $=0.37464+1.54226 *$ OMH2O-0.2699*OMH2O*OMH2O;

alH2O = (1.0+OMAH2O* (1.0-sqrt $(\operatorname{TrH} 20)))$;

$\mathrm{alpH} 20=\operatorname{pow}(\mathrm{alH} 2 \mathrm{O}, 2.0)$;

AMAH2O = AM*UG*UG*TcH2O*TcH2O/PcH2O;

$\mathrm{AH} 2 \mathrm{O}=\mathrm{AMAH} 2 \mathrm{O} * \mathrm{alpH} 2 \mathrm{O}$;

$\mathrm{BMAH} 2 \mathrm{O}=\mathrm{BM} * \mathrm{UG} * \mathrm{TCH} 2 \mathrm{O} / \mathrm{PCH} 2 \mathrm{O}$;

$\mathrm{F} 12=0.03$;

$\mathrm{F} 13=0.0919 ;$

$\mathrm{F} 14=0.485 ;$

$F 23=0.0015 ;$ 


$$
\begin{aligned}
& \mathrm{F} 24=0.49 ; \\
& \mathrm{F} 34=0.1896 ; \\
& \mathrm{XDIL}=0.5 ; \\
& \mathrm{XCH4}=0.1667 ; \\
& \mathrm{XO2}=0.3333 ; \\
& \mathrm{XCO2}=0.6667 ; \\
& \mathrm{XH} 20=0.3333 ;
\end{aligned}
$$

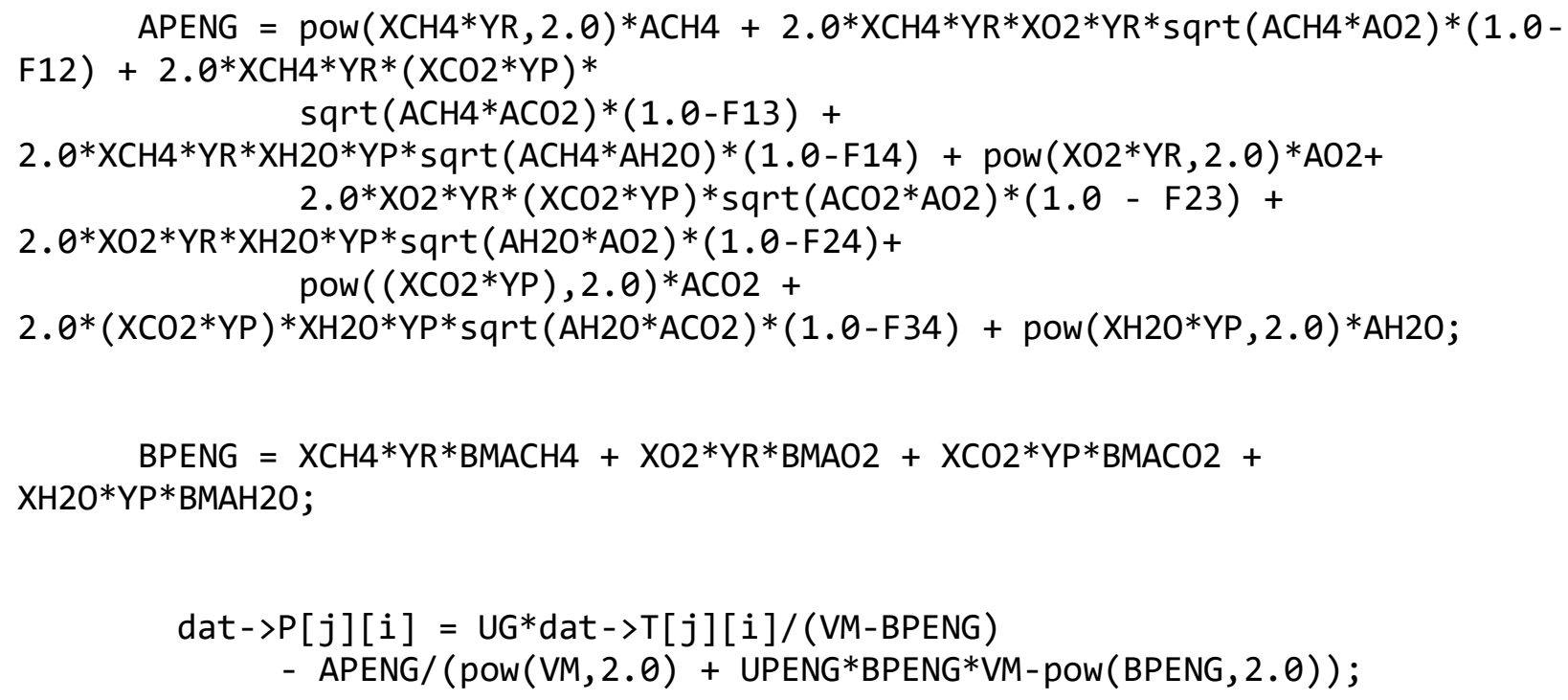

Appendix D: C Code of Lucas Viscosity Model

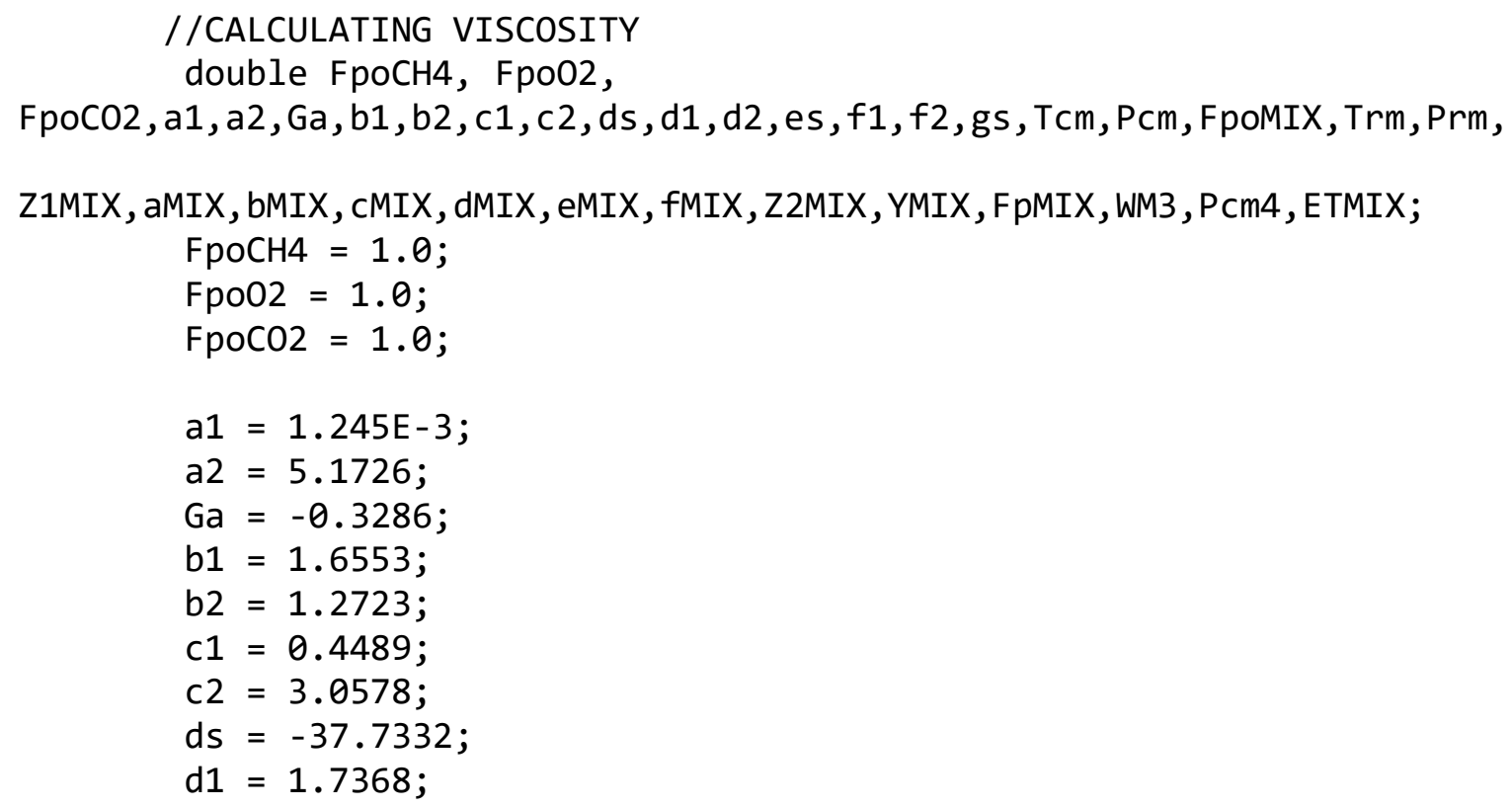




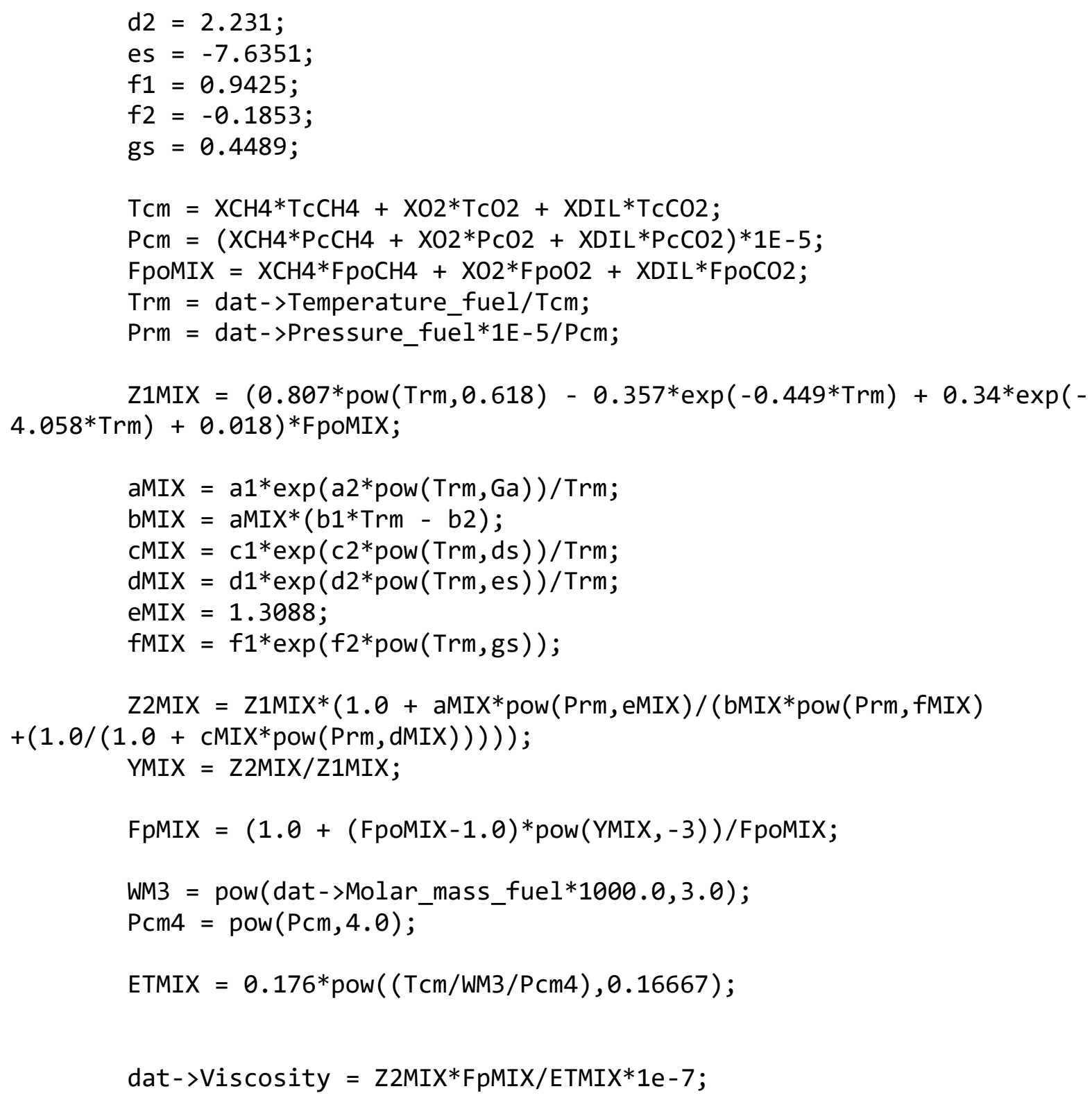




\section{References}

[1] U.S. Department of Energy, "Advancing Clean Electric Power Technologies Technology”, Quadrennial Technology Review (2015).

[2] H. Kobayashi, H. Hagiwara, H. Kaneko, and Y. Ogami, "Effects of $\mathrm{CO}_{2}$ dilution on turbulent premixed flames at high pressure and high temperature", Proceedings of the Combustion Institute, Vol. 31, pp. 1451-1458 (2007).

[3] Y. Ju and K. Maruta, "Microscale combustion: Technology development and fundamental research", Progress in Energy and Combustion Science, Vol. 37, No. 1, pp. 669-715 (2011).

[4] V. Akkerman, V. Bychkov, A. Petchenko, and L. E. Eriksson, "Flame oscillations in tubes with nonslip at the walls", Combustion and Flame, Vol. 145, No. 2, pp. 675-687 (2006).

[5] V. Bychkov, A. Petchenko, V. Akkerman, and L. E. Eriksson, "Theory and modeling of accelerating flames in tubes", Physical Review E, Vol. 72, p. 046307 (2005).

[6] V. Akkerman, C. K. Law, V. Bychkov, and L.-E. Eriksson, "Analysis of flame acceleration induced by wall friction in open tubes", Vol. 22, No. 5, p. 053606 (2010).

[7] K. Shelkin, "Influence of the wall roughness on initiation and propagation of detonation in gases", Journal of Experimental and Theoretical Physics, Vol. 10, pp. 823827 (1940).

[8] G. D. Roy, S. M. Frolov, A. A. Borisov, and D. W. Netzer, "Pulse detonation propulsion: Challenges, current status, and future perspective", Progress in Energy and Combustion Science, Vol. 30, No. 6, pp. 549-672 (2004).

[9] M. Lam, D. Tillie, T. Leaver, B. McFadden, "Pulse detonation engine technology: an overview", Applied Science 201, University of British Columbia (2004).

[10] V. N. Gamezo, T. Ogawa, and E. S. Oran, "Numerical simulations of flame propagation and DDT in obstructed channels filled with hydrogen-air mixture", Proceedings of the Combustion Institute, Vol. 31, No. 2, pp. 2463 - 2471 (2007).

[11] C. Wang, X. Dong, J. Cao, and J. Ning, "Experimental Investigation of Flame Acceleration and Deflagration-to-Detonation Transition Characteristics Using Coal Gas and Air Mixture", Combustion Science and Technology, Vol. 187, No. 11, pp. 1805 -1820 (2015).

[12] B. Zhang, H. Liu, and C. Wang, "On the detonation propagation behavior in hydrogenoxygen mixture under the effect of spiral obstacles", International Journal of Hydrogen Energy, Vol. 42, No. 33, pp. 21392 - 21402 (2017). 
[13] G. Ciccarellia and S. Dorofeev, "Flame acceleration and transition to detonation in ducts", Progress in Energy and Combustion Science, Vol. 34, No. 4, pp. 499 - 550 (2008).

[14] G. Ciccarelli, C. T. Johansen, and M. Parravani, "The role of shock-flame interactions on flame acceleration in an obstacle laden channel", Combustion and Flame, Vol. 157, No. 11, pp. $2125-2136$ (2010).

[15] V. Bychkov, D. Valiev, and L. E. Eriksson, "Physical mechanism of ultrafast flame acceleration", Physical Review Letters, Vol. 101, p. 164501 (2008).

[16] D. Valiev, V. Bychkov, V. Akkerman, C. K. Law, and L. E. Eriksson, "Flame acceleration in channels with obstacles in the deflagration-to-detonation transition", Combustion and Flame, Vol. 157, No. 5, pp. 1012 - 1021 (2010).

[17] V. Bychkov, V. Akkerman, D. Valiev, and C. K. Law, "Influence of gas compression on flame acceleration in channels with obstacles", Combustion and Flame, Vol. 157, No. 10, pp. $2008-2011$ (2010).

[18] O. J. Ugarte, V. Bychlov, J. Sadek, D. Valiev and V. Akkerman "Critical role of blockage ratio for flame acceleration in channels with tightly spaced obstacles", Physics of Fluids, Vol. 28, No. 9, p. 093602 (2016).

[19] A. Sahoo, "Flame Acceleration in an Obstacle-Laden Tube", Indian Institute of Technology, Madras (2017).

[20] C. T. Johansen and G. Ciccarelli, "Visualization of the unburned gas flow field ahead of an accelerating flame in an obstructed square channel", Combustion and Flame, Vol. 156, No. 2, pp. $405-416$ (2009).

[21] V. Akkerman and D. Valiev, "Moderation of flame acceleration in obstructed cylindrical pipes due to gas compression", Physics of Fluids, Vol. 30, p. 106101 (2018).

[22] M. Mizomoto and H. Yoshida, "Effects of Lewis number on the burning intensity of Bunsen flames", Combustion and Flame, Vol. 70, No. 1, pp. 47 - 60 (1987).

[23] A. McClung, J. Delimont, and S. Coogan, "High Inlet Temperature Combustor for Direct Fired Supercritical Oxy-Combustion", University Turbine Systems Research Workshop (2015).

[24] R. Wheeler and W. Mason, "The propagation of flame in mixtures of methane and air", Journal of the Chemical Society, Transactions, Vol. 117, pp. $36-47$ (1920).

[25] G. Ciccarelli, C. J. Fowler, and M. Bardon, "Effect of obstacle size and spacing on the initial stage of flame acceleration in a rough tube", Shock Waves, Vol. 14, No. 3, pp. 161-166 (2005).

[26] C. Johansen and G. Ciccarelli, "Numerical simulations of the flow field ahead of an accelerating flame in an obstructed channel", Combustion Theory and Modelling, Vol. 14, No. 2, pp. 235 - 255 (2010). 
[27] C. Wang, F. Huang, E. K. Addai, and X. Dong, "Effect of concentration and obstacles on flame velocity and overpressure of methane-air mixture", Journal of Loss Prevention in the Process Industries, Vol. 43, pp. $302-310$ (2016).

[28] C. Dong, M. Bi, and Y. Zhou, "Effects of obstacles and deposited coal dust on characteristics of premixed methane-air explosions in a long closed pipe", Safety Science, Vol. 50, No. 9, pp. 1786-1791 (2012).

[29] A. Heidari and J. X. Wen, "Numerical simulation of flame acceleration and deflagration to detonation transition in hydrogen-air mixture", International Journal of Hydrogen Energy, Vol. 39, No. 36, pp. 21317-21327 (2014).

[30] L. R. Boeck, S. Lapointe, J. Melguizo-Gavilanes, and G. Ciccarelli, "Flame propagation across an obstacle: OH-PLIF and 2-D simulations with detailed chemistry", Proceedings of the Combustion Institute, Vol. 36, No. 2, pp. 2799-2806 (2017).

[31] D. A. Kessler, V. N. Gamezo, and E. S. Oran, "Simulations of flame acceleration and deflagration-to-detonation transitions in methane-air systems", Combustion and Flame, Vol. 157, No. 11, pp. 2063-2077 (2010).

[32] L. R. Boeck, M. Kellenberger, G. Rainsford, and G. Ciccarelli, "Simultaneous OH-PLIF and schlieren imaging of flame acceleration in an obstacle-laden channel", Proceedings of the Combustion Institute, Vol. 36, No. 2, pp. 2807-2814 (2017).

[33] A. Teodorczyk, P. Drobniak, and A. Dabkowski, "Fast turbulent deflagration and DDT of hydrogen-air mixtures in small obstructed channel", International Journal of Hydrogen Energy, Vol. 34, No. 14, pp. 5887 - 5893 (2009).

[34] J. Yanez, M. Kuznetsov, and V. Bykov, "Sudden acceleration of flames in open channels driven by hydraulic resistance", Karlsruhe Institute of Technology (2013). http://arxiv.org/abs/1208.64

[35] P. Middha and O. Hansen, "Predicting Deflagration to Detonation Transition in Hydrogen Explosions", Process Safety Progress, Vol. 27, No. 3, pp. 192 - 204 (2008).

[36] V. Bychkov, V. Akkerman, and J. Sadek, "Analysis of flame acceleration in open or vented obstructed pipes”, Physical Review E, Vol. 95, p. 013111 (2017).

[37] X. Hu, Q. Yu, J. Liu, and N. Sun, "Investigation of laminar flame speeds of $\mathrm{CH}_{4} / \mathrm{O}_{2} / \mathrm{CO}_{2}$ mixtures at ordinary pressure and kinetic simulation", Energy, Vol. 70, pp. $626-634$, (2014).

[38] Z. Yang, X. Yu, J. Peng, L. Wang, Z. Dong, X. Li, S. Sun, S. Meng, H. Xu, "Effects of N2, $\mathrm{CO}_{2}$ and $\mathrm{H}_{2} \mathrm{O}$ dilutions on temperature and concentration fields of $\mathrm{OH}$ in methane Bunsen flames by using PLIF thermometry and bi-directional PLIF", Experimental Thermal and Fluid Science, Vol. 81, pp. 209-222 (2017).

[39] N. Ding, R. Arora, M. Norconk, and S. Y. Lee, "Numerical investigation of diluent influence on flame extinction limits and emission characteristic of lean-premixed $\mathbf{H}_{2}-$ 
CO (syngas) flame”, International Journal of Hydrogen Energy, Vol. 36, No. 4, pp. 32223231 (2011).

[40] R. Shang, Y. Zhang, M. Zhu, Z. Zhang, D. Zhang, and G. Li, "Laminar flame speed of $\mathrm{CO}_{2}$ and $\mathrm{N}_{2}$ diluted $\mathrm{H}_{2} / \mathrm{CO} /$ air flames", International Journal of Hydrogen Energy, Vol. 41, No. 33, pp. 15056-15067 (2016).

[41] M. Mendieta, D. Alviso, and R. Gonçalves dos Santos, "Numerical Study of Laminar Premixed Methane/Air Flames With Carbon Dioxide Dilution", $16^{\text {th }}$ Brazilian Congress of Thermal Sciences and Engineering, , Vitória, ES, Brazil, November 07 - 10 (2016).

[42] Y. L. Chan, M. M. Zhu, Z. Z. Zhang, P. F. Liu, and D. K. Zhang, "The Effect of CO2 Dilution on the Laminar Burning Velocity of Premixed Methane/Air Flames", Energy Procedia, Vol. 75, pp. 3048 - 3053 (2015).

[43] Y. Zhang, W. Shen, H. Zhang, Y. Wu, and J. Lu, "Effects of inert dilution on the propagation and extinction of lean premixed syngas/air flames”, Fuel, Vol. 157, pp. 115 $-121(2015)$.

[44] C. Zhang, G. Hu, S. Liao, Q. Cheng, C. Xiang, and C. Yuan, "Comparative study on the effects of nitrogen and carbon dioxide on methane/air flames", Energy, Vol. 106, pp. $431-442$ (2016).

[45] A. R. Khan, S. Anbusaravanan, L. Kalathi, R. Velamati, and C. Prathap, "Investigation of dilution effect with $\mathrm{N}_{2} / \mathrm{CO}_{2}$ on laminar burning velocity of premixed methane/oxygen mixtures using freely expanding spherical flames", Fuel, Vol. 196, pp. 225-232 (2017).

[46] H. Kobayashi, Y. Otawara, J. Wang, F. Matsuno, Y. Ogami, M. Okuyama, T, Kudo, S. Kadowaki, "Turbulent premixed flame characteristics of a $\mathrm{CO} / \mathrm{H}_{2} / \mathrm{O}_{2}$ mixture highly diluted with $\mathrm{CO}_{2}$ in a high-pressure environment", Proceedings of the Combustion Institute, Vol. 34, No. 1, pp. 1437 - 1445 (2013).

[47] P. Uddholm, "Numerical Simulation of Flame Propagation”, Uppsala Universitet (2008). http://uu.diva-portal.org/smash/get/diva2:174140/FULLTEXT01.pdf.

[48] L. Cutrone, M. Ihme, and M. Herrmann, "Modeling of high-pressure mixing and combustion in liquid rocket injectors", Center for Turbulence Research, Proceedings of the Summer Program (2006).

[49] S. K. Fateen, M. M. Khalil, and A. O. Elnabawy, "Semi-empirical correlation for binary interaction parameters of the Peng-Robinson equation of state with the van der Waals mixing rules for the prediction of high-pressure vapor-liquid equilibrium", Journal of Advanced Research, Vol. 4, No. 2, pp. 137 - 145 (2013).

[50] I. Soreide and C. Whitson, "Peng-Robinson predictions for hydrocarbons, $\mathrm{CO}_{2}, \mathbf{N}_{2}$, and $\mathrm{H}_{2} \mathrm{~S}$ with pure water and NaCI brine”, Fluid Phase Equilibria, Vol. 77, pp. 217-240 (1992).

[51] A. Burcat, "Combustion Chemistry", (1983). 
[52] V. Yang, "Modeling of supercritical vaporization, mixing, and combustion processes in liquid-fueled propulsion systems", Proceedings of the Combustion Institute, Vol. 28, No. 1, pp. 925-942 (2000).

[53] B. Poling, J. Prausnitz, and J. O'Connell, "The Properties of Gases and Gas Mixtures", (2000).

[54] Y. Zeldovich, R. Barenblatt, V. Librovich, and G. Makhviladze, "Mathematical Theory of Combustion and Explosions", 1985.

[55] V. V Bychkov, S. M. Golberg, M. A. Liberman, and L. E. Eriksson, "Propagation of curved stationary flames in tubes", Physical Review E, Vol. 54, No. 4, pp. 3713 - 3724 (1996).

[56] B. Demirgok, O. Ugarte, D. Damir, C.K. Law, M. Wu, V. Bychkov, V. Akkerman, "Analysis of Ethylene-Oxygen Combustion in Micro-Pipes", U.S. National Combustion Meeting (2015).

[57] O. J. Ugarte-Almeyda, "Effect of boundary conditions on propagation and morphology of premixed flames in narrow conduits effect of boundary conditions on propagation and morphology of premixed flames in narrow conduits", West Virginia University, (2015).

[58] “CHEMKIN-PRO 15131”, Reaction Design, San Diego (2013).

[59] J. Oh and D. Noh, "Laminar burning velocity of oxy-methane flames in atmospheric condition", Energy, Vol. 45, No. 1, pp. 669 - 675 (2012).

[60] Y. Lafay, B. Renou, G. Cabot, and M. Boukhalfa, "Experimental and numerical investigation of the effect of $\mathrm{H}_{2}$ enrichment on laminar methane-air flame thickness", Combustion and Flame, Vol. 153, No. 4, pp. 540 - 561 (2008).

[61] E. S. Oran, "A tribute to Dr. George H. Markstein (1911-2011)", Combustion and Flame, Vol. 1, No. 162, pp. 1 -2 (2015).

[62] P. Clavin, "Dynamic behavior of premixed flame fronts in laminar and turbulent flows", Progress in Energy and Combustion Science, Vol. 11, No. 1, pp. 1 - 59 (1985).

[63] Z. Chen, "On the extraction of laminar flame speed and Markstein length from outwardly propagating spherical flames", Combustion and Flame, Vol. 158, No. 2, pp. $291-300$ (2011).

[64] A. Adebiyi, E. Ridgeway, R. Alkandari, A. Cathreno, D. Valiev, and V. Akkerman, "Premixed flame oscillations in obstructed channels with both ends open", Proceedings of the Combustion Institute, Vol. 37, No. 2, pp. 1919-1926 (2019).

[65] G. Searby and J. Quinard, "Direct and indirect measurements of Markstein numbers of premixed flames", Combustion and Flame, Vol. 82, No. 3-4, pp. 298-311 (1990). 